\title{
Activity of M dwarfs in the CARMENES sample
}

\author{
Dissertation \\ zur Erlangung des mathematisch-naturwissenschaftlichen Doktorgrades \\ "Doctor rerum naturalium" \\ der Georg-August-Universität Göttingen \\ im Promotionsprogramm PROPHYS \\ der Georg-August University School of Science (GAUSS)
}

\author{
vorgelegt von \\ Patrick Schöfer \\ aus Northeim
}

Göttingen, 2021 
Betreuungsausschuss

Prof. Dr. Ansgar Reiners, Institut für Astrophysik, Georg-August-Universität Göttingen

Prof. Dr. Stefan Dreizler, Institut für Astrophysik, Georg-August-Universität Göttingen

Dr. Sandra Jeffers, Institut für Astrophysik, Georg-August-Universität Göttingen, bis 15 . November 2020

Mitglieder der Prüfungskommission

Referent: Prof. Dr. Ansgar Reiners

Institut für Astrophysik, Georg-August-Universität Göttingen

Korreferent: Prof. Dr. Stefan Dreizler

Institut für Astrophysik, Georg-August-Universität Göttingen

Weitere Mitglieder der Prüfungskommission:

Prof. Dr. David J. E. Marsh

Institut für Astrophysik, Georg-August-Universität Göttingen

Prof. Dr. Hardi Peter

Abteilung Sonne und Heliosphäre, Max-Planck-Institut für Sonnensystemforschung

Prof. Dr. Ariane Frey

II. Physikalisches Institut, Georg-August-Universität Göttingen

Prof. Dr. Andreas Tilgner

Institut für Geophysik, Georg-August-Universität Göttingen

Tag der mündlichen Prüfung: 18. Februar 2021 


\section{Die Pferde der Hoffnung galoppieren, doch die Esel der Erfahrung schreiten langsam. \\ — russisches Sprichwort}

The horses of hope gallop, but the asses of experience go slowly.

— Russian proverb 



\section{Abstract}

The Calar Alto high-Resolution search for M dwarfs with Exo-earths with Near-infrared and optical Echelle Spectrographs (CARMENES) has been searching and finding planets for five years by looking for periodic Doppler-shift variations down to $1 \mathrm{~m} \mathrm{~s}^{-1}$. One challenge in detecting planetary signals with this precision is that activity phenomena on the star can mimic Doppler signals in the same order of magnitude. While stellar activity imposes this challenge for finding planets, the spectra obtained in the survey also provide a treasury of data for studying stellar activity itself. In this thesis, I aim to quantify the stellar activity of $337 \mathrm{M}$ dwarfs in the CARMENES sample by measuring the strength of chromospheric emission lines and photospheric absorption bands, to identify correlations, and to investigate the temporal variability of the activity indicators. I used a spectral subtraction technique to measure pseudo-equivalent widths of the $\mathrm{He}_{\mathrm{I}} \mathrm{D}_{3}, \mathrm{H} \alpha$, He I $\lambda 10833 \AA$, and $\mathrm{Pa} \beta$ lines, the Na I D doublet, and the Ca II infrared triplet. For three TiO, two $\mathrm{CaH}$, and two VO absorption bands and the FeH Wing-Ford band, I calculated indices as the flux ratio of two spectral regions on either side of the band heads. Using generalised Lomb-Scargle periodograms, I searched for periodicities in the measured indicators. I analysed four out of eighteen stars that show a periodic modulation with the rotation period in more than two indicators in more detail.

I find that $\mathrm{He}$ I $\mathrm{D}_{3}$ and $\mathrm{H} \alpha$ show a very similar spectral-type dependence and are strongly correlated. For $\mathrm{Na}$ I $\mathrm{D}$ and the $\mathrm{Ca}$ II infrared triplet, the correlation with $\mathrm{H} \alpha$ is weaker, as the subtraction method cannot adjust for the very different shapes of these lines. No strong correlations with the other indicators are found for $\mathrm{He}$ I $\lambda 10833 \AA$ and $\mathrm{Pa} \beta$. The photospheric bands are strongly correlated with each other and show activity effects of different intensities. I outline a new indicator to quantify these effects. All results are in good agreement with previously published results. $\mathrm{He} \mathrm{I}_{3}$ and $\mathrm{H} \alpha$ show smaller relative variations at higher activity levels, whereas the relative variations of other indicators show no clear correlations. One $\mathrm{TiO}$ band, one $\mathrm{CaH}$ band, one line of the $\mathrm{Ca}$ II infrared triplet, and $\mathrm{H} \alpha$ undergo periodic modulations with the rotation period most commonly. The more detailed analysis of four stars shows the peculiarities in the signals of each star and reveals changing signals over time. 



\section{Contents}

1. Introduction 1

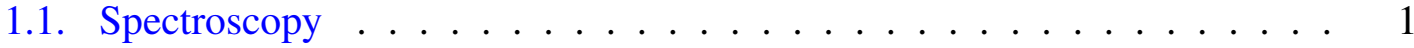

1.1.1. Spectral lines ................... 2

1.1.2. Spectrographs ................... 2

1.2. Star classification . . . . . . . . . . . . . . . 5 5

1.2.1. Spectral types .................. 5

1.2.2. Luminosity classes . . . . . . . . . . . . . . 5

1.2.3. Mdwarfs ...................... 6

1.2.4. Structure and atmospheres of main-sequence stars . . . . . . . 9

1.3. Exoplanets . . . . . . . . . . . . . . . . . . . 11

1.3.1. Radial-velocity method . . . . . . . . . . . . . . . 13

1.3.2. CARMENES . . . . . . . . . . . . . . . 14

1.4. Stellar activity . . . . . . . . . . . . . . . . 16

1.4.1. Observed phenomena . . . . . . . . . . . . . . 16

1.4.2. Origin of stellar activity .................. 24

1.4.3. Implications for exoplanet surveys . . . . . . . . . . . 26

1.4.4. Outline ...................... . . 27

2. Data and methods 29

2.1. CARMENES spectra . . . . . . . . . . . . . . . . . . 29

2.2. Strengths of chromospheric lines . . . . . . . . . . . . . . 31

2.2.1. Grouping of stars and selection of reference stars . . . . . . . . 34

2.2.2. Normalised $\mathrm{H} \alpha$ luminosity . . . . . . . . . . . . . . . . . . . . 37

2.3. Indices of photospheric bands . . . . . . . . . . . . . . . . 38

3. Chromospheric indicators 4

3.1. Sample overview . . . . . . . . . . . . . . . . . . 41

3.2. Correlations . . . . . . . . . . . . . . . . . 44

3.3. Temporal variability . . . . . . . . . . . . . . . . . . . . . . . . . . . . . . 47

3.4. Impact of reference stars . . . . . . . . . . . . . . . 50

3.5. Discussion . . . . . . . . . . . . . . 53

4. Photospheric indicators $\quad 59$

4.1. Sample overview . . . . . . . . . . . . . . . . . . . 59

4.2. Correlations .......................... 61

4.2.1. Correlations among band indices . . . . . . . . . . . . 61 
4.2.2. Correlations with $\mathrm{H} \alpha \ldots \ldots 63$

4.3. Temporal variability . . . . . . . . . . . . . . . . . . 64

4.4. Discussion . . . . . . . . . . . . . . . . . . . 66

5. Rotational variation of activity indicators $\quad \mathbf{7 1}$

5.1. Sample overview . . . . . . . . . . . . . . . 71

5.2. Four individual stars . . . . . . . . . . . . . 73

5.3. Discussion . . . . . . . . . . . . . . . 78

6. Summary and conclusion 81

A. Table of stars $\quad 85$

$\begin{array}{ll}\text { B. Periodograms } & 95\end{array}$

$\begin{array}{ll}\text { Bibliography } & 105\end{array}$ 


\section{List of Figures}

1.1. CARMENES spectrum of Luyten's star . . . . . . . . . . . . . . . . . . 4

1.2. Schematic view of a Hertzsprung-Russell diagram . . . . . . . . . . . 7

1.3. Spectral radiance of black bodies with different effective temperatures . . 8

1.4. Energy transfer mechanisms in stellar interiors . . . . . . . . . . . . . . 10

1.5. Cross-section of the Sun . . . . . . . . . . . . . . . . . 11

1.6. Mass-period diagram of confirmed exoplanets . . . . . . . . . . . . . . 12

1.7. Illustration of the radial-velocity method . . . . . . . . . . . . . . . 13

1.8. Extracted CARMENES spectrum of Luyten's star . . . . . . . . . . . . . 15

1.9. Distribution of CARMENES sample stars on the sky . . . . . . . . . . 15

1.10. Line profile distortion by a starspot . . . . . . . . . . . . . . . . . 17

1.11. Sector of the solar disc at different wavelengths . . . . . . . . . . . . . . 19

1.12. H $\alpha$ line in spectra of EV Lac during and after flares . . . . . . . . . . . 22

1.13. Normalised $\mathrm{H} \alpha$ luminosity as function of rotation period and fraction of $\mathrm{H} \alpha$ active stars per spectral type for Carmencita stars . . . . . . . . 25

2.1. Number of stars with a given number of analysed spectra . . . . . . . . 30

2.2. Chromospheric lines . . . . . . . . . . . . . . 32

2.3. rms of $\mathrm{PC}$ ranges in residual spectra around $\mathrm{H} \alpha$ for $\mathrm{M} 3.0$ stars . . . . . . 36

2.4. Deviations between spectral types from minimum $r m s$ and Carmencita . . 37

2.5. Photospheric bands . . . . . . . . . . . . . . . . . 40

3.1. $\mathrm{pEW}^{\prime}$ of chromospheric lines as a function of spectral type . . . . . . . 42

3.2. Normalised $\mathrm{H} \alpha$ luminosity and fraction of $\mathrm{H} \alpha$ active stars per spectral type 43

3.3. Scatter plots of $\mathrm{H} \alpha$ versus other chromospheric lines . . . . . . . . . . . 45

3.4. Scatter plots of He lines and Ca lines . . . . . . . . . . . . . . . . 46

3.5. Relative $\mathrm{pEW}^{\prime}$ variations as functions of normalised $\mathrm{H} \alpha$ luminosity . . . 48

3.6. Absolute $\mathrm{H} \alpha$ variation as a function of $\mathrm{pEW}_{\mathrm{H} \alpha}^{\prime} \ldots \ldots$. . . . . . . . . . . . 49

3.7. Differences between $\mathrm{pEW}^{\prime}$ values calculated with different reference stars 51

3.8. Scatter plots of $\mathrm{pEW}^{\prime}$ variations calculated with different reference stars . 52

3.9. Comparison of $\mathrm{pEW}^{\prime}$ values with earlier work . . . . . . . . . . . . . 54

3.10. Comparison of $\mathrm{pEW}^{\prime}$ values with $\mathrm{pEW}$ values from literature . . . . . . 55

3.11. $v \sin i, P_{\text {rot }}$, and magnetic field strength as functions of normalised $\mathrm{H} \alpha$ luminosity, and $\mathrm{H} \alpha$ active stars per stellar population . . . . . . . . 56

4.1. Photospheric absorption band indices as a function of spectral type . . . . 60

4.2. Scatter plots of TiO 7050 versus other photospheric bands . . . . . . . 62 
4.3. Scatter plots of $\mathrm{CaH}$ bands and $\mathrm{VO}$ bands . . . . . . . . . . . . 63

4.4. Scatter plots of photospheric bands with polynomial fits to reference stars 63

4.5. Distances from inactivity line as functions of normalised $\mathrm{H} \alpha$ luminosity . 64

4.6. Relative index variations as functions of index values . . . . . . . . . 65

4.7. Comparison of photospheric band indices with earlier work . . . . . . . 66

4.8. Comparison of photospheric band indices with indices from literature . . 68

4.9. Scatter plots of photospheric bands colour-coded by $T_{\mathrm{eff}}$ and $[\mathrm{Fe} / \mathrm{H}] \quad \ldots \quad 69$

5.1. Fraction of stars showing the rotation frequency per activity indicator . . 72

5.2. $\log p\left(1 / P_{\text {rot }}\right)$ and $\log p\left(2 / P_{\text {rot }}\right)$ as a function of BJD for Ross $318 \ldots . .76$

5.3. $\log p\left(1 / P_{\text {rot }}\right)$ and $\log p\left(2 / P_{\text {rot }}\right)$ as a function of BJD for YZ CMi . . . . 76

5.4. $\log p\left(1 / P_{\text {rot }}\right)$ and $\log p\left(2 / P_{\text {rot }}\right)$ as a function of BJD for TYC 3529-1437-1 77

5.5. $\log p\left(1 / P_{\text {rot }}\right)$ and $\log p\left(2 / P_{\text {rot }}\right)$ as a function of BJD for EV Lac . . . . . 77

5.6. Fractions of sets of indicators that show a significant signal at $1 / P_{\text {rot }} \ldots 79$

B.1. GLS periodograms for Ross $318 \ldots \ldots$. . . . . . . . . . 96

B.2. GLS periodograms for $\mathrm{YZ} \mathrm{CMi} \mathrm{\ldots .} \mathrm{.} \mathrm{.} \mathrm{.} \mathrm{.} \mathrm{.} \mathrm{.} \mathrm{.} \mathrm{.} \mathrm{.} \mathrm{.} \mathrm{.} \mathrm{.} \mathrm{.} \mathrm{.} 97$

B.3. GLS periodograms for TYC 3529-1437-1 . . . . . . . . . . . . . . . 98

B.4. GLS periodograms for EV Lac . . . . . . . . . . . . . . . . . . . 99

B.5. Rolling GLS periodograms for Ross 318 . . . . . . . . . . . . . . . 100

B.6. Rolling GLS periodograms for YZ CMi . . . . . . . . . . . . . . . . 101

B.7. Rolling GLS periodograms for TYC 3529-1437-1 . . . . . . . . . . . . . 102

B.8. Rolling GLS periodograms for EV Lac . . . . . . . . . . . . . . . . 103 


\section{List of Tables}

1.1. Effective temperatures corresponding to spectral subtypes . . . . . . . 8

2.1. Line windows and pseudo-continuum ranges for $\mathrm{pEW}^{\prime}$ measurements of chromospheric lines . . . . . . . . . . . . . . . 33

2.2. Reference stars for each spectral subtype . . . . . . . . . . . . . 35

2.3. Numerator and denominator ranges for photospheric absorption band indices . . . . . . . . . . . . . . . . . . . 39

3.1. Correlation table of chromospheric lines . . . . . . . . . . . . . . . 44

3.2. Correlation table of chromospheric lines for $\mathrm{H} \alpha$ active stars . . . . . . . . 47

4.1. Correlation table of photospheric bands . . . . . . . . . . . . 61

5.1. Basic parameters and number of CARMENES observations of four stars . 73

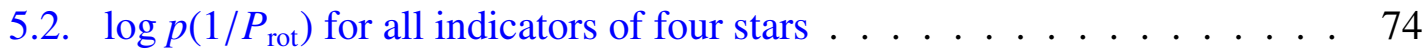

A.1. Identifications, basic parameters, and $\mathrm{H} \alpha$ results of the analysed stars . . . 86 



\section{Introduction}

This chapter gives an overview of the physical background for this thesis: While Sect. 1.1 introduces spectroscopy as the concept for collecting the information analysed in this thesis from the stars, Sects. 1.2 and 1.3 outline how stars are classified and how their planets are discovered. This aims to explain the terms M dwarf and CARMENES used in the title of this thesis. Stellar activity is explained in Sect. 1.4 with a description of the observed phenomena, their physical interpretation, and their relevance in the context of $\mathrm{M}$ dwarfs and exoplanets. The remaining chapters of this thesis are outlined in Sect. 1.4.4.

\subsection{Spectroscopy}

The spectrum of a light source is given by the flux as a function of the wavelength $\lambda$ or the frequency $v$. As an example, I consider a body with a constant temperature $T$ that completely absorbs any incoming radiation. Such a body is called a black body and emits light with a continuous spectrum that is described by Planck's law. The spectral radiance per unit wavelength depends on $T$ and is given by

$$
B_{\lambda}(\lambda, T)=\frac{2 h c^{2}}{\lambda^{5}} \frac{1}{\exp \left(\frac{h c}{\lambda k_{B} T}\right)-1},
$$

where $h$ is the Planck constant, $c$ is the speed of light, and $k_{B}$ is the Boltzmann constant. At any given wavelength $\lambda, B_{\lambda}$ is higher for a black body with a higher temperature. However, the wavelength $\lambda_{\max }$ where $B_{\lambda}$ is maximum increases with decreasing temperature as given by Wien's displacement law:

$$
\lambda_{\max } T=2.897771955 \cdot 10^{-3} \mathrm{~m} \mathrm{~K}
$$

The total emitted energy per unit time of a body is called its luminosity. For a black body with radius $R$ and temperature $T$, the luminosity $L$ follows from integration of Planck's law over all wavelengths and the body's surface, and is given by the Stefan-Boltzmann law:

$$
L=4 \pi R^{2} \sigma T^{4},
$$

where $\sigma$ is the Stefan-Boltzmann constant. This relation can also be used to define an effective temperature $T_{\text {eff }}$ of a body that is not a perfect black body. 


\subsubsection{Spectral lines}

With its emitted light, each star sends out its fingerprint. The radiation generated in the core passes through the stellar atmosphere that can contain a variety of atoms and molecules in various states. Photons with an energy that corresponds to the difference between two energy levels of a molecular entity can be absorbed. The entity is then in an excited state and loses the energy either in a collision or by re-emitting a photon in a random direction. An observer now sees an absorption line in the spectrum, as there is less flux at the wavelength corresponding to the absorbed photon's energy. Similarly, molecular entities can be excited by other mechanisms and then emit additional photons with a specific energy. The observer now sees an emission line in the spectrum. As the wavelengths of absorption or emission lines can be compared with theoretical calculations or experimental results, the spectral lines allow to identify atoms or molecules that are present in the star's atmosphere. Because of their rotational and vibrational energy levels, molecules can absorb photons with similar energies, leading to a forest of lines rather than a single absorption line. This is called an absorption band.

Several effects cause a spectral line to appear broader than an infinitesimally narrow line. From the limited lifetime of an excited state follows an uncertainty in the energy according to Heisenberg's uncertainty principle. This natural broadening can be further increased by a higher pressure that leads to more collisions and thus faster collisional de-excitation and shorter effective lifetimes of the excited state. Additionally, the molecular entities in the stellar atmosphere move with different velocities according to a Maxwell distribution related to the temperature. Therefore, some entities move towards and other entities move away from the observer, causing different Doppler shifts resulting in thermal broadening. Similarly, the entities can move with different velocities with respect to the observer as the star rotates, causing rotational broadening. Further broadening can be caused by electrical or magnetic fields, e.g. via the Zeeman effect. While all these broadening mechanisms take place in the stellar atmosphere and affect the real line shapes, the instrument used to observe the spectrum will also affect the observed line shapes.

\subsubsection{Spectrographs}

Before a stellar spectrum can be analysed, the wavelength dependence of the light emitted by the star has to be measured. Most commonly, this is done by dispersing the light with a prism, a grating, or a grism, although Fourier transform spectrocopy with interferometers emerged as an alternative in the second half of the twentieth century (Loewenstein 1966). Prisms were used for the first spectral observations of the Sun and the brightest stars by Isaac Newton in the late seventeenth century and for the discovery of absorption lines by William Hyde Wollaston and Joseph von Fraunhofer in the early nineteenth century, whereas gratings were first used by Thomas Young in 1801 for solar observations, but were not useful for stellar observations before the development of the blazed grating in 
the early twentieth century (Hearnshaw 2009, 2014). After Jacquinot (1954) had demonstrated the superiority of blazed gratings, prisms widely disappeared from astrophysical spectroscopy, but returned in combination with gratings in form of the grism (Bowen \& Vaughan 1973).

The smallest wavelength difference $\Delta \lambda$ that can be resolved by a spectrograph varies with the wavelength range. Therefore, the resolution of a spectrograph is usually quantified by the resolving power $R$. For a grating with $N$ illuminated grooves, the resolving power in the $m$ th spectral order is given by

$$
R=\frac{\Delta \lambda}{\lambda}=m N
$$

Using the grating equation

$$
m \lambda=\frac{W}{N}(\sin \alpha+\sin \beta),
$$

where $W$ is the spacing of the grooves, $\alpha$ the angle of incidence and $\beta$ the angle of diffraction, $N$ can be eliminated as pointed out by Harrison (1949). For a blazed grating with blaze angle $\theta_{\mathrm{B}}$, the angles of incidence and diffraction are given by $\alpha=\theta_{\mathrm{B}}+\theta$ and $\beta=\theta_{\mathrm{B}}-\theta$. In quasi-Littrow mode, the diffracted light travels back along the path of the incident light, so $\theta \approx 0$. The resolving power is then given by

$$
R=\frac{2 W \sin \theta_{\mathrm{B}}}{\lambda} .
$$

Therefore, high-resolution spectrographs use gratings with high blaze angles such as the reflective echelle gratings. Modern echelle spectrographs usually follow the design of the ELODIE spectrograph (Baranne et al. 1996) that has a blaze angle of $\theta_{\mathrm{B}}=76^{\circ}$. In an echelle spectrograph, the light diffracted by an echelle grating is cross-dispersed to separate the spectral orders. This leads to a two-dimensional spectrum such as Fig. 1.1.

To calibrate the wavelength corresponding to a position in the two-dimensional spectrum, spectral lines with known wavelengths have to be observed. On its way to the observer, parts of the stellar light are absorbed by molecules in the Earth's atmosphere causing telluric absorption lines in the spectrum. Particularly in the near-infrared range, strong telluric absorption bands can cover significant parts of a spectral order, as can be seen in Fig. 1.1. The wavelengths of telluric lines are known from models and can thus be used to calibrate the spectrum (e.g. Griffin \& Griffin 1973; Seifahrt et al. 2010). Similarly, the stellar light can be passed through an absorption gas cell that superimposes absorption lines with known wavelengths before entering the spectrograph (e.g. Campbell \& Walker 1979; Marcy \& Butler 1992). Alternatively, the spectrum of a light source with known emission lines can be observed, either before and after the stellar spectrum or in parallel. A common light source used for wavelength calibration of echelle spectrographs are hollow cathode lamps (e.g. Kerber et al. 2007; Sarmiento et al. 2018). Because the observable spectral lines are inherent to the molecular entities that absorb or emit the light, these techniques may provide too few or too sparsely distributed lines in parts of the covered wavelength range. In contrast, Fabry-Pérot interferometers (e.g. Wildi et al. 2010; Bauer 


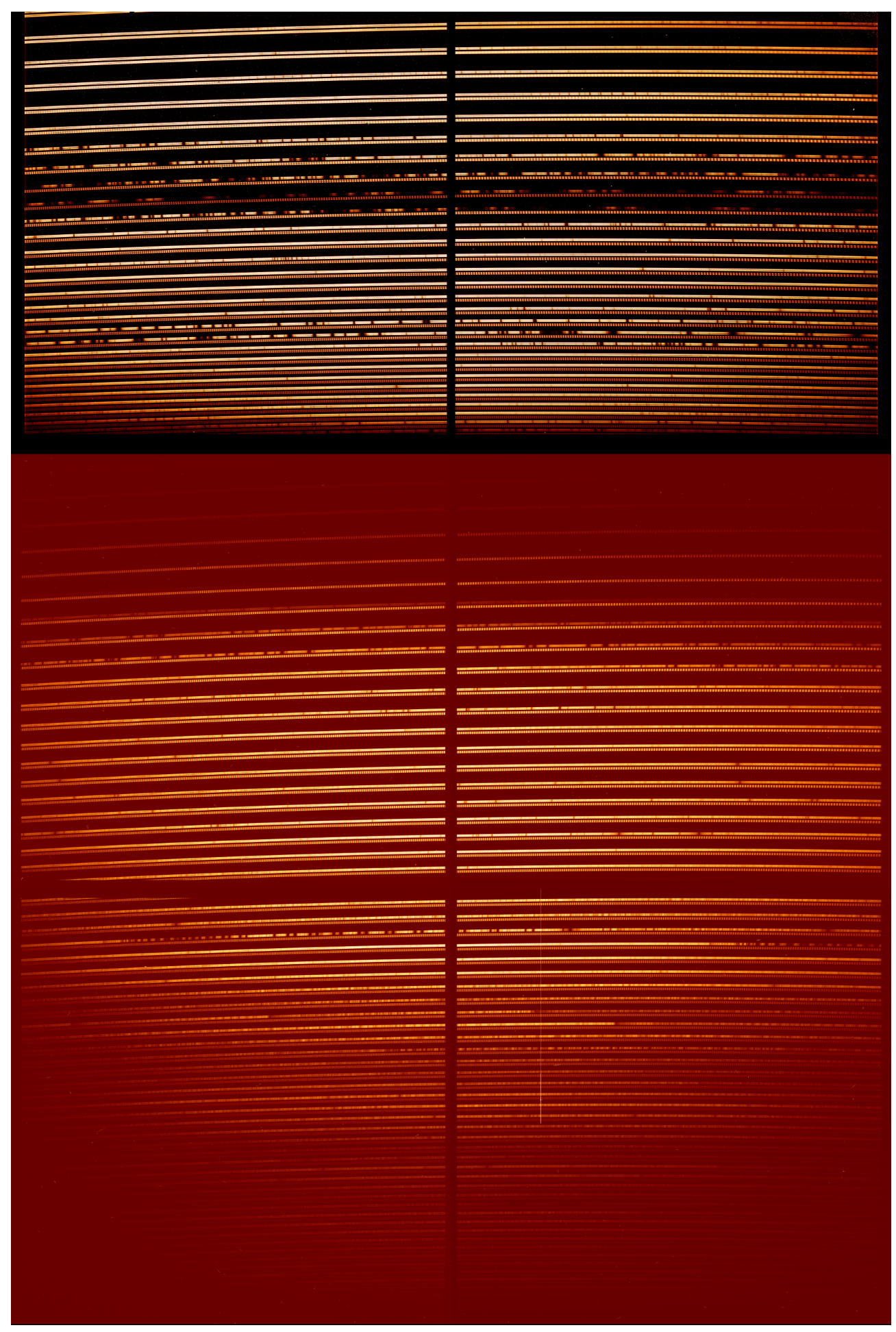

Figure 1.1.: CARMENES spectrum of Luyten's star on 25 February 2016 at 21:07:51 UT with Fabry-Pérot etalon spectrum for radial-velocity drift correction. The upper third is from the nearinfrared channel (9600-17100 $)$ ), the two bottom thirds are from the visible-light channel (5200$9600 \AA$ ) of the spectrograph. 
et al. 2015) and laser frequency combs (e.g. Murphy et al. 2007; Metcalf et al. 2019) provide a grid of evenly spaced lines in wavelength and frequency space, respectively, and can thus significantly increase the precision of the wavelength calibration.

\subsection{Star classification}

Already in ancient times, stars were classified by their apparent brightness. With a revised definition, the ancient system of magnitudes is still in use today. Another difference between stars that is apparent to the naked eye in some cases is their colour. The apparent colour depends on the wavelength of maximum flux, which according to Eq. 1.2 depends on the effective temperature. A classification by colour is therefore the same as a classification by temperature.

\subsubsection{Spectral types}

Another approach that turns out to be equivalent to a classification by colour or temperature is a classification by spectral features. According to Eq. 1.1, there are relatively few photons of short wavelengths and high energies in cool stars, and thus they cannot be absorbed. However, while photons of all wavelengths and energies are more common in hot stars, the atoms can be in higher energy states or ionised and molecules can be broken up. In thermal equilibrium, molecular entities of the same species occupy different energy states and atoms appear in different ionisation states according to temperature-dependent ratios. Therefore, presence and strength of spectral lines are indicative for the temperature of the star.

First classifications based on the strength of hydrogen lines were done by Angelo Secchi and Williamina Fleming in the late nineteenth century (Giridhar 2010). Fleming used the classes A to $\mathrm{N}$ with decreasing strength of hydrogen lines. Cannon \& Pickering (1901) discarded some classes and reordered the remaining classes to the sequence of spectral types $\mathrm{O}, \mathrm{A}, \mathrm{B}, \mathrm{F}, \mathrm{G}, \mathrm{K}$, and $\mathrm{M}$, with intermediate types such as "A 5 F". With further modifications by Morgan et al. (1943) and Johnson \& Morgan (1953), and the addition of the spectral types L, T, and Y (Kirkpatrick et al. 1999; Burgasser et al. 2006), this system is still used today. As it was earlier believed that the hot stars are young and the cool stars are old, spectral types are traditionally called early (towards $\mathrm{O}$ ) and late (towards Y).

\subsubsection{Luminosity classes}

Scatter plots of the classification by apparent brightness and the classification by colour, temperature, or spectral type, are called colour-magnitude diagrams. Using the absolute 
magnitude or the luminosity $L$ as defined in Sect. 1.1 instead of the apparent brightness yields the Hertzsprung-Russell diagram (HRD) that was originally described by Hertzsprung (1911) and Russell (1913) and was crucial to understand how stars evolve. A schematic view of a HRD is shown in Fig. 1.2.

Most stars appear on a line extending from hot and bright to cool and faint. This line is called the main sequence. However, there are groups of outliers above and below the main sequence. For a fixed temperature, the luminosity can, according to Eq. 1.3, only change if the radius changes. Therefore, the stars above the main sequence must have larger radii than main-sequence stars with the same temperature and are called giants. In contrast, the main-sequence stars are also called $d$ warfs. While a star spends most of its life as a dwarf, it does not move much on the main sequence. Giants are in a later state of stellar evolution. Based on the different regions in the HRD, the spectral type definition by Johnson \& Morgan (1953) also includes luminosity classes denoted by Roman numbers. Main-sequence stars or dwarfs are luminosity class V. As only dwarfs are analysed in this thesis, I generally leave out the luminosity class when specifying spectral types and give the full spectral type with luminosity class only in Table A.1.

\subsection{3. $M$ dwarfs}

Combining the classifications from two previous sections, stars with spectral type $\mathrm{M}$ and luminosity class $\mathrm{V}$ are called $M d w a r f s$, sometimes abbreviated as $\mathrm{dM}$. In the original classification by Cannon \& Pickering (1901), M was the latest spectral type and defined by the clear presence of titanium oxide absorption bands in the spectrum. While this classification was based on visible-light spectra, Kirkpatrick et al. (1991) defined standard stars for spectral subtypes between $\mathrm{K} 5$ and $\mathrm{M} 9$ using $\mathrm{TiO}$ and $\mathrm{VO}$ absorption bands in near-infrared spectra. Pecaut \& Mamajek (2013) created a table of the typical effective temperature from $T_{\text {eff }}$ measurements and estimates of the most common standard stars for each spectral subtype. I list their effective temperatures for $\mathrm{M}$ dwarf subtypes in Table 1.1. For M7.5 and M8.5, I give the average effective temperatures of M7.0 and M8.0, and of M8.0 and M9.0, respectively. Using these effective temperatures and Eq. 1.2, the wavelength of maximum flux for M dwarfs is between around $7500 \AA$ for M0.0 and $12100 \AA$ for M9.5. As illustrated in Fig. 1.3, M dwarfs thus emit most of their light at near-infrared wavelengths.

M dwarfs cover a wide range of fundamental stellar parameters (in the following, the subscript $\odot$ refers to solar units): Masses range from $0.6 M_{\odot}$ for $\mathrm{M} 0$ down to $0.075 M_{\odot}$ for M9 (Reid \& Hawley 2005), which is the minimum mass required to sustain hydrogen burning in the core (Chabrier \& Baraffe 2000). Because of their low mass, M dwarfs of various ages can be observed, as even the first formed $M$ dwarfs have not yet evolved beyond the main sequence with lifetimes up to $10^{13}$ years (Laughlin et al. 1997). Typical

\footnotetext{
${ }^{1}$ https: //www . eso.org/public/images/eso0728c/
} 


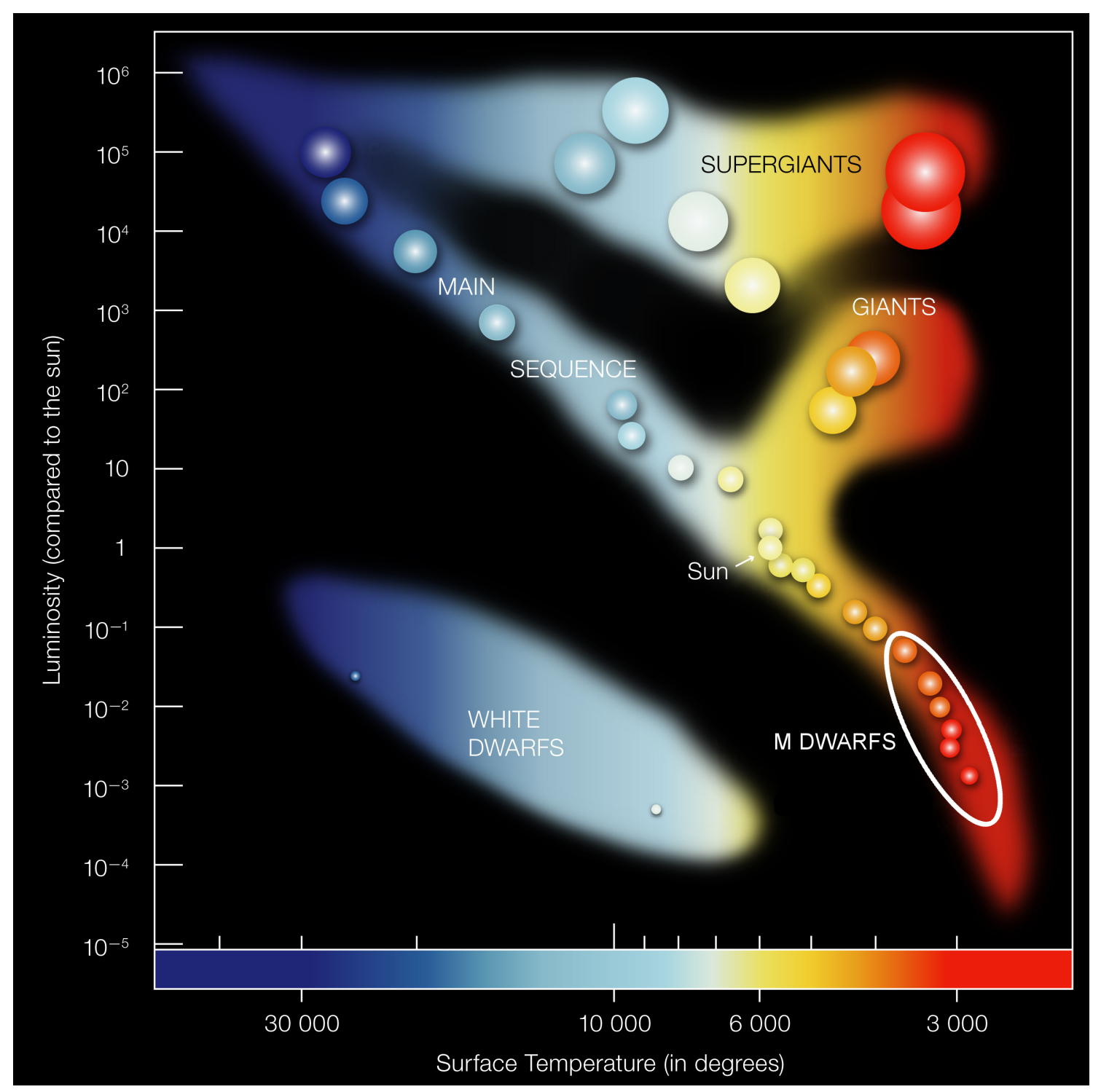

Figure 1.2.: Schematic view of a Hertzsprung-Russell diagram. Image credit: adapted from $\mathrm{ESO}^{1}$, used under CC-BY 4.0 
Table 1.1.: Effective temperatures corresponding to spectral subtypes as compiled by Pecaut \& Mamajek (2013).

\begin{tabular}{ccccc}
\hline Spectral type & $T_{\text {eff }}[\mathrm{K}]$ & & Spectral type & $T_{\text {eff }}[\mathrm{K}]$ \\
\cline { 1 - 2 } \cline { 5 - 5 } M0.0 & 3850 & & M5.0 & 3050 \\
M0.5 & 3800 & & M5.5 & 3000 \\
M1.0 & 3680 & & M6.0 & 2800 \\
M1.5 & 3600 & & M6.5 & 2700 \\
M2.0 & 3550 & M7.0 & 2650 \\
M2.5 & 3450 & & M7.5 & 2610 \\
M3.0 & 3400 & & M8.0 & 2570 \\
M3.5 & 3250 & & M8.5 & 2510 \\
M4.0 & 3200 & M9.0 & 2450 \\
M4.5 & 3100 & M9.5 & 2400 \\
\hline
\end{tabular}

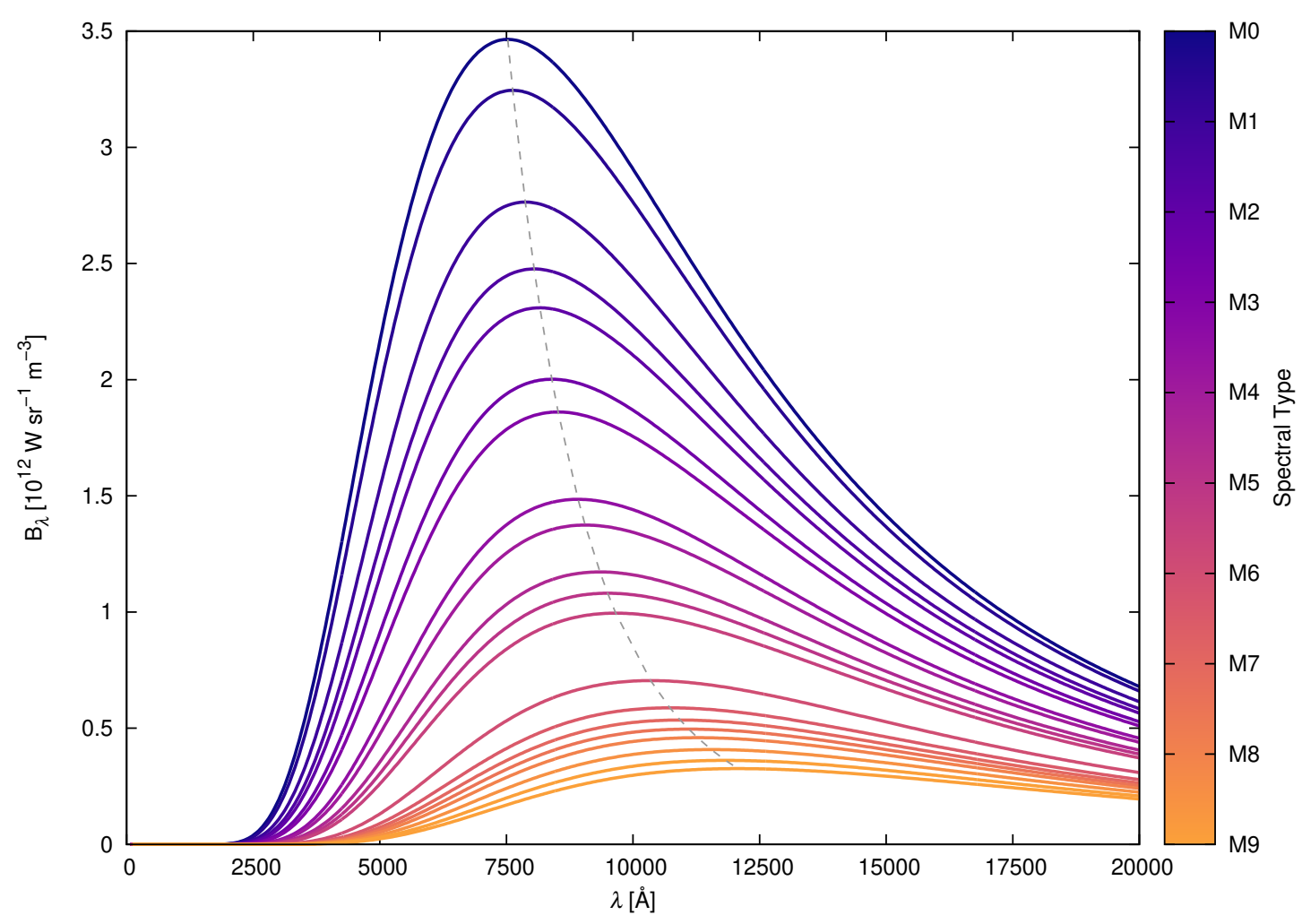

Figure 1.3.: Spectral radiance per unit wavelength of black bodies with effective temperatures corresponding to different $\mathrm{M}$ subtypes as listed in Table 1.1 according to Eq. 1.1. The dashed grey line connects the maxima of the curves at the wavelengths given by Eq. 1.2. 
radii between $0.08 R_{\odot}$ for $\mathrm{M} 9$ and $0.62 R_{\odot}$ for M0 correspond to typical luminosities between $1.5 \cdot 10^{-4} L_{\odot}$ for M9 and 7.2 $\cdot 10^{-2} L_{\odot}$ for M0 (Reid \& Hawley 2005). Therefore, $\mathrm{M}$ dwarfs are very faint stars, and even Proxima Centauri, at a distance of $1.301 \mathrm{pc}$ (Gaia Collaboration et al. 2018) the closest stellar neighbour of the Sun and an M dwarf with spectral type M5.5 (Boyajian et al. 2012), is invisible to the naked eye from Earth, as are all other M dwarfs. Still, with $283 \mathrm{M}$ dwarfs among 378 known stars within 10 pc (Henry et al. 2018, RECONS ${ }^{2}$ ), they are the most common type of stars in the solar neighbourhood and planets occur more frequently at later spectral types (Mulders et al. 2015; Gaidos et al. 2016; Hardegree-Ullman et al. 2019), making this class of stars an interesting field of research.

\subsubsection{Structure and atmospheres of main-sequence stars}

Low-mass stars at the red end of the main sequence are not simply scaled-down versions of hotter and more massive main-sequence stars, but differ in the way energy is produced in their cores and transported outwards. While the energy source of all main-sequence stars is the fusion of hydrogen into helium, the dominant process in hot early-type dwarfs is the CNO cycle, whereas the proton-proton chain dominates in Sun-like and later-type dwarfs (e.g. Adelberger et al. 2011). The dominant energy transfer mechanisms are convection and radiation. Convection is most efficient in regions with a steep temperature gradient, whereas radiation does not require a temperature gradient, but works best if the opacity is low. In stars with masses above $1.5 M_{\odot}$ or spectral type A and earlier, the extreme temperature dependence of the $\mathrm{CNO}$ cycle constrains the energy production to a relatively small inner core. This leads to a steep temperature gradient above the inner core, and thus a convection zone in the inner part, while radiation is dominant in the outer part, where the temperature gradient is shallower. For Sun-like stars with masses below $1.5 M_{\odot}$, the energy production via proton-proton chain is more distributed and does not lead to a sufficient temperature gradient for efficient convection. Therefore, the inner part is radiative. However, in the outer part, the temperature is sufficiently low for the recombination of hydrogen atoms. This results in a higher opacity, and convection becomes more efficient than radiation in this part. The transition layer between the inner radiation and the outer convection zones is called tachocline. With decreasing mass and temperature, the outer convection zone becomes deeper until the inner radiation zone vanishes completely at around $0.3 M_{\odot}$ or spectral type M4 (Chabrier \& Baraffe 1997; Stassun et al. 2011). Later M dwarfs are thus fully convective. The different internal structures of stars along the main sequence are summarised in Fig. 1.4.

While the stellar interior contributes most to the stellar mass, it is optically thick and can thus not be observed directly. Photons can only escape from the more transparent outer layers, collectively referred to as the stellar atmosphere. It is this region where spectral lines are formed as described in Sect. 1.1.1. As can be seen in the cross-section of the Sun

\footnotetext{
${ }^{2}$ http: //wWw. recons.org, census of objects nearer than $10 \mathrm{pc}$ as of 12 April 2018
} 


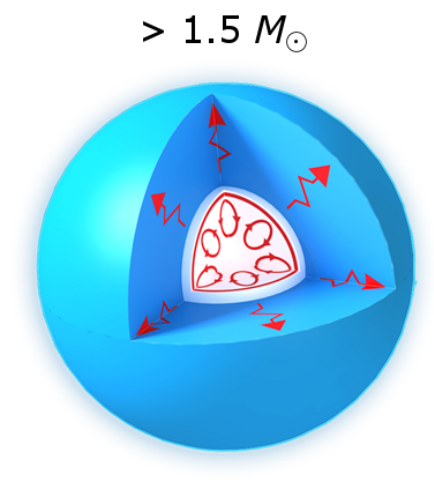

earlier than FO

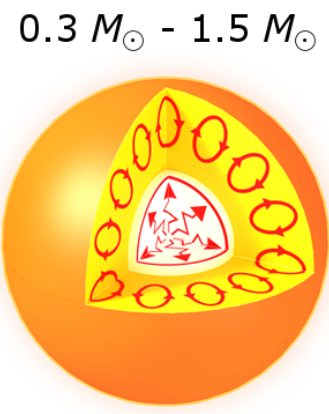

F0 - M4
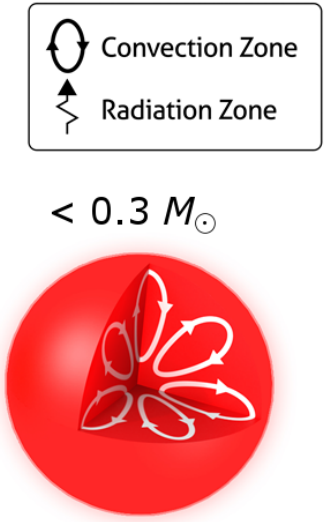

later than M4

Figure 1.4.: Energy transfer mechanisms in the interiors of stars with different masses and spectral types. Massive early-type stars have a convective core and an outer radiation zone, whereas Sunlike stars have a radiative core and an outer convection zone. Low-mass late $\mathrm{M}$ dwarfs are fully convective. Image credit: adapted from Sun.org - www.sun.org ${ }^{3}$, used under CC-BY-SA 3.0, modifications released under CC-BY-SA 4.0

in Fig. 1.5, three layers of stellar atmospheres are distinguished. Most of the light that finally reaches the observer originates from the lowest atmospheric layer, the photosphere. This layer therefore determines the observed effective temperature and spectral type of a star, while the upper atmospheric layers are less dense and usually outshined. Lockyer (1868) introduced the term chromosphere for the layer above the photosphere that appears red during a solar eclipse. Unlike in the photosphere, the temperature does not decrease with increasing height and decreasing pressure in the chromosphere (e.g. Fontenla et al. 2016). In the narrow transition region between chromosphere and corona, the temperature increases drastically (e.g. Mariska 1986). The corona, named after its appearance during solar eclipses, is the outermost and also hottest and least dense atmospheric layer. It can extend to several stellar radii as defined by the photosphere (e.g. Benz et al. 1998; Pestalozzi et al. 2000; Shopov et al. 2008). While a photosphere can be defined for every main-sequence star, chromospheres and coronae are ubiquitous among Sun-like and later-type stars, but not observed for spectral types earlier than late A (e.g. Simon et al. 2002). As these early-type stars do not have an outer convection zone in their interiors, this suggests that an outer convection zone is required for the formation of the hot outer atmospheric layers. I will describe observations and the heating of chromospheres and coronae in the context of stellar activity in Sect. 1.4.

\footnotetext{
${ }^{3}$ http: //www.sun.org/images/heat-transfer-in-stars
} 


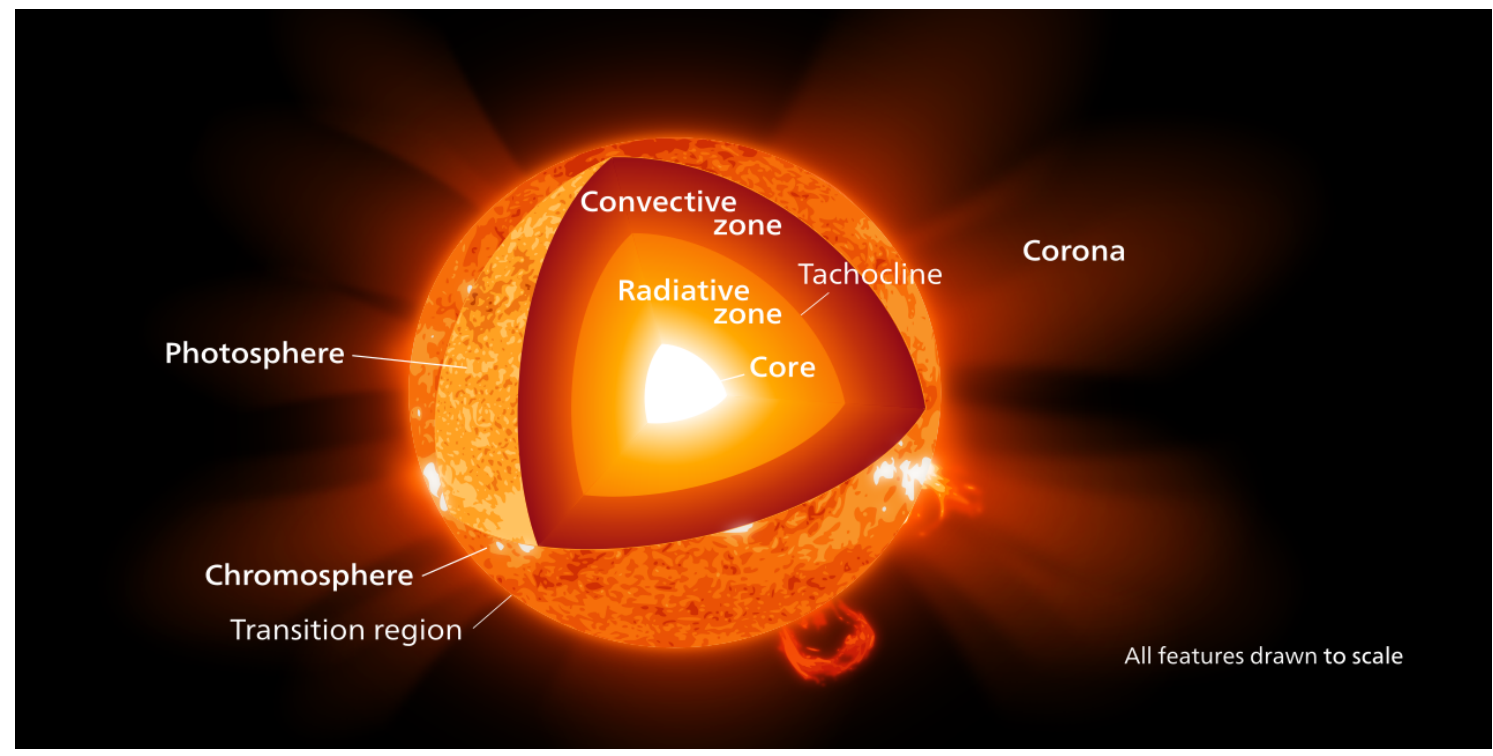

Figure 1.5.: Cross-section of the Sun with marked interior and atmospheric layers. Image credit: adapted from Kelvinsong via Wikimedia Commons ${ }^{4}$, used under CC-BY-SA 3.0, modifications released under CC-BY-SA 4.0

\subsection{Exoplanets}

One of the first recorded methods to detect planets around other stars was already proposed in the nineteenth century by Niven (1874), but it took more than a century until the first successes. While the first confirmed exoplanet orbiting a pulsar was discovered by Wolszczan \& Frail (1992), three years later, Mayor \& Queloz (1995) discovered the first exoplanet around a main-sequence star and were awarded the Nobel prize for this discovery in 2019. Several methods to detect exoplanets have been used successfully since then:

- Transit method: A transiting planet blocks out a small fraction of the stellar light as it passes in front of the star, causing periodic dips in the light curve.

- Radial-velocity method: As the star moves around the common centre of mass of the system, a periodic modulation in its radial velocity is observed.

- Direct imaging: An image of the planet itself is obtained.

- Microlensing: Star and planet act as gravitational lenses on the light of a background source. The observed effect is different than the effect of a star alone.

- Timing variations: The star emits a periodic signal (e.g. a pulsar, an eclipsing binary, a pulsating star, or a transiting planet). While the star moves around the centre of mass of the system, its light has to travel a variable distance to the observer, and the periodic signal is observed earlier or later than expected.

\footnotetext{
${ }^{4}$ https://commons.wikimedia.org/wiki/File:Sun_poster.svg
} 


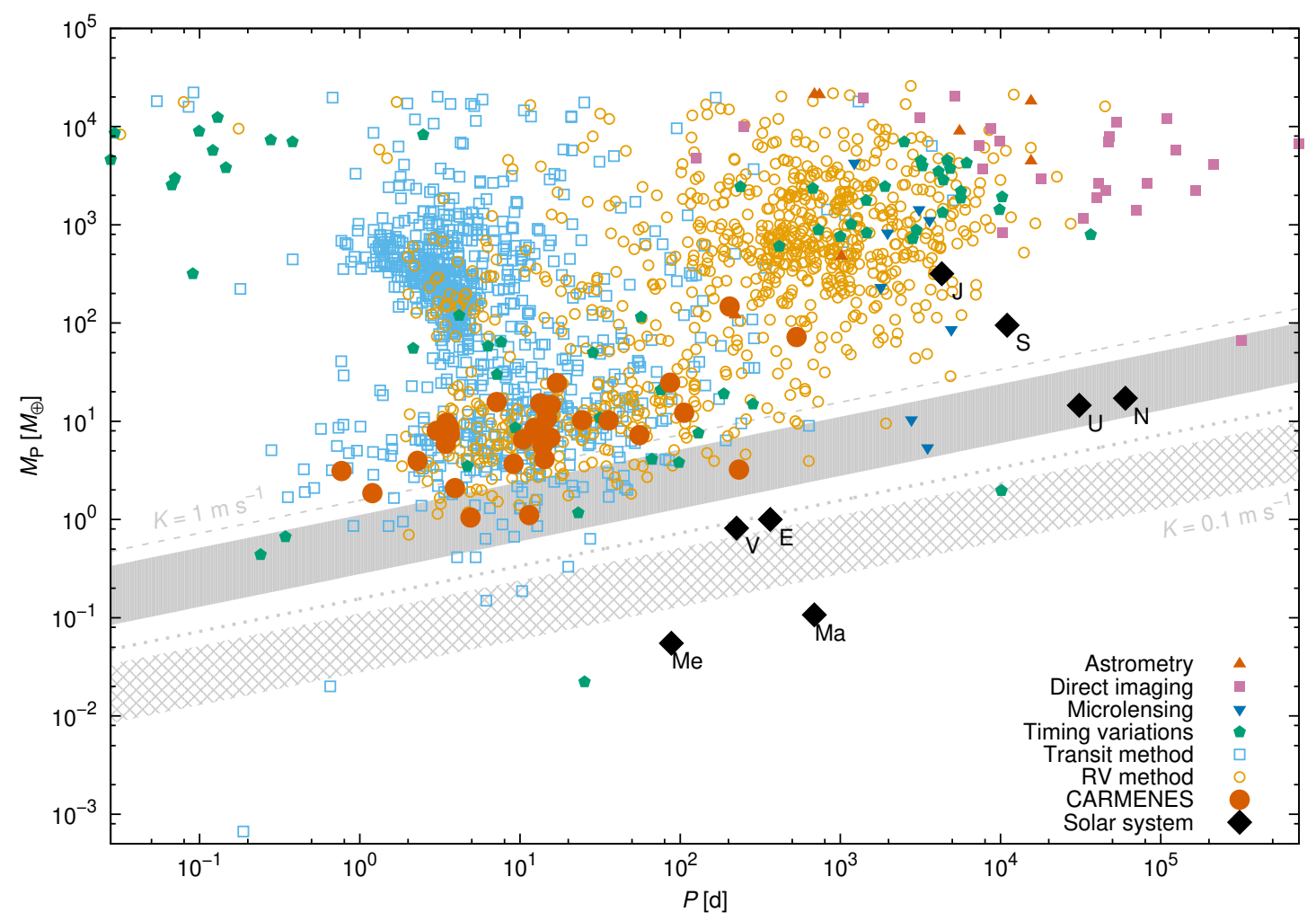

Figure 1.6.: Mass-period diagram of confirmed exoplanets using data from The ExtRasolar Planets Encyclopaedia ${ }^{5}$ as of 15 October 2020. Only planets with known orbital period $P$ and mass $M_{\mathrm{P}}$ or minimum mass $M_{\mathrm{P}} \sin i$ are shown. Masses are given in Earth masses $M_{\oplus}$. Different symbols correspond to different detection methods, the solar system planets are shown for comparison as black diamonds with their first letters. Exoplanets discovered by CARMENES are highlighted by red circles. The dashed grey line and the grey area mark the detection limits of the radial-velocity method with a semi-amplitude $K=1 \mathrm{~m} \mathrm{~s}^{-1}$ for a solar-mass star and the M dwarf mass range, respectively, whereas the dotted grey line and the hatched area show the same but for $K=0.1 \mathrm{~m} \mathrm{~s}^{-1}$.

- Astrometry: As the star moves around the common centre of mass of the system, periodic changes of its position are measured.

While the radial-velocity method is described in the following section, I refer to Perryman (2018) for more details on the other detection methods. As can be seen in the mass-period diagram in Fig. 1.6, the astrometric and direct imaging methods are most sensitive for massive planets with orbital periods of several years because these planets show the largest angular separations from their host stars and have the highest impact on the common centre of mass of the system. Microlensing also works best for long-period planets, but can detect lower-mass planets. The radial-velocity, timing variations, and transit methods cover a larger parameter space. With the exception of Jupiter, the solar system planets are located in sparsely populated regions of the diagram. Finding a solar system analogue would require observations with improved sensitivity over longer times. 

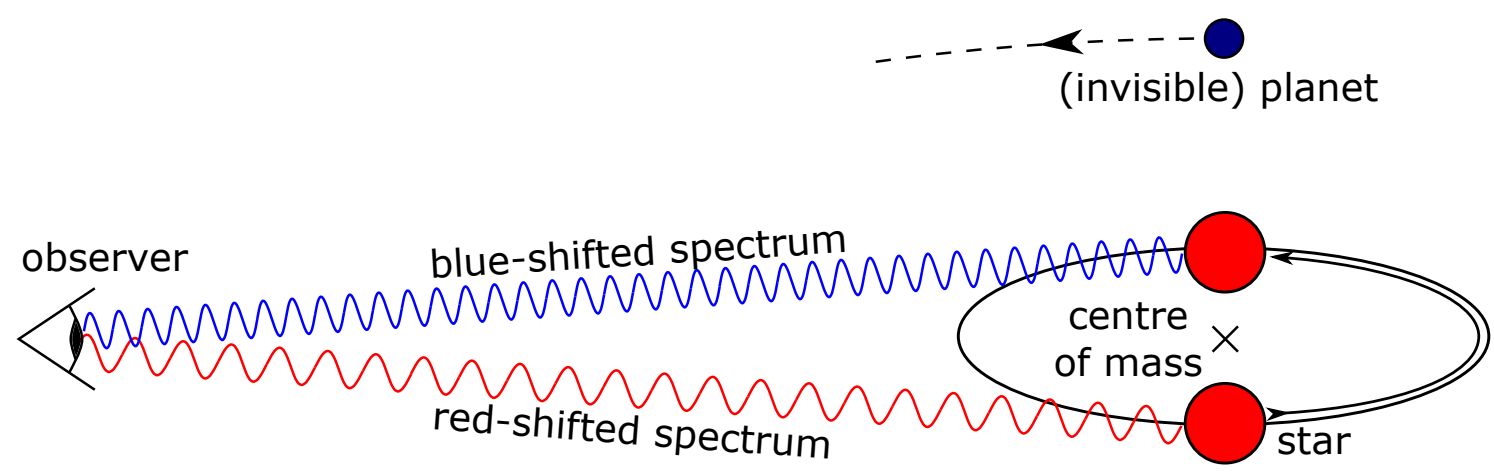

Figure 1.7.: Illustration of the idea behind the radial-velocity method: A planet and its host star orbit their common centre of mass. While the star moves away from the observer, its spectrum is red-shifted, whereas it is blue-shifted while the star moves towards the observer.

\subsubsection{Radial-velocity method}

A star that is orbited by a planet performs a motion around their common centre of mass. If the orbital plane is not perpendicular to the line of sight, the radial component of the velocity of the star with respect to the observer undergoes a periodic modulation. As illustrated in Fig. 1.7, the radial velocity (RV) and its variations can be measured from the Doppler shift of spectral lines:

$$
\frac{\mathrm{RV}}{c}=\frac{\lambda-\lambda_{0}}{\lambda_{0}},
$$

where $\mathrm{c}$ is the speed of light, $\lambda$ the observed wavelength and $\lambda_{0}$ the rest wavelength of the spectral line. If the star moves towards the observer, the RV is negative and $\lambda<\lambda_{0}$, so the spectrum appears blue-shifted. On the other hand, if the star moves away from the observer, the RV is positive and $\lambda>\lambda_{0}$, so the spectrum appears red-shifted. For a planet with mass $M_{\mathrm{P}}$ that orbits a star with mass $M_{\star}$ in a period $P$ on an orbit with numerical eccentricity $e$, the semi-amplitude $K$ of the RV modulation is given by

$$
K=\left(\frac{2 \pi G}{P}\right)^{\frac{1}{3}} \frac{M_{\mathrm{P}} \sin i}{\left(M_{\mathrm{P}}+M_{\star}\right)^{\frac{2}{3}}} \frac{1}{\sqrt{1-e^{2}}},
$$

where $G$ is Newton's constant and $i$ is the inclination angle between the orbital plane and a plane perpendicular to the line of sight. This angle is generally unknown, so only a minimum mass for the planet can be derived from the RV modulation, unless the planet can also be detected with the transit, direct imaging, or astrometric methods.

For an outside observer viewing the solar system edge-on, Eq. 1.8 yields a semi-amplitude $K \approx 0.09 \mathrm{~m} \mathrm{~s}^{-1}$ for the Sun caused by the Earth. This is comparable to the precision of the ESPRESSO spectrograph (Pepe et al. 2021) and below the detection thresholds of other current instruments, so Earth-like planets around Sun-like stars could not yet be found with the RV method. For a fixed planet mass, $K$ is larger for shorter orbital

\footnotetext{
${ }^{5}$ http: //exoplanet.eu
} 
periods $P$ and smaller stellar masses $M_{\star}$. This makes $\mathrm{M}$ dwarfs suitable targets for the search for Earth-like planets in the liquid water habitable zone. Because of their low temperatures and luminosities, the habitable zone is located closer to the star for M dwarfs than for Sun-like stars (e.g. Kasting et al. 1993; Kopparapu et al. 2013). This translates into orbital periods in the order of days to weeks and thus, combined with the low masses, to RV semi-amplitudes of $K \gtrsim 1 \mathrm{~m} \mathrm{~s}^{-1}$ (Endl et al. 2003). While high-resolution Echelle spectrographs such as HARPS (Mayor et al. 2003), HIRES (Vogt et al. 1994), and UVES (Dekker et al. 2000) reached the necessary precision for detecting RV modulations in that order of magnitude around twenty years ago, more recent instruments such as CRIRES+ (Dorn et al. 2014), HPF (Mahadevan et al. 2014), IRD (Kotani et al. 2014), NIRPS (Wildi et al. 2017), and SPIRou (Donati et al. 2018) aim to reach the same precision at nearinfrared wavelengths, where $\mathrm{M}$ dwarfs emit more light than at visible-light wavelengths. However, with the exception of spectral subtypes later than M7, the far-red spectral range between $7000 \AA$ and $9000 \AA$ allows for a higher RV precision than the near-infrared range covered by these spectrographs because it contains more spectral lines that can be used for measuring the Doppler shift (Artigau et al. 2018; Reiners et al. 2018b; Reiners \& Zechmeister 2020).

\subsubsection{CARMENES}

The Calar Alto high-Resolution search for $M$ dwarfs with Exo-earths with Near-infrared and optical Echelle Spectrographs (CARMENES, Quirrenbach et al. 2018) has been surveying a sample of more than 350 stars using the RV method since January 2016. With a beam splitter separating the light at $9600 \AA$ into the visible-light (VIS) and near-infrared (NIR) channels, CARMENES covers the wavelength range from $5200 \AA$ to $17100 \AA$ with the exception of several inter-order gaps at wavelengths $\lambda>11400 \AA$, as shown in Fig. 1.8. While the VIS channel spectrograph operates at room temperature, the NIR channel spectrograph is cooled to $140 \mathrm{~K}$ to avoid thermal contamination of the spectra. Both spectrograph channels provide high-resolution Echelle spectra with a resolving power of $R=94600$ in the VIS and $R=80400$ in the NIR channel. Each channel is fed by two fibers, one carrying the stellar light and the other used for simultaneously monitoring either sky emission close to the star or RV drifts using Fabry-Pérot etalons (Schäfer et al. 2018). Bauer et al. (2020) demonstrated that the VIS channel has a median RV precision of $1.2 \mathrm{~m} \mathrm{~s}^{-1}$, whereas the NIR channel reaches a median RV precision of $3.7 \mathrm{~m} \mathrm{~s}^{-1}$ using only the orders that contain most RV information and are least contaminated by telluric absorption bands.

CARMENES is mounted at the $3.5 \mathrm{~m}$ telescope of the Calar Alto Observatory in southern Spain. Both the size and the location of the telescope impose limits on which stars can reasonably be observed. In general, only stars that are well observable from the geographical latitude of Calar Alto $\left(37^{\circ} \mathrm{N}\right)$ are considered, so most stars in the CARMENES sample have a declination $\delta>-23^{\circ}$. To minimise the required exposure time for each spectrum, the brightest suitable stars should be observed. A pre-selection of 2190 late-type stars (as 


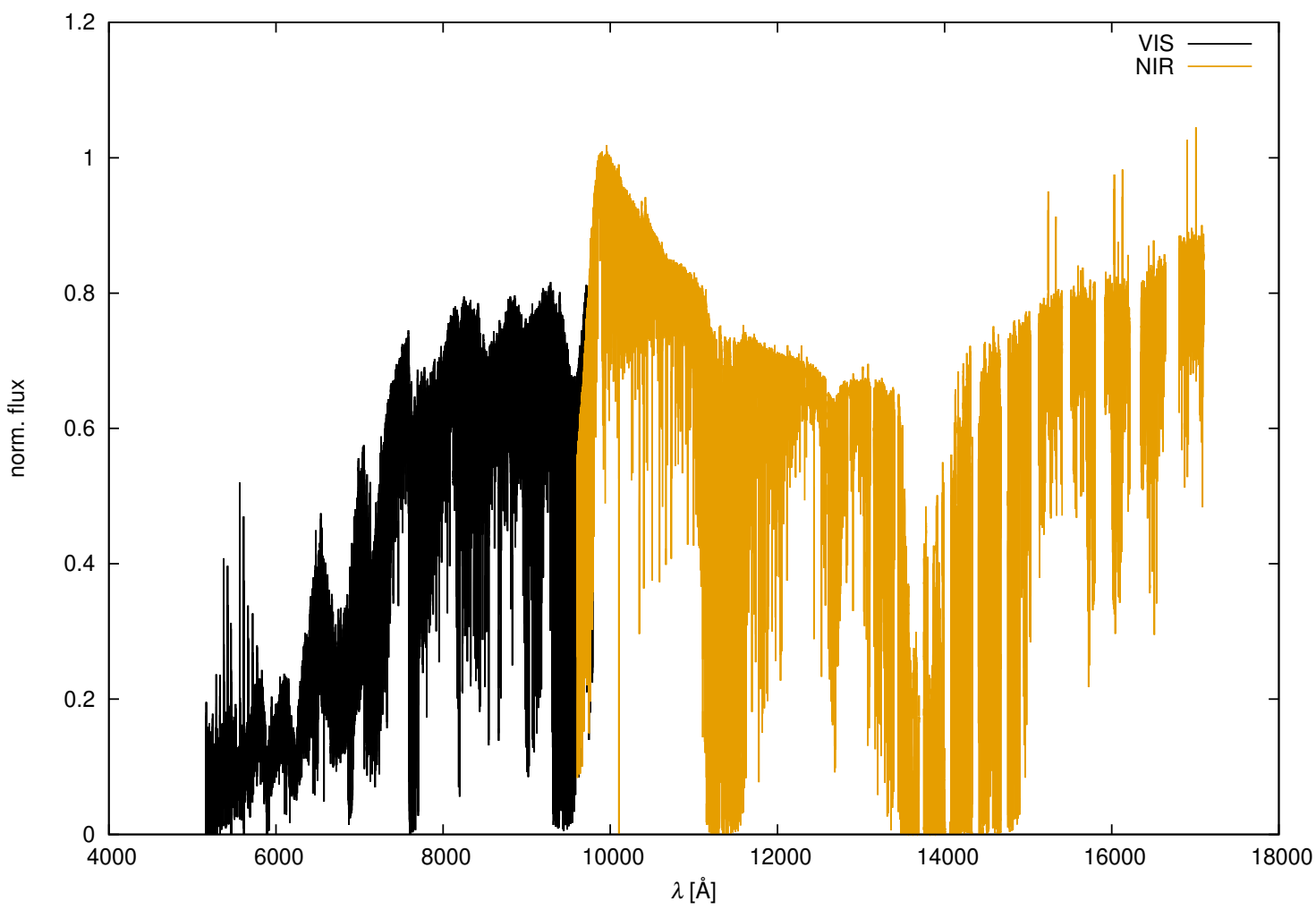

Figure 1.8.: One-dimensional spectrum extracted from the CARMENES spectrum of Luyten's star on 25 February 2016 at 21:07:51 UT shown in Fig. 1.1. The VIS channel spectrum is shown in black, the NIR channel spectrum is shown in orange. Inter-order gaps occur in the NIR channel at wavelengths $\lambda>11400 \AA$.
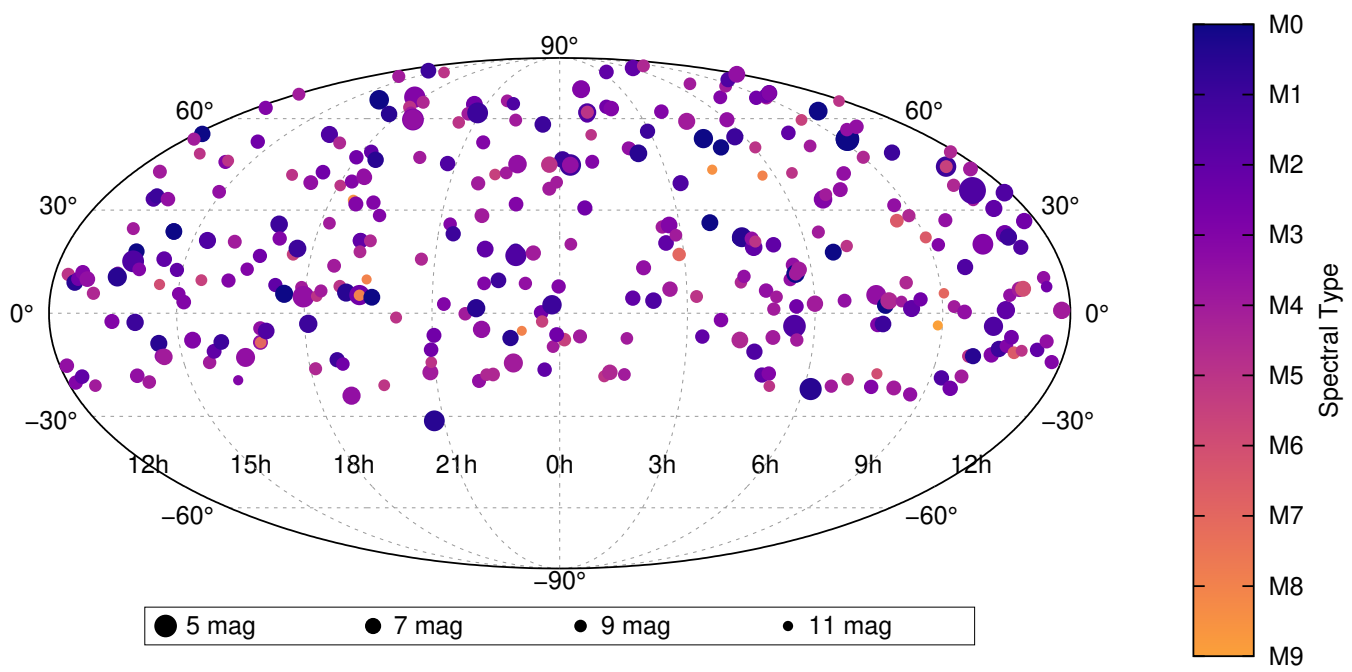

Figure 1.9.: Distribution of the 337 CARMENES sample stars analysed in this thesis on the sky. Colours correspond to spectral subtypes, circle size reflects the J-band magnitude. 
of 13 November 2019) with a spectral-type dependent J-band magnitude threshold between 8.5 mag for M0 and 11.5 mag for M6 and later was collected in the CARMEN(ES) Cool dwarf Information and daTa Archive (Carmencita, Caballero et al. 2016). From this input catalogue, more than 300 stars were selected for the CARMENES survey, mostly with a J-band magnitude brighter than $7 \mathrm{mag}$ for M0 and 11.5 mag for M9, and discarding stars with a known physical or optical companion within 5" (Reiners et al. 2018b). As M dwarfs of the same spectral type have similar luminosities, the brightness threshold effectively translates into a distance limit. While the distribution of the 337 CARMENES sample stars analysed in this thesis on the sky that is shown in Fig. 1.9 is close to uniform, the sample contains very few stars with a spectral type later than M5.

As of 15 October 2020, CARMENES has discovered 24 planets around M dwarfs including the first discovery based only on CARMENES data (HD 147379 b, Reiners et al. 2018a), a planet around the closest single star to Sun (Barnard's Star b, Ribas et al. 2018), two Earth-like planets around Teegarden's star (Zechmeister et al. 2019), a three-planet system with a transiting Earth-like planet (GJ 357 b, Luque et al. 2019b), and a giant planet that further contrains planet formation theories (GJ 3512 b, Morales et al. 2019). Another eight planets were found around earlier-type stars (Luque et al. 2019a; Palle et al. 2019; Hidalgo et al. 2020). In addition to finding planets, CARMENES has been used to derive stellar parameters (e.g. Passegger et al. 2018; Schweitzer et al. 2019), and to study exoplanet atmospheres (e.g. Allart et al. 2018; Nortmann et al. 2018; Alonso-Floriano et al. 2019; Yan et al. 2019) and stellar activity (e.g. Tal-Or et al. 2018; Fuhrmeister et al. 2018; Hintz et al. 2019; Schöfer et al. 2019).

\subsection{Stellar activity}

Stellar activity is an umbrella term for a plethora of variability phenomena in the stellar atmosphere that evolve on time scales from minutes to years, much shorter than the time scales of stellar evolution. Because these phenomena can be observed with detailed spatial resolution only for the Sun, the mostly disc-integrated observations of other stars are generally interpreted against the background of the solar phenomena. I therefore combine both the solar and the stellar perspective in the following overview of activity phenomena.

\subsubsection{Observed phenomena}

The first observed stellar activity phenomenon was the presence of dark spots on the Sun. As the largest sunspots can be visible without optical magnification if the light is sufficiently dimmed, the oldest recorded observations date back more than two millennia (e.g. Yau \& Stephenson 1988; Vaquero 2007). While first direct images of spots on other stars were obtained using interferometry in more recent years (Parks et al. 2015; Roettenbacher 

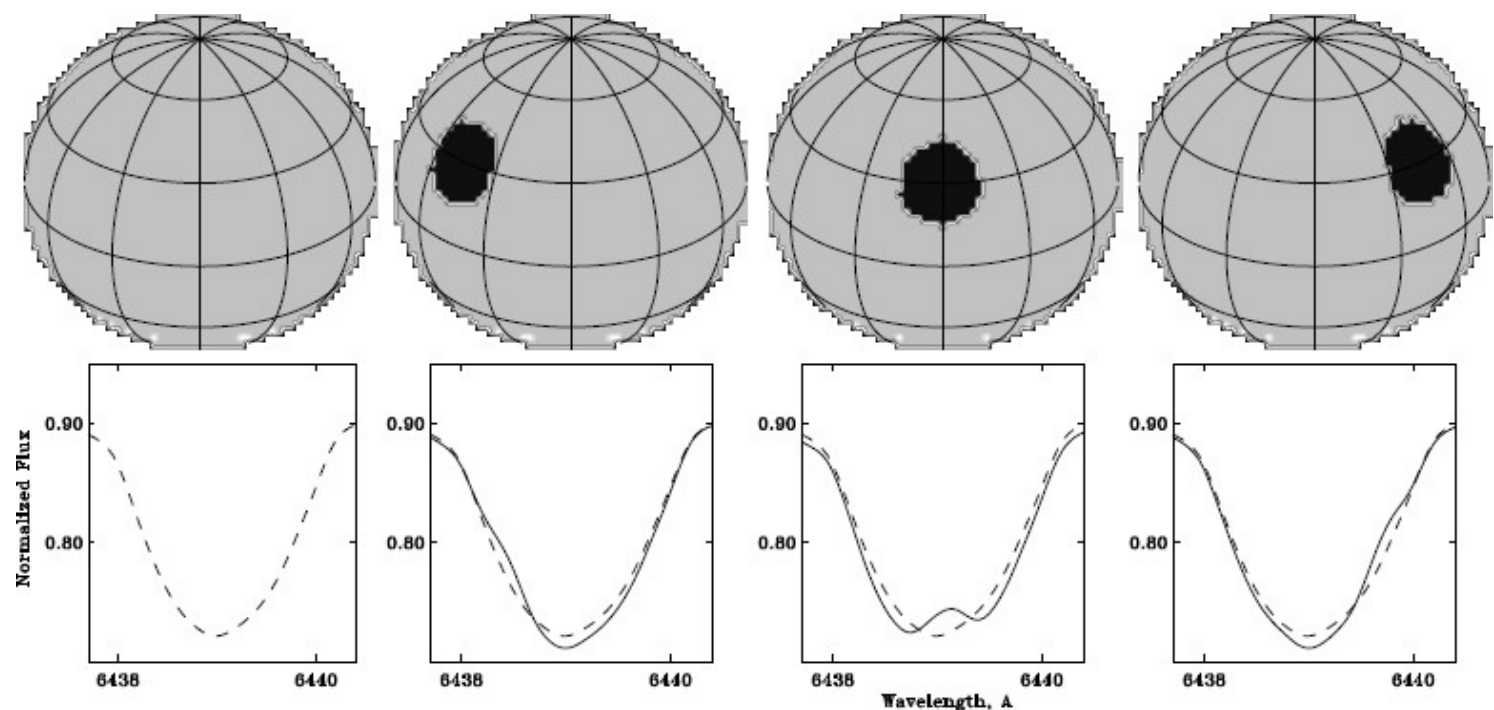

Figure 1.10.: A starspot on a fast rotating star moves across the hemisphere faced towards the observer as the star rotates, disturbing the observed flux at different Doppler shifts corresponding to the radial velocity of the spot with respect to the observer. This leads to a changing distortion in the observed spectral line profile (solid line) from the spectrum of the same star with no spots (dashed line). Image credit: Berdyugina (2005), used under CC-BY 4.0

et al. 2016), evidence for the existence of starspots was found before in photometric and spectroscopic data. As the star rotates, asymmetric spot distributions can lead to varying spot coverages of the hemisphere faced towards the observer and thus disc-integrated brightness variations. Kron (1947) proposed spots as an explanation for asymmetries and depth variations of the minima in the light curve of an eclipsing binary. Chugainov (1966) reported a periodic brightness variation for BY Dra, a star that did not show this behaviour in previous observations and was not known to be a binary system by then. While it was found to be a spectroscopic binary later, the orbital period did not match the photometric period and the observations were best explained as the star having spots and rotating with the photometric period (Bopp \& Evans 1973). Light curves of planet transits provide another photometric way to study starspots, as the planet blocks less light while it passes a spot (e.g. Silva 2003; Silva-Valio et al. 2010; Tregloan-Reed \& Unda-Sanzana 2019).

Starspots appear darker than the surrounding photosphere because they are cooler by hundreds of Kelvin for M dwarfs and up to $2000 \mathrm{~K}$ for Sun-like stars (Berdyugina 2005). As described in Sect. 1.2.1, presence and strength of spectral lines are temperaturedependent, so the spectrum of a cooler spot is different from the spectrum of the surroundings. Vogt (1979) first reported the presence of molecular absorption bands characteristic for $\mathrm{M}$ dwarfs in the spectrum of a spotted earlier-type star, and Huenemoerder et al. (1989) found a correlation between the strength of a TiO absorption band and the brightness of another earlier-type star. Neff et al. (1995) developed a method to derive the spot temperature and the fraction of the stellar disc covered by spots from molecular absorption bands, whereas Catalano et al. (2002) derived the same parameters from a set 
of atomic lines. Depending on its location on the stellar surface, a spot moves towards or away from the observer at different velocities as the star rotates. For fast rotating stars with sufficient rotational line broadening, this causes measurable a measurable distortion in the spectral line profiles at the position corresponding to the velocity of the spot as illustrated in Fig. 1.10. The Doppler Imaging technique by Vogt \& Penrod (1983) uses these distortions to create stellar surface maps and has been successfully used for various types of stars including M dwarfs (e.g. Barnes et al. 2002, 2015). Doppler Imaging maps revealed that many stars, unlike the Sun, have spots at high latitudes or even at the poles (e.g. Strassmeier et al. 1991; Kuerster et al. 1994; Jeffers et al. 2002), and that otherwise similar stars can have significantly different spot distributions (Barnes et al. 2017).

A complicating factor for indirect observations of starspots is that they are not constant, but can emerge and decay on time scales similar to the stellar rotation period. For the Sun, the lifetime of both individual and groups of spots is roughly proportional to their area (e.g. Petrovay \& van Driel-Gesztelyi 1997; Henwood et al. 2010). For other stars, individual spots in a group may not be resolved, but the observed spot lifetimes also increase with their area (e.g Hall \& Henry 1994; Namekata et al. 2019). As spots decay and new spots emerge, the total number of spots and fraction of the stellar surface covered by them vary. Schwabe (1844) and Wolf (1852) found a cycle of around 11 years in the number of sunspots, although Spörer (1887) and Maunder (1922) found evidence for an extended period with few sunspots in the second half of the seventeenth century. Long-term brightness variations reveal similar cycles for other stars (e.g. Strassmeier et al. 1997; Suárez Mascareño et al. 2016). Carrington (1858) first reported that sunspots appear predominantly at certain latitudes that change over the course of the cycle. Because the Sun does not rotate like a rigid body, but slower at higher latitudes than at the equator (differential rotation, e.g. Stix 1989), this has the consequence that a rotation period derived from disc-integrated solar brightness measurements also changes over the cycle. Evidence for changing active latitudes and differential rotation was also found in other stars (e.g. Donati \& Collier Cameron 1997; Reiners \& Schmitt 2003), with weaker shears at lower effective temperatures (e.g. Barnes et al. 2005; Reinhold et al. 2013). As shown by Basri \& Shah (2020), the impact of limited spot lifetimes on the light curve is larger than the impact of differential rotation, and both factors can lead to light curves with one dip per rotation period at some times, but two dips at other times. This behaviour is commonly observed in photometric surveys (e.g. Basri \& Nguyen 2018). For further information on starspots in general, I refer to the reviews by Berdyugina (2005) and Strassmeier (2009).

Contrary to intuition, the Sun appears brighter at the maximum of the sunspot cycle than at the minimum (e.g. Willson \& Hudson 1991). This is caused by smaller hot and thus bright faculae, which appear both in the vicinity of the cool, dark spots and in a network across the photosphere (e.g. Fröhlich 2002; Walton et al. 2003). While the solar luminosity over the course of a cycle is dominated by the facular contribution, spots can dominate the brightness variations of other stars. Radick et al. (1998) found that the brightness of more active stars decreases towards the maxima of their activity cycles and is hence spotdominated, whereas the brightness of less active stars, including the Sun, increases with increasing activity and is hence faculae-dominated. This pattern does not hold true for 


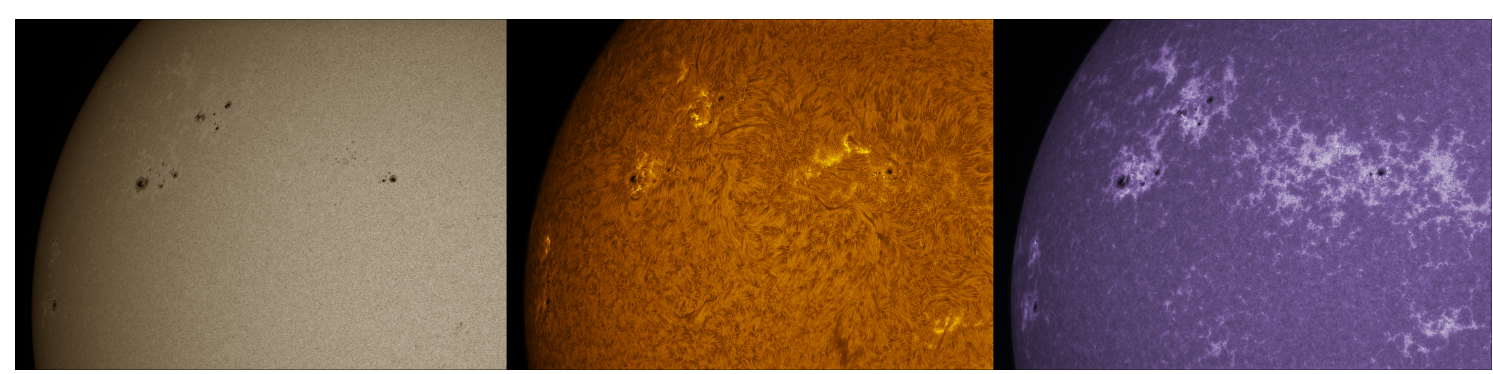

Figure 1.11.: Sector of the solar disc with sunspots, faculae, and plages in visible light (left) and narrowband $\mathrm{H} \alpha$ (centre) and Ca K (right) filters on 6 June 2015. Image credit: Pedro Ré6

brightness variations on the rotational time scale. As shown by Shapiro et al. (2016), the rotational solar brightness variability is spot-dominated, but would be faculae-dominated if observed from lower inclination angles. Based on the rotational modulation in the light curves of the M8 dwarf TRAPPIST-1, Morris et al. (2018) suggested the existence of bright spots that are larger and hotter than expected for faculae. Therefore, faculae may not be the only hot and bright stellar surface features.

Hale (1908) found Zeeman splitting of spectral lines from sunspots and thus related sunspots to magnetic fields. Magnetic fields also appear in the network of faculae outside of sunspots (e.g. Chapman \& Sheeley 1968). Preston (1971) first derived mean surface magnetic fields of other stars from Zeeman splitting. More advanced methods allow to quantify both the magnetic field strength and the fraction of the stellar disc covered by magnetic fields from Zeeman-broadened spectral lines (e.g. Robinson 1980; Saar 1988; Valenti \& Johns-Krull 2001; Shulyak et al. 2017). As found by Berdyugina \& Solanki (2002), the dominant molecular absorption bands in M dwarf spectra are magnetically sensitive and therefore useful to measure the magnetic fields of $M$ dwarfs or spots on earlier-type stars. By combining the Doppler Imaging technique with polarimetry, Zeeman Doppler Imaging reveals information on the geometry of the magnetic fields (Semel 1989; Donati et al. 1989). A series of papers by Donati et al. (2008), Morin et al. (2008), and Morin et al. (2010) presented Zeeman Doppler images for a sample of early-, mid-, and late-type $M$ dwarfs, revealing that early $M$ dwarfs have large-scale magnetic fields with strong toroidal and non-axisymmetric poloidal components similar to those observed on Sun-like stars, whereas on fully convective late M dwarfs, also fields with an axisymmetric poloidal components are found. In the case of the Sun, the polarity of the magnetic field flips with each 11-year sunspot cycle, resulting in a 22-year magnetic cycle (Weiss 1990). Boro Saikia et al. (2016) found evidence for a similar cycle in Zeeman Doppler images of a late K dwarf. The methods and results of stellar magnetic field measurements were reviewed by Reiners (2012) and Linsky \& Schöller (2015).

While the photosphere is the dominant layer of the stellar atmosphere in the visible-light spectral range, activity phenomena can also be observed in the outer layers. Young (1872) first observed the $\mathrm{Ca}$ II $\mathrm{H}$ and $\mathrm{K}$ Fraunhofer lines (hereafter $\mathrm{Ca} \mathrm{H \& K}$ ) to be in emission in

\footnotetext{
${ }^{6}$ https://re.apaaweb.com/sun_h_alpha_2015-06.html
} 
spectra of the solar chromosphere obtained at the limb of the solar disc and in spectra of regions close to sunspots. Narrowband observations of the Sun in $\mathrm{Ca} \mathrm{H}$ or K filters reveal that bright chromospheric plage regions mirror the bright photospheric network (e.g. Hall 2008), as can be seen in Fig. 1.11. In disc-integrated solar spectra, the intensity of the $\mathrm{Ca} \mathrm{H} \& \mathrm{~K}$ lines varies with the same 11-year cycle as the number of sunspots (Livingston et al. 2007). After Eberhard \& Schwarzschild (1913) found emissive cores in the Ca H\&K lines of Arcturus and other stars, these lines became a widely used proxy for stellar chromospheric activity, with surveys such as the Mount Wilson Observatory HK Project (e.g. Wilson 1968, 1978; Vaughan et al. 1978; Duncan et al. 1991; Baliunas et al. 1995; Boro Saikia et al. 2018) collecting data of thousands of stars over multiple decades. Similar to starspots causing brightness modulations with the rotation period, asymmetrically distributed plage regions can lead to measurable modulations of the Ca H\&K intensity (e.g. Vaughan et al. 1981; Donahue et al. 1996; Suárez Mascareño et al. 2015). Baliunas et al. (1995) found that some stars also have a chromospheric activity cycle similar to the Sun, whereas other stars show high average activity levels with no cyclic variation or low average activity levels with little variation.

For $\mathrm{M}$ dwarfs, the $\mathrm{Ca} \mathrm{H} \& \mathrm{~K}$ lines are difficult to observe because they are located at the blue edge of the visible-light range, where $\mathrm{M}$ dwarfs emit little flux. A more conveniently located and thus frequently used chromospheric activity indicator for $\mathrm{M}$ dwarfs is the hydrogen Balmer- $\alpha$ line (H $\alpha$; e.g. Stauffer \& Hartmann 1986; West et al. 2004; Jeffers et al. 2018). It is this spectral feature that causes the solar chromosphere to appear as a thin red line at the solar limb during a total eclipse and led to the name chromosphere for that atmospheric layer. As M dwarfs show $\mathrm{H} \alpha$ either in emission or in absorption, those with $\mathrm{H} \alpha$ in emission are also called dMe stars (Joy 1947) or active M dwarfs (e.g. Young et al. 1989). However, even $\mathrm{M}$ dwarfs with $\mathrm{H} \alpha$ in absorption can be active in the sense of showing emission in Ca H\&K (e.g. Joy \& Abt 1974). To avoid this ambiguity, I will use the terms $H \alpha$ active and $H \alpha$ inactive for $\mathrm{M}$ dwarfs with and without significant emission in $\mathrm{H} \alpha$, respectively, throughout this thesis. Chromospheric models by Cram \& Mullan (1979) and Cram \& Giampapa (1987) showed that while the core emission in Ca H\&K increases monotonically with increasing chromospheric temperature and density, $\mathrm{H} \alpha$ first appears as an increasingly deep absorption line, then starts to show emission in the line wings and a shallower core, and finally becomes an increasingly strong emission line with a central reversal. Houdebine (2012) found observational evidence for a non-linear correlation between the strengths of the $\mathrm{H} \alpha$ and $\mathrm{Ca} \mathrm{K}$ lines in sets of M1 dwarfs and, less clear, M4 dwarfs. Therefore, $\mathrm{H} \alpha$ is no conclusive activity indicator at low chromospheric activity levels, i.e. for $\mathrm{H} \alpha$ inactive $\mathrm{M}$ dwarfs.

A number of further spectral features has been explored as chromospheric activity indicators. Among these features is the Na I D doublet (hereafter Na D, e.g. Andretta et al. 1997; Díaz et al. 2007; Gomes da Silva et al. 2011), which is located in the bluest part of the spectral range covered by CARMENES. A nearby helium line, often called $\mathrm{He}$ i $\mathrm{D}_{3}$ (hereafter $\mathrm{He} \mathrm{D}_{3}$ ), usually appears in emission when emissive line cores in $\mathrm{Na} \mathrm{D}$ are observed (Houdebine et al. 2009) and is correlated with $\mathrm{H} \alpha$ (Gizis et al. 2002). In the far-red spectral range, the $\mathrm{K}$ I $\lambda 7700 \AA$ and $\mathrm{Na}$ I $\lambda 8200 \AA$ doublets were found to become stronger 
absorption lines with increasing $\mathrm{H} \alpha$ emission for late-type members of the Praesepe cluster by Kafka \& Honeycutt (2006), whereas Robertson et al. (2016) found the opposite for Proxima Centauri. The Ca II infrared triplet (hereafter Ca IRT) lines correspond to transitions between the same upper energy state of the Calcium ion as $\mathrm{Ca} H \& \mathrm{~K}$ and a different lower energy state (e.g. Linsky \& Avrett 1970). As a consequence, the Ca IRT lines indicate the chromospheric activity level in a similar way to $\mathrm{Ca} H \& \mathrm{~K}$, as was observed both in spectra of different regions on the solar disc (e.g. Shine \& Linsky 1972) and in stellar surveys (e.g. Linsky et al. 1979; Busà et al. 2007; Martínez-Arnáiz et al. 2011; Martin et al. 2017). Models by Andretta et al. (1997) and Short \& Doyle (1997) suggested that the lines of the hydrogen Paschen $(\mathrm{Pa})$ series at near-infrared wavelengths are sensitive to the chromospheric conditions in active M dwarfs. Short \& Doyle (1998) and Houdebine et al. (2009) found examples of $\mathrm{Pa} \beta$ and $\mathrm{Pa} \epsilon$ appearing as absorption lines. Another chromospheric line in the near-infrared range is the He I $\lambda 10833 \AA$ (hereafter He 10833) line (e.g. Vaughan \& Zirin 1968; Zirin 1982; Sanz-Forcada \& Dupree 2008; Fuhrmeister et al. 2019a). The spectral regions around the chromospheric lines analysed in this thesis are shown in Fig. 2.2. A more complete list of chromospheric activity indicators including the blue and ultraviolet (UV) spectral range not accessible with CARMENES, and a discussion of their formation mechanisms can be found in a review by Linsky (2017). While all chromospheric activity indicators suggest that chromospheres are hotter and less dense than the underlying photospheres, each indicator is most sensitive in a different temperature and density range and can thus be attributed to a certain region within the chromosphere (e.g. Vernazza et al. 1981; Mauas 2000). Different indicators can therefore track different active regions, as is illustrated by the different appearances of the Sun in $\mathrm{H} \alpha$ and $\mathrm{Ca} \mathrm{K}$ in Fig. 1.11.

Occasionally, the spectral lines sensitive to chromospheric activity can be observed in significantly enhanced emission. An example of such a flare in $\mathrm{H} \alpha$ is shown in Fig. 1.12: The $\mathrm{H} \alpha$ emission line in the spectrum of the active M3.5 dwarf EV Lac appeared very strong on 1 September 2016 before going back to its quiescent strength in the next days. While the first stellar flare was observed in chromospheric hydrogen and helium lines in the blue part of the visible-light range (Joy \& Humason 1949), flares affect all chromospheric activity indicators and the continuum flux across the spectrum (e.g. Liebert et al. 1999; Fuhrmeister et al. 2008; Schmidt et al. 2012). The enhanced continuum flux can also be detected photometrically, not only at visible-light wavelengths (e.g. Kunkel 1970; Hawley \& Pettersen 1991; Schmidt et al. 2019), but from X-rays to radio emission (e.g. Haisch et al. 1981; Osten et al. 2005; Smith et al. 2005). Flares have been reported for various types of stars (e.g. Pettersen 1989; Linsky 2000) and are increasingly common in latertype dwarfs (e.g. Davenport 2016; Yang et al. 2017). Observations of solar flares date back to Carrington (1859) and Hodgson (1859, incidentally, 157 years to the day prior to the EV Lac flare in Fig. 1.12), and revealed that they are localised events and occur preferably in regions with complex magnetic fields (e.g. Benz 2017). As these regions are associated with sunspots, the flaring rate of the Sun is strongly correlated with the 11-year sunspot cycle (Hathaway 2015). Similarly, more flares are observed from $\mathrm{H} \alpha$ active $\mathrm{M}$ dwarfs than from $\mathrm{H} \alpha$ inactive M dwarfs (e.g. Kowalski et al. 2009). Gershberg (1972) and 


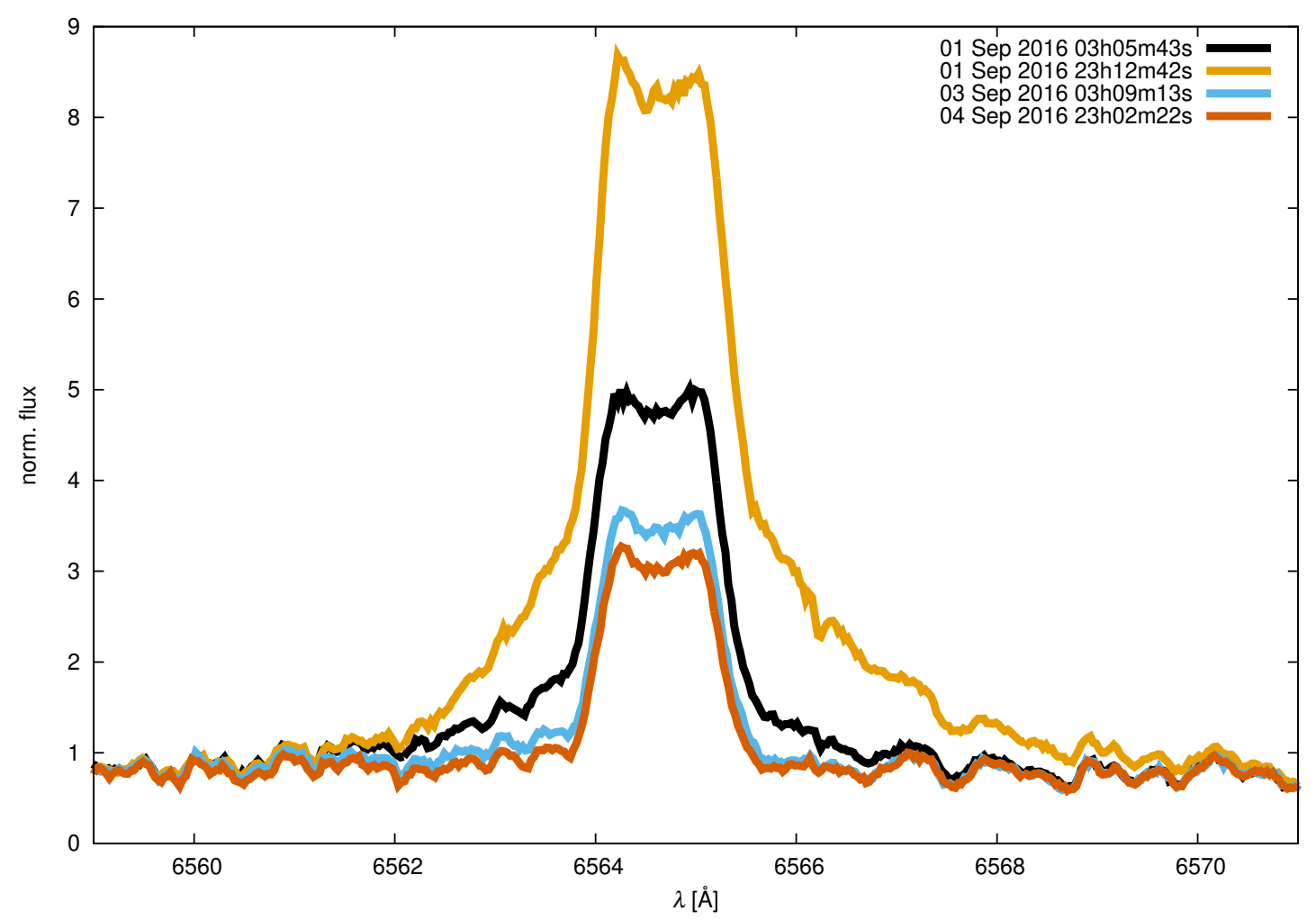

Figure 1.12.: $\mathrm{H} \alpha$ line in spectra of EV Lac. The emission increases significantly during flares on 1 September 2016 (black, orange) in comparison to the quiescent strength in the following days (blue, red).

Lacy et al. (1976) first found that stronger flares occur less frequently following a power law in a sample of $\mathrm{M}$ dwarfs. Similar power-law relations with different exponents were found for other stars, including the Sun (e.g. Schrijver et al. 2012). Stronger flares decay slower (e.g. Pettersen 1989), with the strongest flares being detectable for several hours (Kowalski et al. 2010). As can also be seen in Fig. 1.12, the enhancement of emission lines during a flare is not necessarily symmetric around the line cores. Line asymmetries are especially prominent in $\mathrm{H} \alpha$ (Fuhrmeister et al. 2018) and can be attributed to a Doppler shift caused by the emitting material rising in the chromosphere at the onset of the flare (blue asymmetry) or moving down again during the decay (red asymmetry; Ichimoto \& Kurokawa 1984; Cho et al. 2016; Johnson et al. 2021). Flares were discussed in detail in the reviews by Haisch et al. (1991) and Benz \& Güdel (2010).

Above the chromosphere lies the corona. As the solar corona becomes visible during total eclipses, it has been observed for millennia, and in the late nineteenth century, Jules Janssen noticed that the shape of the solar corona varies with the sunspot cycle (Littmann et al. 2008). The detection of highly ionised and forbidden metal lines in solar corona spectra lead to the assumption that the corona consists of hot, low-density plasma (Edlén 1943). As a consequence, stellar coronae are best observed at extreme UV and X-ray wavelengths, while the photosphere outshines the corona at longer wavelengths such as 
the visible-light and near-infrared range covered by CARMENES. Because X-rays are absorbed by the Earth's atmosphere, the first detections of X-rays from the Sun (Burnight 1949) and another star (Capella, Catura et al. 1975) were obtained during rocket flights. X-ray space observatories such as ROSAT, Chandra, and XMM-Newton revealed that Xray emission similar to the solar corona is common among Sun-like and later-type stars, as outlined in the reviews by Güdel (2004) and Güdel \& Nazé (2009). A second window into stellar coronae is provided by nonthermal radio emission, as detailed in a review by Güdel (2002). Güdel \& Benz (1993) found a very strong correlation between X-ray and radio emission for a variety of active stars, which also holds true for solar flares (Benz \& Güdel 1994). Similar correlations were found between coronal X-ray emission and chromospheric activity indicators (e.g. Ayres et al. 1981; Schrijver 1987).

In addition to electromagnetic radiation, the high temperature and the resulting high kinetic energy allow plasma particles to escape the corona. Parker (1958) first presented a model of a continuous solar wind of escaping coronal plasma motivated by the suggestion of Biermann (1951) that a plasma stream can explain the observed shapes of comet tails. While the solar wind was first directly observed with the Luna spacecrafts (Gringauz et al. 1962), the clearest evidence for stellar winds of Sun-like and and later-type stars is provided indirectly by absorption in the UV hydrogen Lyman- $\alpha$ line as reviewed by Wood (2004). Wood et al. (2005) found that more active stars have stronger stellar winds, but this relation breaks down for the most active stars. A short-term but more violent coronal plasma release phenomenon are coronal mass ejections (CMEs). From the Sun, CMEs have been observed for 50 years since the first report by Tousey (1973) and occur often, but not always, together with flares (e.g. Kahler 1992; Yashiro et al. 2005). As with flares, the occurrence rate of solar CMEs strongly follows the 11-year sunspot cycle (e.g. Webb \& Howard 1994; Lamy et al. 2019). Webb \& Howard (2012) and Chen (2017) reviewed observations and theoretical models of solar CMEs. On other stars, CMEs may be detected indirectly through X-ray absorption by the ejected material at the onset of large flares (e.g. Favata \& Schmitt 1999; Moschou et al. 2017) or strong blue asymmetries of emission lines (e.g. Houdebine et al. 1990; Guenther \& Emerson 1997; Bond et al. 2001; Vida et al. 2016). Moschou et al. (2019) compiled a list of all stellar CME candidates that contains only 15 stars. However, blue asymmetries that correspond to material velocities below the escape velocity of the stars are observed more commonly and may still be signatures of CMEs because the Doppler shift measures only the line-of-sight component of the velocity (e.g. Leitzinger et al. 2011; Vida et al. 2019; Muheki et al. 2020). An alternative way to detect CMEs is provided by low-frequency radio bursts that are often generated by solar CMEs (e.g. Gopalswamy et al. 2009). For M dwarfs, however, efforts to observe the same type of radio bursts have remained unsuccessful so far (e.g. Crosley \& Osten 2018; Villadsen \& Hallinan 2019) and models arrive at different conclusions whether they can be generated (Mullan \& Paudel 2019; Alvarado-Gómez et al. 2020). 


\subsubsection{Origin of stellar activity}

The local activity phenomena described in the previous section often occur together, not only in the same atmospheric layer, but also across different layers. Global activity indicators for different atmospheric layers also show correlations. Therefore, it is plausible that all stellar activity phenomena can be traced back to one or more common sources. A first hint towards a possible source are the magnetic fields measured in solar spots and faculae. Stronger magnetic fields are measured on stars with stronger chromospheric and coronal emission (e.g. Pevtsov et al. 2003; Reiners et al. 2009; Reiners \& Basri 2010; Vidotto et al. 2014; Marsden et al. 2014). Because a direct heat flow from the cooler photosphere to the hotter outer atmospheric layers is ruled out by the second law of thermodynamics, other heating mechanisms are required to explain the hot temperatures in the chromosphere and corona that lead to the observed emission. First ideas suggested energy transport through acoustic waves (Biermann 1946; Schwarzschild 1948; Schatzman 1949) or magnetohydrodynamic waves (Alfvén 1947). In complementary theories, magnetic fields are dissipated to heat (e.g. Heyvaerts 1990). Parker (1988) proposed that the corona is heated by magnetic reconnections similar to flares, but on smaller scales (nanoflares). On the Sun, smaller flares are less often accompanied by CMEs than larger flares, supporting the theory that the energy released in nanoflares contributes to heating the corona (Yashiro et al. 2006). Schrijver (1987) found that the chromospheric emission has a basal component that is not correlated with coronal emission and decreases towards later spectral types, and suggested that this component is caused by acoustic wave heating. However, Judge et al. (2003) found evidence that weak magnetic fields cause the basal chromospheric heating, whereas Jefferies et al. (2006) proposed a combined magnetoacoustic heating mechanism. I refer to the reviews by Narain \& Ulmschneider $(1990,1996)$ for details on the various proposed heating mechanisms in outer stellar atmospheres.

Narain \& Ulmschneider (1996) conclude that magnetic mechanisms dominate the heating of chromospheres and coronae. This leads to the question of how the required magnetic fields are generated. As shown for a sample of M dwarfs in the left panel of Fig. 1.13, stars with short rotation periods are in general more active in terms of $\mathrm{H} \alpha$ emission than slow-rotating stars, with a saturation at the shortest rotation periods. This rotation-activity relation was found for various samples of Sun-like and later-type stars not only using chromospheric $\mathrm{H} \alpha$ emission as activity indicator (e.g. Soderblom et al. 1993; Delfosse et al. 1998; Reiners et al. 2012; Newton et al. 2017; Jeffers et al. 2018), but also using Ca H\&K emission (e.g. Catalano \& Marilli 1983; Noyes et al. 1984; Astudillo-Defru et al. 2017), or coronal X-ray or radio emission (e.g. Pallavicini et al. 1981; Stewart et al. 1988; Pizzolato et al. 2003; Wright et al. 2011; McLean et al. 2012). While some of the referenced studies considered the Rossby number Ro $=P_{\text {rot }} / \tau$ with the convective overturn time $\tau$ instead of the rotation period $P_{\text {rot }}$, the scatter around the relation can be minimised with a formulation using only $P_{\text {rot }}$ (Reiners et al. 2014). An interpretation of the relation between rotation and activity is that the kinetic energy of the rotating and convecting plasma in the stellar interior is converted to magnetic energy by dynamo processes. In most widelyaccepted models, the solar dynamo is driven by the alternation between a poloidal and 

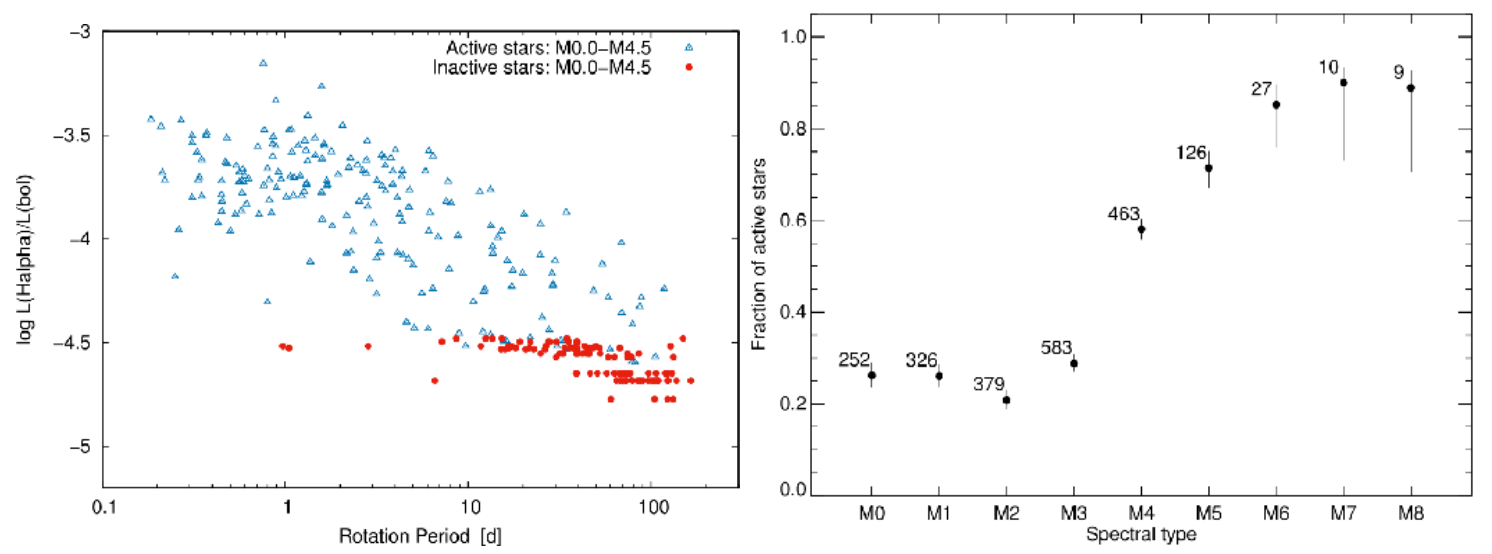

Figure 1.13.: Left: Normalised $\mathrm{H} \alpha$ luminosity as a function of the rotation period for stars in Carmencita. Right: Fraction of $\mathrm{H} \alpha$ active stars per spectral subtype. Numbers reflect the total number of stars of each subtype. Image credit: Jeffers et al. (2018), reproduced with permission, (c) ESO

a toroidal magnetic field over the course of the solar cycle. As demonstrated by Parker (1955), the shear from differential rotation generates the toroidal field component from the poloidal component ( $\Omega$ effect), and the poloidal component can be regenerated from the toroidal field by cyclonic convection ( $\alpha$ effect). In an alternative suggestion by Babcock (1961) and Leighton $(1964,1969)$, active regions drive the regeneration of the poloidal field instead of convection. Detailed discussions of solar dynamo models can be found in the reviews by Ossendrijver (2003) and Charbonneau (2020).

Models commonly place the solar dynamo in the tachocline between the radiative core and the convection zone. While later-type dwarfs down to early $M$ dwarfs have a similar internal structure and thus can host a Sun-like dynamo, M dwarfs of spectral subtypes later than M4 are fully convective and thus have no tachocline, as described in Sect. 1.2.4. However, the fraction of active stars increases at these spectral subtypes (e.g. West et al. 2004, 2015; Reiners et al. 2012; Jeffers et al. 2018), as shown in the right panel of Fig. 1.13, indicating the existence of a dynamo process within the convection zone. Durney et al. (1993) found that turbulent small-scale magnetic fields can be generated in the convection zone and suggested that fully convective stars thus have only small-scale turbulent dynamos. However, more recent models showed that fully convective stars can also generate large-scale magnetic fields (Chabrier \& Küker 2006; Dobler et al. 2006; Browning 2008). While the exact dynamo mechanisms at work remain unclear, Spruit (2011) suggested that Sun-like dynamos operate in the convection zone and not in the tachocline. Therefore, the fully convective stars may also have Sun-like dynamos. This is supported by the fact that the same rotation-activity relation is observed for both early and late M dwarfs (Wright \& Drake 2016; Wright et al. 2018).

Wilson (1963) found that stars in young clusters show stronger emission in Ca H\&K than old field stars of similar spectral types, thereby relating stellar activity and age. This relation was first quantified by Skumanich (1972), who found that both Ca H\&K emission 
and rotational velocity decay with the inverse square root of the age. A power-law decay with age is also observed for coronal X-ray and chromospheric UV emission, with a steeper decay at shorter wavelengths (e.g. Ribas et al. 2005; Stelzer et al. 2013). The declining activity is a consequence of the star spinning down because the stellar wind leads to a loss not only in mass, but also in angular momentum (e.g. Weber \& Davis 1967).

In binary systems, both tidal effects (e.g. Schrijver \& Zwaan 1991) and interaction of the stellar magnetic fields (e.g. Siarkowski et al. 1996) can affect the activity of each companion. Cuntz et al. (2000) suggested that close-by giant planets may strengthen the activity of their host stars in the same ways. Shkolnik et al. (2005) found two stars that showed periodic modulations of the $\mathrm{Ca} H \& \mathrm{~K}$ lines with the orbital periods of their planets instead of their rotation periods. However, this result could not be confirmed in later observations (Shkolnik et al. 2008). Additional observational hints at star-planet interactions were reviewed by Poppenhaeger (2019).

\subsubsection{Implications for exoplanet surveys}

The effects of stellar activity are an important factor for the detectability of exoplanets. In the context of RV surveys, stellar activity can introduce RV jitter in the same order of magnitude as the periodic modulation caused by an Earth-like exoplanet, and may therefore lead to false or controversial planet detections (e.g. Dumusque et al. 2012; Hatzes 2013; Anglada-Escudé et al. 2014, 2016; Robertson et al. 2015). On short time scales from minutes to days, one source of RV jitter is a blueshift caused by rising material in convection cells covering a larger fraction of the stellar surface than sinking material on the edges of the convection cells (e.g. Beckers \& Nelson 1978; Dravins 1982). While the impact of variations of this convective blueshift on RV measurements can be mitigated by using multiple observations per night (Dumusque et al. 2011), stellar activity increases these variations on longer time scales, as convection is suppressed by magnetic fields (e.g. Lagrange et al. 2010; Meunier et al. 2010; Jeffers et al. 2014; Bauer et al. 2018). In addition, line profile distortions introduced by active regions as shown in Fig. 1.10 can affect the RV measurements (e.g. Saar \& Donahue 1997; Queloz et al. 2001). Depending on the distribution and the lifetime of the active regions, this can lead to RV modulations with periods similar to the rotation period or its integer fractions (e.g. Desort et al. 2007; Hatzes 2013). On long time scales of several years, a magnetic cycle can induce an additional RV modulation (e.g. Lindegren \& Dravins 2003; Lovis et al. 2011; Delisle et al. 2018). While statistical methods (e.g. Tuomi et al. 2013; Haywood et al. 2014) or excluding activitysensitive lines from the RV measurement (Dumusque 2018) can help to mitigate activity effects on the measured RV, monitoring activity indicators remains important to improve these methods and avoid false detections.

Furthermore, stellar activity has to be considered in assessing the habitability of exoplanets. Not only may close-by planets affect the activity of their host stars as discussed in 
the last paragraph of the previous section, but an active star can also significantly influence the atmospheres of its planets. Stellar winds and CMEs can lead to a significant atmospheric mass loss, particularly if the planet lacks a shielding magnetic field (e.g. Wood 2006; Khodachenko et al. 2007; Zendejas et al. 2010; Cohen et al. 2014; Kay et al. 2016). Comparing the time scales of stellar evolution, atmosphere erosion by stellar winds, and life evolution, Lingam \& Loeb (2017a) found that early-type M dwarfs with masses around $0.55 M_{\odot}$ may host the planets with the largest biological diversity. Strong X-ray and extreme UV radiation from the corona heats the atmosphere and thus contributes to the erosion of the atmosphere (e.g. Vidal-Madjar et al. 2003; Lammer et al. 2003; Sanz-Forcada et al. 2011), but the remaining atmosphere after the evaporation of excess hydrogen and helium may be more life-friendly (e.g. Luger et al. 2015; Owen \& Mohanty 2016). Chromospheric UV emission can change the atmospheric composition by dissociating molecules such as carbon dioxide, ozone, and water (e.g. Barnes \& Heller 2013; Tian et al. 2014; Luger \& Barnes 2015). As shown by Segura et al. (2010), the increased short-wavelength radiation during typical $\mathrm{M}$ dwarf flares is effectively shielded by an ozone layer and may therefore not affect the habitability of a planet, unless the planet is hit by accompanying CMEs. The strongest flares may drive mass extiction events (Lingam \& Loeb 2017b), but the additional UV radiation may also enable the synthesis of biochemical compounds required for life (Toupance et al. 1977). Overall, less active stars with masses from the early-type $\mathrm{M}$ dwarf range up to solar mass stars may provide a more life-friendly environment on planets in their liquid water habitable zone than more active very late-type stars, and are therefore more promising targets for follow-up studies on planetary atmospheres and signs of extraterrestrial life. However, the minimum and maximum levels of stellar activity necessary to create and sustain life-friendly atmospheres are unclear.

\subsubsection{Outline}

This thesis uses $\mathrm{M}$ dwarf spectra obtained with CARMENES to derive indicators of their activity and investigates the correlations and temporal variation of these indicators. Because CARMENES covers the visible-light and near-infrared range, the spectra contain mainly information about the photospheres and several spectral features formed in the chromospheres of the studied stars, while their coronae are beyond the scope of this thesis.

I explain the data and the methods I used to derive activity indicators in Chapter 2. Chapters 3 and 4 present and analyse the obtained chromospheric and photospheric indicators, respectively, whereas Chapter 5 focuses on the modulation of the indicators with stellar rotation. Each of these chapters includes a discussion of the presented results. Chapter 6 concludes the thesis with a summary of the results and suggestions for further work. 



\section{Data and methods}

This chapter introduces the input data and the methods I used to derive the two sets of spectral indicators that are presented and analysed in this thesis. I describe the used set of CARMENES spectra in Sect. 2.1, and explain how I measured the strengths of atomic lines with a component generated in the chromosphere using a spectral subtraction technique in Sect. 2.2, and of molecular absorption bands formed in the photosphere in Sect. 2.3.

\subsection{CARMENES spectra}

The CARMENES survey started in January 2016 and is still ongoing as of December 2020. For this thesis, only spectra recorded until 8 November 2019 were considered. Until that date, 354 stars were observed in total 16684 times with the VIS channel and 16142 times with the NIR channel during Guaranteed Time Observations. Of these 354 stars, I excluded nine double-lined spectroscopic binaries discovered by Baroch et al. (2018) from my analysis because the activity indicators of the two binary components cannot be measured separately using my methods. However, combined indicators of both components might not include the full activity signal if measured in the same spectral range as used for single stars. I also excluded five $\mathrm{K}$ dwarfs, as they are a separate group of objects. A further three stars were observed only once or twice. Therefore, the temporal variation of their activity indicators could not be analysed. I list the remaining 337 stars in Appendix A. Not all spectra of these stars yielded successful measurements. In total, 15868 VIS spectra and 15351 NIR spectra could be successfully analysed for this thesis. As the histogram in Fig. 2.1 shows, these spectra are very unevenly distributed among the target stars. While less than 10 VIS spectra could be analysed for 56 stars and less than 10 NIR spectra could be analysed for 62 stars, there are 10 stars with more than 200 analysed spectra from each channel. For both channels, the median is 23 spectra per star. The colour-coding by spectral subtype reveals that earlier-type $\mathrm{M}$ dwarfs constitute a larger fraction of the heavily observed stars than of the stars with fewer observations.

All spectra were reduced by Dr. Mathias Zechmeister (Institut für Astrophysik Göttingen) and Dr. Florian Bauer (Instituto de Astrofísica de Andalucía, Granada, Spain) using CARACAL v2.20. The CARACAL pipeline is based on the IDL REDUCE package (Piskunov \& Valenti 2002) and implements standard data reduction steps, the flat-relative 


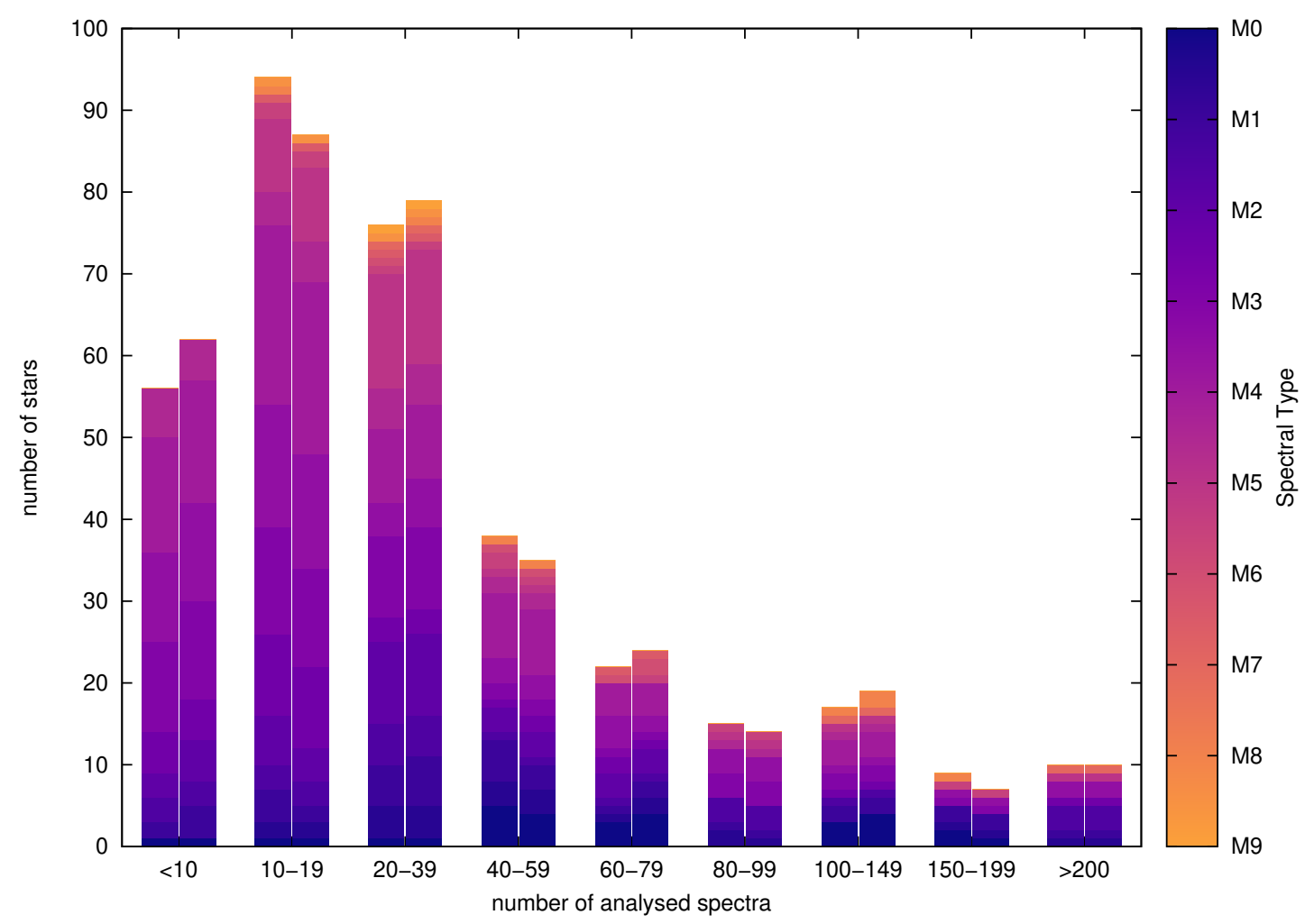

Figure 2.1.: Number of stars with a given number of analysed spectra, colour-coded by spectral subtype. For each bin, the left bar corresponds to the VIS channel and the right bar corresponds to the NIR channel.

optimal extraction (FOX, Zechmeister et al. 2014), and a wavelength calibration using Fabry-Pérot etalons (Bauer et al. 2015).

In addition to the reduced spectra from individual observations, I also used co-added template spectra created by Dr. Mathias Zechmeister from all individual spectra of each star for each spectrograph channel using SERVAL (Zechmeister et al. 2018). These template spectra have two advantages over the individual spectra: The signal-to-noise ratio (SNR) is higher and telluric lines are mitigated because the co-adding procedure excludes data points outlying by more than $5 \sigma$. The telluric lines are strong outliers, because different barycentric velocities cause them to appear at different positions in the stellar spectrum at different observation times. I therefore used the co-added template spectra to measure the typical value of each of my spectral indicators for each star instead of averaging the values derived from the individual spectra. As spectra recorded during strong flaring events can also cause strong outliers that are excluded by the co-adding procedure, the values derived from the template spectra may be more representative of the usual activity level of the star than the average of individual spectra. However, I still used the individual spectra for investigating the temporal variations of the spectral indicators. 


\subsection{Strengths of chromospheric lines}

As $\mathrm{Ca} H \& \mathrm{~K}$ are not covered by CARMENES, $\mathrm{H} \alpha$ is the most established chromospheric activity indicator that can be measured in the scope of this thesis. Of the other spectral lines with a chromospheric component mentioned in Sect. 1.4.1, I consider the $\mathrm{He} \mathrm{D}_{3}$ line, the Na D doublet, and the Ca IRT in the spectral range of the VIS channel, and the He 10833 and $\mathrm{Pa} \beta$ lines in the NIR channel. The regions around these lines in selected spectra are shown in Fig. 2.2. I discard the K I $\lambda 7700 \AA$ and $\mathrm{Na}$ I $\lambda 8200 \AA$ doublets because they are heavily contaminated by telluric lines, and the higher order lines of the Paschen series because they are weaker than $\mathrm{Pa} \beta$. The $\mathrm{Pa} \alpha$ line at $18756.4 \AA$ is outside of the spectral coverage.

A common measure of the strength of a spectral line is the equivalent width (EW). It is defined as the width of a hypothetical rectangular complete absorption feature that covers the same area as the spectral line in a spectrum normalised to the surrounding continuum. This allows to compare the strength of spectral lines with different shapes. Emission lines can be described as negative absorption lines and therefore have negative EWs. In $\mathrm{M}$ dwarf spectra, a true continuum is hard to find. Therefore, the spectrum is usually normalised to a pseudo-continuum (PC), and the EW measured with respect to the PC is called a pseudo-equivalent width ( $\mathrm{pEW}$ ). In this thesis, I measure the pseudo-equivalent width after subtracting the spectrum of an inactive reference star, similar to the spectral subtraction technique used by Young et al. (1989) and Montes et al. (1995). Inspired by the $R_{\mathrm{HK}}^{\prime}$ index of the Ca H\&K lines (Noyes et al. 1984), where the prime denotes the subtraction of the photospheric components, I refer to the pseudo-equivalent width measured after the spectral subtraction as $\mathrm{pEW}^{\prime}$.

The steps for measuring the $\mathrm{pEW}^{\prime}$ are as follows: First, an artificial rotational broadening was applied to the co-added template spectrum of the reference star, if the investigated star has a measured projected rotational velocity according to Carmencita as listed in Table A.1. Next, the investigated spectrum was transformed to the same wavelength grid as the reference star spectrum. The Doppler shift between the spectra was determined from a Gaussian fit to the highest peak of the cross-correlation function of the spectral order containing the considered spectral line. Both the reference star spectrum and the investigated spectrum were then normalised to the mean flux in the respective PC ranges $\lambda_{1 / \mathrm{r}} \pm \Delta \lambda_{\mathrm{PC}}$ as listed in Table 2.1. The PC was then defined as a linear interpolation between the $\mathrm{PC}$ ranges in the normalised reference star spectrum:

$$
\mathrm{PC}(\lambda)=\mathrm{PC}_{1}+\frac{\mathrm{PC}_{\mathrm{r}}-\mathrm{PC}_{1}}{\lambda_{\mathrm{r}}-\lambda_{1}}\left(\lambda-\lambda_{1}\right)
$$

where $\mathrm{PC}_{1 / \mathrm{r}}$ are the 90th percentile of the flux in the PC ranges $\lambda_{1 / \mathrm{r}} \pm \Delta \lambda_{\mathrm{PC}}$ in the reference star spectrum. The choice of the 90th percentiles ensures that the PC is close to an upper envelope of the spectrum in the PC ranges. 

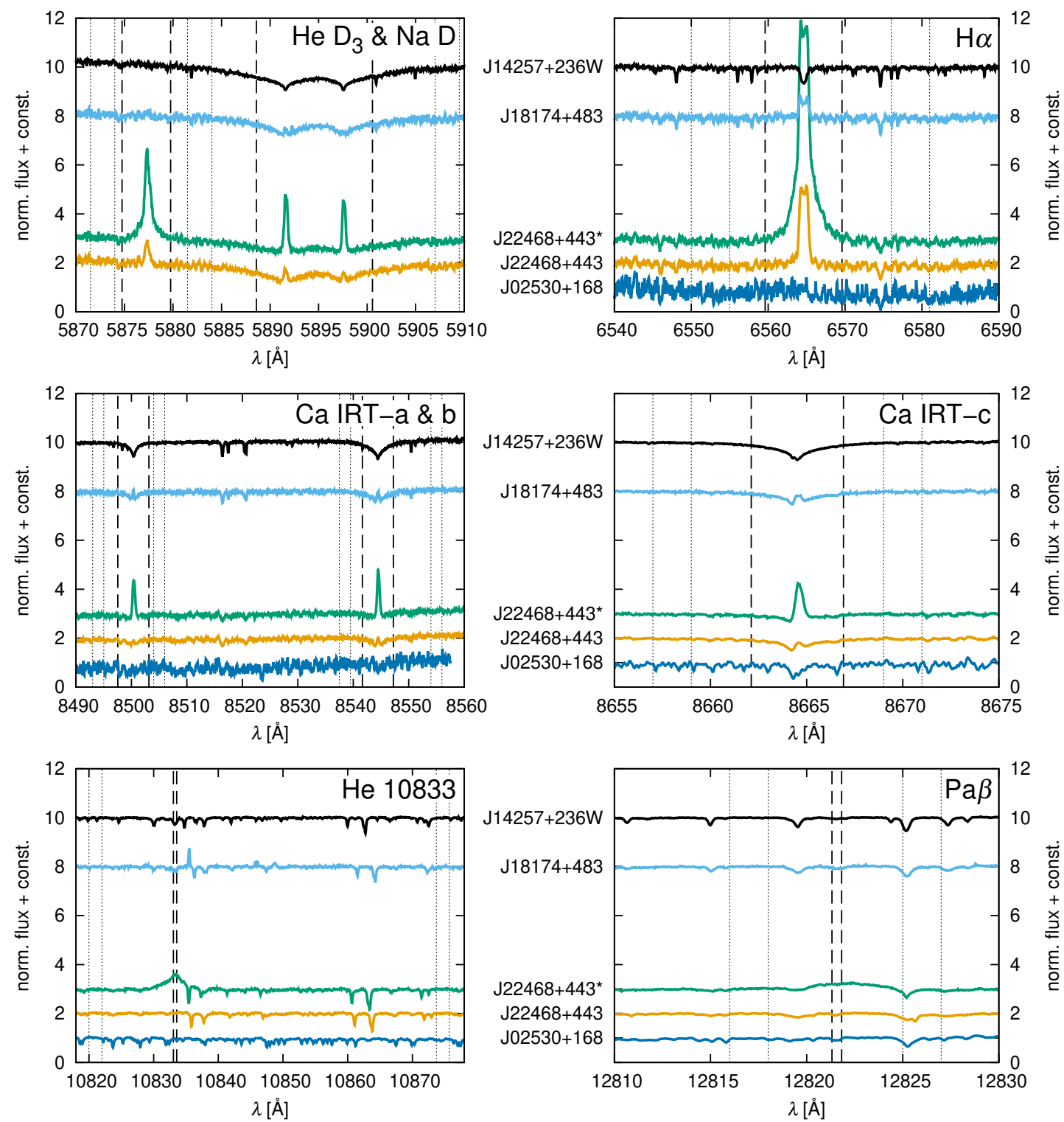

Figure 2.2.: Selected spectra in regions around the eight considered chromospheric lines: J14257+236W (M0.0, black), J18174+483 (M2.0, light blue), J22468+443 (M3.5) in flaring (green) and quiescent (orange) state, J02530+168 (M7.0, dark blue). J02530+168 is not shown in the region around $\mathrm{He}_{3}$ and $\mathrm{Na}$ D because of low SNR. Dashed vertical lines encompass the line windows, dotted vertical lines the pseudo-continuum ranges as defined in Table 2.1. 
Table 2.1.: Line windows and pseudo-continuum ranges for $\mathrm{pEW}^{\prime}$ measurements of selected lines with chromospheric components. All wavelengths are given in vacuum.

\begin{tabular}{lccccc}
\hline Line & $\lambda_{0}[\AA]$ & $\Delta \lambda[\AA]$ & $\lambda_{1}[\AA]$ & $\lambda_{\mathrm{r}}[\AA]$ & $\Delta \lambda_{\mathrm{PC}}[\AA]$ \\
\hline $\mathrm{He} \mathrm{D}_{3}$ & 5877.25 & 2.50 & 5872.75 & 5882.75 & 1.25 \\
$\mathrm{Na} \mathrm{D}$ & 5894.57 & 5.98 & 5882.75 & 5908.25 & 1.25 \\
$\mathrm{H} \alpha$ & 6564.60 & 5.00 & 6552.50 & 6578.50 & 2.50 \\
$\mathrm{Ca}$ IRT-a & 8500.35 & 2.80 & 8494.00 & 8505.00 & 1.00 \\
$\mathrm{Ca}$ IRT-b & 8544.44 & 2.80 & 8538.50 & 8555.00 & 1.00 \\
$\mathrm{Ca}$ IRT-c & 8664.52 & 2.40 & 8658.00 & 8670.00 & 1.00 \\
\hline $\mathrm{He} \mathrm{10833}$ & 10833.31 & 0.25 & 10821.0 & 10874.7 & 1.00 \\
$\mathrm{~Pa} \beta$ & 12821.57 & 0.25 & 12817.0 & 12826.0 & 1.00 \\
\hline
\end{tabular}

The $\mathrm{pEW}^{\prime}$ was then calculated as

$$
\mathrm{pEW}^{\prime}=-\int_{\lambda_{0}-\Delta \lambda}^{\lambda_{0}+\Delta \lambda}\left(\frac{S(\lambda)}{\mathrm{PC}(\lambda)}-\frac{T(\lambda)}{\mathrm{PC}(\lambda)}\right) \mathrm{d} \lambda
$$

using the trapezoidal rule to approximate the integral, where $S(\lambda)$ is the normalised investigated spectrum and $T(\lambda)$ is the normalised reference star spectrum. The line windows $\lambda_{0} \pm \Delta \lambda$ are listed in Table 2.1. The $\mathrm{pEW}^{\prime}$ is defined to be negative in the case of excess emission to be consistent with the traditional definition of the EW. Using the inverse root mean square $(r m s)$ of the PC ranges in the residual spectrum $R(\lambda)=S(\lambda)-T(\lambda)$ as an approximation for the SNR, the uncertainty of the $\mathrm{pEW}^{\prime}$ was estimated following Vollmann \& Eversberg (2006) as

$$
\epsilon_{\mathrm{pEW}}=\sqrt{1+\frac{\mathrm{PC}\left(\lambda_{0}\right)}{\langle R\rangle+1}} \cdot r m s \cdot\left(2 \Delta \lambda-\mathrm{pEW}^{\prime}\right),
$$

where $\langle R\rangle$ is the mean of $R(\lambda)$ in the line window $\lambda_{0} \pm \Delta \lambda$.

In contrast to a $\mathrm{pEW}$ measurement without spectral subtraction, the $\mathrm{pEW}$ measurement is less affected by photospheric absorption components of spectral features such as $\mathrm{Na} D$ or Ca IRT as long as these components are sufficiently similar in the considered spectrum and the reference star spectrum. Similarly, the spectral subtraction mitigates the impact of any absorption band lines close to the considered spectral line from the line window. This allows the choice of sufficiently broad line windows to measure most of the chromospheric emission even during flaring events, which can lead to very broad emission lines as seen in Fig. 1.12. Without the spectral subtraction, a broad line window can introduce a spectral-type dependent bias, as the strength of included absorption band lines can depend on the temperature. Alternatively, a variable line window chosen based on the width of the line in each spectrum separately could mitigate this effect, but this would lose the advantage that the $\mathrm{pEW}$ method requires little information on the line shape. 
Both the $\mathrm{He} 10833$ and the $\mathrm{Pa} \beta$ line are weak in comparison to nearby telluric lines that appear in the line windows for absolute observed RVs between $14 \mathrm{~km} \mathrm{~s}^{-1}$ and $125 \mathrm{~km} \mathrm{~s}^{-1}$ for He 10833, and between $10 \mathrm{~km} \mathrm{~s}^{-1}$ and $43 \mathrm{~km} \mathrm{~s}^{-1}$ for $\mathrm{Pa} \beta$. I exclude any spectra with telluric contamination based on the absolute observed RV from further analysis for these lines.

For $\mathrm{H} \alpha$ as the most commonly used activity indicator for M dwarfs, I defined an activity threshold of $\mathrm{pEW}_{\mathrm{H} \alpha}^{\prime}=-0.3 \AA$. Stars with a stronger excess emission are $\mathrm{H} \alpha$ active, whereas stars with a weaker excess emission or with excess absorption are $\mathrm{H} \alpha$ inactive. Previous studies defined different activity thresholds for the $\mathrm{pEW}$ of $\mathrm{H} \alpha$ without spectral subtraction, e.g. $-1 \AA$ (Newton et al. 2017) or $-0.5 \AA$ (Jeffers et al. 2018). However, I will show that a lower threshold for the excess emission is justified in Sect. 3.3.

\subsubsection{Grouping of stars and selection of reference stars}

By definition, the $\mathrm{pEW}^{\prime}$ of all lines is $0 \AA$ for reference stars. To obtain meaningful results for as many stars as possible, the stars thus have to be grouped into as few groups as possible. As the strength of the absorption lines that the spectral subtraction aims to remove depends mainly on the temperature, I grouped the stars by their spectral subtypes given in Carmencita as listed in Table A.1. However, all stars with spectral subtype M6.0 or later were merged into one group because the sample of very late-type stars is sparse and inactive stars that could be used as reference stars are elusive. This introduces a bias in the results for very late-type stars, as the surrounding lines in the reference star spectrum may be stronger or weaker than in the investigated spectrum. Alternatively, the stars could be grouped by effective temperature. However, while the effective temperature was determined by Schweitzer et al. (2019) for most of the 337 sample stars, this would not solve the problem with the coolest, very late-type stars, and a reasonable binning would lead to groups that are similar to the spectral subtype groups.

Ideally, the reference star should be the most inactive star in each group, so the spectral subtraction does not remove any sign of activity. However, there are multiple ways to define the most inactive stars. Given the correlation between chromospheric activity and stellar rotation described in Sect. 1.4.2, I generally selected for each subtype the star with the longest rotation period as given in Carmencita and tabulated in Table A.1. For spectral subtype M1.5, J16254+543 was chosen as the reference star instead of J03181+382 $\left(P_{\text {rot }}=[77.2 \pm 3.0] \mathrm{d}\right)$ based on a longer reported rotation period of $(100 \pm 5) \mathrm{d}$ that was revised later. The rotation periods of J23381-162 $\left(P_{\text {rot }}=61.66 \mathrm{~d}\right)$ and J09360-216 $\left(P_{\text {rot }}=[74.3 \pm 1.7] \mathrm{d}\right)$ were not included in Carmencita when the reference stars were selected, so J06103+821 and J17198+417 were chosen instead. For M5.5 and M6.0, the only stars with $\mathrm{H} \alpha$ not in emission were selected. The selected reference stars and their rotation periods $P_{\text {rot }}$ are listed in Table 2.2.

For each sample star, the reference star of the same spectral subtype should show the most similar spectrum in the temperature-dependent PC ranges. Therefore, I calculated 
Table 2.2.: Reference stars with the longest rotation periods and with the smallest MNI variations for each spectral subtype. Both sets of reference stars contain the same star for spectral subtypes M5.5 and M6.0. The same reference star is used for all spectral subtypes later than M6.0.

\begin{tabular}{llcllc}
\hline & \multicolumn{2}{c}{ longest $P_{\text {rot }}$} & & \multicolumn{2}{c}{ smallest MNI variation } \\
\cline { 2 - 3 } \cline { 5 - 6 } Spectral type & Reference star & $P_{\text {rot }}[\mathrm{d}]$ & & Reference star & $P_{\text {rot }}[\mathrm{d}]$ \\
\hline M0.0 & $\mathrm{J} 14257+236 \mathrm{~W}$ & $111 \pm 12$ & & $\mathrm{~J} 09144+526$ & $\ldots$ \\
M0.5 & $\mathrm{J} 18580+059$ & $35.2 \pm 0.3$ & & $\mathrm{~J} 22503-070$ & $\ldots$ \\
M1.0 & $\mathrm{J} 18051-030$ & $127.8 \pm 3.2$ & & $\mathrm{~J} 13209+342$ & $\ldots$ \\
M1.5 & $\mathrm{J} 16254+543$ & $76.79 \pm 0.13$ & & $\mathrm{~J} 17052-050$ & $50.2 \pm 1.3$ \\
M2.0 & $\mathrm{J} 06103+821$ & $44.6 \pm 1.0$ & & $\mathrm{~J} 22115+184$ & $36.3 \pm 0.2$ \\
M2.5 & $\mathrm{J} 17198+417$ & $71.5 \pm 2.6$ & & $\mathrm{~J} 19169+051 \mathrm{~N}$ & $46.0 \pm 0.2$ \\
M3.0 & $\mathrm{J} 15194-077$ & $132.5 \pm 6.3$ & & $\mathrm{~J} 18419+318$ & $\ldots$ \\
M3.5 & $\mathrm{J} 17578+046$ & $148.6 \pm 0.1$ & & $\mathrm{~J} 14310-122$ & $\ldots$ \\
M4.0 & $\mathrm{J} 11477+008$ & $163 \pm 3$ & & $\mathrm{~J} 21466-001$ & $\ldots$ \\
M4.5 & $\mathrm{J} 19216+208$ & $133 \pm 9$ & & $\mathrm{~J} 19098+176$ & $80.1 \pm 3.2$ \\
M5.0 & $\mathrm{J} 03133+047$ & 126.2 & & $\mathrm{~J} 18027+375$ & 123.8 \\
M5.5 & $\mathrm{J} 00067-075$ & $\ldots$ & & $\mathrm{J} 00067-075$ & $\ldots$ \\
M6.0 & $\mathrm{J} 07403-174$ & $\ldots$ & & $\mathrm{J} 07403-174$ & $\ldots$ \\
\hline
\end{tabular}

the rms of the PC ranges in the residual spectra after subtracting each of the reference star spectra from the co-added template spectra for all sample stars as a sanity check. In Fig. 2.3, I show the resulting rms of the PC ranges around the $\mathrm{H} \alpha$ line for all M3.0 stars as a function of the spectral subtype of the reference star. The rms is minimised by using the M3.0 reference star for 37 of 43 M3.0 stars in the sample, whereas it is minimised by using the M3.5 reference star for 5 stars and by using the M2.5 reference star for only one star. As shown by the histograms for all considered lines in Fig. 2.4, it generally holds true for all lines and all spectral subtype that the $r m s$ is minimised by using a reference star of a similar spectral type. The deviations are larger for $\mathrm{He}_{3}, \mathrm{Na} D, \mathrm{He} 10833$ and $\mathrm{Pa} \beta$ than for $\mathrm{H} \alpha$ and the Ca IRT lines. This can be explained by the lower SNR at the short wavelengths of $\mathrm{He}_{3}$ and $\mathrm{Na} \mathrm{D}$, particularly at later spectral subtypes, and the comparatively flat $\mathrm{PC}$ regions for $\mathrm{He} 10833$ and $\mathrm{Pa} \beta$. For spectral types later than M6.0, the rms is always minimised by an earlier-type reference star, because there are no later-type reference stars. Similarly, for M0.0 stars, the $r m s$ is always minimised by the reference star of the same or a later spectral subtype.

As the rotation period is known for only 169 of the 337 sample stars, this selection of reference stars may be biased. It is possible that the remaining stars are even less active and show less brightness variation, so the rotation period could not be measured from photometric data. To investigate the impact of the reference stars, I selected a second set of reference stars based on the molecular-normalised index (MNI) of the $\mathrm{H} \alpha$ line defined and calculated by Erik Johnson (Institut für Astrophysik Göttingen). The MNI is calculated by dividing the mean flux in the line window by the mean flux in two PC regions on either side of the line window and dividing again by the mean flux in two 


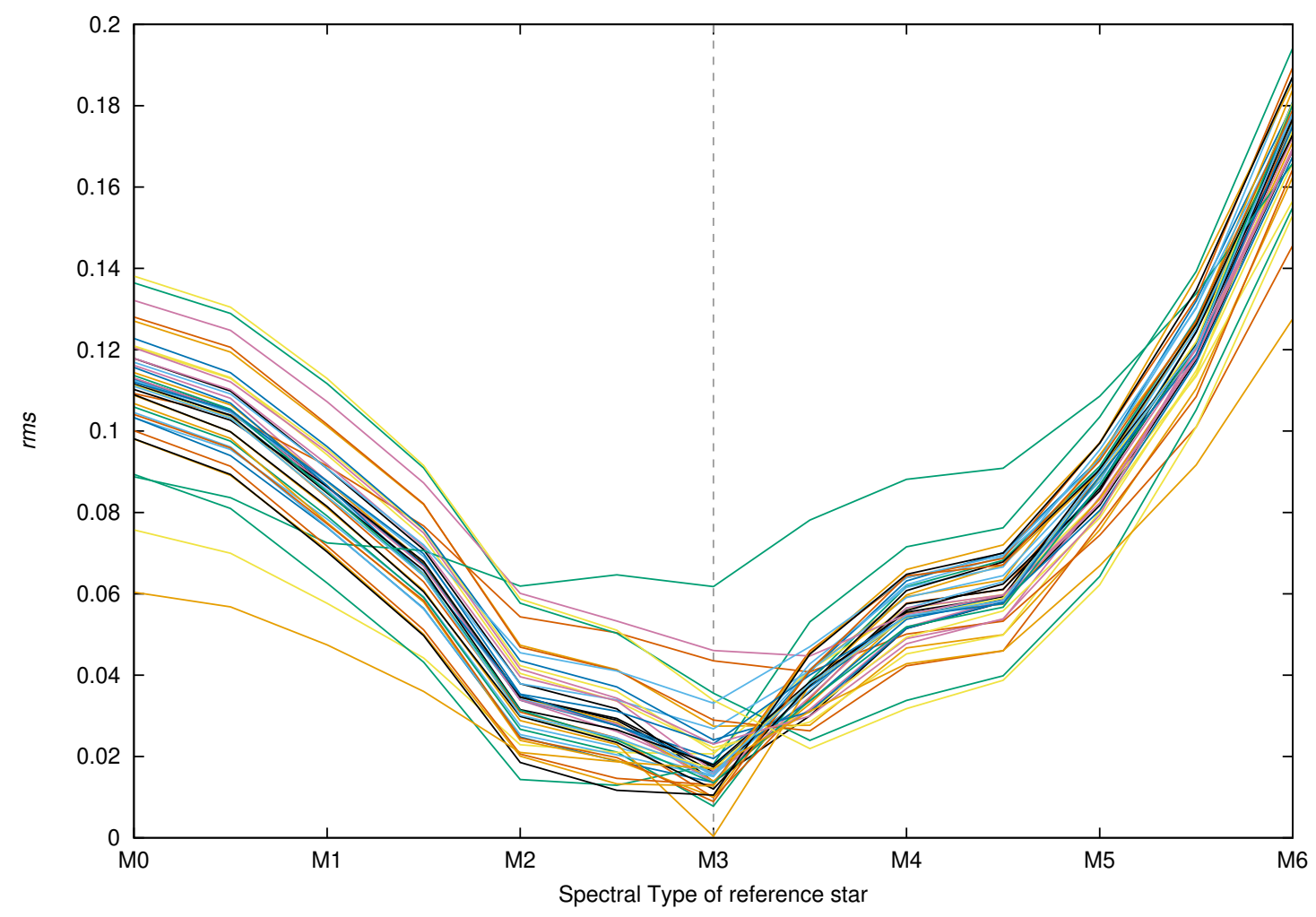

Figure 2.3.: rms of $\mathrm{PC}$ ranges in residual spectra around the $\mathrm{H} \alpha$ line after subtraction of different reference star spectra for all M3.0 stars in the sample as a function of the spectral subtype of the reference star. To improve the visibility of the minima, the measurements for each star are connected with a curve. 


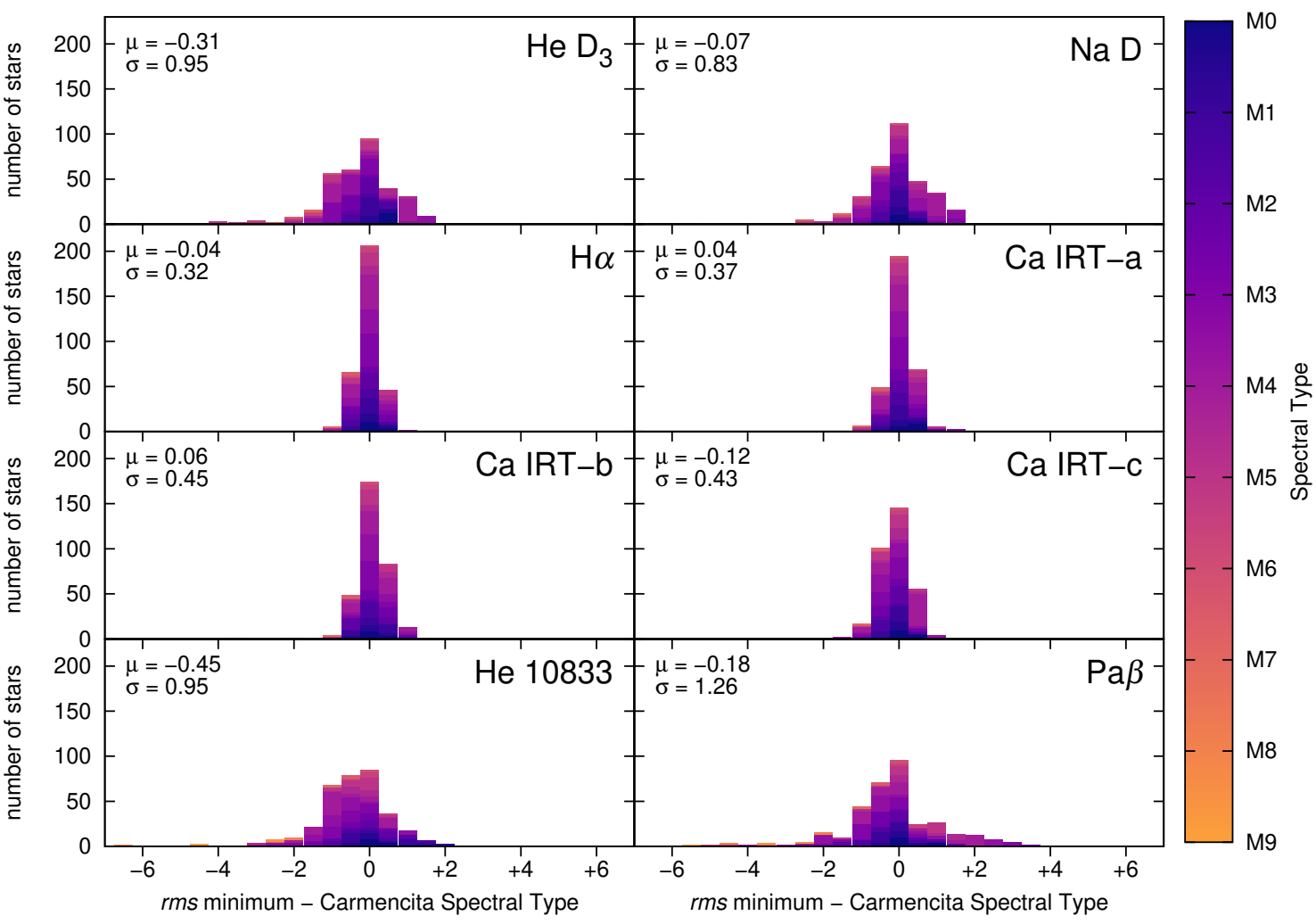

Figure 2.4.: Histogram of deviations between spectral subtype of the reference star that minimises the rms of the PC ranges in the residual spectra and spectral subtype according to Carmencita.

further PC regions, and is less variable for less active stars. Therefore, the stars with the smallest MNI variation are selected as a second set of reference stars and also listed in Table 2.2. Their rotation periods are listed for comparison, but are unknown for eight of the thirteen stars. For three of the five stars with a known rotation period, the period is less than two thirds of the rotation period of the reference star with the longest period of that subtype.

\subsubsection{Normalised $\mathrm{H} \alpha$ luminosity}

If two $\mathrm{pEW}^{\prime}$ values of the same line in different stars were derived using different reference stars, a direct comparison is difficult because the PC changes with the effective temperature similar to Eq. 1.1. This is similar to the changes in the black-body radiation that were described in Sect. 1.1. To remove this temperature dependence, I converted the $\mathrm{pEW}_{\mathrm{H} \alpha}^{\prime}$ values to the normalised $\mathrm{H} \alpha$ luminosity $L_{\mathrm{H} \alpha} / L_{\mathrm{bol}}$ using the ratio $\chi$ between the PC flux around $\mathrm{H} \alpha$ and the bolometric flux as described by Walkowicz et al. (2004):

$$
\frac{L_{\mathrm{H} \alpha}}{L_{\mathrm{bol}}}=-\frac{\mathrm{pEW}_{\mathrm{H} \alpha}^{\prime}}{1 \AA} \cdot \chi\left(T_{\mathrm{eff}}\right),
$$


where I used a quintic function derived from PHOENIX model spectra by Reiners \& Basri (2008) for the flux ratio $\chi\left(T_{\text {eff }}\right)$, and $T_{\text {eff }}$ based on the spectral subtype of the reference star as given by Pecaut \& Mamajek (2013) and tabulated in Table 1.1. Because the normalised $\mathrm{H} \alpha$ luminosity is usually given on a logarithmic scale, I only calculated it for $\mathrm{H} \alpha$ active stars, as $\log \left(L_{\mathrm{H} \alpha} / L_{\mathrm{bol}}\right)$ goes to minus infinity as $\mathrm{pEW}_{\mathrm{H} \alpha}^{\prime}$ approaches $0 \AA$.

\subsection{Indices of photospheric bands}

As discussed in Sect. 1.2.3, molecular absorption bands formed in the photosphere are dominant features in $\mathrm{M}$ dwarf spectra. An absorption band can extend over several Echelle orders, complicating the definition of a pseudo-continuum for the $\mathrm{pEW}^{\prime}$ method described in the previous section. Moreover, a wide absorption band likely blends with other spectral features, thus also complicating the choice of an integration window for measuring the strength of the absorption band only. Therefore, I quantified the absorption band strength by an index that is calculated as the flux ratio of two small spectral ranges on either side of the band head:

$$
\text { index }=\frac{\langle S\rangle_{\text {num. }}}{\langle S\rangle_{\text {den. }}},
$$

where $\langle S\rangle$ is the mean flux in the numerator or denominator range. To mitigate the impact of ill data points from cosmic rays or detector artefacts, the top and bottom 10 percent of the data points in each range are not considered for the mean.

The uncertainty of the index is given by

$$
\epsilon_{\text {index }}=\operatorname{index} \sqrt{\left(\frac{\epsilon_{\text {num. }}}{\langle S\rangle_{\text {num. }}}\right)^{2}+\left(\frac{\epsilon_{\text {den. }}}{\langle S\rangle_{\text {den. }}}\right)^{2}}
$$

from propagating the errors of each mean flux. According to Zechmeister et al. (2018), these can be estimated from the data error $\epsilon_{i}$ of each spectral bin as

$$
\epsilon_{\langle S\rangle}=\frac{1}{N} \sqrt{\sum \epsilon_{i}^{2}}
$$

Similar indices have been defined and used for deriving spectral types (e.g. Reid et al. 1995; Martín et al. 1999) and metallicities (e.g. Woolf \& Wallerstein 2006; Lépine et al. 2007) before, but with broader or farther apart numerator and denominator ranges tailored to low-resolution spectra. To measure the strength of each band head as accurately as possible in high-resolution spectra, I defined new indices using narrower spectral ranges as given in Table 2.3. I considered the strongest band head of the $\mathrm{TiO} \gamma, \delta$, and $\epsilon$ systems according to Valenti et al. (1998); their strength is measured by the TiO 7050, TiO 8860, and $\mathrm{TiO} 8430$ indices, respectively. While the strongest band heads of the $\mathrm{TiO} \alpha, \beta$, and $\gamma^{\prime}$ systems are also covered by the VIS channel, they are located at shorter wavelengths than the $\mathrm{H} \alpha$ line and hence in a spectral range with a poor SNR for the later-type stars. 
Table 2.3.: Numerator and denominator ranges for photospheric absorption band indices. All wavelengths are given in vacuum.

\begin{tabular}{lcc}
\hline Index & Numerator $[\AA]$ & Denominator $[\AA]$ \\
\hline CaH 2 $^{*}$ & $6817.0-6819.0$ & $6813.0-6815.0$ \\
CaH 3 $^{*}$ & $6974.0-6977.0$ & $7047.0-7050.0$ \\
TiO 7050 & $7056.0-7060.0$ & $7046.0-7050.0$ \\
VO 7436 & $7435.9-7436.9$ & $7434.3-7435.3$ \\
VO 7942 & $7941.7-7943.7$ & $7936.0-7938.0$ \\
TiO 8430 & $8436.0-8440.0$ & $8430.5-8434.5$ \\
TiO 8860 & $8862.5-8864.5$ & $8859.0-8861.0$ \\
\hline FeH WFB & $9898.0-9907.0$ & $9887.0-9896.0$ \\
\hline
\end{tabular}

Therefore, I consider neither these systems nor the $\mathrm{TiO} \phi$ system, which is covered by the NIR channel but located in a range with strong telluric contamination. The VO band heads listed by Tinney \& Reid (1998) are more diffuse and many of them are located within TiO bands. I defined the VO 7436 and VO 7942 indices to measure the strengths of two $\mathrm{VO}$ band heads not blended with $\mathrm{TiO}$ bands. In addition to the $\mathrm{TiO}$ and $\mathrm{VO}$ indices, I defined high-resolution counterparts of the $\mathrm{CaH} 2$ and $\mathrm{CaH} 3$ indices from Reid et al. (1995) and measured the strength of the FeH Wing-Ford band (first detected by Wing \& Ford 1969) with the FeH WFB index, which is the only index derived from NIR spectra. All considered bands are visualised for selected stars in Fig. 2.5. 

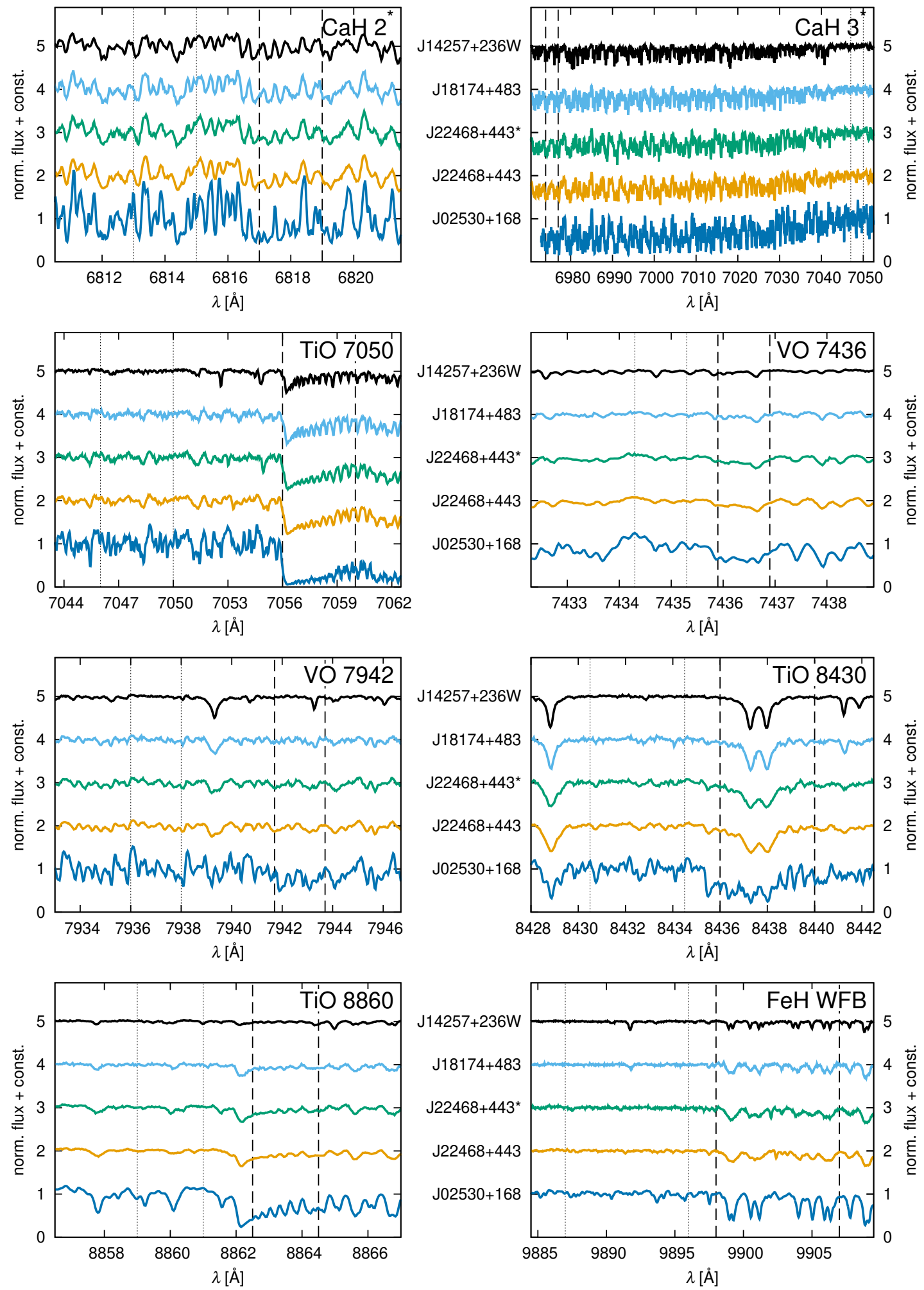

Figure 2.5.: Same spectra as in Fig. 2.2 in regions around the eight considered photospheric bands: Dashed vertical lines encompass the numerator ranges, dotted vertical lines the denominator ranges as defined in Table 2.3. 


\section{Chromospheric indicators}

This chapter aims to quantify the chromospheric activity in the CARMENES sample of the $\mathrm{M}$ dwarfs. As described in Sect. 1.2.4, the chromosphere is the atmospheric layer above the photosphere. Several spectral lines that are sensitive to the hotter temperatures in comparison to the photosphere can be used as indicators for chromospheric activity as explained in Sect. 1.4.1.

I present and analyse the chromospheric activity indicators derived with the spectral subtraction method described in Sect. 2.2. As a starting point, I show the $\mathrm{pEW}^{\prime}$ values derived from each star's co-added template spectrum in Sect. 3.1. I then explore the correlations between the $\mathrm{pEW}^{\prime}$ of different lines in Sect. 3.2, before quantifying the variations among individual observations of each star in Sect. 3.3. These three sections mostly follow the analysis presented in Schöfer et al. (2019), but include also observations after July 2018 and all spectra have been reprocessed using the latest co-added template spectra of the reference stars. In Sect. 3.4, I demonstrate how the choice of the reference stars affects the results. Finally, I provide a comparison with previous results and a discussion of the findings in Sect. 3.5.

\subsection{Sample overview}

For all eight considered spectral lines, Fig. 3.1 shows the $\mathrm{pEW}^{\prime}$ values derived from the co-added template spectra grouped by spectral subtype using the reference stars with the longest rotation periods per spectral subtype. The $\mathrm{pEW}_{\mathrm{H} \alpha}^{\prime}$ values are also tabulated in Table A.1. As defined in Sect. 2.2, negative $\mathrm{pEW}^{\prime}$ values mean excess emission and positive $\mathrm{pEW}^{\prime}$ values mean excess absorption with respect to the reference star for the same spectral subtype, which by definition has $\mathrm{pEW}^{\prime}=0 \AA$ for all lines. Stars with $\mathrm{H} \alpha$ excess emission stronger than the activity threshold of $-0.3 \AA$ are marked as $\mathrm{H} \alpha$ active.

All $\mathrm{H} \alpha$ inactive stars show only minor deviations from $\mathrm{pEW}_{\mathrm{He}_{3}}^{\prime}=0 \AA$. In contrast, most $\mathrm{H} \alpha$ active stars show stronger excess emission in $\mathrm{He}_{3} \mathrm{D}_{3}$ than any $\mathrm{H} \alpha$ inactive star. The maximum observed excess emission per spectral subtype tends to increase with the spectral subtype up to a maximum at M5.5. Na D behaves in a similar way to $\mathrm{He} \mathrm{D}_{3}$, but with a less clear separation between $\mathrm{H} \alpha$ active and $\mathrm{H} \alpha$ inactive stars at early and mid subtypes and excess absorption in two very late $\mathrm{H} \alpha$ active stars. In addition, there is a larger spread for $\mathrm{H} \alpha$ inactive stars; in particular, all other $\mathrm{H} \alpha$ inactive M1.5 stars show significant $\mathrm{Na} \mathrm{D}$ excess emission with respect to the reference star for this subtype. 

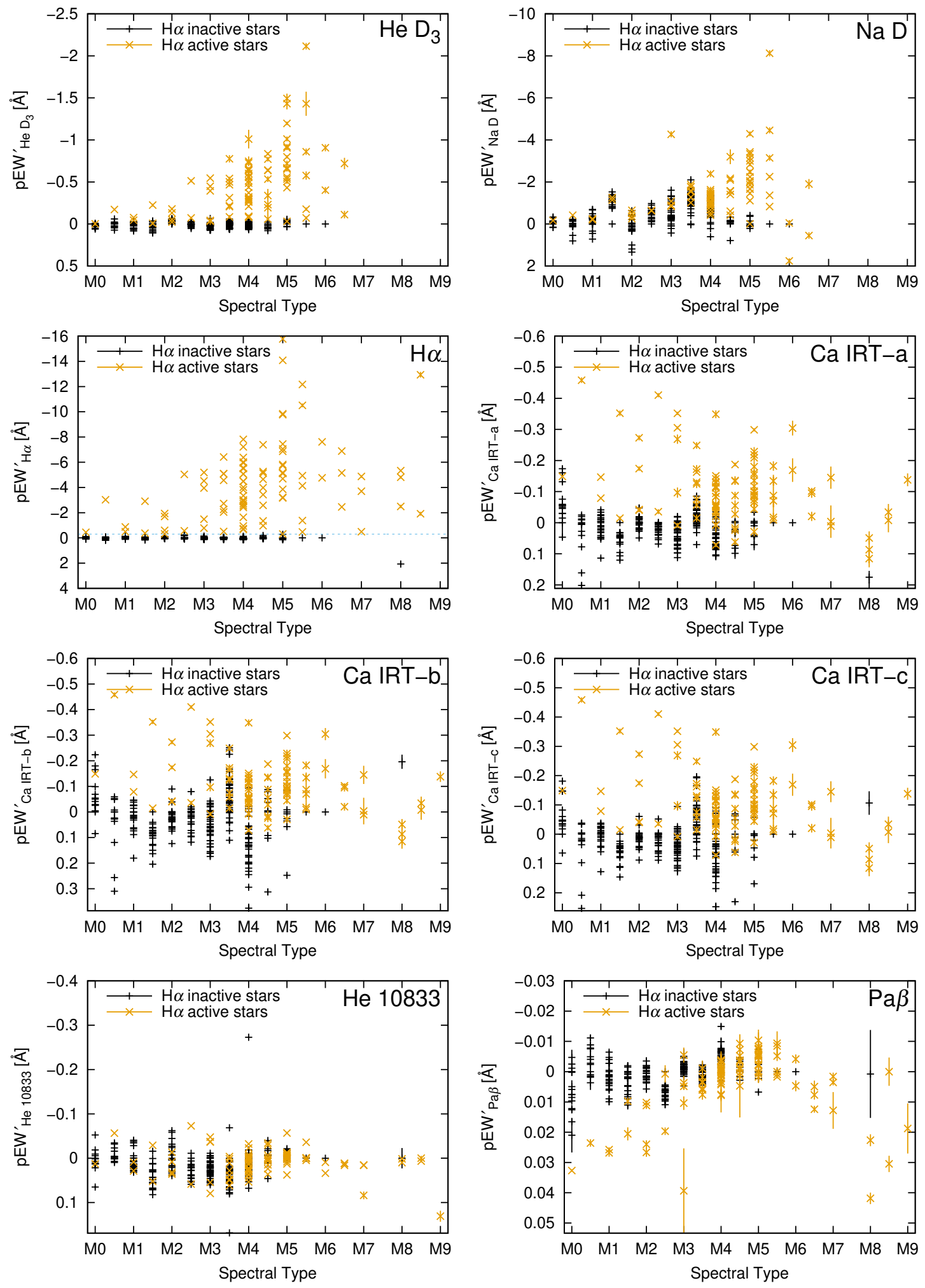

Figure 3.1.: $\mathrm{pEW}^{\prime}$ of spectral lines with a chromospheric component as a function of spectral type for $\mathrm{H} \alpha$ inactive stars (black pluses) and $\mathrm{H} \alpha$ active stars (orange crosses). The dashed blue line in the $\mathrm{H} \alpha$ panel marks the activity threshold at $\mathrm{pEW}_{\mathrm{H} \alpha}^{\prime}=-0.3 \AA$. 

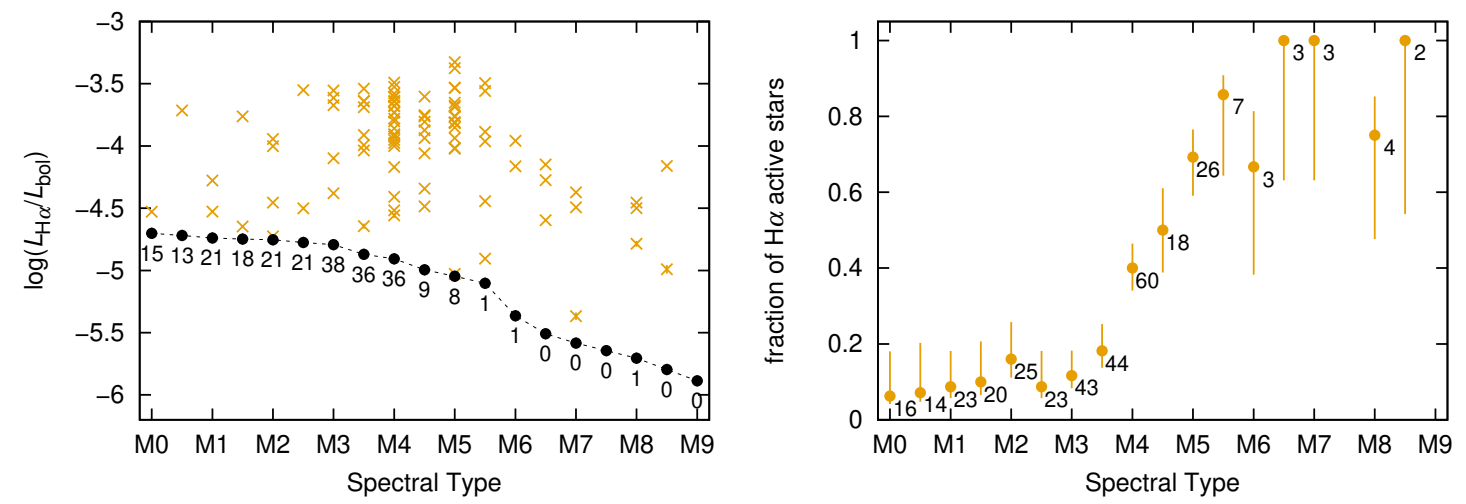

Figure 3.2.: Left panel: normalised $\mathrm{H} \alpha$ luminosity of $\mathrm{H} \alpha$ active stars as a function of spectral type. The black circles correspond to the activity threshold and the numbers are the number of $\mathrm{H} \alpha$ inactive stars for each spectral subtype. Right panel: fraction of $\mathrm{H} \alpha$ active stars as a function of spectral type with error bars from binomial statistics. The numbers indicate the total number of sample stars for each spectral subtype.

Unlike $\mathrm{He}_{3}$ and $\mathrm{Na} \mathrm{D}, \mathrm{H} \alpha$ could be measured also for nine very late-type stars between M7.0 and M8.5. Among these stars, only J19255+096 is classified as H $\alpha$ inactive with the strongest $\mathrm{H} \alpha$ excess absorption of all sample stars. The overall distribution of the measured $\mathrm{pEW}_{\mathrm{H} \alpha}^{\prime}$ values among spectral subtypes is similar to $\mathrm{He} \mathrm{D}_{3}$, but with the global maximum observed excess emission at M5.0 instead of M5.5. This difference can be explained with the different flux ratios of the pseudo-continua. The normalised $\mathrm{H} \alpha$ luminosity $L_{\mathrm{H} \alpha} / L_{\mathrm{bol}}$ was calculated as described in Sect. 2.2.2 and is shown as a function of spectral type for all $\mathrm{H} \alpha$ active stars in the left panel of Fig. 3.2 and tabulated in Table A.1. In general, later-type stars have a lower normalised $\mathrm{H} \alpha$ luminosity than earlier-type stars with a comparable $\mathrm{pEW}_{\mathrm{H} \alpha}^{\prime}$. The M5.0 star with the most negative $\mathrm{pEW}_{\mathrm{H} \alpha}^{\prime}, \mathrm{J} 05084-210$, also has the highest normalised $\mathrm{H} \alpha$ luminosity of all sample stars at $\log \left(L_{\mathrm{H} \alpha} / L_{\mathrm{bol}}\right)=-3.33$. The right panel of Fig. 3.2 shows the fraction of $\mathrm{H} \alpha$ active stars for each spectral subtype. While below $20 \%$ of the stars are $\mathrm{H} \alpha$ inactive for each spectral subtype up to M3.5, the fraction increases rapidly for subtypes M4.0 to M5.5 up to more than $80 \%$. Almost all very late-type stars with subtype M6.0 or later are classified as $\mathrm{H} \alpha$ active, but the sample is too sparse to rule out that the fraction decreases again. Overall, 95 of 334 sample stars with a measured $\mathrm{pEW}_{\mathrm{H} \alpha}^{\prime}$ are classified as $\mathrm{H} \alpha$ active.

The other spectral lines shown in Fig. 3.1 are located at longer wavelengths than $\mathrm{H} \alpha$, so the $\mathrm{pEW}^{\prime}$ for the only M9.0 and the latest-type star in the sample, J08536-034, could be measured. Although $\mathrm{pEW}_{\mathrm{H} \alpha}^{\prime}$ could not be measured for this star, as the order containing the $\mathrm{H} \alpha$ line is not included in the co-added template spectrum, it is marked as an $\mathrm{H} \alpha$ active star based on visual inspection of the $\mathrm{H} \alpha$ line in individual spectra. All three Ca IRT lines show a similar distribution of the measured $\mathrm{pEW}^{\prime}$ as a function of spectral subtype. Most $\mathrm{H} \alpha$ active stars show excess emission, with earlier-type stars showing a stronger maximum excess emission as later-type stars. Again, this difference to the previously described indicators can be explained with the different pseudo-continuum flux ratios. 
Table 3.1.: Correlation table of $\mathrm{pEW}^{\prime}$ values of spectral lines with a chromospheric component using Spearman's rank coefficient $r_{S}$. Strong correlations with $r_{S}>0.60$ are highlighted in boldface.

\begin{tabular}{lrrrrrrr}
\hline & $\mathrm{H} \alpha$ & $\mathrm{He} \mathrm{D}_{3}$ & $\mathrm{Na} \mathrm{D}$ & Ca IRT-a & Ca IRT-b & Ca IRT-c & He 10833 \\
\hline $\mathrm{He} \mathrm{D}_{3}$ & $\mathbf{0 . 6 8}$ & $\ldots$ & & & & & \\
$\mathrm{Na} \mathrm{D}$ & 0.23 & 0.26 & $\ldots$ & & & & \\
Ca IRT-a & $\mathbf{0 . 7 3}$ & $\mathbf{0 . 6 6}$ & 0.22 & $\ldots$ & & & \\
Ca IRT-b & $\mathbf{0 . 6 3}$ & 0.41 & 0.15 & $\mathbf{0 . 7 7}$ & $\ldots$ & & \\
Ca IRT-c & 0.41 & 0.22 & 0.03 & $\mathbf{0 . 6 1}$ & $\mathbf{0 . 9 1}$ & $\ldots$ & \\
$\mathrm{He} \mathrm{10833}$ & 0.23 & 0.15 & -0.33 & 0.22 & 0.12 & 0.06 & $\ldots$ \\
$\mathrm{Pa} \beta$ & -0.18 & -0.08 & 0.13 & -0.13 & -0.26 & -0.28 & 0.34 \\
\hline
\end{tabular}

Similar to $\mathrm{Na} \mathrm{D}$, the $\mathrm{H} \alpha$ inactive stars show a larger spread in the Ca IRT lines than in $\mathrm{He} \mathrm{D}_{3}$ or $\mathrm{H} \alpha$. While most $\mathrm{H} \alpha$ inactive stars show excess emission with respect to the reference star for spectral subtypes M0.0 and M3.5, excess absorption is more common in $\mathrm{H} \alpha$ inactive stars for M1.0 and M4.0.

For the two lines in the range of the NIR channel, He 10833 and $\mathrm{Pa} \beta$, the spectral-type dependence of the measured $\mathrm{pEW}^{\prime}$ values is significantly different than for the other lines. In $\mathrm{He} 10833$, the $\mathrm{H} \alpha$ active stars are not separated from the $\mathrm{H} \alpha$ inactive stars. In $\mathrm{Pa} \beta$, most $\mathrm{H} \alpha$ active stars show excess absorption at early to mid types and at very late types, whereas the $\mathrm{pEW}_{\mathrm{Pa} \beta}^{\prime}$ values of $\mathrm{H} \alpha$ active stars at mid to late types are similar to the values of $\mathrm{H} \alpha$ inactive stars.

\subsection{Correlations}

As seen in the previous section, there are similarities in the distributions of the measured $\mathrm{pEW}^{\prime}$ values of different spectral lines. This suggests that these indicators are not independent of each other. To investigate the correlations, I show scatter plots of $\mathrm{pEW}_{\mathrm{H} \alpha}^{\prime}$ paired with all other indicators in Fig. 3.3, and of $\mathrm{pEW}_{\mathrm{He}_{3}}^{\prime}$ versus $\mathrm{pEW}_{\mathrm{He} 10833}^{\prime}$ and of all combinations of the Ca IRT lines in Fig. 3.4. As a more quantitative approach, I also calculated the correlation coefficients between all pairs of indicators. Because the distributions of most indicators are heavy-tailed with the $\mathrm{H} \alpha$ inactive stars clustered around $0 \AA$ and a long tail of $\mathrm{H} \alpha$ active stars, I use Spearman's rank coefficient $r_{S}$ rather than Pearson's correlation coefficient. The resulting correlation table is given in Table 3.1. In addition, a correlation table for only the $\mathrm{H} \alpha$ active stars is given in Table 3.2.

The first scatter plot in the left column of Fig. 3.3 shows a strong correlation between $\mathrm{H} \alpha$ and $\mathrm{He} \mathrm{D}_{3}$. The correlation coefficient is $r_{S}=0.68$ and increases to $r_{S}=0.95$ if the $\mathrm{H} \alpha$ inactive stars are excluded. This is the strongest correlation measured for any pair of chromospheric indicators. No different slopes for different spectral subtypes are 

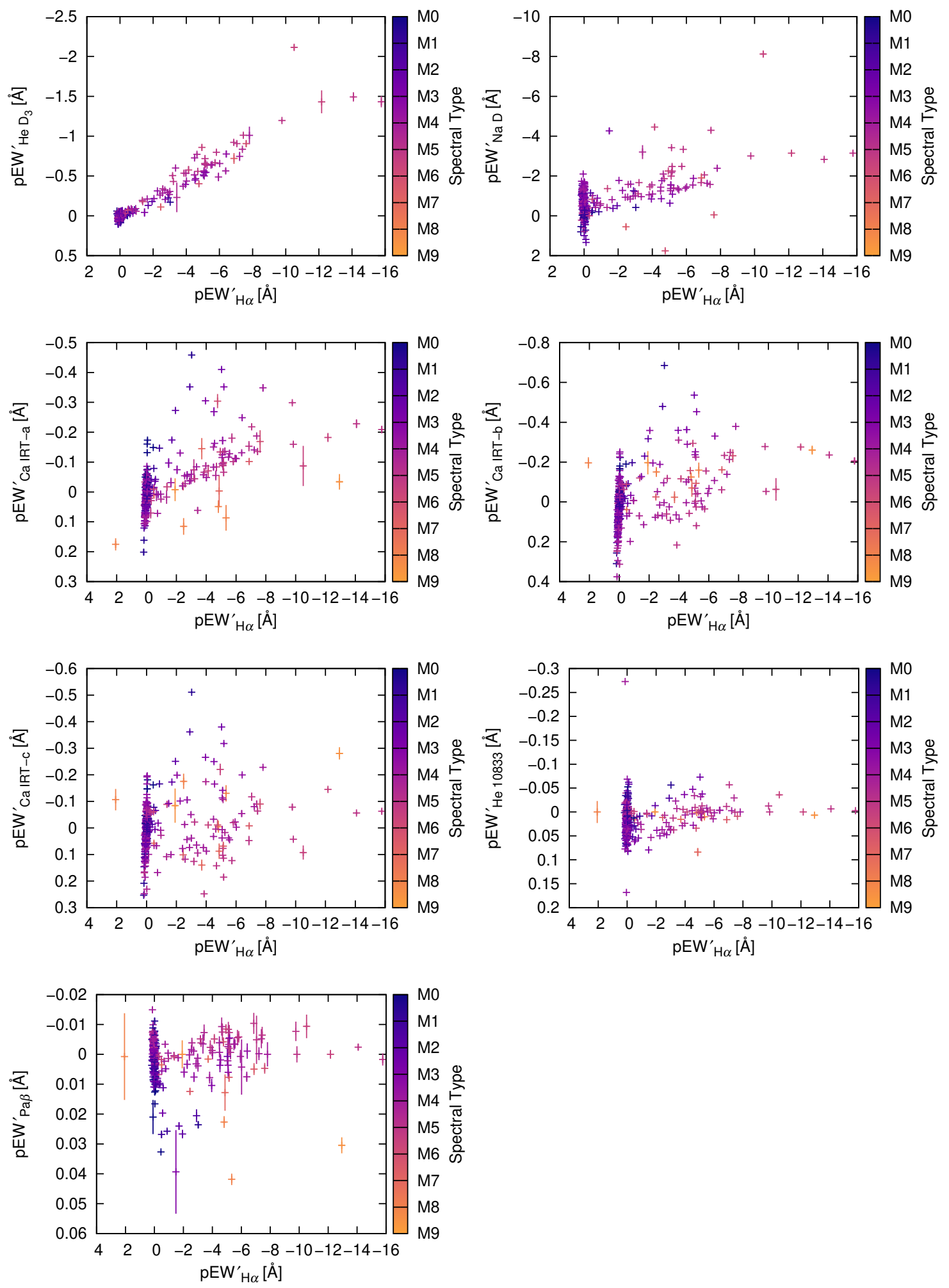

Figure 3.3.: Scatter plots of $\mathrm{pEW}_{\mathrm{H} \alpha}^{\prime}$ versus $\mathrm{pEW}^{\prime}$ of other spectral lines with a chromospheric component. Colours correspond to spectral subtypes. 

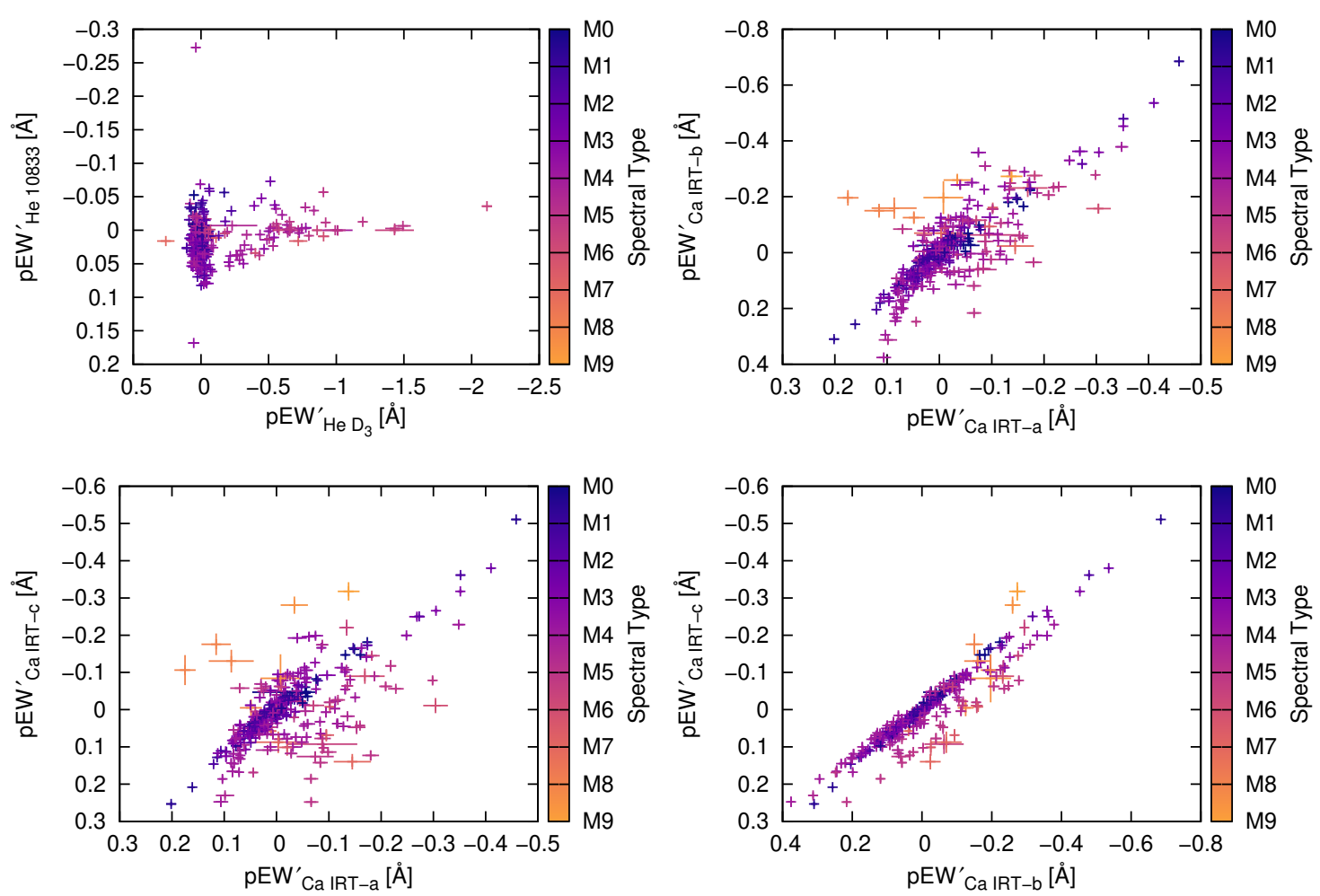

Figure 3.4.: Scatter plots of $\mathrm{pEW}_{\mathrm{He}_{\mathrm{D}}}^{\prime}$ versus $\mathrm{pEW}_{\mathrm{He}}^{\prime} 10833$ and of $\mathrm{pEW}_{\mathrm{Ca} \text { IRT-a }}^{\prime}, \mathrm{pEW}_{\mathrm{Ca} \text { IRT-b}}^{\prime}$, and $\mathrm{pEW}_{\mathrm{Ca} \text { IRT-c }}^{\prime}$ versus each other. Colours correspond to spectral subtypes.

visible, but most $\mathrm{H} \alpha$ active stars are of similar, mid-to-late subtypes, while the earliertype $\mathrm{H} \alpha$ active stars span only a small range of $\mathrm{pEW}_{\mathrm{H} \alpha}^{\prime}$ values and $\mathrm{pEW}_{\mathrm{He}_{3}}^{\prime}$ could not be measured for the latest subtypes.

In the scatter plot of $\mathrm{H} \alpha$ versus $\mathrm{Na} \mathrm{D}$, the $\mathrm{H} \alpha$ inactive stars form an elongated point cloud, as $\mathrm{pEW}_{\mathrm{H} \alpha}^{\prime} \approx 0 \AA$ for all of them, but $\mathrm{pEW}_{\mathrm{Na} \mathrm{D}}^{\prime}$ takes values between $-2.10 \AA$ and $1.34 \AA$. This is consistent with the spread of the $\mathrm{H} \alpha$ inactive stars in $\mathrm{Na} D$ described the previous section. By excluding the cloud of $\mathrm{H} \alpha$ inactive stars, the correlation coefficient increases from a weak $r_{S}=0.23$ to a strong $r_{S}=0.68$.

Similar to $\mathrm{H} \alpha$ versus $\mathrm{Na} \mathrm{D}$, the scatter plots of $\mathrm{H} \alpha$ versus the Ca IRT lines show the $\mathrm{H} \alpha$ inactive stars as an elongated point cloud. However, this cloud is slightly tilted and appears to increase the correlation coefficients because the majority of the sample stars is $\mathrm{H} \alpha$ inactive. For the $\mathrm{H} \alpha$ active stars only, the correlations coefficients are significantly weaker. Unlike in the previously described scatter plots, the slope of the $\mathrm{H} \alpha$ active stars appears steeper for earlier spectral subtypes. This is expected because the pseudo-continuum flux is temperature-dependent similar to Eq. 1.1.

The scatter plots of $\mathrm{H} \alpha$ versus $\mathrm{He} 10833$ and $\mathrm{Pa} \beta$ in Fig. 3.3, and of $\mathrm{He}_{3} \mathrm{D}_{3}$ versus He 10833 in Fig. 3.4 show no strong correlations between these indicators. Excess absorption in $\mathrm{He} 10833$ and $\mathrm{Pa} \beta$ is less common in stars with stronger excess emission in 
Table 3.2.: Same as Table 3.1, but for only the $\mathrm{H} \alpha$ active stars.

\begin{tabular}{lrrrrrrr}
\hline & $\mathrm{H} \alpha$ & $\mathrm{He} \mathrm{D}_{3}$ & Na D & Ca IRT-a & Ca IRT-b & Ca IRT-c & He 10833 \\
\hline $\mathrm{He} \mathrm{D}_{3}$ & $\mathbf{0 . 9 5}$ & $\ldots$ & & & & & \\
$\mathrm{Na} \mathrm{D}$ & $\mathbf{0 . 6 8}$ & $\mathbf{0 . 7 2}$ & $\ldots$ & & & & \\
Ca IRT-a & 0.56 & 0.58 & 0.26 & $\ldots$ & & & \\
Ca IRT-b & 0.32 & 0.26 & 0.17 & $\mathbf{0 . 6 2}$ & $\ldots$ & & \\
Ca IRT-c & 0.08 & 0.01 & -0.02 & 0.47 & $\mathbf{0 . 9 1}$ & $\ldots$ & \\
$\mathrm{He} 10833$ & 0.53 & 0.57 & 0.30 & 0.52 & 0.26 & 0.14 & $\ldots$ \\
$\mathrm{Pa} \beta$ & 0.41 & $\mathbf{0 . 6 4}$ & 0.55 & 0.11 & -0.21 & -0.36 & 0.43 \\
\hline
\end{tabular}

$\mathrm{H} \alpha$ and $\mathrm{He}_{3}$, leading to moderate correlation coefficients for the $\mathrm{H} \alpha$ active stars only. However, the strong excess absorption in $\mathrm{He} 10833$ and $\mathrm{Pa} \beta$ is only seen for earlier spectral subtypes and there is no correlation visible for mid-to-late type stars only.

In the remaining scatter plots in Fig. 3.4, strong correlations between the Ca IRT lines are visible. However, the correlation coefficients decrease if the $\mathrm{H} \alpha$ inactive stars are excluded. Therefore, the strong correlation is likely caused by a correlation of the deviations in the line profiles of the photospheric components. The very late-type stars appear as outliers because the M6.0 reference star is used for the subtraction, but the pseudo-continua around the three Ca IRT lines behave differently at later types.

\subsection{Temporal variability}

While the previous sections explored the typical $\mathrm{pEW}^{\prime}$ values of each star as derived from the co-added template spectra, I now investigate how the $\mathrm{pEW}^{\prime}$ values from individual spectra vary for each star. Occasional strong flares can cause drastic short-term variations that lead to a heavy-tailed non-normal distribution of a star's $\mathrm{pEW}^{\prime}$ values. Unlike the variance or standard deviation, a difference between percentiles does not assume a normal distribution. Therefore, I defined the absolute variation of each indicators as the difference between the 20th and 80th percentiles calculated using the quantile estimator by Harrell \& Davis (1982). This estimator does not assume a specific distribution and also provides the errors of the percentiles. The error of the absolute variation is the root sum of squares of the percentile errors. Division of the absolute variation by the $\mathrm{pEW}^{\prime}$ from the co-added template spectrum yields the relative variation. For each chromospheric indicator, this relative variation of each $\mathrm{H} \alpha$ active star is shown as a function of the normalised $\mathrm{H} \alpha$ luminosity in Fig. 3.5. $\mathrm{H} \alpha$ active stars can have $\mathrm{pEW}^{\prime}$ values close to $0 \AA$ in the other indicators, leading to very large relative variations with large error bars. For reasons of clarity, I excluded four stars with a relative $\mathrm{He}_{3}$ variation larger than 3 , and three stars, four stars, five stars, and six stars with relative Na D, Ca IRT-a, Ca IRT-b, and Ca IRT-c variations larger than 5 from Fig. 3.5. The relative $\mathrm{He} 10833$ and $\mathrm{Pa} \beta$ variations are not shown. Although measurements from spectra with telluric contamination are excluded as 

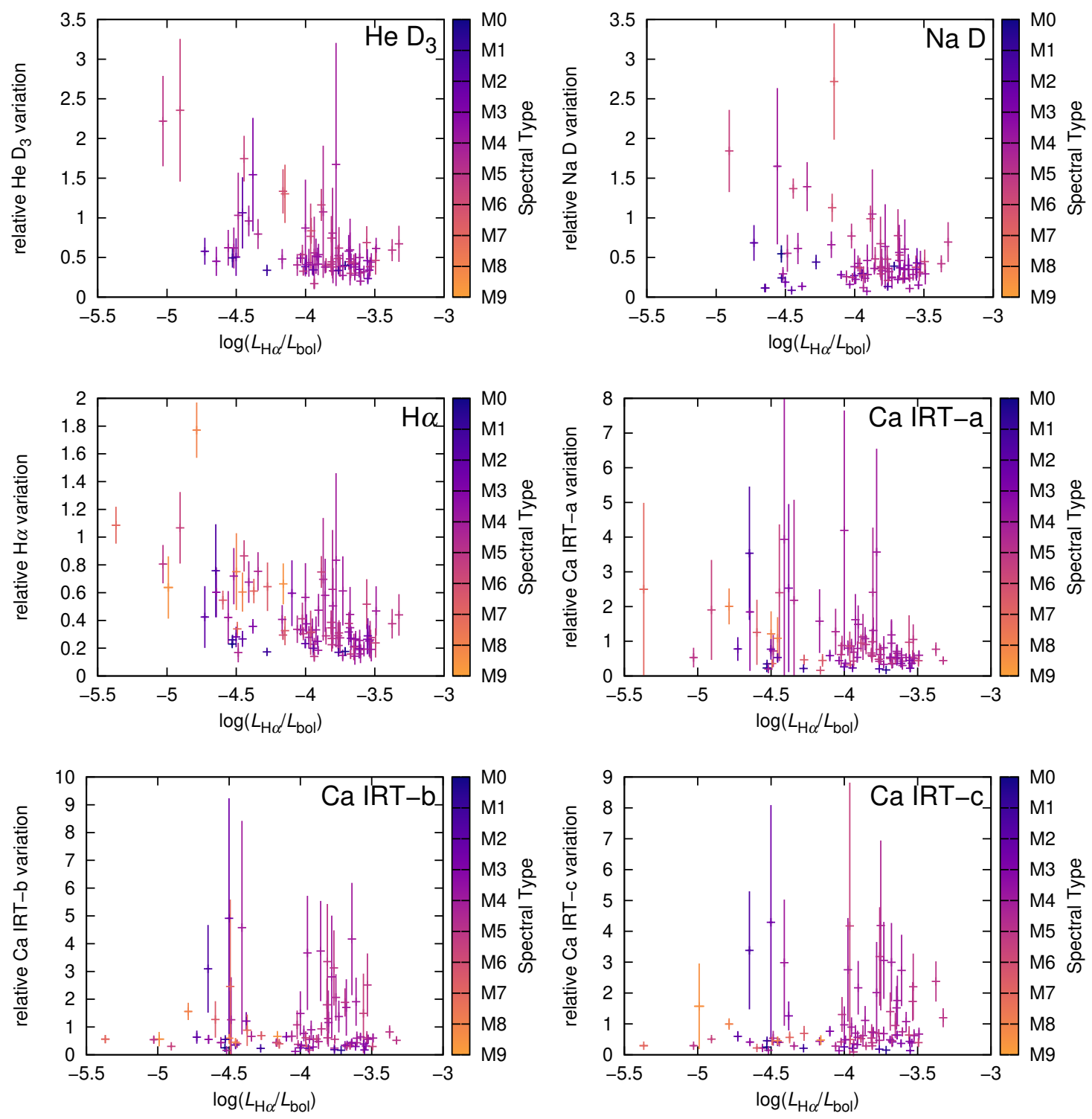

Figure 3.5.: Relative $\mathrm{pEW}^{\prime}$ variations of $\mathrm{H} \alpha$ active stars as functions of normalised $\mathrm{H} \alpha$ luminosity. Colours correspond to spectral subtypes. 


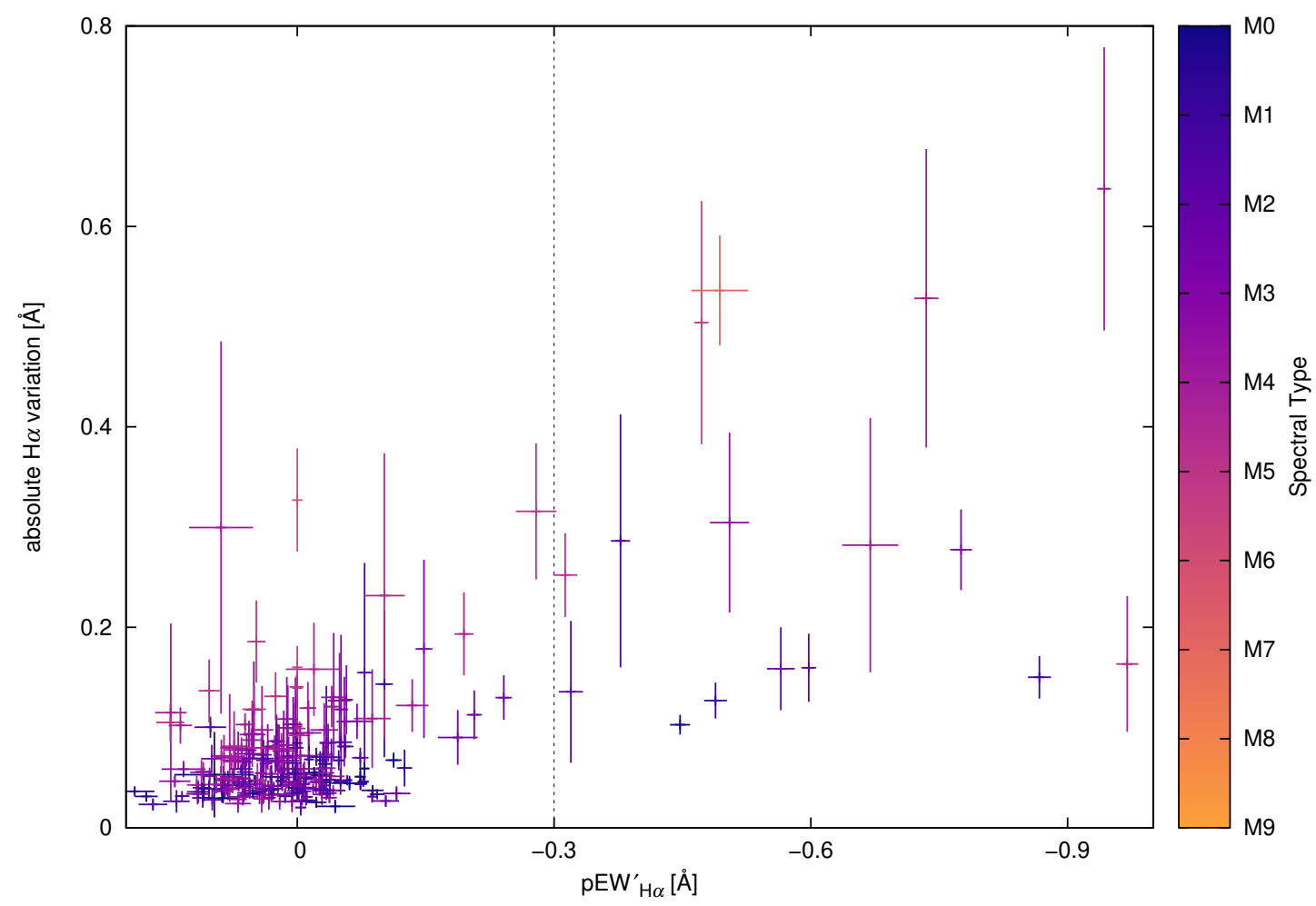

Figure 3.6.: Absolute $\mathrm{H} \alpha$ variation as a function of $\mathrm{pEW}_{\mathrm{H} \alpha}^{\prime}$ for $\mathrm{H} \alpha$ inactive and low-active stars. The dashed black line marks the activity threshold at $\mathrm{pEW}_{\mathrm{H} \alpha}^{\prime}=-0.3 \AA$. Colours correspond to spectral subtypes.

described in Sect. 2.2, even more stars show large variations and error bars in these two indicators. I again calculated Spearman's rank coefficient $r_{S}$ to quantify the correlations between the relative variations and the normalised $\mathrm{H} \alpha$ luminosity. The stars excluded from Fig. 3.5 are included in the calculation of the correlation coefficients.

With a moderate correlation coefficient of $r_{S}=-0.50$, the relative $\mathrm{He}_{3}$ variation shows the strongest anti-correlation with the normalised $\mathrm{H} \alpha$ luminosity. At $r_{S}=-0.48$, the relative $\mathrm{H} \alpha$ variation is also moderately anti-correlated with the normalised $\mathrm{H} \alpha$ luminosity, and includes very late-type stars for which $\mathrm{He}_{3}$ could not be measured. The relative $\mathrm{Na} \mathrm{D}$ and $\mathrm{Ca}$ IRT-a variations are weakly anti-correlated with the normalised $\mathrm{H} \alpha$ luminosity with $r_{S}=-0.15$ and $r_{S}=-0.34$, whereas the relative Ca IRT-b, Ca IRT-c, He 10833, and $\mathrm{Pa} \beta$ variations show no correlation or weak positive correlations with $r_{S}=0.02$, $r_{S}=0.20, r_{S}=0.32$, and $r_{S}=0.22$.

For the $\mathrm{H} \alpha$ inactive stars, I show the absolute $\mathrm{H} \alpha$ variation as a function of $\mathrm{pEW}_{\mathrm{H} \alpha}^{\prime}$ in Fig. 3.6. Not shown is J19255+096 with $\mathrm{pEW}_{\mathrm{H} \alpha}^{\prime}=(2.07 \pm 0.08) \AA$ and an absolute $\mathrm{H} \alpha$ variation of $(3.2 \pm 0.5) \AA$. The $\mathrm{H} \alpha$ inactive stars are concentrated around $0 \AA$ in $\mathrm{pEW}_{\mathrm{H} \alpha}^{\prime}$ and have a median absolute $\mathrm{H} \alpha$ variation of $0.053 \AA$. In general, the absolute variations are larger for later spectral subtypes because a lower SNR leads to higher statistical fluc- 
tuations. The $\mathrm{H} \alpha$ active stars with an excess emission barely above the activity threshold at $\mathrm{pEW}_{\mathrm{H} \alpha}^{\prime}=-0.3 \AA$ show generally larger absolute variations than $\mathrm{H} \alpha$ inactive stars of the same spectral subtype. This reaffirms the choice of an activity threshold close to the boundary of the cloud of $\mathrm{H} \alpha$ inactive stars.

\subsection{Impact of reference stars}

All results presented in the previous sections were derived using the stars with the longest rotation period for each spectral subtype as reference stars for the spectral subtraction. As outlined in Sect. 2.2.1, I also measured all $\mathrm{pEW}^{\prime}$ values using the stars with the smallest variation of the $\mathrm{H} \alpha \mathrm{MNI}$ for each subtype as reference stars. For each $\mathrm{pEW}$ ' measurement, I calculated the difference $\Delta_{\text {rot }}^{\mathrm{MNI}}$ between the result using the stars with the smallest $\mathrm{H} \alpha$ MNI variation and the result using the stars with the longest rotation period as reference stars. The differences $\Delta_{\text {rot }}^{\mathrm{MNI}}$ from the co-added template spectra for all chromospheric indicators are shown in Fig. 3.7 as a function of the $\mathrm{pEW}^{\prime}$ measured using the reference stars with the longest rotation period. Error bars are derived from the uncertainties of both $\mathrm{pEW}^{\prime}$ measurements via propagation of uncertainty. Spectral subtypes later than M5.0 are not shown because both sets of reference stars include the same stars for the latest spectral subtypes, and hence there are no differences.

A different reference star can introduce a constant offset and a linear trend for all stars of the same spectral subtype. Both effects are seen for some subtypes in some indicators: While constant offsets are most clearly seen for Na D and the Ca IRT lines, which all have a strong photospheric component, and for He 10833, the most obvious linear trend is seen for the M4.0 stars in $\mathrm{H} \alpha$, where $\Delta_{\text {rot }}^{\mathrm{MNI}}$ decreases from $-0.09 \AA$ for the H $\alpha$ inactive stars at $\mathrm{pEW}_{\mathrm{H} \alpha}^{\prime} \approx 0 \AA$ to $-0.15 \AA$ for the star with the strongest excess emission at $\mathrm{pEW}_{\mathrm{H} \alpha}^{\prime}=-7.81 \pm 0.06 \AA$. However, most linear trends are insignificant compared to the measurement uncertainties. In general, for $\mathrm{He}_{3}$ and $\mathrm{H} \alpha$, the differences are on the order of the spread of the $\mathrm{H} \alpha$ inactive stars, but become insignificant with increasing excess emission. On the other hand, the differences in the other indicators are significant also for $\mathrm{H} \alpha$ active stars, but they are dominated by only a constant offset.

I also calculated the $\mathrm{pEW}^{\prime}$ variations of each star as defined in the previous section using the $\mathrm{pEW}^{\prime}$ measurements derived with both sets of reference stars. In Fig. 3.8, the absolute variations derived from the $\mathrm{pEW}^{\prime}$ measurements using the reference stars with the smallest $\mathrm{H} \alpha$ MNI variations are shown as a function of the absolute variations derived from the $\mathrm{pEW}^{\prime}$ measurements using the reference stars with the longest rotation periods. As the variation is the difference between two $\mathrm{pEW}^{\prime}$ measurements of the same star, any constant offset introduced by the choice of the reference star is eliminated. Therefore, the absolute variations derived with both sets of reference stars are in good agreement with each other. 

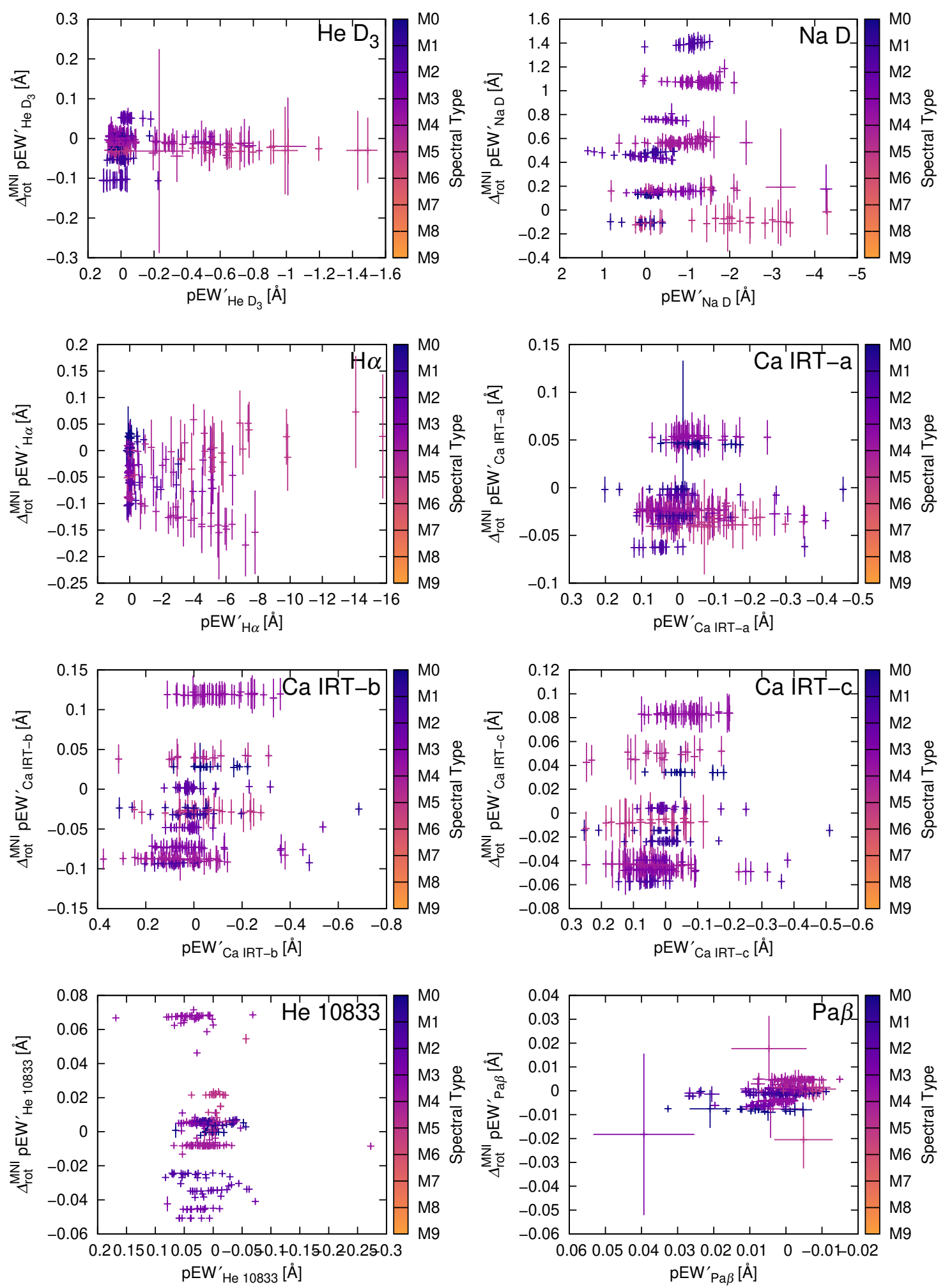

Figure 3.7.: Differences between $p E W^{\prime}$ values calculated with different sets of reference stars as a function of $\mathrm{pEW}^{\prime}$ values from the previous sections. Colours correspond to spectral subtypes. 

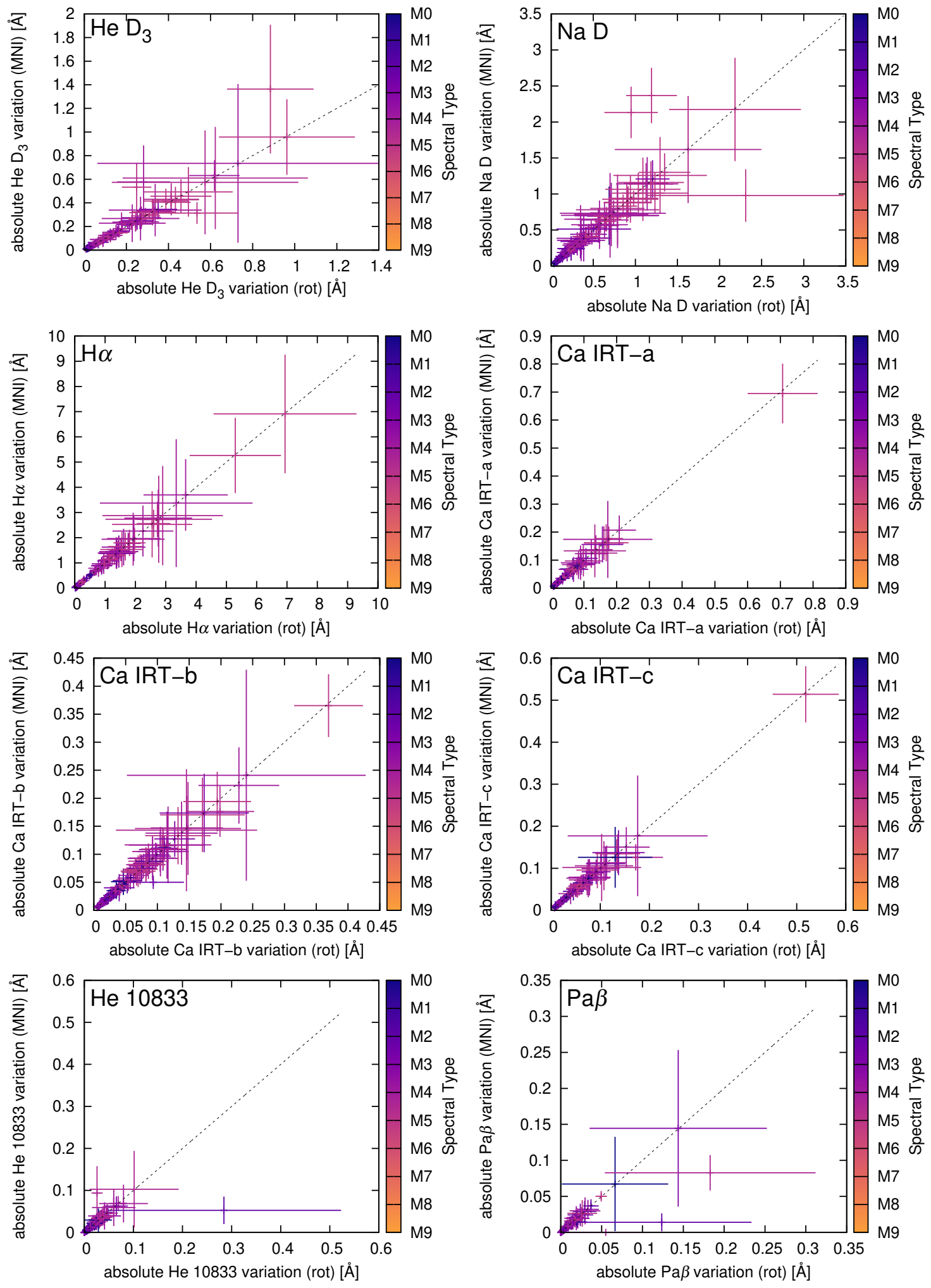

Figure 3.8.: Scatter plots of $\mathrm{pEW}^{\prime}$ variations calculated with different sets of reference stars. Dashed black lines mark the one-to-one relation. Colours correspond to spectral subtypes. 


\subsection{Discussion}

In Fig. 3.9, I compare the $\mathrm{pEW}^{\prime}$ values presented in this thesis with the earlier results from Schöfer et al. (2019) that do not include observations after July 2018. While the results generally agree with each other for all chromospheric lines, a few outliers from the oneto-one relation are visible. The outliers are mostly later-type stars, so a larger statistical fluctuation because of a lower SNR is expected, particularly for $\mathrm{He}_{3}$ and $\mathrm{Na} \mathrm{D}$. In $\mathrm{H} \alpha$, the two most significant outliers are J10584-107 and J19255+096. The former star is also the only significant outlier in the Ca IRT lines. In this case, the co-added template spectrum used in the earlier work included a mislabelled spectrum of a nearby $\mathrm{K}$ dwarf and therefore showed erroneously strong absorption. The latter star already appeared as an outlier previously as it is the only star later than M6.0 that was classified as $\mathrm{H} \alpha$ active. Although the number of observations increased significantly from 36 to 111, the co-added template spectrum is very noisy around $\mathrm{H} \alpha$. At spectral type M8.0, the flux is insufficient in this wavelength region for a robust measurement. In He 10833, a group of outliers with large error bars in the earlier results is visible. The co-added template spectra of these stars were bad because of a bad background subtraction for some observations, but were fixed after July 2018. In $\mathrm{Pa} \beta$, most outliers also have large error bars and are still consistent with the one-to-one relation.

A comparison of the $\mathrm{pEW}_{\mathrm{H} \alpha}^{\prime}$ values with $\mathrm{pEW}_{\mathrm{H} \alpha}$ values compiled by Jeffers et al. (2018) and derived without the spectral subtraction is shown in Fig. 3.10. Despite the different methods, the results generally agree with each other. There are more stars below the oneto-one relation than above, so the subtraction method leads to more negative values. This is expected, as the spectral subtraction removes photospheric absorption lines in the line window.

I now compare the normalised $\mathrm{H} \alpha$ luminosity to independent activity indicators. While the catalogue of $\mathrm{Ca} H \& \mathrm{~K}$ measurements by Boro Saikia et al. (2018) contains $R_{\mathrm{HK}}^{\prime}$ values for 28 sample stars, only one of these stars (J18075-159) is $\mathrm{H} \alpha$ active. With $\log R_{\mathrm{HK}}^{\prime}=$ -4.345 , it shows a higher normalised chromospheric $\mathrm{Ca} \mathrm{H} \& \mathrm{~K}$ flux than the $27 \mathrm{H} \alpha$ inactive stars with $-5.434<\log R_{\mathrm{HK}}^{\prime}<-4.420$. The top left panel of Fig. 3.11 shows the projected rotational velocities $v \sin i$ as tabulated in Table A.1 as a function of $\log \left(L_{\mathrm{H} \alpha} / L_{\mathrm{bol}}\right)$. As expected from the correlation between chromospheric activity and rotation, the stars with a higher normalised $\mathrm{H} \alpha$ luminosity are the fastest rotators. For very late-type stars, the normalised $\mathrm{H} \alpha$ luminosity is lower than for earlier-type stars with the same $v \sin i$. It is possible that $\log \left(L_{\mathrm{H} \alpha} / L_{\mathrm{bol}}\right)$ is systematically lower for very late spectral subtypes, because less absorption in the $\mathrm{H} \alpha$ line window is removed by the spectral subtraction, as the reference star is of an earlier spectral subtype. In the top right panel of Fig. 3.11, the rotation periods $P_{\text {rot }}$ are shown as a function of $\log \left(L_{\mathrm{H} \alpha} / L_{\mathrm{bol}}\right)$. The result is similar to the result from Jeffers et al. (2018) shown in Fig. 1.13 and also to the previous panel, although the inclination angle of the stellar rotation axis is removed as a factor. Stars with a higher normalised $\mathrm{H} \alpha$ luminosity have shorter rotation periods and are thus faster rotators. Shulyak et al. (2019) measured magnetic fields of $29 \mathrm{H} \alpha$ active sample stars. 

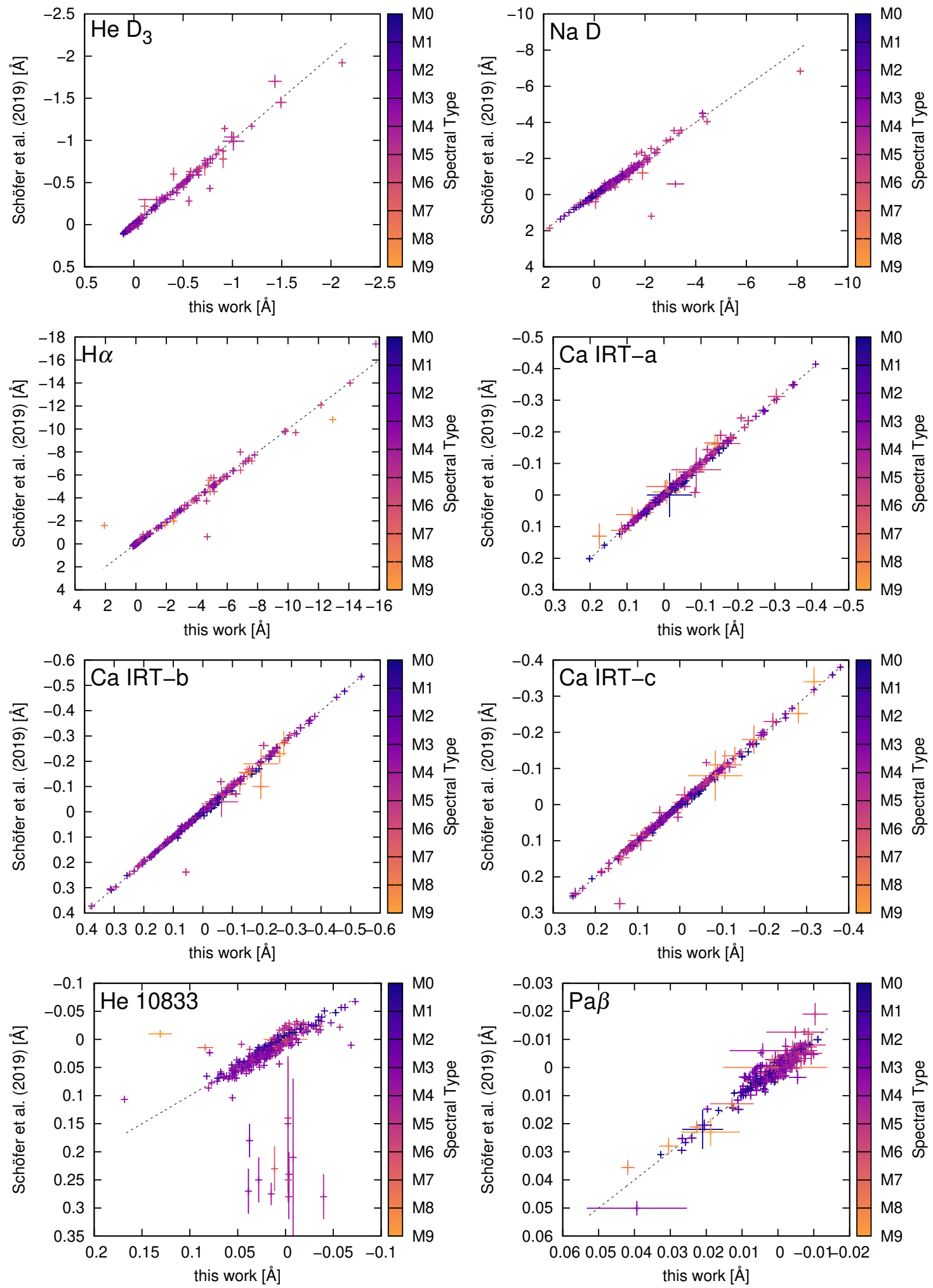

Figure 3.9.: Comparison of $\mathrm{pEW}^{\prime}$ values derived in this thesis and in Schöfer et al. (2019). Dashed black lines mark the one-to-one relation. Colours correspond to spectral subtypes. 


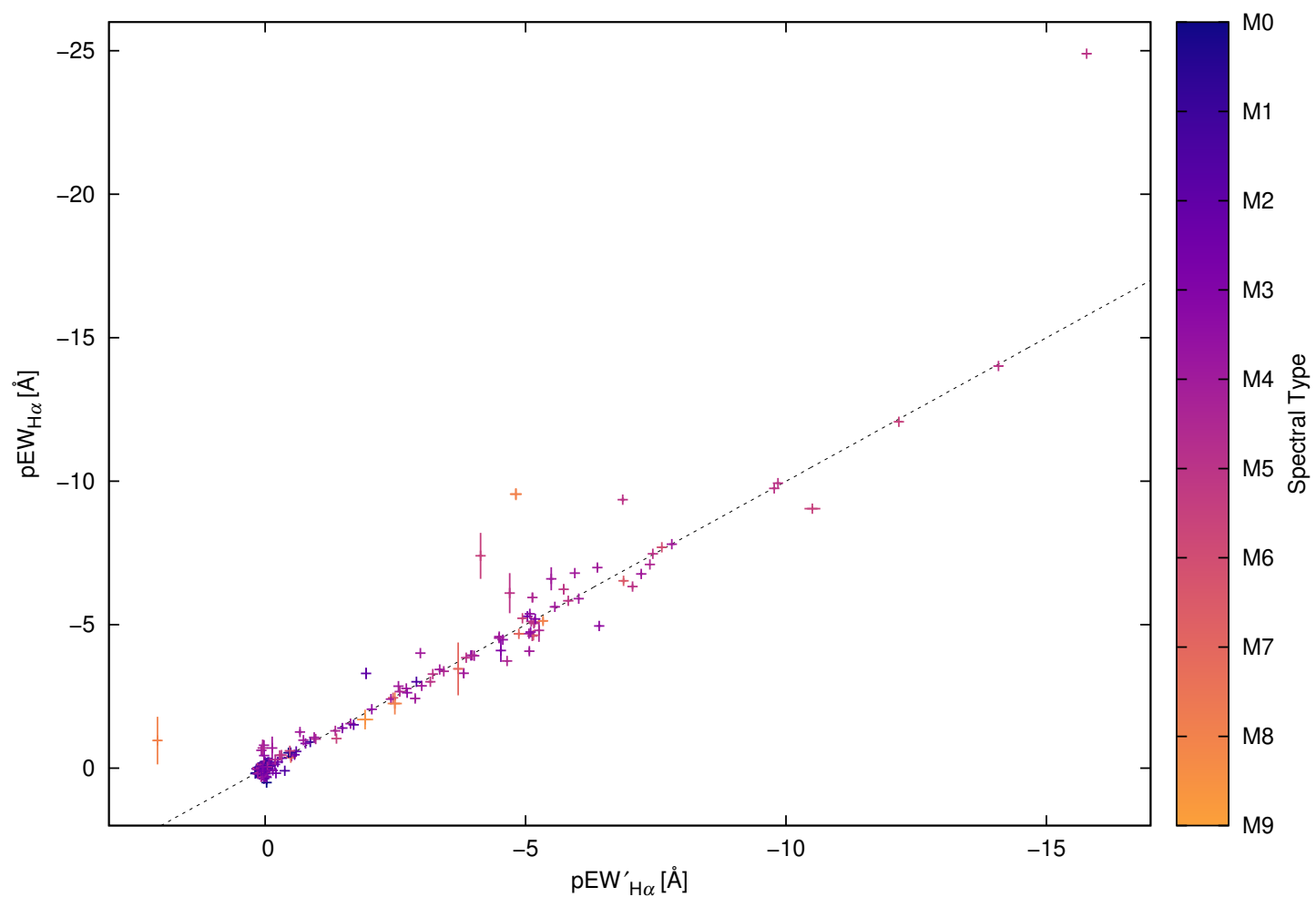

Figure 3.10.: Comparison of $\mathrm{pEW}^{\prime}$ values with $\mathrm{pEW}$ values reported by Jeffers et al. (2018). The dashed black line marks the one-to-one relation. Colours correspond to spectral subtypes. 

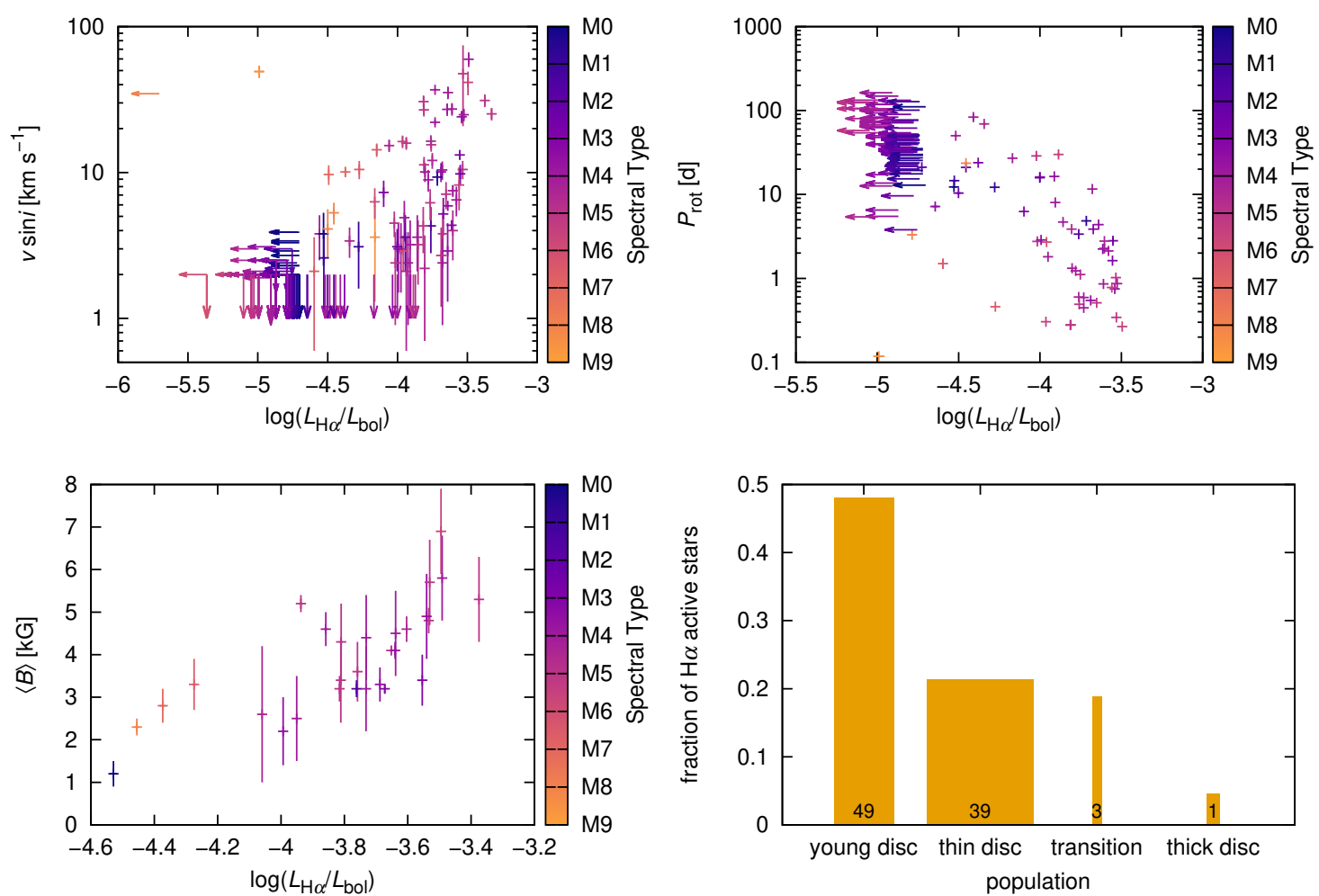

Figure 3.11.: Top left: projected rotational velocity $v \sin i$ as given in Table A.1 as a function of normalised $\mathrm{H} \alpha$ luminosity. Arrows denote lower limits, colours correspond to spectral subtypes. Top right: rotation period $P_{\text {rot }}$ as given in Table A.1 as a function of normalised $\mathrm{H} \alpha$ luminosity. Arrows denote lower limits, colours correspond to spectral subtypes. Bottom left: total magnetic field strength $\langle B\rangle$ derived by Shulyak et al. (2019) as a function of normalised $\mathrm{H} \alpha$ luminosity. Colours correspond to spectral subtypes. Bottom right: fraction of $\mathrm{H} \alpha$ active stars in stellar populations as given by Cortés-Contreras et al. (in prep.). Box width is proportional to the number of stars in each population, so the area of each bar corresponds to the specified number of $\mathrm{H} \alpha$ active stars in that population.

As shown in the bottom left panel of Fig. 3.11, the normalised $\mathrm{H} \alpha$ luminosity is strongly correlated with the total magnetic field strength $\langle B\rangle$, consistent with the assumption that the chromospheric emission is driven by magnetic heating. The bottom right panel of Fig. 3.11 shows the fraction of $\mathrm{H} \alpha$ active stars for the different kinematic stellar populations identified by Cortés-Contreras et al. (in prep.). While $48 \%$ of the stars in the young disc population are $\mathrm{H} \alpha$ active, the fraction decreases to $21 \%$ for the remaining thin disc population, 3 of 16 stars in transition region between the thin and the thick disc, and only 1 of 22 stars in the thick disc population. As these populations are a rough indicator of the age of the stars, the decreasing fraction of $\mathrm{H} \alpha$ active stars is consistent with the correlation of chromospheric activity and age.

With an overall tally of $95 \mathrm{H} \alpha$ active stars, one star less has been classified as $\mathrm{H} \alpha$ active than in Schöfer et al. (2019), although 6 new stars were included in the sample. In addition 
to the already discussed very late-type star J19255+096, $\mathrm{pEW}_{\mathrm{H} \alpha}^{\prime}$ could not be measured in the co-added template spectrum of the previously $\mathrm{H} \alpha$ active star J06318+414 because the spectral order containing $\mathrm{H} \alpha$ was excluded from the spectrum, and the $\mathrm{pEW}_{\mathrm{H} \alpha}^{\prime}$ of J06594+193 changed from $(-0.428 \pm 0.026) \AA$ to $(-0.279 \pm 0.024) \AA$ below the activity threshold. On the other hand, the $\mathrm{pEW}_{\mathrm{H} \alpha}^{\prime}$ of J03217-066 changed from $(-0.274 \pm 0.022) \AA$ to $(-0.320 \pm 0.015) \AA$ above the activity threshold, and the early-type $\mathrm{H} \alpha$ active star J20451-313 was added. Therefore, the classification of $\mathrm{H} \alpha$ active stars is relatively stable. The distribution of the $\mathrm{H} \alpha$ active stars among spectral subtypes is similar to the distribution found in previous studies of nearby $M$ dwarfs (e.g. Reiners et al. 2012; West et al. 2015; Jeffers et al. 2018) that was discussed in Sect. 1.4.2, and a drastic increase in the fraction of $\mathrm{H} \alpha$ active stars occurs between spectral subtypes M3.5 and M4.0, where stars no longer have a radiative core and become fully convective, as described in Sect. 1.2.4.

The correlations presented in Sect. 3.2 are as expected from the distribution of the $\mathrm{pEW}^{\prime}$ values among the spectral subtypes described in Sect. 3.1. $\mathrm{pEW}_{\mathrm{H} \alpha}^{\prime}$ and $\mathrm{pEW}_{\mathrm{He}_{3}}^{\prime}$ show the most similar distribution and this translates into a very strong correlation. A linear correlation between $\mathrm{H} \alpha$ and $\mathrm{He} \mathrm{D}_{3}$ was previously reported by Gizis et al. (2002) and suggests that these lines are formed in the same region of the chromosphere. In $\mathrm{Na} \mathrm{D}$ and the $\mathrm{Ca}$ IRT lines, the $\mathrm{H} \alpha$ inactive stars of each spectral subtype are spread around the respective reference stars. As the spectral subtypes are a coarse classification that groups stars with a range of different effective temperatures, metallicities, and surface gravity together, the differences in these parameters cause different line shapes of the photospheric component (e.g. Mallik 1997; Schlieder et al. 2012), and the subtraction of a reference star spectrum can only remove photospheric lines with the same shape. While the correlation between $\mathrm{Na} \mathrm{D}$ and $\mathrm{H} \alpha$ is stronger for the $\mathrm{H} \alpha$ active stars only, the correlations between the Ca IRT lines and $\mathrm{H} \alpha$ become weaker when the $\mathrm{H} \alpha$ inactive stars are excluded. This is likely an effect of the different pseudo-continuum flux ratios for different spectral subtypes. He 10833 and $\mathrm{Pa} \beta$ show distributions per spectral type that are very different from $\mathrm{H} \alpha$ and are therefore weaker correlated with $\mathrm{H} \alpha$. The narrow line windows for these two lines increase the chance that not the full excess absorption or emission is measured, as either the true line may be broader or an imprecise Doppler shift correction may lead to the line not being centred in the line window. However, Fuhrmeister et al. (2019a) measured the pEW of the He 10833 line by fitting a Voigt profile and found a similar spectral type distribution, but with an additional spectral-type dependent trend that is removed by the spectral subtraction in the $\mathrm{pEW}^{\prime}$ measurements. A broader line window would cause even more measurements from individual spectra to be contaminated by the nearby telluric lines. CARMENES spectra with telluric absorption lines removed are available (Nagel et al. submitted), but there is also an airglow line close to He 10833 that is not removed in those spectra.

In $\mathrm{He} \mathrm{D}_{3}$ and $\mathrm{H} \alpha$, the relative variations decrease with increasing activity as measured by the normalised $\mathrm{H} \alpha$ luminosity. Bell et al. (2012) found the same behaviour for $\mathrm{H} \alpha$. If a star has many active regions on its surface, then the contribution of a single active region that appears or disappears to the global activity level is negligible. Therefore, it 
is plausible that the relative variation is anti-correlated with the activity level. In $\mathrm{Na} \mathrm{D}$ and the Ca IRT line, the anti-correlation is not clearly seen. While the bias introduced by the spectral subtraction is removed in the calculation of the absolute variation, it is re-introduced by the division to obtain the relative variation. The relative variations of He 10833 and $\mathrm{Pa} \beta$ are not useful, as the measured $\mathrm{pEW}^{\prime}$ values can be very close to $0 \AA$ also for $\mathrm{H} \alpha$ active stars and a division by a value close to zero yields very large results.

As shown in Sect. 3.4, a different choice of the reference star introduces mostly a constant offset. For the lines with a photospheric absorption component, this offset can be significant. However, there is no easy way to remove the offset. A more fine-grained grouping of the stars leads to a larger number of groups and the $\mathrm{pEW}^{\prime}$ values from different groups cannot be directly compared. An alternative approach is the subtraction of a synthetic model spectrum instead of a reference star spectrum. However, while high-resolution PHOENIX models are available for a large parameter space (Husser et al. 2013), the line lists for the photospheric absorption bands may not be sufficiently accurate to reproduce the absorption lines that should be removed by the spectral subtraction. In addition, results derived using different model spectra would also need to be normalised before they can be compared with each other. 


\section{Photospheric indicators}

The photosphere is the lowest layer of a stellar atmosphere, but dominates the light that reaches the observer, as described in Sect. 1.2.4. Strong absorption bands by titanium oxide and other molecules in the photosphere are the characteristic feature in M dwarf spectra.

In this chapter, I present and analyse the indices described in Sect. 2.3 that measure the strength of these absorption band in the M dwarfs of the CARMENES sample, starting with the spectral-type dependence as an overview in Sect. 4.1. While TiO 7050, TiO 8430, VO 7436, and VO 7942 were also presented in Schöfer et al. (2019), CaH 2*, CaH 3*, $\mathrm{TiO} 8860$, and FeH WFB are new indices, and I include additional observations after July 2018 in this thesis. In Sect. 4.2, I explore correlations both among the band indices and with chromospheric activity as measured by the normalised $\mathrm{H} \alpha$ luminosity. This leads to the definition of a new activity indicator. Sect. 4.3 quantitatively investigates the temporal variations of the band indices. In Sect. 4.4, I compare the results with previous results and discuss them.

\subsection{Sample overview}

The photospheric absorption band indices derived from the co-added template spectra for each star are shown in Fig. 4.1 as a function of spectral type. H $\alpha$ active stars are colourcoded by their normalised $\mathrm{H} \alpha$ luminosity, whereas $\mathrm{H} \alpha$ inactive stars and the M9.0 star with no valid normalised $\mathrm{H} \alpha$ luminosity measurement are shown in black.

All indices show a clear spectral-type dependence. While $\mathrm{CaH} 2^{*}, \mathrm{CaH} 3^{*}$, and $\mathrm{TiO} 7050$ decrease linearly with the spectral type up to M6.0, the other indicators tend to decrease stronger with increasing spectral type. At very late types later than M6.0, the indices appear to be constant or increase again, but with only four or less stars per subtype, the sample is too sparse to detect if these are outliers. The $\mathrm{CaH} 3^{*}$, TiO 7050, TiO 8430, and FeH WFB index values of $\mathrm{H} \alpha$ active stars tend to be lower than the values of $\mathrm{H} \alpha$ inactive stars of the same spectral subtype. The opposite is true for $\mathrm{CaH} 2^{*}$, whereas VO 7436, VO 7942, and TiO 8860 show no clear trends for $\mathrm{H} \alpha$ active stars. 

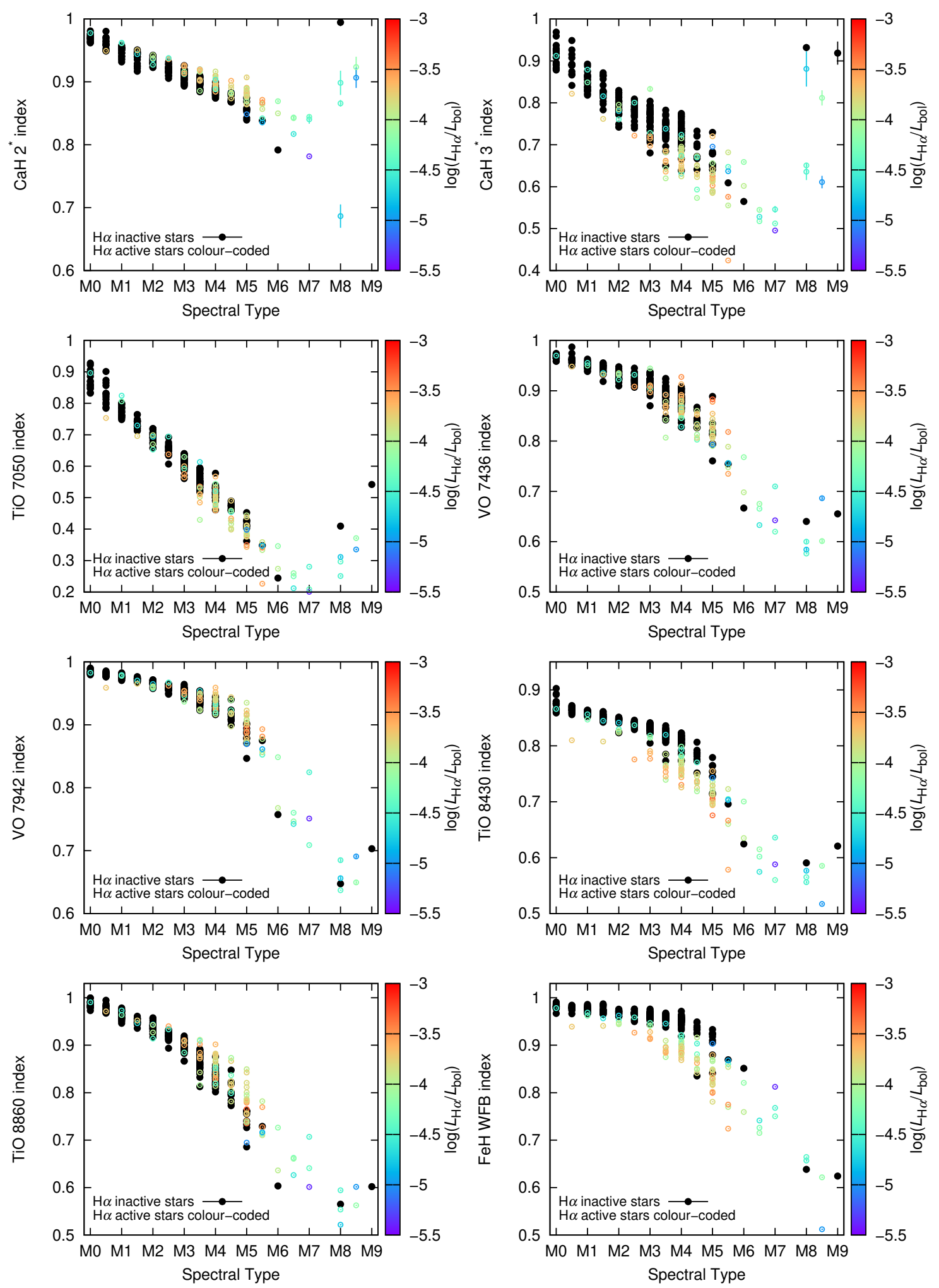

Figure 4.1.: Photospheric absorption band indices as a function of spectral type. H $\alpha$ inactive stars are shown in black, $\mathrm{H} \alpha$ active stars are colour-coded by normalised $\mathrm{H} \alpha$ luminosity. 
Table 4.1.: Correlation table of photospheric absorption band indices using Spearman's rank coefficient $r_{S}$. All correlations are strong with $r_{S}>0.60$ and therefore highlighted in boldface.

\begin{tabular}{lrrrrrrr}
\hline & $\mathrm{CaH}^{*}$ & $\mathrm{CaH}^{*}$ & $\mathrm{TiO} 7050$ & $\mathrm{VO} 7436$ & $\mathrm{VO} 7942$ & $\mathrm{TiO} 8430$ & $\mathrm{TiO} 8860$ \\
\hline $\mathrm{CaH}^{*}$ & $\mathbf{0 . 8 1}$ & $\ldots$ & & & & & \\
$\mathrm{TiO} 7050$ & $\mathbf{0 . 9 2}$ & $\mathbf{0 . 8 9}$ & $\ldots$ & & & & \\
$\mathrm{VO} 7436$ & $\mathbf{0 . 8 6}$ & $\mathbf{0 . 8 9}$ & $\mathbf{0 . 9 6}$ & $\ldots$ & & & \\
$\mathrm{VO} 7942$ & $\mathbf{0 . 9 3}$ & $\mathbf{0 . 8 1}$ & $\mathbf{0 . 9 7}$ & $\mathbf{0 . 9 2}$ & $\ldots$ & & \\
$\mathrm{TiO} 8430$ & $\mathbf{0 . 8 4}$ & $\mathbf{0 . 9 0}$ & $\mathbf{0 . 9 6}$ & $\mathbf{0 . 9 5}$ & $\mathbf{0 . 9 2}$ & $\ldots$ & \\
$\mathrm{TiO} 8860$ & $\mathbf{0 . 9 4}$ & $\mathbf{0 . 8 1}$ & $\mathbf{0 . 9 7}$ & $\mathbf{0 . 9 2}$ & $\mathbf{0 . 9 9}$ & $\mathbf{0 . 9 1}$ & $\ldots$ \\
FeH WFB & $\mathbf{0 . 7 4}$ & $\mathbf{0 . 8 7}$ & $\mathbf{0 . 9 0}$ & $\mathbf{0 . 9 1}$ & $\mathbf{0 . 8 5}$ & $\mathbf{0 . 9 6}$ & $\mathbf{0 . 8 4}$ \\
\hline
\end{tabular}

\subsection{Correlations}

\subsubsection{Correlations among band indices}

The similar spectral-type dependence of all photospheric band indices suggests that the indices are strongly correlated with each other. Using the same colour-coding for $\mathrm{H} \alpha$ active and $\mathrm{H} \alpha$ inactive stars as in the previous section, Fig. 4.2 shows scatter plots of the TiO 7050 index versus all other indices, and Fig. 4.3 shows scatter plots of the two $\mathrm{CaH}$ band indices and of the two VO band indices. A correlation table with Spearman's rank coefficients $r_{S}$ is given in Table 4.1.

All photospheric absorption band indices are strongly correlated with each other, with correlation coefficients ranging from $r_{S}=0.74$ for $\mathrm{CaH} 2^{*}$ versus $\mathrm{FeH}$ WFB to $r_{S}=0.99$ for VO 7942 versus $\mathrm{TiO} 8860$. The scatter plots of $\mathrm{TiO} 7050$ versus $\mathrm{CaH} 2^{*}$, VO 7942, $\mathrm{TiO} 8430$, TiO 8860, and FeH WFB in Fig. 4.2 all show the $\mathrm{H} \alpha$ inactive stars located on a narrow line, while the $\mathrm{H} \alpha$ active stars are located in general either above the line of $\mathrm{H} \alpha$ inactive stars ( $\mathrm{TiO} 7050$ versus $\mathrm{CaH} \mathrm{2*}$, VO 7942, and $\mathrm{TiO} 8860$ ) or below that line ( $\mathrm{TiO} 7050$ versus $\mathrm{TiO} 8430$ and $\mathrm{FeH}$ WFB). This effect is more pronounced at lower index values that correspond to later spectral subtypes. In the scatter plots of $\mathrm{TiO} 7050$ versus $\mathrm{CaH} \mathrm{3}{ }^{*}$ shown in Fig. 4.2 and $\mathrm{CaH} \mathrm{2*}$ versus $\mathrm{CaH} 3^{*}$ shown in Fig. 4.3, the $\mathrm{H} \alpha$ inactive stars form a cloud rather than a line, and the $\mathrm{H} \alpha$ active stars are located at the bottom of that cloud. In contrast, the $\mathrm{H} \alpha$ inactive and $\mathrm{H} \alpha$ active stars are not separated in the scatter plots of TiO 7050 versus VO 7436 in Fig. 4.2 and VO 7436 versus VO 7942 in Fig. 4.3. As the monotonic decrease of the index values stops at different spectral subtypes for different indices, the very late-type stars indicate that there is a turnaround point in some of the scatter plots. However, as the sample is very sparse at very late spectral subtypes, these stars only have a minor impact on the correlation coefficients. 

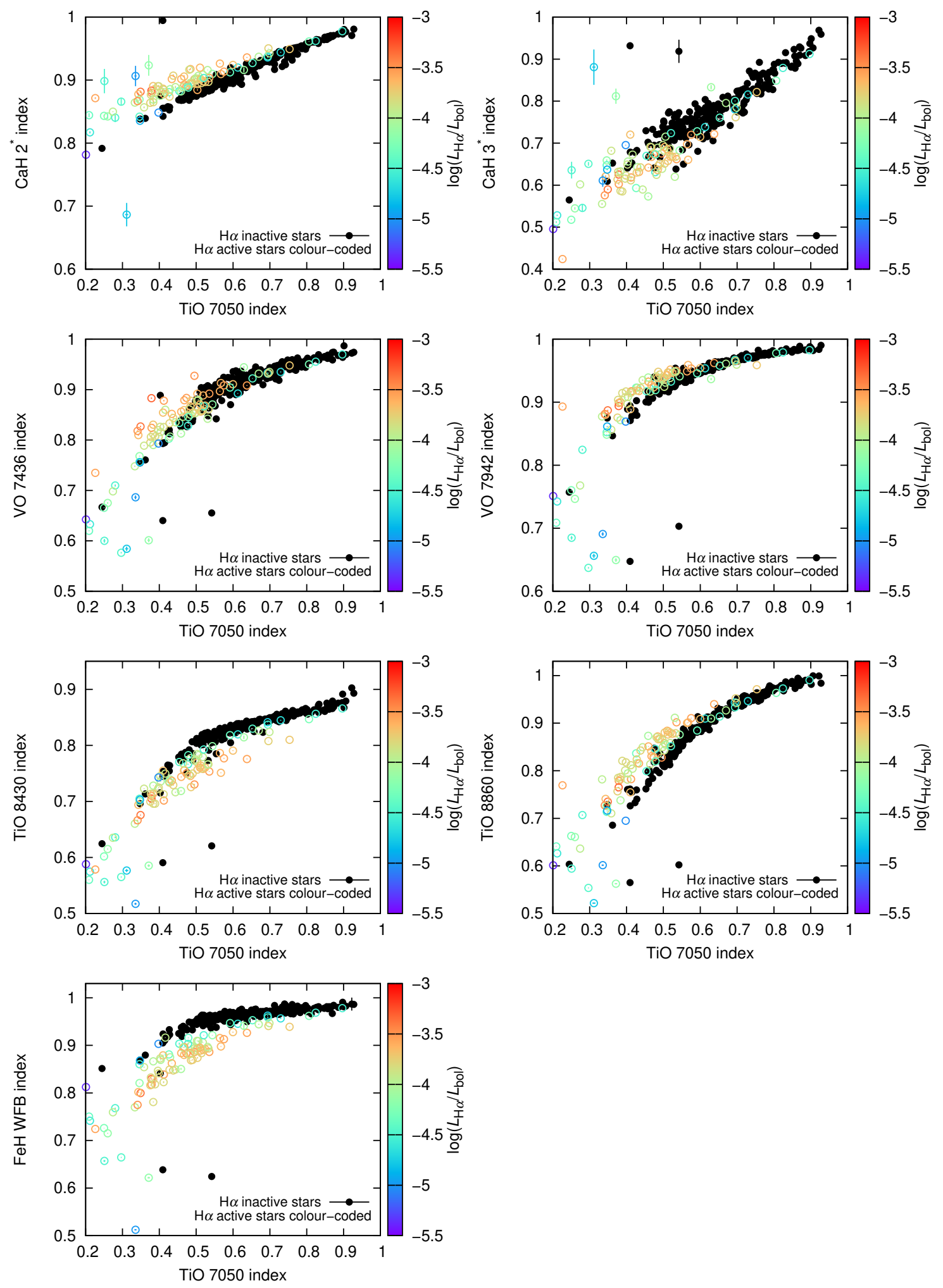

Figure 4.2.: Scatter plots of $\mathrm{TiO} 7050$ versus other photospheric absorption band indices. $\mathrm{H} \alpha$ inactive stars are shown in black, $\mathrm{H} \alpha$ active stars are colour-coded by normalised $\mathrm{H} \alpha$ luminosity. 

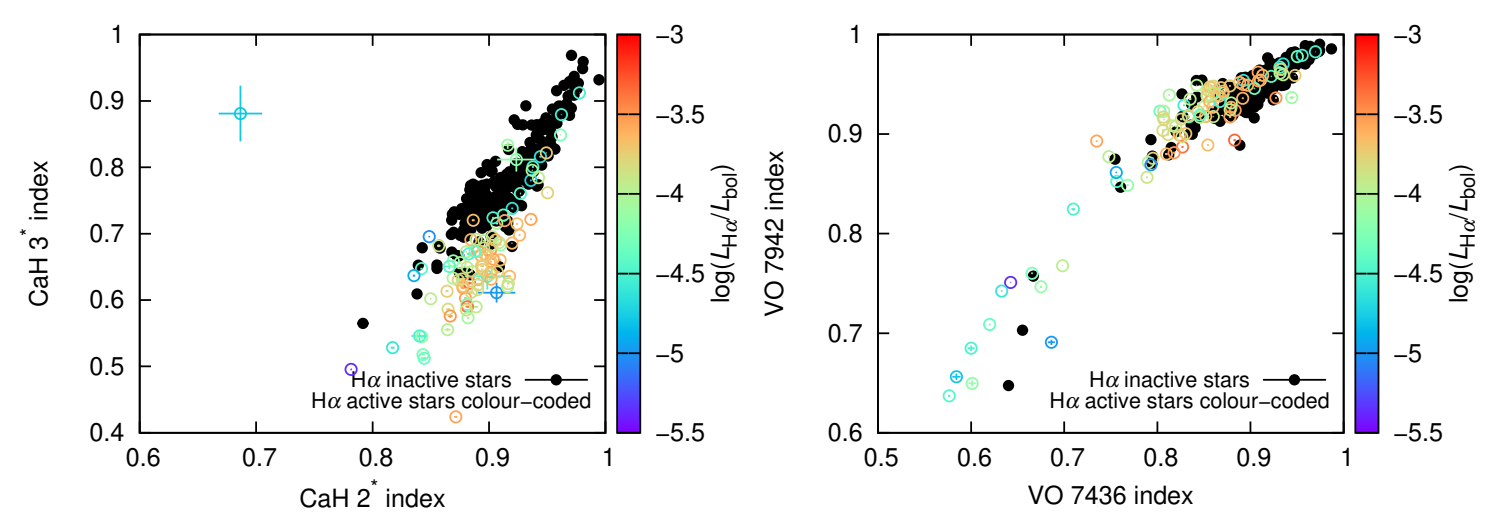

Figure 4.3.: Scatter plots of $\mathrm{CaH} 2^{*}$ versus $\mathrm{CaH} 3^{*}$ and of VO 7436 versus VO 7942. $\mathrm{H} \alpha$ inactive stars are shown in black, $\mathrm{H} \alpha$ active stars are colour-coded by normalised $\mathrm{H} \alpha$ luminosity.
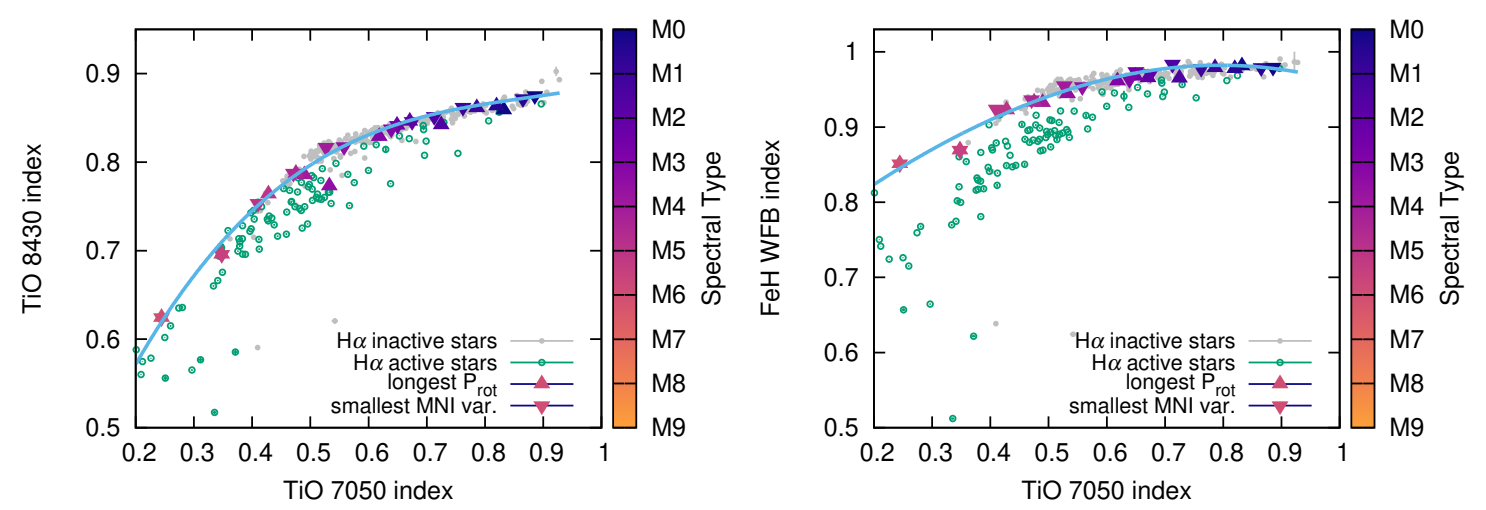

Figure 4.4.: Scatter plots of $\mathrm{TiO} 7050$ versus $\mathrm{TiO} 8430$ and of $\mathrm{TiO} 7050$ versus $\mathrm{FeH}$ WFB. $\mathrm{H} \alpha$ inactive stars are shown in grey, $\mathrm{H} \alpha$ active stars in green. Two sets of reference stars are shown as triagles colour-coded by spectral subtype. Blue curves are polynomial fits to the reference stars with the smallest MNI variations.

\subsubsection{Correlations with $\mathrm{H} \alpha$}

As described in the previous sections, the spectral-type dependences in Fig. 4.1 and the scatter plots in Figs. 4.2 and 4.3 revealed a difference between $\mathrm{H} \alpha$ inactive and $\mathrm{H} \alpha$ active stars. While the spread in the index values of the $\mathrm{H} \alpha$ inactive stars of each spectral subtype complicates the quantification of the activity effect as a function of spectral type, the scatter plots in general show a continuous, narrow line of $\mathrm{H} \alpha$ inactive stars. To avoid a selection bias from the definition of $\mathrm{H} \alpha$ active and $\mathrm{H} \alpha$ inactive, I define the inactivity line as a third-order polynomial fit to a set of reference stars classified as inactive by an independent measurement. The scatter plots of $\mathrm{TiO} 7050$ versus $\mathrm{TiO} 8430$ and of TiO 7050 versus FeH WFB are shown again in Fig. 4.4, but with the two sets of reference stars presented in Sect. 2.2.1 highlighted and colour-coded by their spectral subtype.

Because the M3.5 star with the longest known rotation period appears as an outlier in the scatter plot of $\mathrm{TiO} 7050$ versus $\mathrm{TiO} 8430$, I used the set of reference stars with the 

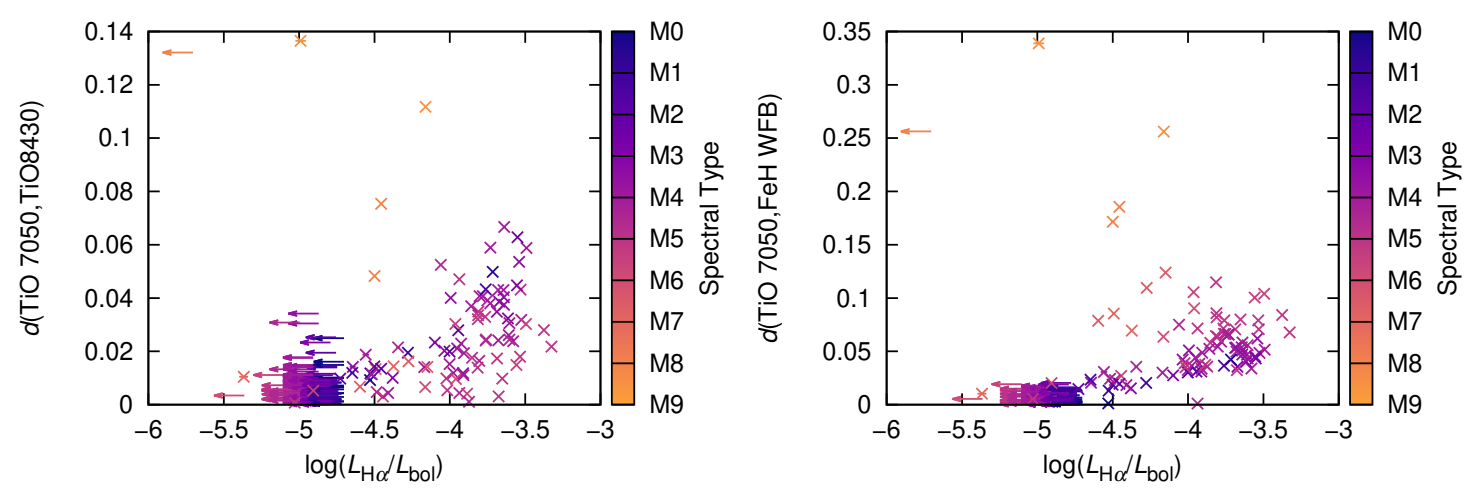

Figure 4.5.: $d(\mathrm{TiO} 7050, \mathrm{TiO} 8430)$ and $d(\mathrm{TiO} 7050, \mathrm{FeH}$ WFB $)$ as functions of normalised $\mathrm{H} \alpha$ luminosity. $\mathrm{H} \alpha$ inactive stars are shown as arrows starting at the normalised $\mathrm{H} \alpha$ luminosity corresponding to the activity threshold. Colours correspond to spectral subtypes.

smallest MNI variations to fit the inactivity line. The resulting polynomials are also shown in Fig. 4.4 and are given as follows:

$$
\begin{aligned}
\mathrm{TiO} 8430 & =g(\mathrm{TiO} 7050)=0.77 \cdot(\mathrm{TiO} 7050)^{3}-2.02 \cdot(\mathrm{TiO} 7050)^{2}+1.87 \cdot(\mathrm{TiO} 7050)+0.27 \\
\mathrm{FeH} \mathrm{WFB} & =h(\mathrm{TiO} 7050)=-0.09 \cdot(\mathrm{TiO} 7050)^{3}-0.29 \cdot(\mathrm{TiO} 7050)^{2}+0.63 \cdot(\mathrm{TiO} 7050)+0.71
\end{aligned}
$$

For each star, I then calculated the orthogonal distance $d(\mathrm{TiO} 7050, \mathrm{TiO} 8430)$ from its position in the ( $\mathrm{TiO} 7050$, TiO 8430)-plane to the polynomial $g$, and the orthogonal distance $d$ (TiO 7050, FeH WFB) from its position in the (TiO 7050, FeH WFB)-plane to the polynomial $h$. The resulting distances are shown as a function of the normalised $\mathrm{H} \alpha$ luminosity in Fig. 4.5.

In both the ( $\mathrm{TiO} 7050, \mathrm{TiO} 8430$ )- and the ( $\mathrm{TiO} 7050, \mathrm{FeH} \mathrm{WFB}$ )-plane, larger distance values are more common for stars with higher normalised $\mathrm{H} \alpha$ luminosities, and the very late-type stars are outliers because the polynomials can not fit the turnaround point that occurs at very late spectral subtypes. While the $d$ (TiO 7050, FeH WFB) values for most $\mathrm{H} \alpha$ active stars are larger than for $\mathrm{H} \alpha$ inactive stars, this is not true for $d$ (TiO 7050, TiO 8430), as the polynomial $g$ is close to the lower end of the line of $\mathrm{H} \alpha$ inactive stars in the mid-type range.

\subsection{Temporal variability}

In the same way as for the chromospheric indicators in Sect. 3.3, I calculated the absolute variations of the photospheric band indices from the individual spectra of each star and divided them by the index value derived from the co-added template spectrum to obtain the relative variation. The relative variations are shown as a function of the index value derived from the co-added template spectrum in Fig. 4.6. 

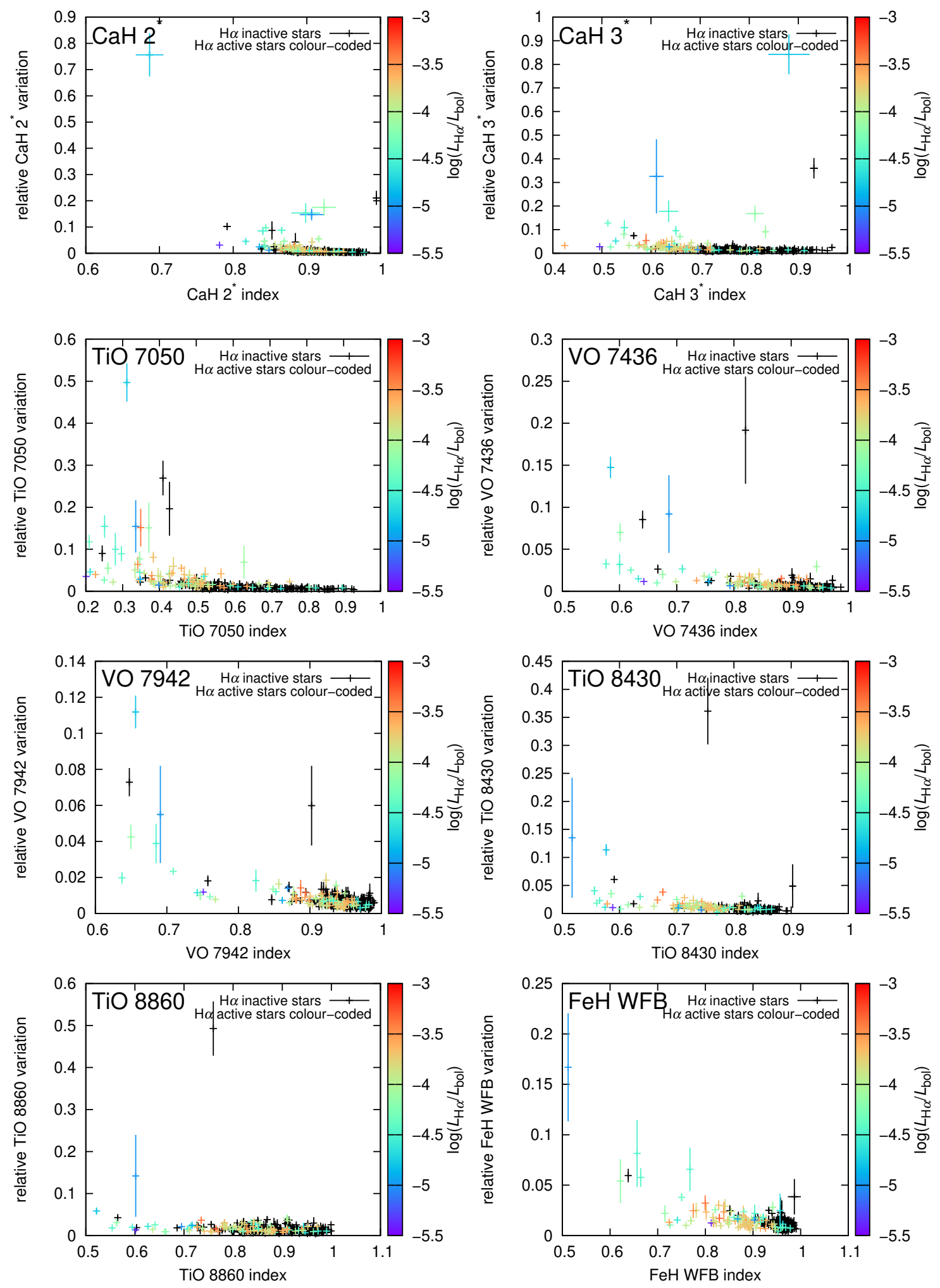

Figure 4.6.: Relative index variations as functions of index values. $\mathrm{H} \alpha$ inactive stars are shown in black, $\mathrm{H} \alpha$ active stars are colour-coded by normalised $\mathrm{H} \alpha$ luminosity. 

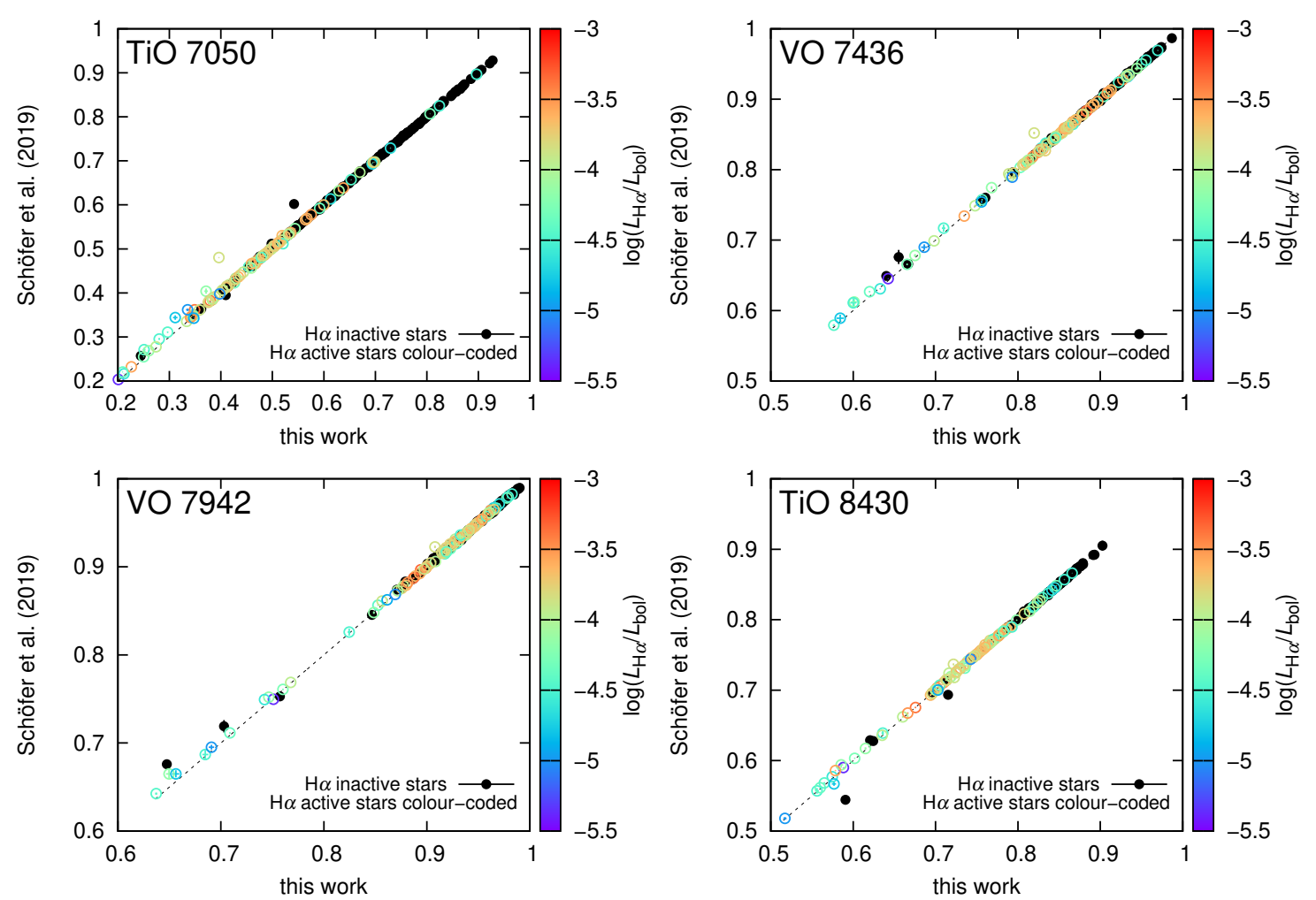

Figure 4.7.: Comparison of photospheric absorption band indices derived in this thesis and in Schöfer et al. (2019). H $\alpha$ inactive stars are shown in black, $\mathrm{H} \alpha$ active stars are colour-coded by normalised $\mathrm{H} \alpha$ luminosity. Dashed black lines mark the one-to-one relation.

The relative variations generally decrease with increasing index value for all indices, outliers have large error bars. As the SNR is lower for stars with later spectral types and thus lower index values, the larger relative variations are likely caused by larger statistical fluctuations. There also appear to be larger relative variations for $\mathrm{H} \alpha$ active stars, but this is likely a selection effect, as the fraction of $\mathrm{H} \alpha$ active stars is higher at later spectral subtype and lower index values.

\subsection{Discussion}

For the four photospheric absorption band indices that were already presented in Schöfer et al. (2019), I compare the index values presented in this thesis with the earlier results in Fig. 4.7. The two significant outliers in TiO 7050 and VO 7436 are J10584-107 and J08536-034. While the former star also appeared as an outlier in the comparison of the chromospheric indicators, the latter star is the only M9.0 star in the sample and has a low SNR in this wavelength range. In VO 7942, two additional very late-type stars appear as outliers with J19255+096 and J04198+425, whereas in TiO 8430, J19255+096, and the two M5.0 stars J06318+414 and J10584-107 are the strongest outliers. 
The $\mathrm{CaH} 2^{*}$, $\mathrm{CaH} 3^{*}$, and $\mathrm{TiO} 7050$ indices measure the strengths of the same absorption band heads as the $\mathrm{CaH} 2, \mathrm{CaH} 3$, and TiO 2 indices defined by Reid et al. (1995). In Fig. 4.8, I compare my indices to the index values from low-resolution spectra reported by the Palomar/Michigan State University Survey (PMSU, Reid et al. 1995; Hawley et al. 1996) and by Alonso-Floriano et al. (2015, AF15). The CaH 2 index was only measured by the PMSU and uses the denominator range from $7042 \AA$ to $7046 \AA$. This is at far longer wavelengths than the numerator range, whereas the denominator range of the $\mathrm{CaH} 2 *$ index is close to the numerator range. Therefore, the $\mathrm{CaH} 2$ index values are distinctly lower than the $\mathrm{CaH} \mathrm{2*}$ index values. $\mathrm{H} \alpha$ active stars generally have lower $\mathrm{CaH} 2$ index values than $\mathrm{H} \alpha$ inactive stars with the same $\mathrm{CaH} 2^{*}$ index value. The $\mathrm{CaH} 3$ index uses a significantly larger numerator range than the $\mathrm{CaH} 3^{*}$, while the denominator ranges are similar. This results in similar index values. In general, the $\mathrm{CaH} 3$ index values reported by PMSU are below and the $\mathrm{CaH} 3$ index values reported by AF15 are above the one-toone relation. The overlap between my sample and the PMSU and AF15 samples include mainly $\mathrm{H} \alpha$ inactive earlier-type and $\mathrm{H} \alpha$ active later-type stars, therefore, no distinct trends with increasing $\mathrm{H} \alpha$ activity can be seen. The strong outlier in the comparison with the $\mathrm{CaH} 3$ index values from AF15 is the active M3.5 star J02002+130 that also appears as a strong outlier in the comparison of the $\mathrm{TiO} 7050$ and $\mathrm{TiO} 2$ index values. The $\mathrm{TiO} 2$ index uses slightly narrower numerator and denominator ranges than the $\mathrm{TiO} 7050$ index. For both PMSU and AF15, the TiO 2 index values are above the one-to-one relation with a constant offset.

In Fig. 4.9, I show again the scatter plots of $\mathrm{TiO} 7050$ versus $\mathrm{TiO} 8430$ and $\mathrm{FeH}$ WFB, but colour-coded by the effective temperatures $T_{\text {eff }}$ and metallicities $[\mathrm{Fe} / \mathrm{H}]$ derived by Schweitzer et al. (2019). The inactivity line polynomials defined in Sect. 4.2.2 are also shown again. As expected from theory and from the spectral-type dependence of the indices, $T_{\text {eff }}$ decreases with decreasing indices until the turnaround point that is caused by the formation of dust that changes the contrast between the numerator and denominator ranges in very late $\mathrm{M}$ dwarfs (e.g. Tsuji et al. 1996; Allard et al. 2001). However, the metallicities show an unexpected behaviour, as the metal-rich stars appear below the inactivity line only for $\mathrm{TiO} 7050>0.7$, whereas at lower index values, the stars below the inactivity line appear to be generally metal-poor. As most stars with large distances to the inactivity line are $\mathrm{H} \alpha$ active, and $\mathrm{H} \alpha$ active stars tend to be younger than $\mathrm{H} \alpha$ inactive stars as shown in the previous section, higher metallicities are expected. The inactivity line and the orthogonal distances from it as defined in Sect. 4.2.2 may therefore be helpful to better constrain metallicities. There is, however, room to improve the inactivity line fit. As the $\mathrm{H} \alpha$ active stars are located below the line of $\mathrm{H} \alpha$ inactive stars, it is plausible that the most inactive stars are located at the upper boundary of this line. Therefore, an upper envelope fit to this line might yield improved results. To improve the results for very late-type stars, the inactivity line would also need to follow the turnaround point. However, this is hard to fit, as the sample of very late-type stars is sparse and does not contain $\mathrm{H} \alpha$ inactive stars.

Orthogonal distances from an improved inactivity line may also help to understand the activity effects on the photospheric bands. Both PMSU and AF15 reported for their TiO 2 index a similar behaviour with activity as I described for the TiO 7050 index. Activity 

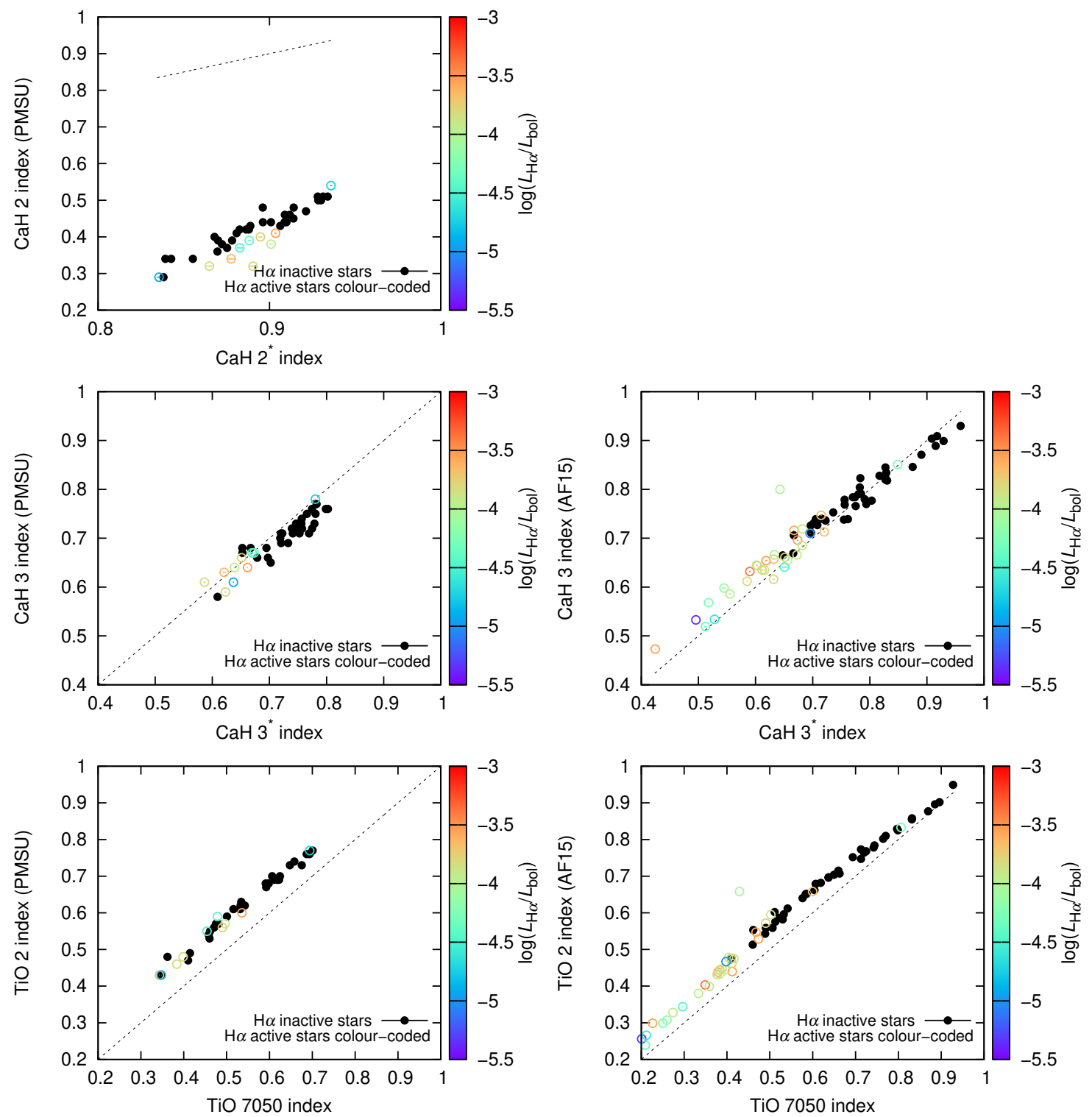

Figure 4.8.: Comparison of photospheric absorption band indices with indices from PMSU and AF15. $\mathrm{H} \alpha$ inactive stars are shown in black, $\mathrm{H} \alpha$ active stars are colour-coded by normalised $\mathrm{H} \alpha$ luminosity. Dashed black lines mark the one-to-one relation. The $\mathrm{CaH} \mathrm{2*}$ index is consistently higher than the $\mathrm{CaH} 2$ index because of the different denominator range. 

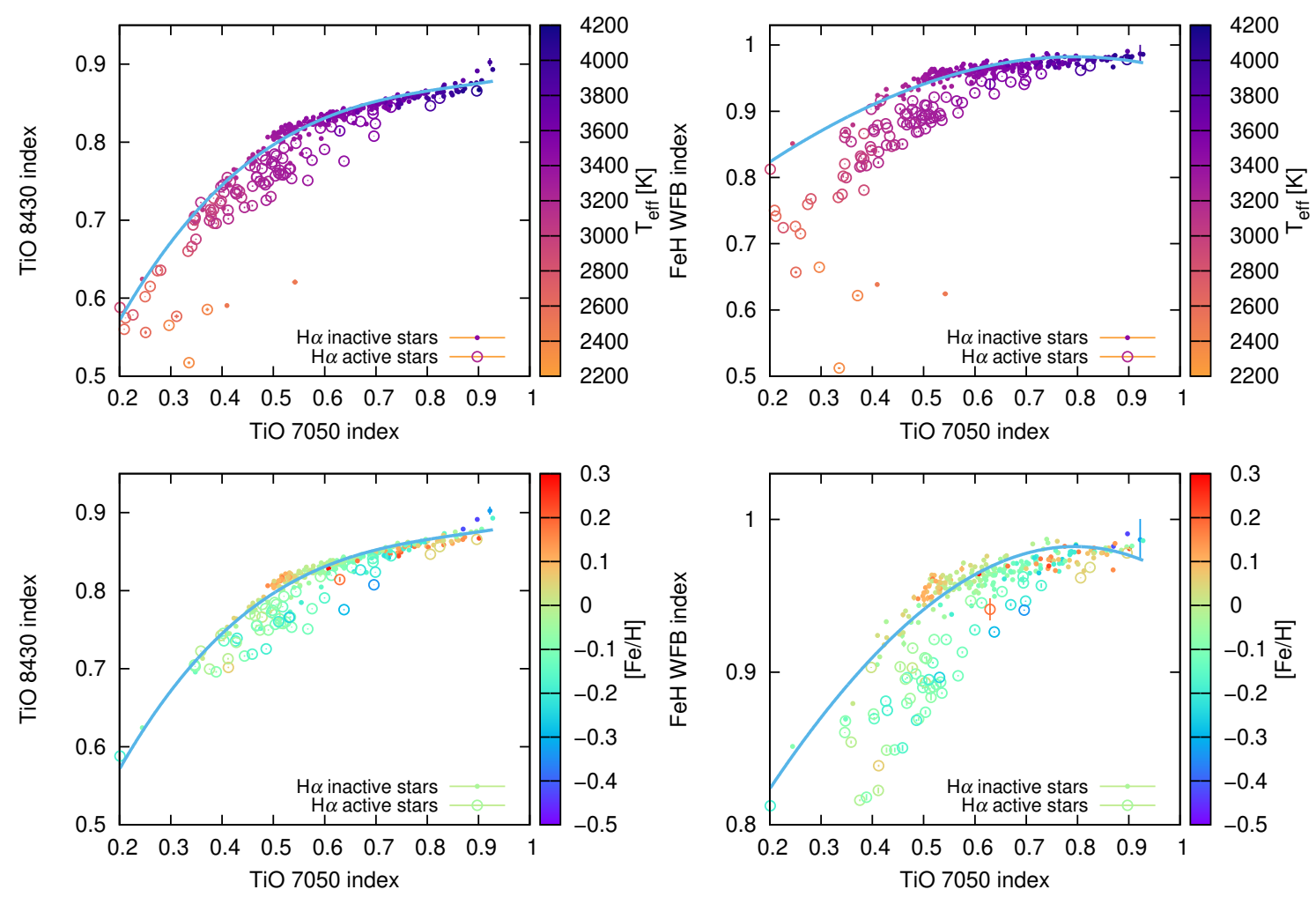

Figure 4.9.: Scatter plots of $\mathrm{TiO} 7050$ versus $\mathrm{TiO} 8430$ and of $\mathrm{TiO} 7050$ versus $\mathrm{FeH}$ WFB, colourcoded by effective temperature and metallicity. Blue curves are the same polynomial fits to the reference stars with the smallest MNI variations as in Fig. 4.4

appears to affect different photospheric bands in different ways, so this did not affect the spectral types that were predominantly derived by PMSU and AF15 using a combination of multiple photospheric band indices. While the temperature dependence of TiO bands has also been used to measure starspot areas and temperatures (e.g. Neff et al. 1995; O'Neal et al. 1998), starspots should affect all photospheric bands. As shown in Schöfer et al. (2019), stellar rotation and magnetic fields may explain the observations.

The absolute or relative variations of the photospheric absorption band indices appear to be dominated by statistical fluctuations. However, the temporal variability of the indices still contains information about the stellar surface for some stars, as demonstrated in the next chapter. 



\section{Rotational variation of activity indicators}

As described in Sect. 1.4.1, the rotation of a star can lead to periodic modulations of its light curve because over the course of one rotation, active regions appear and disappear on the hemisphere faced towards the observer. Similarly, the impact of active regions on the disc-integrated spectrum changes, leading to modulations of spectroscopic activity indicators.

This chapter further analyses the temporal variability of the activity indicators presented in Chapters 3 and 4 with a focus on periodicities. While Sect. 5.1 again extends the analysis presented in Schöfer et al. (2019) to include additional photospheric band indices and observations after July 2018, Sect. 5.2 summarises a more detailed analysis of four individual stars (Schöfer et al. submitted). I discuss the results presented in this chapter in Sect. 5.3.

\subsection{Sample overview}

Before searching for periodic changes in the activity indicators of each star, I excluded data points outlying by more than two standard deviations ( $2 \sigma$ clipping). This removes poor spectra and strong flaring events that are not expected to occur periodically from the time series. In the cases of $\mathrm{He} 10833$ and $\mathrm{Pa} \beta$, measurements from spectra with telluric contamination were also excluded as described in Sect. 2.2. For each star and each indicator, I then calculated the generalised Lomb-Scargle (GLS) periodogram (Zechmeister \& Kürster 2009) and identified the frequency with the highest GLS power as the bestfit frequency. After subtracting the best-fit frequency sine curve from each time series, I again calculated the GLS periodograms to obtain the second-best-fit frequencies and, after another iteration, the third-best-frequencies. The frequency space is restricted to frequencies between 0 and $1 \mathrm{~d}^{-1}$ because most stars were observed at most once per night, leading to a poor sampling of higher frequencies.

I now compare the obtained best-fit frequencies of the activity indicators with the rotation frequencies $1 / P_{\text {rot }}$ of the stars with a known rotation period $P_{\text {rot }}$ as listed in Table A.1. Of these 169 stars, 21 have a rotation period shorter than $1 \mathrm{~d}$ and are therefore excluded from this comparison. The histogram in Fig. 5.1 shows the fraction of the 148 stars with 


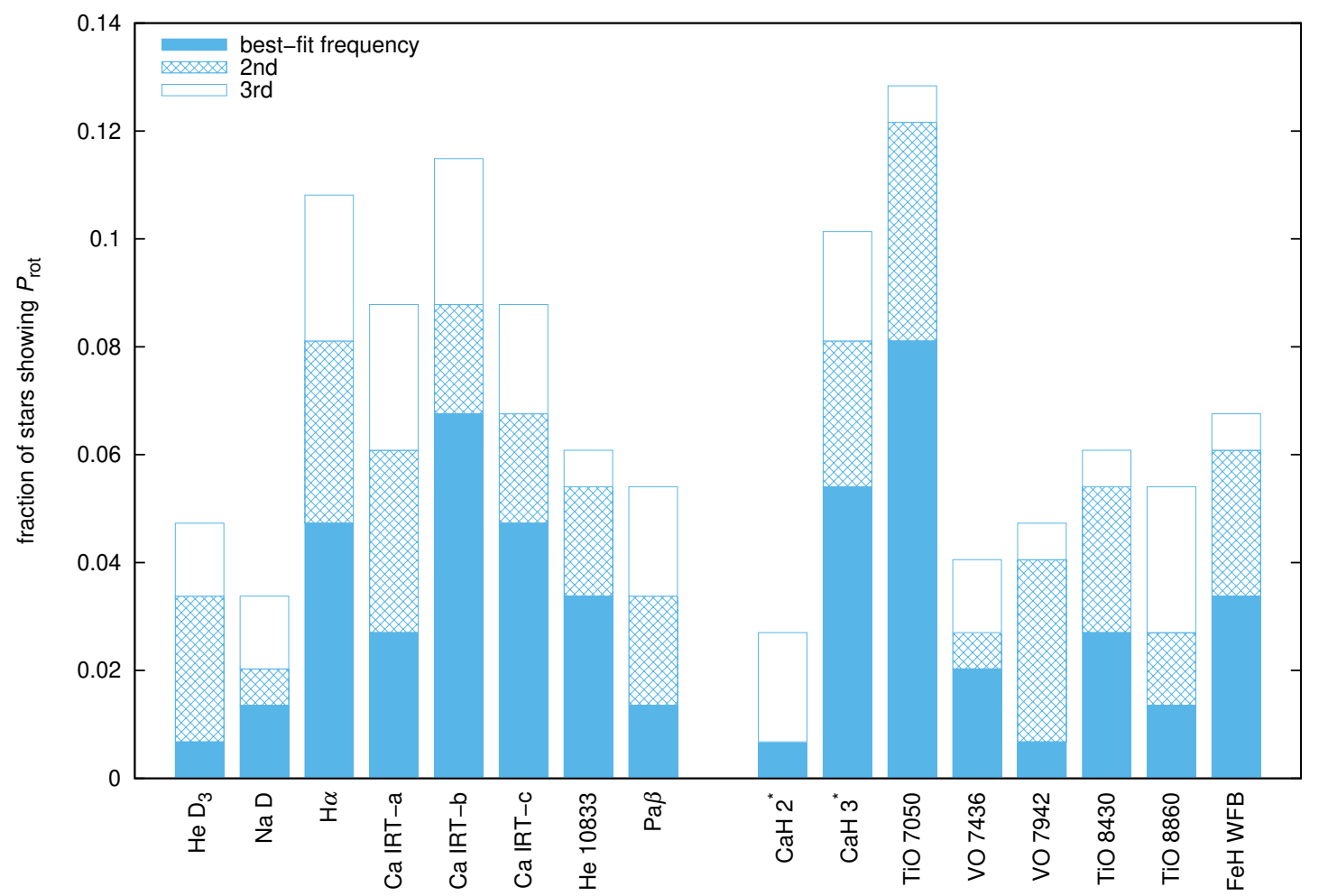

Figure 5.1.: Fraction of stars showing the rotation frequency as one of the three best-fit frequencies in each activity indicator

$P_{\text {rot }}>1 \mathrm{~d}$ for that the best-fit frequencies for each indicator are within three times the error bar of the rotation frequency.

With 19 stars showing the rotation frequency as one of the three best-fit frequencies, $\mathrm{TiO} 7050$ is the indicator that most commonly varies with the rotation period. Ca IRT-b, $\mathrm{H} \alpha$, and $\mathrm{CaH} 3^{*}$ are the only other indicators that show the rotation frequency as one of the three best-fit frequencies for more than $10 \%$ of the stars with $P_{\text {rot }}>1 \mathrm{~d}$. For every indicator, the rotation frequency appears among the three best-fit frequencies at least in four stars and as the single best-fit frequency in at least one star.

Overall, 69 stars show the rotation frequency as one of the three best-fit frequencies in at least one indicator. For only 18 of these stars, is this true in more than two indicators. The stricter criterion that the rotation frequency appears as the single best-fit frequency in more than two indicators is satisfied by only seven stars, of which I analyse four in detail in the next section. 
Table 5.1.: Basic parameters and number of CARMENES observations of the four stars discussed in Sect. 5.2. References for the spectral types, $P_{\text {rot }}$, and $v \sin i$ are given in Table A.1.

\begin{tabular}{llccccr}
\hline Karmn & Name & SpT & $\begin{array}{c}P_{\text {rot }} \\
{[\mathrm{d}]}\end{array}$ & $\begin{array}{c}v \sin i \\
{\left[\mathrm{~km} \mathrm{~s}^{-1}\right]}\end{array}$ & $\log \left(L_{\mathrm{H} \alpha} / L_{\text {bol }}\right)$ & $N_{\text {obs }}$ \\
\hline J01025+716 & Ross 318 & M3.0 & $51.5 \pm 2.6$ & $<2$ & $\ldots$ & 120 \\
J07446+035 & YZ CMi & M4.5 & $2.78 \pm 0.01$ & $4.0 \pm 1.5$ & $-3.60 \pm 0.01$ & 51 \\
J18174+483 & TYC 3529-1437-1 & M2.0 & $15.8 \pm 0.1$ & $3.1 \pm 1.0$ & $-4.00 \pm 0.01$ & 71 \\
J22468+443 & EV Lac & M3.5 & $4.38 \pm 0.03$ & $5.9 \pm 0.1$ & $-3.64 \pm 0.01$ & 107 \\
\hline
\end{tabular}

\subsection{Four individual stars}

For a more detailed analysis of the periodic modulations of the activity indicators, I selected four stars that not only showed the rotation frequency as the best-fit frequency in more than two indicators, but also were observed more than 40 times before January 2019. This additional requirement allows for a study of the stability of the signals seen in the GLS periodograms. The four stars and their basic parameters are listed in Table 5.1. While Ross 318 is an $\mathrm{H} \alpha$ inactive slow rotator, TYC 3529-1437-1 shows moderate $\mathrm{H} \alpha$ emission, but still rotates relatively slowly. In contrast, YZ CMi and EV Lac are very active fast rotators.

I calculated the GLS periodograms for these four stars not only of the activity indicators presented in this thesis, but also of the differential line width (dLW), the chromatic index (CRX), the radial velocity (RV), and the full-width at half-maximum (FWHM), contrast, and bisector inverse slope (BIS, Queloz et al. 2001) of the cross-correlation function (CCF) of the spectrum with a weighted binary mask. dLW and CRX were defined by Zechmeister et al. (2018) and calculated together with the RV by Dr. Mathias Zechmeister using SERVAL and a correction for an instrumental nightly zero-point RVoffset (Trifonov et al. 2018; Tal-Or et al. 2019). The CCF parameters were calculated by Marina Lafarga (Institut de Ciències de l'Espai, Barcelona, Spain) as described in Lafarga et al. (2020). All these additional indicators were calculated separately for the VIS and the NIR channel.

The GLS periodograms and the window functions for both spectrograph channels are shown in Fig. B.1 for Ross 318, Fig. B.2 for YZ CMi, Fig. B.3 for TYC 3529-1437-1, and Fig. B.4 for EV Lac. To quantify the significance of a signal at frequency $f$ in the GLS periodogram, the probability $p(f)$ that a power higher than the GLS power at $f$ could be caused purely by Gaussian noise can be calculated. Table 5.2 tabulates the logarithmic probabilities $p\left(1 / P_{\text {rot }}\right)$ for the highest GLS power in the $3 \sigma$ confidence interval around $1 / P_{\text {rot }}$ for all indicators of the four stars.

Ross 318 shows a significant signal at $1 / P_{\text {rot }}$ in all chromospheric indicators with the exceptions of $\mathrm{He}_{3}$ and $\mathrm{Na} \mathrm{D}$, in the $\mathrm{CaH} 3^{*}$, TiO 7050, VO 7436, and $\mathrm{TiO} 8860$ indices, and in the additional indicators with the exceptions of the CCF contrast in both channels 
Table 5.2.: $\log p\left(1 / P_{\text {rot }}\right)$ for all indicators of Ross 318, YZ CMi, TYC 3529-1437-1, and EV Lac. Logarithmic probabilities more negative than -2.87 indicate a significance higher than $3 \sigma$ and are highlighted in boldface.

\begin{tabular}{|c|c|c|c|c|}
\hline Indicator & Ross 318 & YZ CMi & TYC 3529-1437-1 & EV Lac \\
\hline $\mathrm{He} \mathrm{D}_{3}$ & -1.68 & -2.28 & -2.01 & -1.51 \\
\hline $\mathrm{Na} \mathrm{D}$ & -2.21 & -1.10 & -3.51 & -2.68 \\
\hline $\mathrm{H} \alpha$ & -7.14 & -2.29 & -5.12 & -4.84 \\
\hline Ca IRT-a & -3.05 & -1.59 & -3.34 & -3.29 \\
\hline Ca IRT-b & -6.52 & -1.63 & -4.54 & -3.02 \\
\hline Ca IRT-c & -6.87 & -1.33 & -4.33 & -2.77 \\
\hline He 10833 & -5.91 & -1.45 & -0.98 & -3.43 \\
\hline $\mathrm{Pa} \beta$ & -3.15 & -1.39 & -0.75 & -0.59 \\
\hline $\mathrm{CaH} 2^{*}$ & -0.41 & -1.72 & -1.19 & -0.88 \\
\hline $\mathrm{CaH} 3^{*}$ & -5.88 & -9.04 & -1.17 & -4.94 \\
\hline TiO 7050 & -11.91 & -19.86 & -2.68 & -11.04 \\
\hline VO 7436 & -3.01 & -7.44 & -1.42 & -2.47 \\
\hline VO 7942 & -1.13 & -1.50 & -1.71 & -1.72 \\
\hline TiO 8430 & -1.60 & -13.35 & -1.49 & -5.10 \\
\hline TiO 8860 & $-\mathbf{5 . 3 7}$ & -2.66 & -0.97 & -0.56 \\
\hline FeH WFB & -1.01 & -6.55 & -1.15 & -2.83 \\
\hline dLW VIS & -6.11 & -8.49 & -1.83 & -11.90 \\
\hline dLW NIR & -7.63 & -2.69 & -2.24 & -9.11 \\
\hline CRX VIS & -3.38 & -14.60 & -2.02 & -2.59 \\
\hline CRX NIR & -5.04 & -11.57 & -2.06 & -1.44 \\
\hline RV VIS & -7.82 & -18.72 & -4.70 & -4.61 \\
\hline RV NIR & $-\mathbf{3 . 3 9}$ & -14.03 & -3.36 & -2.70 \\
\hline CCF FWHM VIS & -5.38 & -11.77 & -1.35 & -4.86 \\
\hline CCF FWHM NIR & -15.18 & -9.18 & -2.19 & -4.16 \\
\hline CCF contrast VIS & -2.57 & -12.22 & -1.77 & -7.54 \\
\hline CCF contrast NIR & -1.62 & -3.77 & -1.60 & -6.36 \\
\hline CCF BIS VIS & -1.46 & -19.23 & -1.11 & -3.51 \\
\hline CCF BIS NIR & -6.69 & -6.32 & -1.62 & -2.27 \\
\hline
\end{tabular}


and CCF BIS VIS. However, in Ca IRT-a, $\mathrm{Pa} \beta, \mathrm{CaH} 3^{*}$, VO 7436, TiO 8860, the dLW in both channels, and RV NIR, the periodogram peak at $1 / P_{\text {rot }}$ is not the highest. In contrast, YZ CMi shows a significant signal at $1 / P_{\text {rot }}$ in none of the chromospheric indicators, but in all other indicators with the exceptions of $\mathrm{CaH} 2^{*}, \mathrm{VO} 7942$, TiO 8860, and dLW NIR. The signals are generally more significant than the signals seen for Ross 318 , and correspond to the highest periodogram peaks in all cases but CCF contrast NIR.

In the case of TYC 3529-1437-1, only $\mathrm{Na}$ D, $\mathrm{H} \alpha$, the Ca IRT lines, and the RV in both channels show a significant signal in $1 / P_{\text {rot }}$. While these signals are generally weaker than the signals seen for $\mathrm{YZ} \mathrm{CMi,} \mathrm{they} \mathrm{correspond} \mathrm{to} \mathrm{the} \mathrm{highest} \mathrm{periodogram} \mathrm{peaks} \mathrm{in}$ all those indicators but RV VIS, where a higher peak appears at the first harmonic of the rotation frequency, $2 / P_{\text {rot }}$. Finally, EV Lac shows a significant signal at $1 / P_{\text {rot }}$ in $\mathrm{H} \alpha$, Ca IRT-a, Ca IRT-b, He 10833, CaH 3*, TiO 7050, TiO 8430, the dLW in both channels, RV VIS, and all CCF parameters but CCF BIS NIR. While the highest periodogram peak is also located at $1 / P_{\text {rot }}$ in $\mathrm{H} \alpha, \mathrm{CaH} 3^{*}, \mathrm{TiO} 7050$, TiO 8430 , the dLW in both channels, and CCF contrast VIS, the highest periodogram peak in RV VIS, CCF FWHM VIS, and CCF BIS VIS appears at $2 / P_{\text {rot }}$. The CRX in both channels and RV NIR also show a significant highest peak at $2 / P_{\text {rot }}$ despite not showing a significant signal at $1 / P_{\text {rot }}$. For the Ca IRT lines, the highest peak appears at $f=1 / P_{\text {rot }}+0.03 \mathrm{~d}^{-1}$. The window function suggests that this is a yearly alias of the rotation frequency.

For $\mathrm{H} \alpha$ and $\mathrm{TiO} 7050$, which are the indicators showing the strongest signals among the chromospheric and photospheric indicators, respectively, and for RV VIS, which is the only indicator that shows a significant signal at $1 / P_{\text {rot }}$ for all four stars, I tested the stability of the signals over time. To do this, I calculated separate GLS periodograms for each possible subset of 21 consecutive data points. This is a compromise between creating a relevant amount of subsets and ensuring sufficient sampling in each subset. A graphical representation of the results are the rolling periodograms explained in Appendix B and shown in Fig. B.5 for Ross 318, in Fig. B.6 for YZ CMi, in Fig. B.7 for TYC 35291437-1, and in Fig. B.8 for EV Lac. To quantify the significance of the signals in the rolling periodograms, I calculated $\log p\left(1 / P_{\text {rot }}\right)$ and $\log p\left(2 / P_{\text {rot }}\right)$ for each subset. These logarithmic probabilities are shown as a function of the median Barycentric Julian Date (BJD) of the data subset in Fig. 5.2 for Ross 318, in Fig. 5.3 for YZ CMi, in Fig. 5.4 for TYC 3529-1437-1, and in Fig. 5.5 for EV Lac.

In the rolling periodograms for Ross 318 , significant signals at $1 / P_{\text {rot }}$ are seen in RV VIS in the first half of the full time baseline, and in $\mathrm{H} \alpha$ and $\mathrm{TiO} 7050$ in the second half. Barely significant signals at $2 / P_{\text {rot }}$ appear occasionally, but are generally weaker than signals at $1 / P_{\text {rot }}$ in the same data subsets of the same indicator. However, the long rotation period of $51.5 \pm 2.6 \mathrm{~d}$ is not well sampled in most subsets, leading to weak signals in general. The rolling periodograms for YZ CMi reveal very stable signals at $1 / P_{\text {rot }}$ in $\mathrm{TiO} 7050$ and RV VIS, and no significant signals at $1 / P_{\text {rot }}$ in $\mathrm{H} \alpha$ or at $2 / P_{\text {rot }}$ in any indicator. While the signal at the rotation frequency more significant in $\mathrm{TiO} 7050$ in the first data subsets, it is more significant in RV VIS in the last data subsets. In addition, an insignificant signal at $1 / P_{\text {rot }}$ in $\mathrm{H} \alpha$ approaches the $3 \sigma$ significance threshold in the last data subsets. 


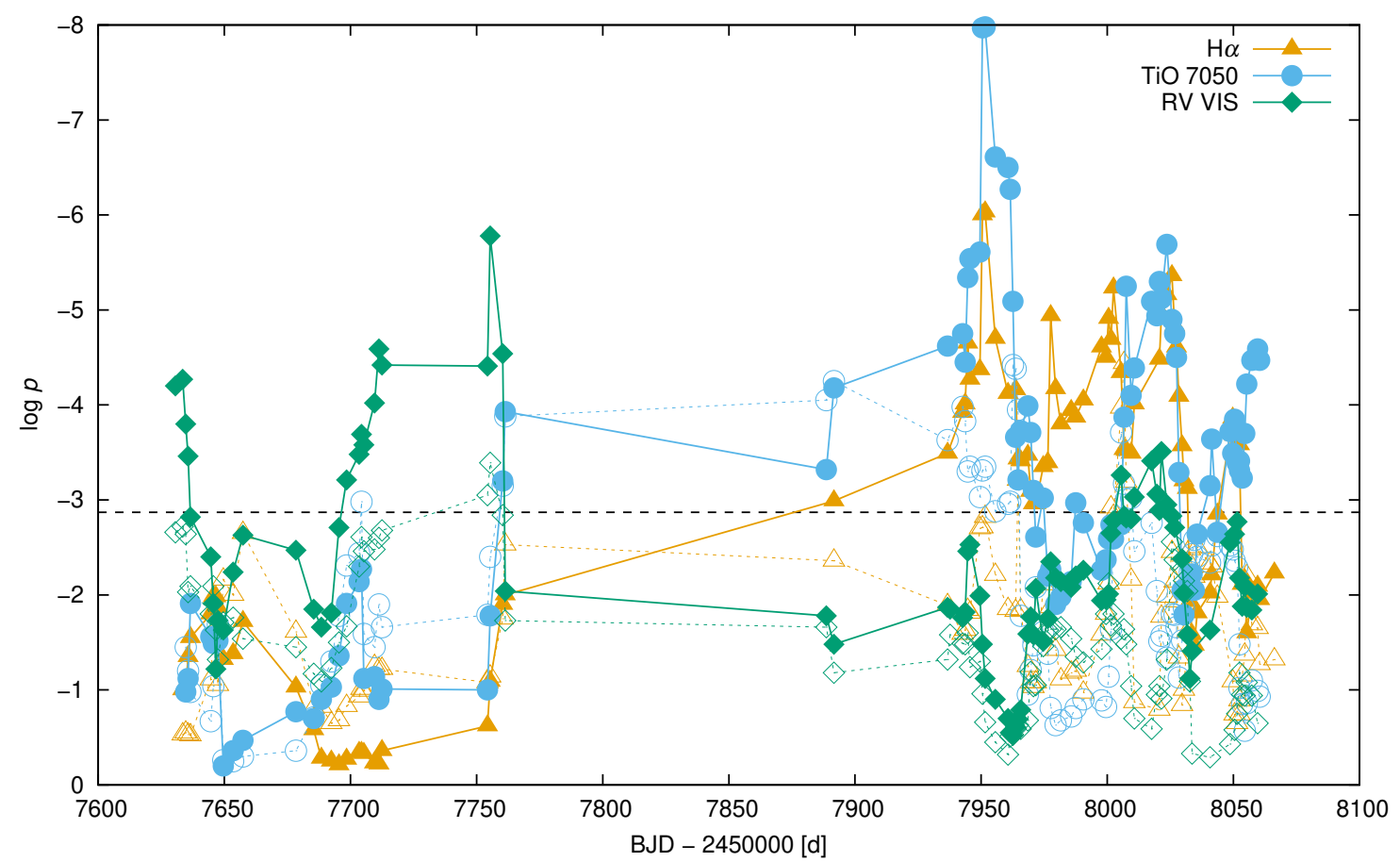

Figure 5.2.: $\log p\left(1 / P_{\text {rot }}\right)$ (filled symbols connected with solid lines) and $\log p\left(2 / P_{\text {rot }}\right)$ (empty symbols connected with dashed lines) from GLS periodograms of each subset of $\mathrm{H} \alpha$ (orange triangles), TiO 7050 (blue circles), and RV VIS (green diamonds) data points for Ross 318. The dashed black line marks the $3 \sigma$ significance level.

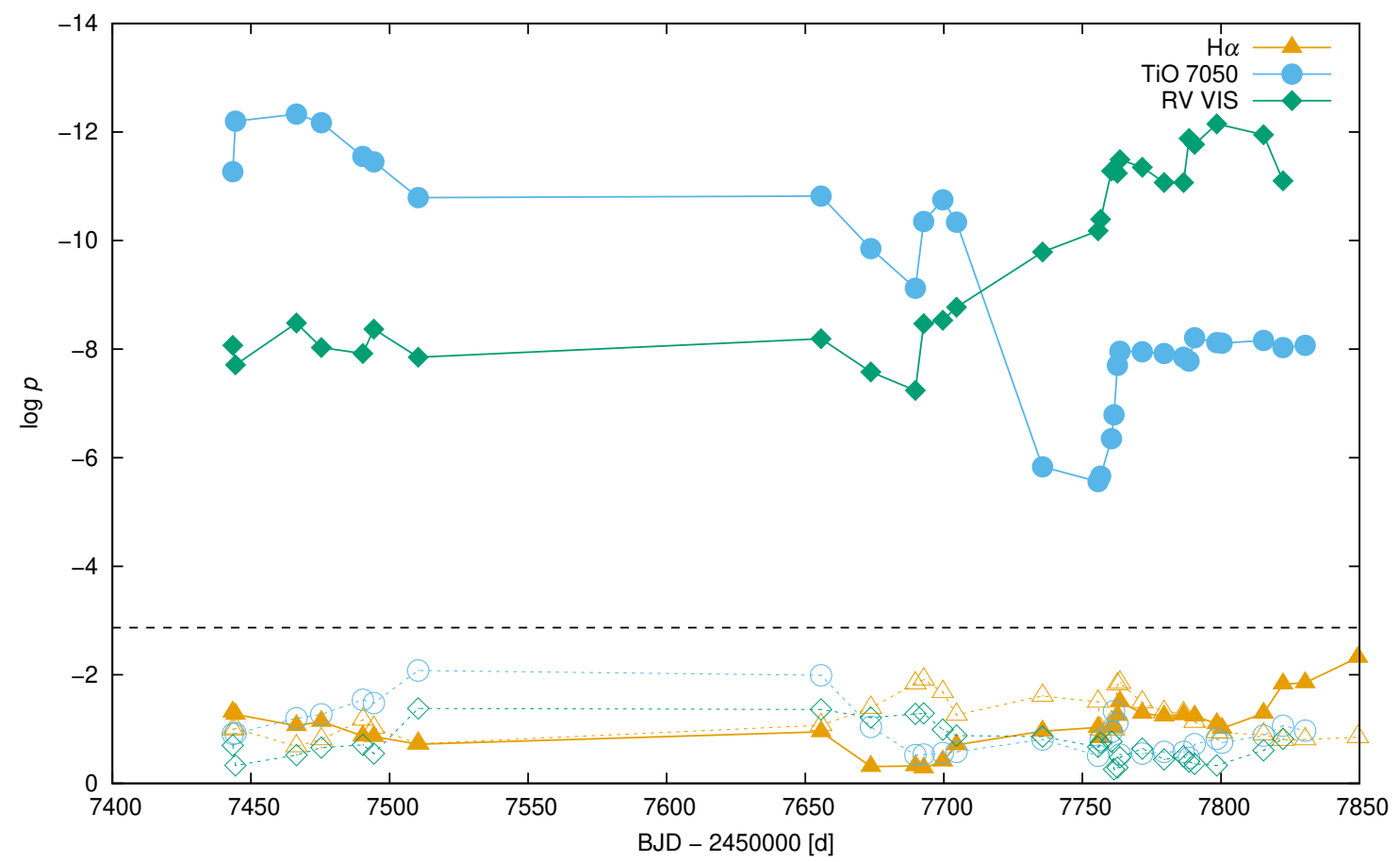

Figure 5.3.: Same as Fig. 5.2, but for YZ CMi. 


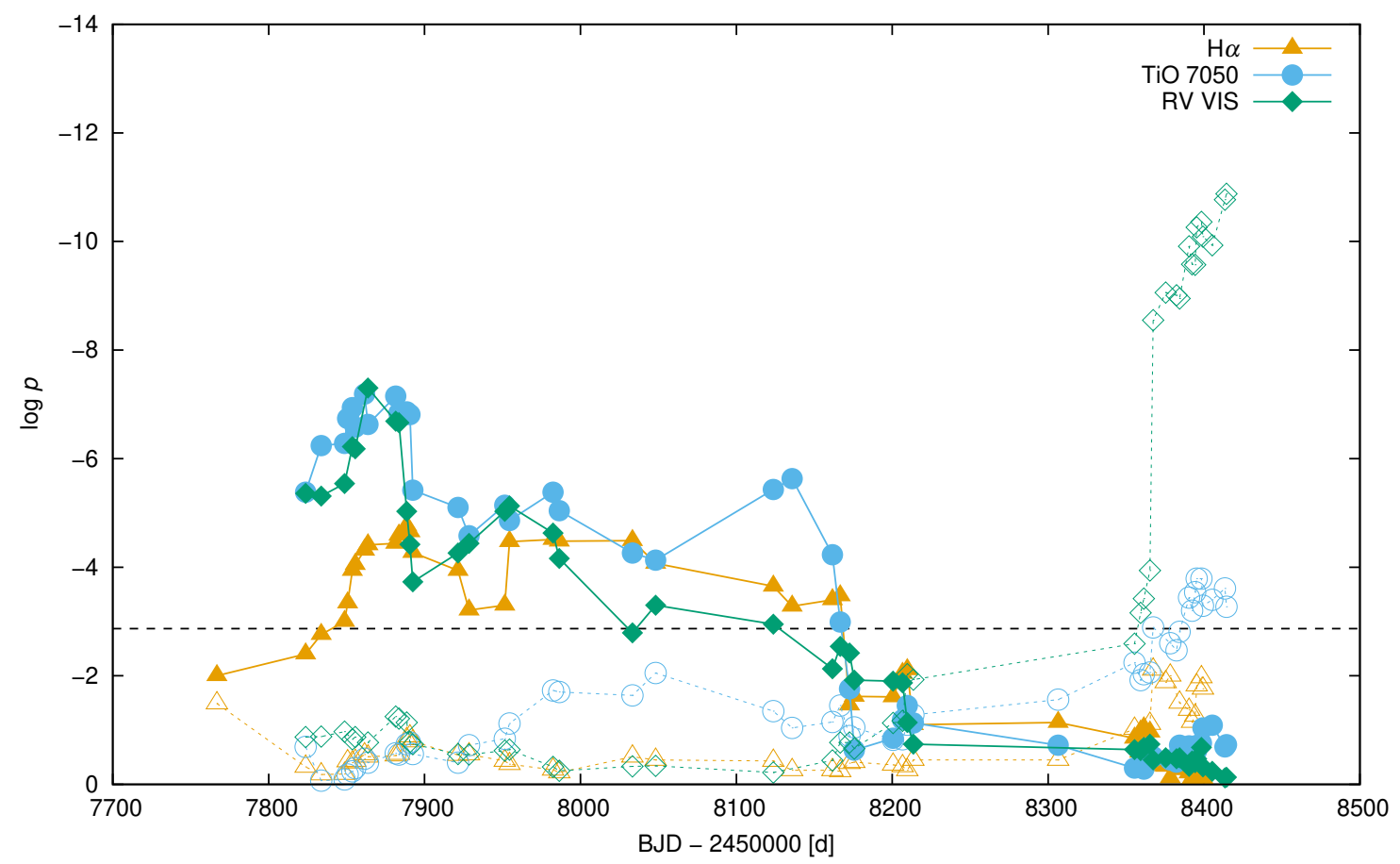

Figure 5.4.: Same as Fig. 5.2, but for TYC 3529-1437-1.

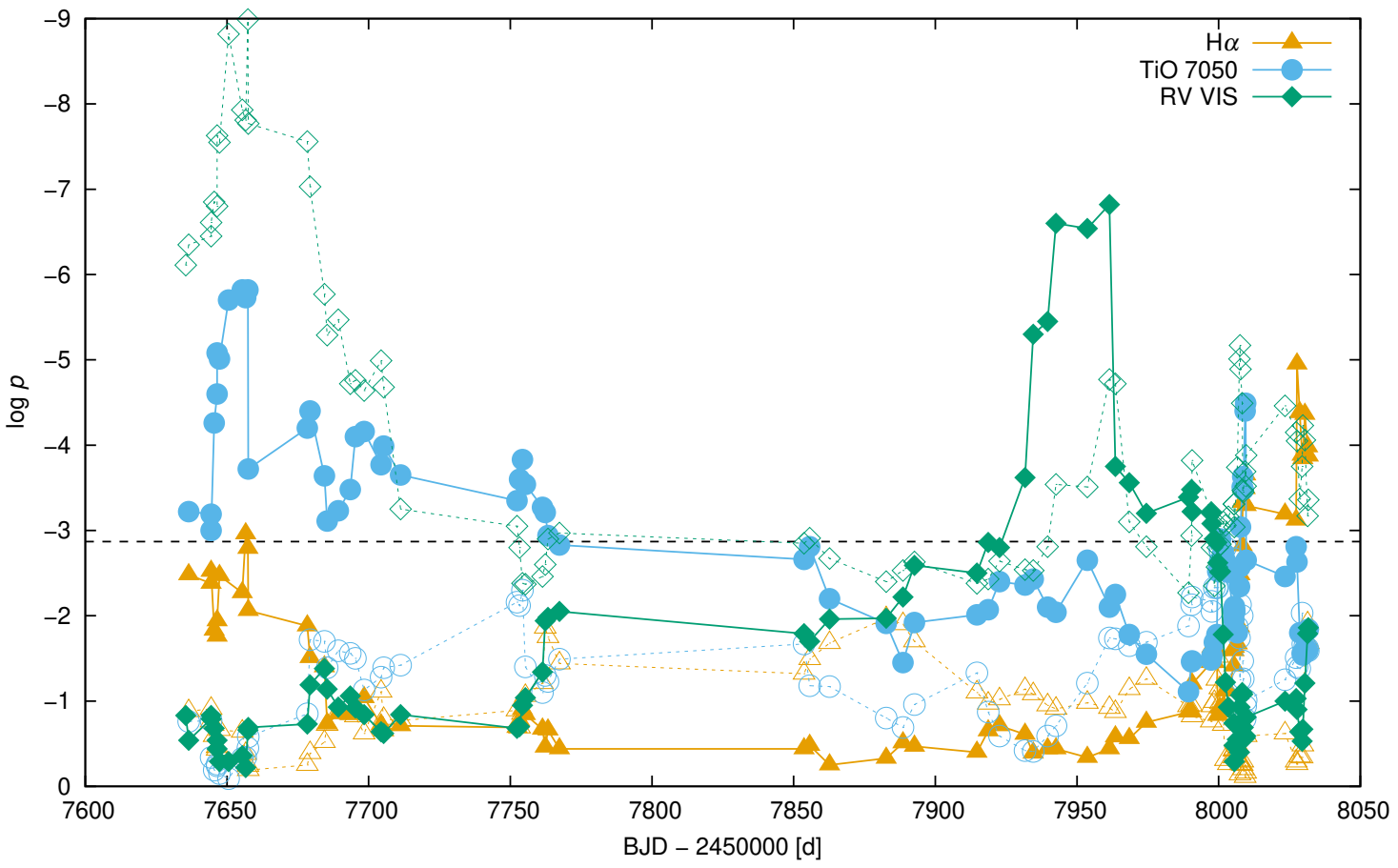

Figure 5.5.: Same as Fig. 5.2, but for EV Lac. 
A more varied behaviour is seen for the other two stars. For TYC 3529-1437-1, the three indicators in general show a significant signal at $1 / P_{\text {rot }}$ and no signal at $2 / P_{\text {rot }}$ in the first part of the time baseline. However, the signals at $1 / P_{\text {rot }}$ disappear as the subsets include more and more data points from observations after July 2018. Instead, a very significant signal in RV VIS and a barely significant signal in TiO 7050 appear at $2 / P_{\text {rot }}$. While $\log p\left(2 / P_{\text {rot }}\right)$ also increases in $\mathrm{H} \alpha$, the signal remains insignificant. In the case of EV Lac, significant signals are seen at $2 / P_{\text {rot }}$ in RV VIS and at $1 / P_{\text {rot }}$ in TiO 7050 in the first data subsets. Over time, these signals become less significant to insignificant, whereas a significant signal in RV VIS appears at $1 / P_{\text {rot }}$. This signal disappears again in the last data subsets, which include data points from observations with a higher cadence than once per night. However, the signal at $2 / P_{\text {rot }}$ in RV VIS is significant again in these subsets, and in some of these subsets significant signals appear at $1 / P_{\text {rot }}$ in $\mathrm{TiO} 7050$ and $\mathrm{H} \alpha$.

\subsection{Discussion}

Using GLS periodograms to iteratively determine three best-fit frequencies in each indicator for each star, I found in Sect. 5.1 that $\mathrm{TiO} 7050, \mathrm{H} \alpha$, Ca IRT-b, and $\mathrm{CaH} 3^{*}$ show the rotation frequency $1 / P_{\text {rot }}$ as one of the three best-fit frequencies for more than $10 \%$ of the stars with a known rotation period $P_{\text {rot }}>1$ d. In Schöfer et al. (2019), CaH 3* was not used, and $\mathrm{TiO} 8430$ also showed the rotation frequency as one of the best-fit frequencies for more than $10 \%$ of the stars with $P_{\text {rot }}>1 \mathrm{~d}$. Since July 2018, not only the number of sample stars and the total number of observations increased, but also more rotation periods were added to Carmencita. While the number of stars that show the rotation period in TiO 7050 remained constant at 19, the fraction therefore decreased, as there are now 148 stars with $P_{\text {rot }}>1$ d instead of 133. For most other indicators, the fraction also decreased. Fuhrmeister et al. (2019b) searched for rotation periods of 16 early-type stars of the sample in $\mathrm{H} \alpha$, Na D, and Ca IRT using different kinds of periodograms, and also found that $\mathrm{H} \alpha$ and $\mathrm{Ca}$ IRT are more likely to show the rotation period than $\mathrm{Na} \mathrm{D}$.

For 69 of 148 stars (47\%) with a known rotation period $P_{\text {rot }}>1 \mathrm{~d}$ the rotation frequency $1 / P_{\text {rot }}$ appears as one of the three best-fit frequencies in at least one indicator. This is true in more than two indicators for 18 stars (12\%). In Schöfer et al. (2019), 66 of 133 stars $(50 \%)$ showed the rotation period among the three best-fit frequencies in at least one indicator, and $15(11 \%)$ in more than two indicators. While more spectra, four more photospheric absorption band indices, and more rotation periods were added, the fractions of stars showing a signal remained almost constant. Of the 15 stars that showed rotational variation in more than two indicators in July 2018, the signal was lost for 4 stars. Of the seven stars that now show the rotation period in more than two indicators, but did not fulfil this criterion in July 2018, three stars did not have a known rotation period, one star was not in Carmencita, and one star shows the rotation period in the $\mathrm{CaH} 3^{*}$ index that was not considered previously. For the remaining two stars, only more spectra were added to the analysis. Three more stars would now fulfil the criteria to be included in 


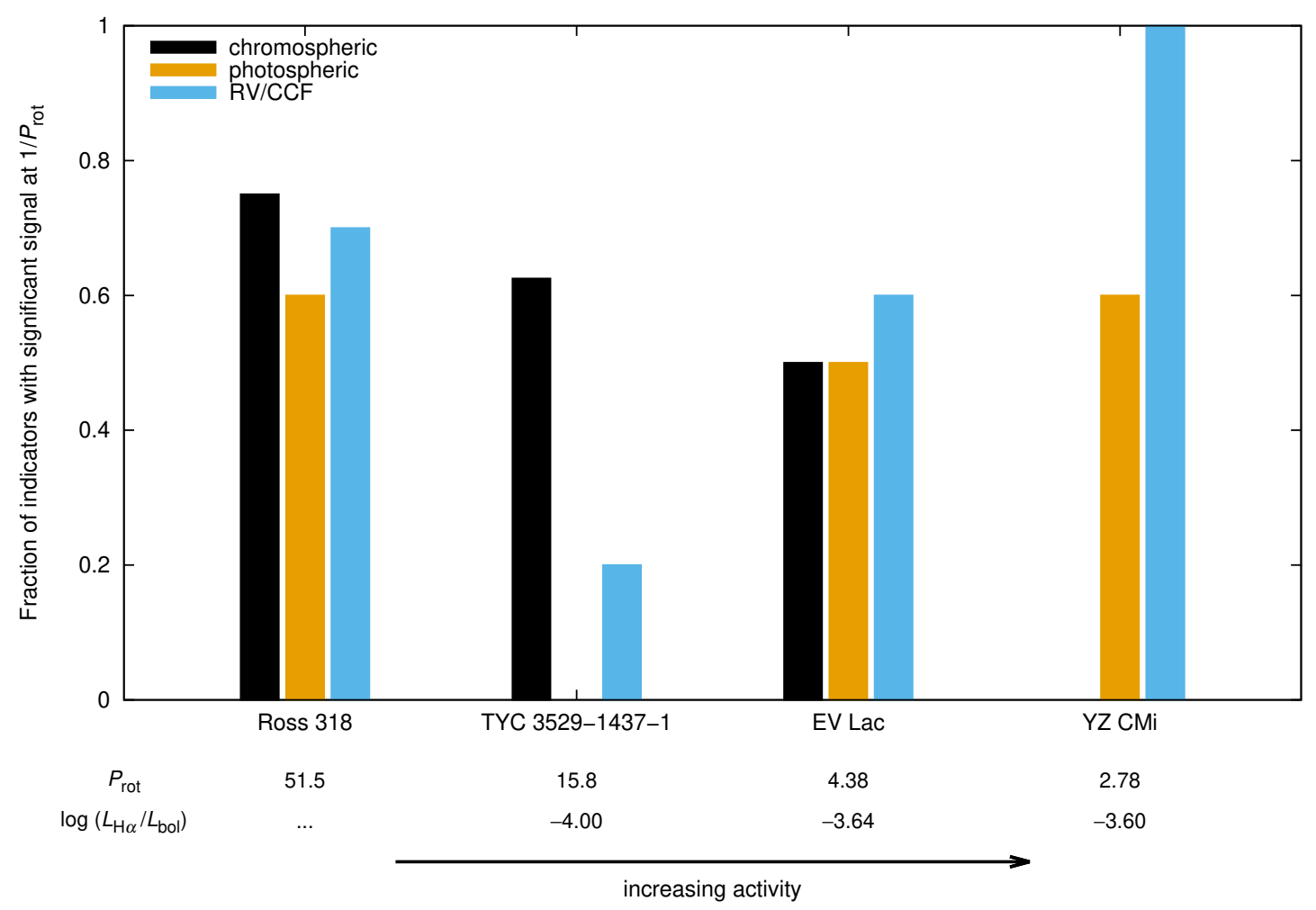

Figure 5.6.: Fractions of the eight chromospheric, the ten photospheric, and the ten RV or CCF indicators that show a significant signal at $1 / P_{\text {rot }}$ per star.

the more detailed analysis in Sect. 5.2: The low-active M1.0 star J07361-031 was now observed 41 times, the $\mathrm{H} \alpha$ inactive M4.0 star J10508+068 was now observed 47 times and has a known $P_{\text {rot }}=(64 \pm 19)$ d from Díez Alonso et al. (2019), and the $\mathrm{H} \alpha$ active M6.5 star J10482-113 was now observed 79 times and has a known $P_{\text {rot }}=(1.5 \pm 0.2) \mathrm{d}$ from Morin et al. (2010). As the rotation periods of the latter two stars have relatively large error bars, a large part of the frequency space is covered by the $3 \sigma$-confidence interval, and the best-fit frequencies of the different indicators do not agree with each other. In the case of J07361-031, however, the best-fit frequencies for $\mathrm{H} \alpha\left(0.0822 \mathrm{~d}^{-1}\right)$, Ca IRTa $\left(0.0821 \mathrm{~d}^{-1}\right)$, and Ca IRT-c $\left(0.0822 \mathrm{~d}^{-1}\right)$ are all in good agreement with the rotation frequency $1 / P_{\text {rot }}=(0.0820 \pm 0.0007) \mathrm{d}^{-1}$. No other indicator shows this frequency as one of the three best-fit frequencies.

The four stars analysed in detail in Sect. 5.2 do not have much in common. They cover diverse ranges of spectral subtypes, rotation periods, and normalised $\mathrm{H} \alpha$ luminosities. While they all show a significant signal at the rotation frequency in several indicators, the analysis revealed that there are also peculiarities in the rotation signals of each star. As a summary of the signals, I show a histogram of the fraction of the chromospheric (pEW's), photospheric (indices and $\mathrm{dLW}$ ), and RV or CCF indicators (CRX, RV, and all CCF parameters) per star in Fig. 5.6. 
RV VIS is the only indicator that shows a significant signal at $1 / P_{\text {rot }}$ for all four stars, whereas $\mathrm{He}_{3}, \mathrm{CaH} 2^{*}$, and VO 7942 do not show a significant signal at $1 / P_{\text {rot }}$ for any of the four stars. Although $\mathrm{H} \alpha$ inactive stars show only small variations in the chromospheric indicators and long rotation periods are hard to find (Fuhrmeister et al. 2019b), Ross 318 shows the rotation period in more indicators than the other three stars. Ross 318 is also the only of the four stars that shows a stronger signal in the dLW, CRX, CCF FWHM, and CCF BIS measured in the NIR channel, although there are fewer spectroscopic features in the NIR wavelength range (Reiners et al. 2018b) and therefore larger statistical fluctuations in the NIR parameters. As Zeeman broadening is wavelength-dependent, this could hint at an asymmetric magnetic field causing the variations. TYC 3529-1437-1 shows the fewest and weakest signals of the four stars. However, this is caused by a signal at $2 / P_{\text {rot }}$ that is introduced by the latest data. This is similar to photometric light curves, where the limited lifetime of active regions and differential rotations lead to one dip per rotation period, and at other times two dips per rotation period, as described in Sect. 1.4.1. In the TiO 7050 index, there is a clear trend towards lower values in the part of the data set that shows the signal at $2 / P_{\text {rot }}$. A lower TiO 7050 could be caused by more or larger starspots. Similarly, $\mathrm{pEW}_{\mathrm{H} \alpha}^{\prime}$ trends towards stronger excess emission. This also hints at a higher activity level of the star.

EV Lac has a higher normalised $\mathrm{H} \alpha$ luminosity and shows an even more complicated behaviour. In general, indicators that measure the strength of spectral lines ( $\mathrm{pEW}$ 's, photospheric band indices, $\mathrm{dLW}, \mathrm{CCF}$ contrast) show a stronger signal at $1 / P_{\text {rot }}$, while indicators that measure the position of spectral lines (CRX, RV) or a higher-order moment (CCF FWHM, CCF BIS) show a stronger signal at $2 / P_{\text {rot }}$ In RV VIS, EV Lac also shows the stronger signal at $1 / P_{\text {rot }}$ at some times and $2 / P_{\text {rot }}$ at other times. Photometric data published by Alekseev \& Kozhevnikova (2017) also show single-dip episodes and double-dip episodes for EV Lac. In contrast, YZ CMi does not show double-dip episodes in these data and also shows more stable signals at $1 / P_{\text {rot }}$ in most of the photospheric and RV or CCF parameters. EV Lac and YZ CMi have similar normalised $\mathrm{H} \alpha$ luminosities and magnetic field strengths (Shulyak et al. 2019), but EV Lac has a more complex magnetic topology according to Morin et al. (2008). YZ CMi is the only of the four stars that does not show a significant signal in any chromospheric indicator. While strong flaring events were discarded with a $2 \sigma$ clipping, there may still be non-periodic variations in the chromospheric indicators from observations during microflares or the decay of a strong flare, whereas the other indicators are less sensitive to these events (e.g. Zechmeister et al. 2009). Alternatively, the structures in the chromosphere might be distributed more homogeneously than the structures in the photosphere, leading to less rotational variation. 


\section{Summary and conclusion}

In this thesis, I presented $\mathrm{pEW}^{\prime}$ measurements of eight spectral lines with a chromospheric component using a spectral subtraction technique and indices of eight photospheric absorption bands to study the activity of $337 \mathrm{M}$ dwarfs in the CARMENES sample. To conclude the thesis, I now briefly summarise the results from Chapters 3, 4 and 5. For each of the topics covered by these three chapters, I also present an idea for future work.

Chromospheric activity indicators were measured after subtracting the spectrum of a reference star of the same spectral subtype. While this technique successfully removed photospheric lines from the line windows for $\mathrm{He}_{3}$ and $\mathrm{H} \alpha$, the line shapes of the photospheric components of the $\mathrm{Na} \mathrm{D}$ and Ca IRT lines were too different for stars of the same spectral type to be removed by the spectral subtraction. By defining stars with $\mathrm{pEW}_{\mathrm{H} \alpha}^{\prime}<-0.3 \AA$ as $\mathrm{H} \alpha$ active and the remaining stars as $\mathrm{H} \alpha$ inactive, I obtained fractions of $\mathrm{H} \alpha$ active stars per spectral subtype that agree with the results for similar samples of nearby M dwarfs (e.g. Reiners et al. 2012; West et al. 2015; Jeffers et al. 2018). The fraction increases drastically between spectral subtypes M3.5 and M4.0, where stars become fully convective (Stassun et al. 2011). I found a very strong correlation between $\mathrm{H} \alpha$ and $\mathrm{He}_{3}$, whereas correlations between $\mathrm{H} \alpha$ and $\mathrm{Na} \mathrm{D}$ or $\mathrm{H} \alpha$ and Ca IRT lines are disturbed by the remaining photospheric components of the latter lines and by different absolute levels of the pseudo-continuum flux. For $\mathrm{He} 10833$ and $\mathrm{Pa} \beta$, I found no correlation. I derived a relative variation of the $\mathrm{pEW}^{\prime}$ of each line for each star, and found that the relative $\mathrm{He}_{3}$ and $\mathrm{H} \alpha$ variations are anti-correlated with the normalised $\mathrm{H} \alpha$ luminosity, whereas the relative variations of $\mathrm{Na} \mathrm{D}$ and the $\mathrm{Ca}$ IRT lines are again biased by the photospheric components, and the relative variations of $\mathrm{He} 10833$ and $\mathrm{Pa} \beta$ are not useful because a division by a number close to zero is involved. Using a second set of reference stars for the spectral subtraction, I found that the results differ mostly be a constant offset that is larger for the lines with a strong photospheric component. Comparing the results with previous results for observations before July 2018 (Schöfer et al. 2019), I found that the method is stable. The results are also comparable with $\mathrm{pEW}$ values from Jeffers et al. (2018) that were derived with different spectrographs and methods. I also verified the relations between activity and rotation using the projected rotational velocities and the rotation periods listed in Table A.1, between activity and magnetic fields using the measurements by Shulyak et al. (2019), and between activity and age by calculating the fraction of $\mathrm{H} \alpha$ active stars per stellar population as given by Cortés-Contreras et al. (in prep.).

With the availability of spectra corrected for telluric absorption lines (Nagel et al. submitted), the previously discarded K I $\lambda 7700 \AA$ and Na I $\lambda 8200 \AA$ doublets (Robertson 
et al. 2016) could now be reconsidered. While most telluric lines were already removed from the co-added template spectra, now also useful measurements from individual observations would be available. However, these doublets have a photospheric component similar to $\mathrm{Na} \mathrm{D}$ and the Ca IRT, so the challenges in measuring those lines would also appear for these doublets.

For the photospheric absorption band indices, I found that they are all strongly correlated with each other. The scatter plots of different indices usually show different behaviours of $\mathrm{H} \alpha$ inactive and $\mathrm{H} \alpha$ active stars. While activity effects on photospheric bands were already described by Hawley et al. (1996) and Alonso-Floriano et al. (2015), their origin is not clear. To quantify these effects, I defined an inactivity line in the scatter plots and calculated the orthogonal distance of each star from this line. Colour-coding the scatter plots by metallicities from Schweitzer et al. (2019) revealed that the lowest metallicities are reported for very active stars. However, it is not plausible that the active stars are systematically metal-poor. The inactivity line and the orthogonal distances from it may help to better constrain the metallicity. Further, I found that the relative variations of the indices appear to be dominated by statistical fluctuations. Comparing again the results with previous results from Schöfer et al. (2019), I found no stability issue of the index measurement. The results are also comparable with similar indices from low-resolution spectroscopy.

The concept of the inactivity line can not only be improved with a better fit of this line. It would also be possible to calculate the indices for artificially broadened spectra of inactive stars. This way, isovelocity lines for a given rotational velocities could be derived. It might then be possible to disentangle the potential effects on the indices from rotational broadening and magnetic fields that were suggested by Schöfer et al. (2019).

Finally, the search for periodic variations of both the chromospheric and the photospheric indicators with the rotation period yielded a similar result as for the subset of observations before July 2018 that was analysed in Schöfer et al. (2019). While 18 of 148 stars with a known rotation period $P_{\text {rot }}>1 \mathrm{~d}$ show this period among the three best-fit frequencies for each indicator in more than two indicators. A periodic modulation with the rotation period is most commonly seen in TiO 7050, Ca IRT-b, $\mathrm{H} \alpha$, and $\mathrm{CaH} 3^{*}$. Although the four stars Ross 318, YZ CMi, TYC 3529-1437-1, and EV Lac all show significant signals in several indicators, a detailed analysis revealed that each star has its peculiarities, as their very different parameters suggest. Ross 318 shows stronger signals in some parameters derived from the NIR channel than in their VIS counterparts, although the information content in the VIS channel is higher. YZ CMi is a very active star and does not show any significant signal in the chromospheric indicators, but a very stable signal in photospheric indicators. TYC 3529-1437-1 transitioned from showing signals at $1 / P_{\text {rot }}$ to showing signals at $2 / P_{\text {rot }}$ EV Lac shows signals at $1 / P_{\text {rot }}$ in some indicators, but at $2 / P_{\text {rot }}$ in other indicators.

In the case of TYC 3529-1437-1, the new signals at $2 / P_{\text {rot }}$ led to a decreasing significance of the signals at $1 / P_{\text {rot }}$ in the periodograms of the full dataset. If other stars behave similarly, it is therefore possible that this remains unnoticed when only periodograms of 
full datasets are considered. Another approach is to compute rolling periodograms for all stars. However, this produces an enormous amount of data and it is likely that many times a signal at the rotation frequency or its harmonics appears by chance. 61 stars show a significant signal at least once at either $1 / P_{\text {rot }}$ or $2 / P_{\text {rot }}$ in any of the three indicators $\mathrm{H} \alpha$, TiO 7050, and RV VIS. With sophisticated criteria, it might be possible to automatically detect stars that show a behaviour similar to TYC 3529-1437-1. 



\section{A. Table of stars}

In the following Table A.1, I list the 337 stars of the CARMENES sample analysed in this thesis, their basic parameters used to calculate the results, the $\mathrm{pEW}_{\mathrm{H} \alpha}^{\prime}$ values derived from the co-added template spectra, and the normalised $\mathrm{H} \alpha$ luminosities. The following reference codes are used for the spectral types, projected rotational velocities, and rotation periods:

AF15: Alonso-Floriano et al. (2015); Benn17: Benneke et al. (2017); Bro10: Browning et al. (2010); DA19: Díez Alonso et al. (2019); Davi16: David et al. (2016); Del98: Delfosse et al. (1998); Fou18: Fouqué et al. (2018); Gig10: Gigoyan et al. (2010); Gra03: Gray et al. (2003); Gra06: Gray et al. (2006); HM15: Houdebine \& Mullan (2015); HMR14: Martínez-Rodríguez (2014); Jeff18: Jeffers et al. (2018); Jen09: Jenkins et al. (2009); Kir91: Kirkpatrick et al. (1991); Kira12: Kiraga (2012); KS07: Kiraga \& Stepien (2007); Lep03: Lépine et al. (2003); Lep13: Lépine et al. (2013); Mes11: Messina et al. (2011); Mor08: Morin et al. (2008); Mor10: Morin et al. (2010); New14: Newton et al. (2014); New16: Newton et al. (2016); PMSU: Palomar/Michigan State University Survey (Reid et al. 1995; Hawley et al. 1996); Rein18: Reiners et al. (2018b); Ria06: Riaz et al. (2006); Sch05: Scholz et al. (2005); Schm07: Schmidt et al. (2007); Shk09: Shkolnik et al. (2009); SM15: Suárez Mascareño et al. (2015); SM17: Suárez Mascareño et al. (2017); SM18: Suárez Mascareño et al. (2018); Tor06: Torres et al. (2006); Wat06: Watson et al. (2006); West15: West et al. (2015). 
Table A.1.: Identifications, common names, Gliese numbers, spectral types, projected rotational velocities, rotation periods, $\mathrm{pEW}_{\mathrm{H} \alpha}^{\prime}$ values, and normalised $\mathrm{H} \alpha$ luminosities of the analysed stars. Reference codes for $\mathrm{SpT}, v \sin i$, and $P_{\text {rot }}$ are defined in Appendix A.

\begin{tabular}{|c|c|c|c|c|c|c|c|c|c|c|c|}
\hline No. & Karmn & Name & Gl/GJ & SpT & Ref. & $\begin{array}{c}v \sin i \\
{\left[\mathrm{~km} \mathrm{~s}^{-1}\right]}\end{array}$ & Ref. & $\begin{array}{l}P_{\text {rot }} \\
\text { [d] }\end{array}$ & Ref. & $\begin{array}{c}\mathrm{pEW}_{\mathrm{H} \alpha}^{\prime} \\
{[\AA]}\end{array}$ & $\log \left(L_{\mathrm{H} \alpha} / L_{\mathrm{bol}}\right)$ \\
\hline 1 & $\mathrm{~J} 00051+457$ & GJ 2 & 2 & $\mathrm{M} 1.0 \mathrm{~V}$ & PMSU & $<2$ & Rein18 & $15.37 \pm 0.09$ & DA19 & $-0.077 \pm 0.007$ & $\ldots$ \\
\hline 2 & J00067-075 & GJ 1002 & 1002 & M5.5 V & PMSU & $<2$ & Rein18 & 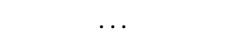 & & 0 & $\ldots$ \\
\hline 3 & J00162+198E & LP 404-062 & 1006B & $\mathrm{M} 4.0 \mathrm{~V}$ & AF15 & $<2$ & Rein18 & $105 \pm 44$ & DA19 & $0.053 \pm 0.011$ & $\ldots$ \\
\hline 4 & $\mathrm{~J} 00183+440$ & GX And & $15 \mathrm{~A}$ & $\mathrm{M} 1.0 \mathrm{~V}$ & AF15 & $<2$ & Rein18 & $45.0 \pm 4.4$ & SM18 & $-0.074 \pm 0.008$ & $\ldots$ \\
\hline 5 & $\mathrm{~J} 00184+440$ & GQ And & $15 \mathrm{~B}$ & $\mathrm{M} 3.5 \mathrm{~V}$ & PMSU & $<2$ & Rein18 & $\ldots$ & $\ldots$ & $-0.033 \pm 0.012$ & $\ldots$ \\
\hline 6 & J00286-066 & GJ 1012 & 1012 & $\mathrm{M} 4.0 \mathrm{~V}$ & PMSU & $<2$ & Rein18 & . & & $0.117 \pm 0.012$ & $\ldots$ \\
\hline 7 & $\mathrm{~J} 00389+306$ & Wolf 1056 & 26 & $\mathrm{M} 2.5 \mathrm{~V}$ & AF15 & $<2$ & Rein18 & $50.2 \pm 1.3$ & DA19 & $0.010 \pm 0.006$ & $\ldots$ \\
\hline 8 & $\mathrm{~J} 00570+450$ & G $172-030$ & $\ldots$ & $\mathrm{M} 3.0 \mathrm{~V}$ & Lep13 & $<2$ & Rein18 & $\ldots$ & & $-0.057 \pm 0.008$ & $\ldots$ \\
\hline 9 & $\mathrm{~J} 01013+613$ & GJ 47 & 47 & $\mathrm{M} 2.0 \mathrm{~V}$ & PMSU & $<2$ & Rein18 & $34.7 \pm 0.1$ & SM18 & $-0.079 \pm 0.009$ & . \\
\hline 10 & J01019+541 & G 218-020 & 3069 & M5.0 V & PMSU & $30.6 \pm 3.1$ & Rein18 & $0.2779 \pm 0.0006$ & DA19 & $-5.13 \pm 0.05$ & $-3.81 \pm 0.01$ \\
\hline 11 & $\mathrm{~J} 01025+716$ & Ross 318 & 48 & M3.0 V & PMSU & $<2$ & Rein18 & $51.5 \pm 2.6$ & DA19 & $0.072 \pm 0.015$ & $\ldots$ \\
\hline 12 & $\mathrm{~J} 01026+623$ & $\mathrm{BD}+61195$ & 49 & M1.5 V & AF15 & $<2$ & Rein18 & $19.9 \pm 0.4$ & DA19 & $-0.051 \pm 0.013$ & \\
\hline 13 & $\mathrm{~J} 01033+623$ & V388 Cas & 51 & M5.0 V & AF15 & $10.5 \pm 1.5$ & Rein18 & $1.02 \pm 0.01$ & DA19 & $-9.77 \pm 0.04$ & $-3.53 \pm 0.01$ \\
\hline 14 & J01048-181 & GJ 1028 & 1028 & M5.0 V & PMSU & $<2$ & Rein18 & $\ldots$ & $\ldots$ & $0.048 \pm 0.011$ & $\ldots$ \\
\hline 15 & J01125-169 & YZ Cet & 54.1 & $\mathrm{M} 4.5 \mathrm{~V}$ & PMSU & $3.4 \pm 0.8$ & Fou18 & $69.2 \pm 2.4$ & DA19 & $-1.35 \pm 0.04$ & $-4.34 \pm 0.02$ \\
\hline 16 & J01339-176 & LP 768-113 & $\ldots$ & $\mathrm{M} 4.0 \mathrm{~V}$ & Sch05 & $<2$ & Rein18 & $27.1 \pm 0.4$ & DA19 & $-1.640 \pm 0.028$ & $-4.17 \pm 0.01$ \\
\hline 17 & J01352-072 & Barta 16112 & $\ldots$ & $\mathrm{M} 4.0 \mathrm{~V}$ & Ria06 & $59.8 \pm 6.9$ & Rein18 & 0.7031 & Kira12 & $-7.81 \pm 0.06$ & $-3.49 \pm 0.01$ \\
\hline 18 & $\mathrm{~J} 01433+043$ & GJ 70 & 70 & $\mathrm{M} 2.0 \mathrm{~V}$ & PMSU & $<2$ & Rein18 & $\ldots$ & $\ldots$ & $-0.088 \pm 0.005$ & $\ldots$ \\
\hline 19 & $\mathrm{~J} 01518+644$ & G 244-037 & $3117 \mathrm{~A}$ & $\mathrm{M} 2.5 \mathrm{~V}$ & PMSU & $<2$ & Rein18 & $\ldots$ & $\ldots$ & $0.002 \pm 0.009$ & . \\
\hline 20 & $\mathrm{~J} 02002+130$ & TZ Ari & 83.1 & M3.5 V: & AF15 & $<2$ & Rein18 & $\ldots$ & $\ldots$ & $-2.048 \pm 0.026$ & $-4.04 \pm 0.01$ \\
\hline 21 & $\mathrm{~J} 02015+637$ & G 244-047 & 3126 & M3.0 V & PMSU & $<2$ & Rein18 & $\ldots$ & $\ldots$ & $0.056 \pm 0.009$ & .. \\
\hline 22 & $\mathrm{~J} 02070+496$ & G 173-037 & $\ldots$ & M3.5 V & Lep13 & $<2$ & Rein18 & $7.20 \pm 0.13$ & DA19 & $-0.505 \pm 0.023$ & $-4.64 \pm 0.02$ \\
\hline 23 & J02088+494 & G 173-039 & 3136 & M3.5 V & PMSU & $24.1 \pm 2.4$ & Rein18 & $0.74759 \pm 0.00019$ & DA19 & $-6.41 \pm 0.05$ & $-3.54 \pm 0.01$ \\
\hline 24 & $\mathrm{~J} 02123+035$ & $\mathrm{BD}+02348$ & 87 & M1.5 V & PMSU & $<2$ & Rein18 & $\ldots$ & $\ldots$ & $0.062 \pm 0.011$ & $\ldots$ \\
\hline 25 & $\mathrm{~J} 02222+478$ & $\mathrm{BD}+47612$ & 96 & $\mathrm{M} 0.5 \mathrm{~V}$ & PMSU & $<2$ & Rein18 & $29.5 \pm 0.4$ & DA19 & $0.050 \pm 0.004$ & \\
\hline 26 & $\mathrm{~J} 02336+249$ & GJ 102 & 102 & $\mathrm{M} 4.0 \mathrm{~V}$ & PMSU & $3.0 \pm 1.5$ & Rein18 & $\ldots$ & $\ldots$ & $-2.557 \pm 0.012$ & $-3.98 \pm 0.01$ \\
\hline 27 & J02358+202 & BD+19 381 & 104 & $\mathrm{M} 2.0 \mathrm{~V}$ & PMSU & $<2$ & Rein18 & $31.9 \pm 0.6$ & DA19 & $0.061 \pm 0.010$ & $\ldots$ \\
\hline 28 & $\mathrm{~J} 02362+068$ & BX Cet & 105B & $\mathrm{M} 4.0 \mathrm{~V}$ & AF15 & $<2$ & Rein18 & $\ldots$ & $\ldots$ & $0.073 \pm 0.010$ & $\ldots$ \\
\hline 29 & $\mathrm{~J} 02442+255$ & VX Ari & 109 & $\mathrm{M} 3.0 \mathrm{~V}$ & PMSU & $<2$ & Rein18 & $38.7 \pm 3.7$ & DA19 & $0.015 \pm 0.005$ & \\
\hline 30 & $\mathrm{~J} 02519+224$ & RBS 365 & $\ldots$ & $\mathrm{M} 4.0 \mathrm{~V}$ & Ria06 & $27.2 \pm 2.7$ & Rein18 & $0.85757 \pm 0.00003$ & DA19 & $-5.943 \pm 0.022$ & $-3.61 \pm 0.01$ \\
\hline 31 & $\mathrm{~J} 02530+168$ & Teegarden's Star & $\ldots$ & $\mathrm{M} 7.0 \mathrm{~V}$ & AF15 & $<2$ & Rein18 & $\ldots$ & $\ldots$ & $-0.49 \pm 0.04$ & $-5.37 \pm 0.03$ \\
\hline 32 & J02565+554W & Ross 364 & $119 \mathrm{~A}$ & M1.0 V & PMSU & $<2$ & Rein18 & $51.2 \pm 4.4$ & SM18 & $0.110 \pm 0.013$ & $\ldots$ \\
\hline 33 & $\mathrm{~J} 03133+047$ & CD Cet & 1057 & M5.0 V & PMSU & $<2$ & Rein18 & 126.2 & New16 & 0 & $\ldots$ \\
\hline 34 & $\mathrm{~J} 03181+382$ & HD 275122 & 134 & $\mathrm{M} 1.5 \mathrm{~V}$ & PMSU & $<2$ & Rein18 & $77.2 \pm 3.0$ & DA19 & $0.169 \pm 0.017$ & $\ldots$ \\
\hline 35 & $\mathrm{~J} 03213+799$ & GJ 133 & 133 & $\mathrm{M} 2.0 \mathrm{~V}$ & PMSU & $<2$ & Rein18 & $32.4 \pm 1.0$ & DA19 & $-0.040 \pm 0.013$ & $\ldots$ \\
\hline 36 & J03217-066 & G $077-046$ & 3218 & $\mathrm{M} 2.0 \mathrm{~V}$ & PMSU & $<2$ & Rein18 & $21.1 \pm 0.1$ & DA19 & $-0.320 \pm 0.015$ & $-4.73 \pm 0.02$ \\
\hline 37 & $\mathrm{~J} 03463+262$ & HD 23453 & 154 & M0.0 V & PMSU & $3.3 \pm 4.0$ & Rein18 & $\ldots$ & $\ldots$ & $-0.113 \pm 0.010$ & $\ldots$ \\
\hline
\end{tabular}


Table A.1.: Identifications, basic parameters, $\mathrm{pEW}_{\mathrm{H}_{\alpha}}^{\prime}$ values, and normalised $\mathrm{H} \alpha$ luminosities of the analysed stars (cont.)

\begin{tabular}{|c|c|c|c|c|c|c|c|c|c|c|c|}
\hline No. & Karmn & Name & $\mathrm{Gl} / \mathrm{GJ}$ & $\mathrm{SpT}$ & Ref. & $\begin{array}{c}v \sin i \\
{\left[\mathrm{~km} \mathrm{~s}^{-1}\right]}\end{array}$ & Ref. & $\begin{array}{l}P_{\text {rot }} \\
\text { [d] }\end{array}$ & Ref. & $\begin{array}{c}\mathrm{pEW}_{\mathrm{H} \alpha}^{\prime} \\
{[\AA]}\end{array}$ & $\log \left(L_{\mathrm{H} \alpha} / L_{\mathrm{bol}}\right)$ \\
\hline 38 & J03473-019 & G 080-021 & $\ldots$ & $\mathrm{M} 3.0 \mathrm{~V}$ & $\mathrm{AF} 15$ & $5.2 \pm 1.5$ & Rein18 & $3.87 \pm 0.01$ & DA19 & $-3.950 \pm 0.015$ & $-3.67 \pm 0.01$ \\
\hline 39 & $\mathrm{~J} 03531+625$ & Ross 567 & $\ldots$ & $\mathrm{M} 3.0 \mathrm{~V}$ & Lep13 & $<2$ & Rein 18 & $\ldots$ & $\ldots$ & $0.062 \pm 0.008$ & $\ldots$ \\
\hline 40 & J04153-076 & $o^{02}$ Eri C & $166 \mathrm{C}$ & $\mathrm{M} 4.5 \mathrm{~V}$ & AF15 & $2.1 \pm 1.5$ & Rein 18 & & & $-3.429 \pm 0.026$ & $-3.94 \pm 0.01$ \\
\hline 41 & $\mathrm{~J} 04173+088$ & LTT 11392 & 3270 & M $4.5 \mathrm{~V}$ & PMSU & 190.3 & Jeff18 & 0.1849 & West15 & & \\
\hline 42 & J04198+425 & LSR J0419+4233 & $\ldots$ & $\mathrm{M} 8.5 \mathrm{~V}$ & Lep03 & $3.6 \pm 2.3$ & Rein 18 & $\ldots$ & $\ldots$ & $-12.94 \pm 0.22$ & $-4.16 \pm 0.01$ \\
\hline 43 & $\mathrm{~J} 04219+213$ & LP 415-17 & $\ldots$ & M0.0 V & & $\ldots$ & & $\ldots$ & $\ldots$ & $0.10 \pm 0.05$ & $\ldots$ \\
\hline 44 & $\mathrm{~J} 04225+105$ & LSPM J0422+1031 & $\ldots$ & $\mathrm{M} 3.5 \mathrm{~V}$ & Lep13 & $<2$ & Rein 18 & $5.48 \pm 0.01$ & DA19 & $0.069 \pm 0.016$ & $\ldots$ \\
\hline 45 & $\mathrm{~J} 04290+219$ & $\mathrm{BD}+21652$ & 169 & $\mathrm{M} 0.5 \mathrm{~V}$ & Gra06 & $3.9 \pm 1.5$ & Rein 18 & $25.4 \pm 0.3$ & DA19 & $0.190 \pm 0.023$ & $\ldots$ \\
\hline 46 & $\mathrm{~J} 04311+589$ & STN 2051A & $169.1 \mathrm{~A}$ & $\mathrm{M} 4.0 \mathrm{~V}+$ & PMSU & $<1.9$ & Del98 & $\ldots$ & $\ldots$ & $-0.014 \pm 0.012$ & $\ldots$ \\
\hline 47 & J04376-110 & BD-11 916 & 173 & $\mathrm{M} 1.5 \mathrm{~V}$ & PMSU & $<2$ & Rein 18 & $\ldots$ & $\ldots$ & $0.064 \pm 0.013$ & $\ldots$ \\
\hline 48 & $\mathrm{~J} 04376+528$ & $\mathrm{BD}+52857$ & 172 & $\mathrm{M} 0.0 \mathrm{~V}$ & Gra03 & $3.4 \pm 1.5$ & Rein 18 & $\ldots$ & $\ldots$ & $-0.020 \pm 0.013$ & $\ldots$ \\
\hline 49 & $\mathrm{~J} 04429+189$ & HD 285968 & 176 & $\mathrm{M} 2.0 \mathrm{~V}$ & PMSU & $<2$ & Rein 18 & $40.7 \pm 0.4$ & DA19 & $0.001 \pm 0.009$ & $\ldots$ \\
\hline 50 & $\mathrm{~J} 04429+214$ & 2M J04425586+2128230 & $\ldots$ & $\mathrm{M} 3.5 \mathrm{~V}$ & Lep13 & $<2$ & Rein18 & $47.8 \pm 1.1$ & DA19 & $-0.043 \pm 0.015$ & \\
\hline 51 & $\mathrm{~J} 04472+206$ & RX J0447.2+2038 & $\ldots$ & $\mathrm{M} 5.0 \mathrm{~V}$ & AF15 & $47.6 \pm 26.8$ & Rein18 & $0.3426 \pm 0.0003$ & DA19 & $-9.84 \pm 0.05$ & $-3.53 \pm 0.01$ \\
\hline 52 & $\mathrm{~J} 04520+064$ & Wolf 1539 & 179 & $\mathrm{M} 3.5 \mathrm{~V}$ & PMSU & $<2$ & Rein 18 & $\ldots$ & $\ldots$ & $-0.049 \pm 0.014$ & $\ldots$ \\
\hline 53 & J04538-177 & GJ 180 & 180 & $\mathrm{M} 2.0 \mathrm{~V}$ & PMSU & $<2$ & Rein 18 & $\ldots$ & $\ldots$ & $0.027 \pm 0.010$ & $\ldots$ \\
\hline 54 & $\mathrm{~J} 04588+498$ & $\mathrm{BD}+491280$ & 181 & $\mathrm{M} 0.0 \mathrm{~V}$ & PMSU & $<2$ & Rein 18 & $\ldots$ & $\ldots$ & $-0.074 \pm 0.007$ & \\
\hline 55 & J05019-069 & LP $656-038$ & 3323 & M $4.0 \mathrm{~V}$ & PMSU & $3.8 \pm 1.3$ & Fou18 & 88.5 & Kira12 & $-0.67 \pm 0.04$ & $-4.56 \pm 0.03$ \\
\hline 56 & $\mathrm{~J} 05019+011$ & 1RXS J050156.7+010845 & & $\mathrm{M} 4.0 \mathrm{~V}$ & $\mathrm{AF} 15$ & $6.5 \pm 1.5$ & Rein 18 & $2.12 \pm 0.02$ & DA19 & $-6.379 \pm 0.019$ & $-3.58 \pm 0.01$ \\
\hline 57 & J05033-173 & LP 776-046 & 3325 & $\mathrm{M} 3.0 \mathrm{~V}$ & PMSU & $<2$ & Rein18 & & & $0.019 \pm 0.006$ & \\
\hline 58 & $\mathrm{~J} 05062+046$ & RX J0506.2+0439 & $\ldots$ & $\mathrm{M} 4.0 \mathrm{~V}$ & AF15 & $24.9 \pm 2.5$ & Rein18 & $0.8650 \pm 0.0006$ & DA19 & $-7.22 \pm 0.04$ & $-3.53 \pm 0.01$ \\
\hline 59 & J05084-210 & 2M J05082729-2101444 & & M5.0 V & Ria06 & $25.2 \pm 2.5$ & Rein 18 & $\ldots$ & $\ldots$ & $-15.77 \pm 0.08$ & $-3.33 \pm 0.01$ \\
\hline 60 & $\mathrm{~J} 05127+196$ & GJ 192 & 192 & $\mathrm{M} 2.0 \mathrm{~V}$ & PMSU & $<2$ & Rein 18 & $\ldots$ & $\ldots$ & $0.038 \pm 0.012$ & $\ldots$ \\
\hline 61 & $\mathrm{~J} 05280+096$ & Ross 41 & 203 & $\mathrm{M} 3.5 \mathrm{~V}$ & PMSU & $<2$ & Rein 18 & $17.2 \pm 0.1$ & DA19 & $-0.038 \pm 0.009$ & $\ldots$ \\
\hline 62 & J05314-036 & HD 36395 & 205 & M1.5 V & AF15 & $<2$ & Rein18 & $33.8 \pm 0.6$ & DA19 & $0.101 \pm 0.019$ & \\
\hline 63 & $\mathrm{~J} 05337+019$ & V371 Ori & 207.1 & $\mathrm{M} 2.5 \mathrm{~V}$ & PMSU & $9.8 \pm 1.5$ & Rein 18 & $2.81 \pm 0.25$ & HM15 & $-5.03 \pm 0.04$ & $-3.55 \pm 0.01$ \\
\hline 64 & $\mathrm{~J} 05348+138$ & Ross 46 & 3356 & $\mathrm{M} 3.5 \mathrm{~V}$ & PMSU & $<2$ & Rein18 & $\ldots$ & $\ldots$ & $0.016 \pm 0.014$ & $\ldots$ \\
\hline 65 & J05360-076 & Wolf 1457 & 3357 & $\mathrm{M} 4.0 \mathrm{~V}$ & PMSU & $<2$ & Rein18 & $\ldots$ & $\ldots$ & $0.061 \pm 0.013$ & $\ldots$ \\
\hline 66 & $\mathrm{~J} 05365+113$ & V2689 Ori & 208 & $\mathrm{M} 0.0 \mathrm{~V}$ & Lep13 & $3.8 \pm 1.5$ & Rein18 & $12.3 \pm 0.1$ & DA19 & $-0.447 \pm 0.012$ & $-4.53 \pm 0.02$ \\
\hline 67 & $\mathrm{~J} 05366+112$ & 2M J05363846+11117487 & $\ldots$ & $\mathrm{M} 4.0 \mathrm{~V}$ & Lep13 & $2.4 \pm 1.5$ & Rein 18 & $\ldots$ & $\ldots$ & $-2.878 \pm 0.021$ & $-3.92 \pm 0.01$ \\
\hline 68 & $\mathrm{~J} 05394+406$ & LSR J0539+4038 & $\ldots$ & M8.0 V & Lep03 & $4.1 \pm 1.5$ & Rein 18 & $\ldots$ & $\ldots$ & $-4.81 \pm 0.12$ & $-4.50 \pm 0.02$ \\
\hline 69 & $\mathrm{~J} 05415+534$ & HD 233153 & 212 & $\mathrm{M} 1.0 \mathrm{~V}$ & $\mathrm{AF} 15$ & $<2$ & Rein 18 & $\ldots$ & $\ldots$ & $-0.056 \pm 0.010$ & $\ldots$ \\
\hline 70 & $\mathrm{~J} 05421+124$ & V1352 Ori & 213 & $\mathrm{M} 4.0 \mathrm{~V}$ & $\mathrm{AF} 15$ & $<2$ & Rein 18 & $\ldots$ & $\ldots$ & $0.069 \pm 0.010$ & $\ldots$ \\
\hline 71 & $\mathrm{~J} 06000+027$ & G 099-049 & 3379 & $\mathrm{M} 4.0 \mathrm{~V}$ & PMSU & $4.9 \pm 1.5$ & Rein18 & $1.81 \pm 0.01$ & DA19 & $-2.710 \pm 0.018$ & $-3.95 \pm 0.01$ \\
\hline 72 & $\mathrm{~J} 06011+595$ & G 192-013 & 3378 & $\mathrm{M} 3.5 \mathrm{~V}$ & PMSU & $<2$ & Rein18 & $\ldots$ & $\ldots$ & $-0.033 \pm 0.009$ & $\ldots$ \\
\hline 73 & $\mathrm{~J} 06024+498$ & G 192-015 & 3380 & M5.0 V & AF15 & $<2$ & Rein18 & $105 \pm 6$ & DA19 & $0.025 \pm 0.014$ & $\ldots$ \\
\hline 74 & $\mathrm{~J} 06103+821$ & GJ 226 & 226 & $\mathrm{M} 2.0 \mathrm{~V}$ & PMSU & $<2$ & Rein 18 & $44.6 \pm 1.0$ & DA19 & 0 & $\ldots$ \\
\hline 75 & J06105-218 & $\mathrm{HD} 42581 \mathrm{~A}$ & $229 \mathrm{~A}$ & $\mathrm{M} 0.5 \mathrm{~V}$ & PMSU & $<2$ & Rein 18 & $27.3 \pm 0.2$ & DA19 & $0.000 \pm 0.009$ & $\ldots$ \\
\hline 76 & $\mathrm{~J} 06246+234$ & Ross 64 & 232 & $\mathrm{M} 4.0 \mathrm{~V}$ & AF15 & $<2$ & Rein18 & & $\ldots$ & $0.041 \pm 0.014$ & $\ldots$ \\
\hline 77 & J06318+414 & LP 205-044 & 3396 & M $5.0 \mathrm{~V}$ & PMSU & $58.4 \pm 26.1$ & Rein 18 & $0.29952 \pm 0.00007$ & DA19 & $\ldots$ & $\ldots$ \\
\hline
\end{tabular}


Table A.1.: Identifications, basic parameters, $\mathrm{pEW}_{\mathrm{H}}^{\prime}$ values, and normalised $\mathrm{H} \alpha$ luminosities of the analysed stars (cont.)

\begin{tabular}{|c|c|c|c|c|c|c|c|c|c|c|c|}
\hline No. & Karmn & Name & $\mathrm{Gl} / \mathrm{GJ}$ & SpT & Ref. & $\begin{array}{c}v \sin i \\
{\left[\mathrm{~km} \mathrm{~s}^{-1}\right]}\end{array}$ & Ref. & $\begin{array}{l}P_{\text {rot }} \\
{[\mathrm{d}]}\end{array}$ & Ref. & $\begin{array}{c}\mathrm{pEW}_{\mathrm{H} \alpha}^{\prime} \\
{[\AA]}\end{array}$ & $\log \left(L_{\mathrm{H} \alpha} / L_{\mathrm{bol}}\right)$ \\
\hline 78 & $\mathrm{~J} 06371+175$ & HD 260655 & 239 & $\mathrm{M} 0.0 \mathrm{~V}$ & Lep13 & $<2$ & Rein18 & $\ldots$ & $\ldots$ & $-0.061 \pm 0.021$ & $\ldots$ \\
\hline 79 & J06396-210 & LP 780-032 & & $\mathrm{M} 4.0 \mathrm{~V}$ & Sch05 & $<2$ & Rein18 & 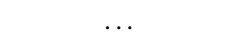 & & $-0.134 \pm 0.020$ & $\ldots$ \\
\hline 80 & $\mathrm{~J} 06421+035$ & G 108-021 & $3404 \mathrm{~A}$ & M3.5 V & PMSU & $<2$ & Rein18 & $83.4 \pm 7$ & DA19 & $0.039 \pm 0.013$ & $\ldots$ \\
\hline 81 & $\mathrm{~J} 06548+332$ & Wolf 294 & 251 & $\mathrm{M} 3.0 \mathrm{~V}$ & $\mathrm{AF} 15$ & $<2$ & Rein 18 & $18.1 \pm 0.3$ & DA19 & $0.018 \pm 0.009$ & \\
\hline 82 & $\mathrm{~J} 06574+740$ & 2M J06572616+7405265 & & $\mathrm{M} 4.0 \mathrm{~V}$ & Lep13 & $27.1 \pm 2.7$ & Rein 18 & $\ldots$ & $\ldots$ & $-5.49 \pm 0.05$ & $-3.64 \pm 0.01$ \\
\hline 83 & J06594+193 & GJ 1093 & 1093 & $\mathrm{M} 5.0 \mathrm{~V}$ & PMSU & $<2$ & Rein18 & $\ldots$ & $\ldots$ & $-0.279 \pm 0.024$ & \\
\hline 84 & J07001-190 & 2M J07000682-1901235 & $\ldots$ & $\mathrm{M} 5.0 \mathrm{~V}$ & $\mathrm{AF} 15$ & $3.8 \pm 1.5$ & Rein 18 & $\ldots$ & $\ldots$ & $-7.05 \pm 0.03$ & $-3.68 \pm 0.01$ \\
\hline 85 & $\mathrm{~J} 07033+346$ & LP 255-011 & 3423 & $\mathrm{M} 4.0 \mathrm{~V}$ & PMSU & $<2$ & Rein 18 & $8.04 \pm 0.03$ & DA19 & $-3.005 \pm 0.028$ & $-3.91 \pm 0.01$ \\
\hline 86 & $\mathrm{~J} 07044+682$ & GJ 258 & 258 & $\mathrm{M} 3.0 \mathrm{~V}$ & PMSU & $<2$ & Rein18 & & & $0.084 \pm 0.009$ & $\ldots$ \\
\hline 87 & $\mathrm{~J} 07274+052$ & Luyten's Star & 273 & M3.5 V & AF15 & $<2$ & Rein18 & $93.5 \pm 16$ & SM17 & $-0.042 \pm 0.015$ & $\ldots$ \\
\hline 88 & J07287-032 & GJ 1097 & 1097 & M3.0 V & PMSU & $<2$ & Rein18 & $\ldots$ & . & $0.040 \pm 0.008$ & 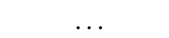 \\
\hline 89 & $\mathrm{~J} 07319+362 \mathrm{~N}$ & BL Lyn & $277 \mathrm{C}$ & M3.5 V & AF15 & $<2$ & Rein 18 & $16.4 \pm 0.3$ & DA19 & $-2.727 \pm 0.018$ & $-3.91 \pm 0.01$ \\
\hline 90 & $\mathrm{~J} 07353+548$ & GJ 3452 & 3452 & $\mathrm{M} 2.0 \mathrm{~V}$ & PMSU & $<2$ & Rein18 & $21.8 \pm 1.0$ & DA19 & $-0.034 \pm 0.009$ & $\ldots$ \\
\hline 91 & J07361-031 & BD-02 2198 & $282 \mathrm{C}$ & $\mathrm{M} 1.0 \mathrm{~V}$ & $\mathrm{AF} 15$ & $3.1 \pm 1.5$ & Rein 18 & $12.2 \pm 0.1$ & DA19 & $-0.867 \pm 0.014$ & $-4.28 \pm 0.01$ \\
\hline 92 & J07386-212 & LP 763-001 & 3459 & M3.0 V & PMSU & $<2$ & Rein 18 & $\ldots$ & $\ldots$ & $0.033 \pm 0.009$ & $\ldots$ \\
\hline 93 & $\mathrm{~J} 07393+021$ & BD+02 1729 & 281 & $\mathrm{M} 0.0 \mathrm{~V}$ & PMSU & $<2$ & Rein18 & $\ldots$ & $\ldots$ & $0.005 \pm 0.007$ & $\ldots$ \\
\hline 94 & J07403-174 & LP 783-002 & $283 \mathrm{~B}$ & $\mathrm{M} 6.0 \mathrm{~V}$ & PMSU & $<2$ & Rein18 & & & 0 & 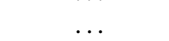 \\
\hline 95 & $\mathrm{~J} 07446+035$ & $\mathrm{YZCMi}$ & 285 & $\mathrm{M} 4.5 \mathrm{~V}$ & PMSU & $4.0 \pm 1.5$ & Rein 18 & $2.78 \pm 0.01$ & DA19 & $-7.386 \pm 0.023$ & $-3.60 \pm 0.01$ \\
\hline 96 & $\mathrm{~J} 07472+503$ & 2M J07471385+5020386 & & $\mathrm{M} 4.0 \mathrm{~V}$ & Lep13 & $10.1 \pm 1.5$ & Rein 18 & $1.32 \pm 0.01$ & DA19 & $-3.812 \pm 0.024$ & $-3.80 \pm 0.01$ \\
\hline 97 & $\mathrm{~J} 07558+833$ & GJ 1101 & 1101 & $\mathrm{M} 4.5 \mathrm{~V}:$ & AF15 & $12.1 \pm 1.5$ & Rein18 & $1.11 \pm 0.01$ & DA19 & $-5.257 \pm 0.029$ & $-3.75 \pm 0.01$ \\
\hline 98 & $\mathrm{~J} 07582+413$ & GJ 1105 & 1105 & M3.5 V & PMSU & $<2$ & Rein 18 & $\ldots$ & $\ldots$ & $-0.029 \pm 0.011$ & $\ldots$ \\
\hline 99 & $\mathrm{~J} 08023+033$ & G 050-016 A & 3473 & $\mathrm{M} 4.0 \mathrm{~V}$ & PMSU & & & & & $0.137 \pm 0.014$ & $\ldots$ \\
\hline 100 & $\mathrm{~J} 08119+087$ & Ross 619 & 299 & $\mathrm{M} 4.5 \mathrm{~V}$ & PMSU & $3.0 \pm 1.7$ & Del98 & $5.42 \pm 0.15$ & DA19 & $-0.02 \pm 0.04$ & $\ldots$ \\
\hline 101 & J08126-215 & GJ 300 & 300 & $\mathrm{M} 4.0 \mathrm{~V}$ & PMSU & $<2$ & Rein18 & & & $0.021 \pm 0.016$ & $\ldots$ \\
\hline 102 & $\mathrm{~J} 08161+013$ & GJ 2066 & 2066 & $\mathrm{M} 2.0 \mathrm{~V}$ & AF15 & $<2$ & Rein18 & $40.7 \pm 0.4$ & DA19 & $0.010 \pm 0.007$ & $\ldots$ \\
\hline 103 & $\mathrm{~J} 08293+039$ & 2M J08292191+0355092 & & $\mathrm{M} 2.5 \mathrm{~V}$ & Lep13 & $<2$ & Rein 18 & & & $0.040 \pm 0.011$ & \\
\hline 104 & $\mathrm{~J} 08298+267$ & DX Cnc & 1111 & $\mathrm{M} 6.5 \mathrm{~V}$ & AF15 & $10.5 \pm 1.5$ & Rein18 & $0.45900 \pm 0.00001$ & DA19 & $-5.15 \pm 0.07$ & $-4.27 \pm 0.01$ \\
\hline 105 & $\mathrm{~J} 08315+730$ & LP 035-219 & $\ldots$ & $\mathrm{M} 4.0 \mathrm{~V}$ & Lep13 & $<2$ & Rein18 & $105 \pm 11$ & DA19 & $0.074 \pm 0.011$ & $\ldots$ \\
\hline 106 & $\mathrm{~J} 08358+680$ & G 234-037 & 3506 & M2.5 V & PMSU & $<2$ & Rein 18 & & & $-0.207 \pm 0.009$ & $\ldots$ \\
\hline 107 & $\mathrm{~J} 08402+314$ & LSPM J0840+3127 & $\ldots$ & M3.5 V & Lep13 & $<2$ & Rein18 & $118 \pm 14$ & DA19 & $0.021 \pm 0.010$ & $\ldots$ \\
\hline 108 & J08409-234 & LP 844-008 & 317 & M3.5 V & Lep13 & $<2.5$ & Brol0 & $\ldots$ & $\ldots$ & $0.012 \pm 0.013$ & \\
\hline 109 & $\mathrm{~J} 08413+594$ & LP 090-018 & 3512 & M5.5 V & PMSU & $<2$ & Rein 18 & $\ldots$ & $\ldots$ & $-1.370 \pm 0.012$ & $-4.44 \pm 0.01$ \\
\hline 110 & $\mathrm{~J} 08526+283$ & rho $\mathrm{Cnc} B$ & $324 \mathrm{~B}$ & $\mathrm{M} 4.5 \mathrm{~V}$ & AF15 & $<2$ & Rein 18 & $\ldots$ & $\ldots$ & $0.047 \pm 0.011$ & $\ldots$ \\
\hline 111 & J08536-034 & LP 666-009 & 3517 & $\mathrm{M} 9.0 \mathrm{~V}$ & Jen09 & $9.3 \pm 2.8$ & Rein18 & & & & \\
\hline 112 & $\mathrm{~J} 09003+218$ & LP $368-128$ & $\ldots$ & M6.5 V & $\mathrm{AF} 15$ & $14.3 \pm 1.5$ & Rein 18 & 0.439 & New16 & $-6.88 \pm 0.06$ & $-4.15 \pm 0.01$ \\
\hline 113 & $\mathrm{~J} 09005+465$ & GJ 1119 & 1119 & M $4.5 \mathrm{~V}$ & PMSU & $<2$ & Rein 18 & 8 & West15 & $-0.970 \pm 0.013$ & $-4.49 \pm 0.01$ \\
\hline 114 & $\mathrm{~J} 09028+680$ & LP 060-179 & 3526 & $\mathrm{M} 4.0 \mathrm{~V}$ & PMSU & $<2$ & Rein18 & $\ldots$ & $\ldots$ & $0.079 \pm 0.011$ & \\
\hline 115 & $\mathrm{~J} 09033+056$ & NLTT 20861 & $\ldots$ & $\mathrm{M} 7.0 \mathrm{~V}$ & New14 & $9.7 \pm 1.5$ & Rein 18 & $\ldots$ & $\ldots$ & $-3.70 \pm 0.09$ & $-4.49 \pm 0.01$ \\
\hline 116 & $\mathrm{~J} 09133+688$ & G 234-057 & $\ldots$ & $\mathrm{M} 2.5 \mathrm{~V}$ & Lep13 & $<2$ & Rein18 & $10.4 \pm 0.1$ & SM18 & $-0.565 \pm 0.017$ & $-4.50 \pm 0.02$ \\
\hline 117 & J09140+196 & LP 427-016 & $\ldots$ & $\mathrm{M} 3.0 \mathrm{~V}$ & Lep13 & $<2$ & Rein 18 & $89.9 \pm 2$ & DA19 & $-0.148 \pm 0.010$ & $\ldots$ \\
\hline
\end{tabular}


Table A.1.: Identifications, basic parameters, $\mathrm{pEW}_{\mathrm{H}_{\alpha}}^{\prime}$ values, and normalised $\mathrm{H} \alpha$ luminosities of the analysed stars (cont.)

\begin{tabular}{|c|c|c|c|c|c|c|c|c|c|c|c|}
\hline No. & Karmn & Name & $\mathrm{Gl} / \mathrm{GJ}$ & SpT & Ref. & $\begin{array}{c}v \sin i \\
{\left[\mathrm{~km} \mathrm{~s}^{-1}\right]}\end{array}$ & Ref. & $\begin{array}{l}P_{\text {rot }} \\
\text { [d] }\end{array}$ & Ref. & $\begin{array}{c}\mathrm{pEW} \\
{[\AA \AA \mathrm{H} \alpha} \\
\end{array}$ & $\log \left(L_{\mathrm{H} \alpha} / L_{\mathrm{bol}}\right)$ \\
\hline 118 & $\mathrm{~J} 09143+526$ & HD 79210 & $338 \mathrm{~A}$ & M0.0 V & AF15 & $2.9 \pm 1.2$ & Del98 & $\ldots$ & $\ldots$ & $-0.027 \pm 0.012$ & $\cdots$ \\
\hline 119 & J09144+526 & HD 79211 & $338 \mathrm{~B}$ & M0.0 V & AF15 & $2.3 \pm 1.5$ & Rein18 & $\ldots$ & $\ldots$ & $-0.027 \pm 0.01$ & \\
\hline 120 & J09161+018 & RX J0916.1+0153 & $\ldots$ & $\mathrm{M} 4.0 \mathrm{~V}$ & Lep13 & $10.4 \pm 1.5$ & Rein18 & $\ldots$ & $\ldots$ & $-5.066 \pm 0.029$ & $-3.68 \pm 0.01$ \\
\hline 121 & J09163-186 & LP 787-052 & 3543 & M1.5 V & PMSU & $<2$ & Rein18 & $\ldots$ & $\ldots$ & $-0.378 \pm 0.012$ & $-4.65 \pm 0.02$ \\
\hline 122 & $\mathrm{~J} 09307+003$ & GJ 1125 & 1125 & M3.5 V & PMSU & $<2$ & Rein18 & $\ldots$ & $\ldots$ & $-0.004 \pm 0.012$ & $\ldots$ \\
\hline 123 & J09360-216 & GJ 357 & 357 & M2.5 V & PMSU & $2.5 \pm 1.1$ & Fou18 & $74.3 \pm 1.7$ & SM15 & $-0.027 \pm 0.012$ & $\ldots$ \\
\hline 124 & $\mathrm{~J} 09411+132$ & Ross 85 & 361 & M1.5 V & PMSU & $<2$ & Rein18 & $\ldots$ & $\ldots$ & $0.005 \pm 0.011$ & $\ldots$ \\
\hline 125 & J09423+559 & GJ 363 & 363 & M3.5 V & PMSU & $<2$ & Rein18 & $74.3 \pm 2.8$ & DA19 & $-0.029 \pm 0.015$ & \\
\hline 126 & $\mathrm{~J} 09425+700$ & GJ 360 & 360 & $\mathrm{M} 2.0 \mathrm{~V}$ & PMSU & $<2$ & Rein18 & $21 \pm 0.4$ & DA19 & $-0.598 \pm 0.009$ & $-4.45 \pm 0.01$ \\
\hline 127 & $\mathrm{~J} 09428+700$ & GJ 362 & 362 & M3.0 V & PMSU & $<2$ & Rein18 & $23.9 \pm 0.6$ & DA19 & $-0.775 \pm 0.013$ & $-4.38 \pm 0.01$ \\
\hline 128 & $\mathrm{~J} 09439+269$ & Ross 93 & 3564 & M3.5 V & PMSU & $<2$ & Rein18 & $13.7 \pm 0.9$ & DA19 & $0.069 \pm 0.016$ & $\ldots$ \\
\hline 129 & J09447-182 & GJ 1129 & 1129 & $\mathrm{M} 4.0 \mathrm{~V}$ & PMSU & $<2$ & Rein18 & $\ldots$ & $\ldots$ & $0.088 \pm 0.012$ & \\
\hline 130 & J09449-123 & G 161-071 & & M5.0 V & AF15 & $31.2 \pm 3.1$ & Rein18 & $\ldots$ & $\ldots$ & $-14.08 \pm 0.08$ & $-3.38 \pm 0.01$ \\
\hline 131 & $\mathrm{~J} 09468+760$ & BD+76 3952 & 366 & $\mathrm{M} 1.5 \mathrm{~V}$ & PMSU & $<2$ & Rein18 & $\ldots$ & $\ldots$ & $0.116 \pm 0.014$ & $\ldots$ \\
\hline 132 & J09511-123 & BD-11 2741 & 369 & M0.5 V & PMSU & $<2$ & Rein18 & $\ldots$ & $\ldots$ & $-0.022 \pm 0.013$ & $\ldots$ \\
\hline 133 & J09561+627 & $\mathrm{BD}+63869$ & 373 & M0.0 V & PMSU & $<2$ & Rein18 & $\ldots$ & $\ldots$ & $-0.078 \pm 0.006$ & $\ldots$ \\
\hline 134 & $\mathrm{~J} 10023+480$ & BD+48 1829 & 378 & $\mathrm{M} 1.0 \mathrm{~V}$ & PMSU & $<2$ & Rein18 & $\ldots$ & $\ldots$ & $0.081 \pm 0.013$ & $\ldots$ \\
\hline 135 & $\mathrm{~J} 10122-037$ & AN Sex & 382 & M1.5 V & PMSU & $<2$ & Rein18 & $21.6 \pm 0.2$ & DA19 & $-0.030 \pm 0.012$ & $\ldots$ \\
\hline 136 & $\mathrm{~J} 10125+570$ & LP 092-048 & $\ldots$ & M3.5 V & Lep13 & $<2$ & Rein18 & $\ldots$ & $\ldots$ & $-0.004 \pm 0.015$ & $\ldots$ \\
\hline 137 & J10167-119 & GJ 386 & 386 & M3.0 V & PMSU & $<2$ & Rein18 & $\ldots$ & $\ldots$ & $0.086 \pm 0.011$ & $\ldots$ \\
\hline 138 & $\mathrm{~J} 10196+198$ & AD Leo & 388 & $\mathrm{M} 3.0 \mathrm{~V}+$ & AF15 & $4.35 \pm 0.03$ & HMR14 & $2.2399 \pm 0.0006$ & Mor08 & $-4.53 \pm 0.04$ & $-3.61 \pm 0.01$ \\
\hline 139 & $\mathrm{~J} 10251-102$ & BD-09 3070 & 390 & $\mathrm{M} 1.0 \mathrm{~V}$ & PMSU & $<2$ & Rein18 & $\ldots$ & $\ldots$ & $-0.102 \pm 0.011$ & $\ldots$ \\
\hline 140 & $\mathrm{~J} 10289+008$ & BD+01 2447 & 393 & $\mathrm{M} 2.0 \mathrm{~V}$ & PMSU & $<2$ & Rein18 & $\ldots$ & $\ldots$ & $-0.008 \pm 0.008$ & $\ldots$ \\
\hline 141 & J10350-094 & LP $670-017$ & $\ldots$ & M3.0 V & Sch05 & $<2$ & Rein18 & $\ldots$ & $\ldots$ & $0.037 \pm 0.008$ & 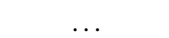 \\
\hline 142 & $\mathrm{~J} 10360+051$ & RY Sex & 398 & M3.5 V & PMSU & $2.9 \pm 1.6$ & Rein18 & $\ldots$ & $\ldots$ & $-5.081 \pm 0.029$ & $-3.64 \pm 0.01$ \\
\hline 143 & $\mathrm{~J} 10396-069$ & GJ 399 & 399 & M2.5 V & PMSU & $<2$ & Rein18 & $\ldots$ & $\ldots$ & $0.002 \pm 0.010$ & $\ldots$ \\
\hline 144 & $\mathrm{~J} 10416+376$ & GJ 1134 & 1134 & M $4.5 \mathrm{~V}$ & PMSU & $<2$ & Rein18 & $54.3 \pm 4.4$ & DA19 & $0.074 \pm 0.015$ & \\
\hline 145 & $\mathrm{~J} 10482-113$ & LP 731-058 & 3622 & M6.5 V & AF15 & $2.1 \pm 1.5$ & Rein18 & $1.5 \pm 0.2$ & Mor10 & $-2.45 \pm 0.05$ & $-4.60 \pm 0.01$ \\
\hline 146 & $\mathrm{~J} 10504+331$ & G 119-037 & 3626 & $\mathrm{M} 4.0 \mathrm{~V}$ & PMSU & $<2$ & Rein18 & & & $0.112 \pm 0.014$ & $\ldots$ \\
\hline 147 & $\mathrm{~J} 10508+068$ & EE Leo & 402 & $\mathrm{M} 4.0 \mathrm{~V}$ & AF15 & $<2$ & Rein18 & $64 \pm 19$ & DA19 & $0.065 \pm 0.011$ & \\
\hline 148 & $\mathrm{~J} 10564+070$ & CN Leo & 406 & M6.0 V & $\mathrm{AF} 15$ & $2.9 \pm 0.8$ & Fou18 & $2.704 \pm 0.003$ & DA19 & $-7.62 \pm 0.07$ & $-3.96 \pm 0.01$ \\
\hline 149 & J10584-107 & LP 731-076 & $\ldots$ & M5.0 V & $\mathrm{AF} 15$ & $3.2 \pm 1.5$ & Rein18 & $\ldots$ & $\ldots$ & $-4.69 \pm 0.04$ & $-3.85 \pm 0.01$ \\
\hline 150 & $\mathrm{~J} 11000+228$ & Ross 104 & 408 & M2.5 V & PMSU & $<2$ & Rein18 & $\ldots$ & $\ldots$ & $-0.032 \pm 0.006$ & \\
\hline 151 & $\mathrm{~J} 11026+219$ & DS Leo & 410 & M1.0 V & Lep13 & $2.6 \pm 1.5$ & Rein18 & $14.6 \pm 0.2$ & DA19 & $-0.489 \pm 0.014$ & $-4.53 \pm 0.02$ \\
\hline 152 & $\mathrm{~J} 11033+359$ & Lalande 21185 & 411 & M1.5 V & AF15 & $<2$ & Rein18 & 48 & KS07 & $-0.035 \pm 0.011$ & $\ldots$ \\
\hline 153 & $\mathrm{~J} 11054+435$ & $\mathrm{BD}+442051 \mathrm{~A}$ & $412 \mathrm{~A}$ & $\mathrm{M} 1.0 \mathrm{~V}$ & AF15 & $<2$ & Rein18 & $100.9 \pm 0.3$ & SM18 & $-0.094 \pm 0.009$ & \\
\hline 154 & $\mathrm{~J} 11055+435$ & WX UMa & $412 \mathrm{~B}$ & M5.5 V & AF15 & $8.2 \pm 2.7$ & Rein18 & $0.78 \pm 0.02$ & Mor10 & $-10.51 \pm 0.16$ & $-3.56 \pm 0.01$ \\
\hline 155 & $\mathrm{~J} 11110+304 \mathrm{~W}$ & HD 97101 B & $414 \mathrm{~B}$ & M2.0 V & Lep13 & $<2$ & Rein18 & $\ldots$ & $\ldots$ & $0.056 \pm 0.015$ & $\ldots$ \\
\hline 156 & $\mathrm{~J} 11126+189$ & StKM 1-928 & 3649 & M1.5 V & PMSU & $<2$ & Rein18 & $\ldots$ & $\ldots$ & $0.088 \pm 0.012$ & $\ldots$ \\
\hline 157 & $\mathrm{~J} 11201-104$ & LP 733-099 & $\ldots$ & $\mathrm{M} 2.0 \mathrm{~V}$ & Ria06 & $3.6 \pm 1.5$ & Rein18 & $\ldots$ & $\ldots$ & $-1.937 \pm 0.016$ & $-3.94 \pm 0.01$ \\
\hline
\end{tabular}


Table A.1.: Identifications, basic parameters, $\mathrm{pEW}_{\mathrm{H}}^{\prime}$ values, and normalised $\mathrm{H} \alpha$ luminosities of the analysed stars (cont.)

\begin{tabular}{|c|c|c|c|c|c|c|c|c|c|c|c|}
\hline No. & Karmn & Name & $\mathrm{Gl} / \mathrm{GJ}$ & SpT & Ref. & $\begin{array}{c}v \sin i \\
{\left[\mathrm{~km} \mathrm{~s}^{-1}\right]}\end{array}$ & Ref. & $\begin{array}{l}P_{\text {rot }} \\
{[\mathrm{d}]}\end{array}$ & Ref. & $\begin{array}{c}\mathrm{pEW}_{\mathrm{H} \alpha}^{\prime} \\
{[\AA]}\end{array}$ & $\log \left(L_{\mathrm{H} \alpha} / L_{\mathrm{bol}}\right)$ \\
\hline 158 & $\mathrm{~J} 11289+101$ & Wolf 398 & 3666 & M3.5 V & PMSU & $<2$ & Rein18 & & & $0.016 \pm 0.013$ & $\ldots$ \\
\hline 159 & $\mathrm{~J} 11302+076$ & K2-18 & $\ldots$ & $\mathrm{M} 2.5 \mathrm{~V}$ & Benn17 & $<2$ & Rein18 & $36.4 \pm 0.3$ & DA19 & $0.026 \pm 0.011$ & $\ldots$ \\
\hline 160 & $\mathrm{~J} 11306-080$ & LP $672-042$ & 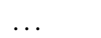 & M3.5 V & $\mathrm{AF} 15$ & $<2$ & Rein18 & & & $0.011 \pm 0.014$ & $\ldots$ \\
\hline 161 & $\mathrm{~J} 11417+427$ & Ross 1003 & 1148 & $\mathrm{M} 4.0 \mathrm{~V}$ & PMSU & $<2$ & Rein 18 & $71.5 \pm 5.1$ & DA19 & $0.11 \pm 0.01$ & $\ldots$ \\
\hline 162 & $\mathrm{~J} 11421+267$ & Ross 905 & 436 & $\mathrm{M} 2.5 \mathrm{~V}$ & $\mathrm{AF} 15$ & $<2$ & Rein 18 & $44.6 \pm 2$ & DA19 & $-0.018 \pm 0.011$ & $\ldots$ \\
\hline 163 & $\mathrm{~J} 11467-140$ & GJ 443 & 443 & M3.0 V & PMSU & $<2$ & Rein18 & $\ldots$ & $\ldots$ & $0.086 \pm 0.010$ & 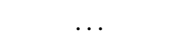 \\
\hline 164 & $\mathrm{~J} 11474+667$ & 1RXS J114728.8+664405 & $\ldots$ & $\mathrm{M} 5.0 \mathrm{~V}$ & $\mathrm{AF} 15$ & $2.7 \pm 1.5$ & Rein 18 & $\ldots$ & $\ldots$ & $-6.86 \pm 0.05$ & $-3.69 \pm 0.01$ \\
\hline 165 & $\mathrm{~J} 11476+002$ & LP 613-049 A & $3685 \mathrm{~A}$ & $\mathrm{M} 4.0 \mathrm{~V}$ & PMSU & $2.4 \pm 1.5$ & Rein 18 & $11.6 \pm 0.04$ & DA19 & $-5.072 \pm 0.029$ & $-3.68 \pm 0.01$ \\
\hline 166 & $\mathrm{~J} 11476+786$ & GJ 445 & 445 & M3.5 V & PMSU & $<2$ & Rein18 & & & $0.003 \pm 0.009$ & $\ldots$ \\
\hline 167 & $\mathrm{~J} 11477+008$ & FI Vir & 447 & $\mathrm{M} 4.0 \mathrm{~V}$ & PMSU & $2.1 \pm 1.0$ & Fou18 & $163 \pm 3$ & DA19 & 0 & $\ldots$ \\
\hline 168 & $\mathrm{~J} 11509+483$ & GJ 1151 & 1151 & $\mathrm{M} 4.5 \mathrm{~V}$ & PMSU & $2.5 \pm 1.0$ & Fou18 & $125 \pm 23$ & DA19 & $-0.102 \pm 0.024$ & $\ldots$ \\
\hline 169 & $\mathrm{~J} 11511+352$ & BD+36 2219 & 450 & M1.5 V & AF15 & $<2$ & Rein 18 & $22.8 \pm 1$ & DA19 & $-0.074 \pm 0.010$ & $\ldots$ \\
\hline 170 & $\mathrm{~J} 12054+695$ & Ross 689 & 3704 & $\mathrm{M} 4.0 \mathrm{~V}$ & PMSU & $<2$ & Rein18 & $100 \pm 10$ & DA19 & $0.018 \pm 0.011$ & $\ldots$ \\
\hline 171 & $\mathrm{~J} 12100-150$ & LP 734-032 & 3707 & M3.5 V & PMSU & $<2$ & Rein18 & $\ldots$ & $\ldots$ & $-0.026 \pm 0.019$ & $\ldots$ \\
\hline 172 & J12111-199 & LTT 4562 & $3708 \mathrm{~A}$ & M3.0 V & PMSU & $<2$ & Rein18 & $\ldots$ & $\ldots$ & $0.024 \pm 0.006$ & $\ldots$ \\
\hline 173 & $\mathrm{~J} 12123+544 \mathrm{~S}$ & HD 238090 & $458 \mathrm{~A}$ & $\mathrm{M} 0.0 \mathrm{~V}$ & PMSU & $<2$ & Rein18 & $\ldots$ & $\ldots$ & $0.03 \pm 0.01$ & $\ldots$ \\
\hline 174 & $\mathrm{~J} 12156+526$ & StKM 2-809 & & $\mathrm{M} 4.0 \mathrm{~V}$ & Lep13 & $35.3 \pm 3.5$ & Rein18 & $\cdots$ & & $-5.56 \pm 0.07$ & $-3.64 \pm 0.01$ \\
\hline 175 & $\mathrm{~J} 12189+111$ & GL Vir & 1156 & $\mathrm{M} 5.0 \mathrm{~V}$ & PMSU & $15.5 \pm 1.6$ & Rein18 & $0.49102 \pm 0.00003$ & DA19 & $-5.82 \pm 0.05$ & $-3.76 \pm 0.01$ \\
\hline 176 & $\mathrm{~J} 12230+640$ & Ross 690 & 463 & $\mathrm{M} 3.0 \mathrm{~V}$ & PMSU & $<2$ & Rein 18 & $32.9 \pm 1.1$ & DA19 & $0.133 \pm 0.026$ & $\ldots$ \\
\hline 177 & $\mathrm{~J} 12248-182$ & Ross 695 & 465 & $\mathrm{M} 2.0 \mathrm{~V}$ & PMSU & $<2$ & Rein18 & $\ldots$ & $\ldots$ & $-0.010 \pm 0.013$ & $\ldots$ \\
\hline 178 & $\mathrm{~J} 12312+086$ & $\mathrm{BD}+092636$ & 471 & $\mathrm{M} 0.5 \mathrm{~V}$ & Lep13 & $<2$ & Rein 18 & $\ldots$ & $\ldots$ & $0.014 \pm 0.008$ & $\ldots$ \\
\hline 179 & $\mathrm{~J} 12350+098$ & GJ 476 & 476 & $\mathrm{M} 2.5 \mathrm{~V}$ & PMSU & $<2$ & Rein 18 & $36.6 \pm 1.3$ & DA19 & $0.099 \pm 0.012$ & $\ldots$ \\
\hline 180 & $\mathrm{~J} 12373-208$ & LP 795-038 & $\ldots$ & $\mathrm{M} 4.0 \mathrm{~V}$ & Sch05 & $<2$ & Rein18 & $\ldots$ & $\ldots$ & $0.089 \pm 0.013$ & $\ldots$ \\
\hline 181 & $\mathrm{~J} 12388+116$ & Wolf 433 & 480 & $\mathrm{M} 3.0 \mathrm{~V}$ & PMSU & $<2$ & Rein18 & $\ldots$ & $\ldots$ & $0.006 \pm 0.020$ & $\ldots$ \\
\hline 182 & $\mathrm{~J} 12428+418$ & G $123-055$ & $\ldots$ & $\mathrm{M} 4.0 \mathrm{~V}$ & Lep13 & $<2$ & Rein18 & $16.2 \pm 0.1$ & DA19 & $-2.416 \pm 0.023$ & $-4.00 \pm 0.01$ \\
\hline 183 & $\mathrm{~J} 12479+097$ & Wolf 437 & 486 & M3.5 V & PMSU & $<2$ & Rein 18 & & & $-0.006 \pm 0.014$ & \\
\hline 184 & $\mathrm{~J} 13005+056$ & FN Vir & 493.1 & $\mathrm{M} 4.5 \mathrm{~V}$ & PMSU & $16.4 \pm 1.6$ & Rein18 & $0.60022 \pm 0.00004$ & DA19 & $-5.13 \pm 0.03$ & $-3.76 \pm 0.01$ \\
\hline 185 & $\mathrm{~J} 13102+477$ & G $177-025$ & $\ldots$ & $\mathrm{M} 5.0 \mathrm{~V}$ & $\mathrm{AF} 15$ & $4.5 \pm 0.9$ & Fou18 & $28.8 \pm 0.8$ & DA19 & $-3.17 \pm 0.06$ & $-4.02 \pm 0.01$ \\
\hline 186 & $\mathrm{~J} 13196+333$ & Ross 1007 & 507.1 & M1.5 V & PMSU & $<2$ & Rein 18 & $48 \pm 4.8$ & SM18 & $0.141 \pm 0.016$ & $\ldots$ \\
\hline 187 & $\mathrm{~J} 13209+342$ & BD+35 2439 & 508.2 & $\mathrm{M} 1.0 \mathrm{~V}$ & PMSU & $<2$ & Rein18 & $\ldots$ & $\ldots$ & $-0.006 \pm 0.011$ & $\ldots$ \\
\hline 188 & $\mathrm{~J} 13229+244$ & Ross 1020 & 3779 & $\mathrm{M} 4.0 \mathrm{~V}$ & PMSU & $<2$ & Rein 18 & $\ldots$ & $\ldots$ & $0.061 \pm 0.012$ & $\ldots$ \\
\hline 189 & $\mathrm{~J} 13283-023 \mathrm{~W}$ & Ross 486A & $512 \mathrm{~A}$ & M $3.0 \mathrm{~V}$ & PMSU & $<2$ & Rein 18 & $\ldots$ & $\ldots$ & $0.059 \pm 0.012$ & $\ldots$ \\
\hline 190 & $\mathrm{~J} 13293+114$ & GJ 513 & 513 & M3.5 V & PMSU & $<2$ & Rein 18 & $\ldots$ & $\ldots$ & $0.052 \pm 0.016$ & $\ldots$ \\
\hline 191 & $\mathrm{~J} 13299+102$ & BD+11 2576 & 514 & M $0.5 \mathrm{~V}$ & PMSU & $2.0 \pm 0.8$ & Fou18 & $30 \pm 0.9$ & SM17 & $-0.010 \pm 0.014$ & $\ldots$ \\
\hline 192 & $\mathrm{~J} 13427+332$ & Ross1015 & 3801 & M3.5 V & Lep13 & $<2$ & Rein 18 & $\ldots$ & $\ldots$ & $-0.022 \pm 0.01$ & $\ldots$ \\
\hline 193 & $\mathrm{~J} 13450+176$ & BD+18 2776 & 525 & $\mathrm{M} 0.0 \mathrm{~V}$ & Lep13 & $2.3 \pm 1.5$ & Rein 18 & .. & & $-0.044 \pm 0.024$ & $\ldots$ \\
\hline 194 & $\mathrm{~J} 13457+148$ & HD 119850 & 526 & $\mathrm{M} 1.5 \mathrm{~V}$ & PMSU & $<2$ & Rein18 & $52.3 \pm 1.7$ & SM15 & $0.135 \pm 0.009$ & $\ldots$ \\
\hline 195 & $\mathrm{~J} 13458-179$ & LP 798-034 & 3804 & M3.5 V & PMSU & $<2$ & Rein 18 & & & $0.010 \pm 0.013$ & $\ldots$ \\
\hline 196 & $\mathrm{~J} 13536+776$ & RX J1353.6+7737 & $\ldots$ & $\mathrm{M} 4.0 \mathrm{~V}$ & Lep13 & $8.9 \pm 1.5$ & Rein18 & $1.23 \pm 0.01$ & DA19 & $-4.012 \pm 0.027$ & $-3.78 \pm 0.01$ \\
\hline 197 & $\mathrm{~J} 13582+125$ & Ross 837 & 3817 & $\mathrm{M} 3.0 \mathrm{~V}$ & PMSU & $<2$ & Rein 18 & $\ldots$ & $\ldots$ & $-0.036 \pm 0.010$ & $\ldots$ \\
\hline
\end{tabular}


Table A.1.: Identifications, basic parameters, $\mathrm{pEW}_{\mathrm{H}_{\alpha}}^{\prime}$ values, and normalised $\mathrm{H} \alpha$ luminosities of the analysed stars (cont.)

\begin{tabular}{|c|c|c|c|c|c|c|c|c|c|c|c|}
\hline No. & Karmn & Name & $\mathrm{Gl} / \mathrm{GJ}$ & $\mathrm{SpT}$ & Ref. & $\begin{array}{c}v \sin i \\
{\left[\mathrm{~km} \mathrm{~s}^{-1}\right]}\end{array}$ & Ref. & $\begin{array}{l}P_{\text {rot }} \\
\text { [d] }\end{array}$ & Ref. & $\begin{array}{c}\mathrm{pEW}_{\mathrm{H} \alpha}^{\prime} \\
{[\AA \AA]}\end{array}$ & $\log \left(L_{\mathrm{H} \alpha} / L_{\mathrm{bol}}\right)$ \\
\hline 198 & J13591-198 & LP 799-007 & 3820 & $\mathrm{M} 4.0 \mathrm{~V}$ & PMSU & $3.2 \pm 1.5$ & Rein18 & $\cdots$ & & $-2.981 \pm 0.013$ & $-3.91 \pm 0.01$ \\
\hline 199 & $\mathrm{~J} 14010-026$ & HD 122303 & 536 & M1.0 V & PMSU & $<2$ & Rein18 & $43.9 \pm 0.8$ & SM17 & $0.006 \pm 0.01$ & $\ldots$ \\
\hline 200 & $\mathrm{~J} 14082+805$ & $\mathrm{BD}+81465$ & 540 & M1.0 V & PMSU & $<2$ & Rein18 & $\ldots$ & & $0.040 \pm 0.013$ & $\ldots$ \\
\hline 201 & $\mathrm{~J} 14152+450$ & Ross 992 & 3836 & $\mathrm{M} 3.0 \mathrm{~V}$ & PMSU & $<2$ & Rein18 & $52.8 \pm 1.4$ & DA19 & $0.056 \pm 0.016$ & \\
\hline 202 & $\mathrm{~J} 14173+454$ & RX J1417.3+4525 & $\ldots$ & M $5.0 \mathrm{~V}$ & Gig10 & $15.9 \pm 1.6$ & Rein18 & $\ldots$ & $\ldots$ & $-3.86 \pm 0.05$ & $-3.94 \pm 0.01$ \\
\hline 203 & $\mathrm{~J} 14251+518$ & tet Boo B & $549 \mathrm{~B}$ & $\mathrm{M} 2.5 \mathrm{~V}$ & AF15 & $<2$ & Rein18 & $\ldots$ & $\ldots$ & $-0.011 \pm 0.008$ & $\ldots$ \\
\hline 204 & $\mathrm{~J} 14257+236 \mathrm{E}$ & $\mathrm{BD}+24$ 2733B & $548 \mathrm{~B}$ & $\mathrm{M} 0.5 \mathrm{~V}$ & PMSU & $<2$ & Rein18 & $17.6 \pm 0.5$ & DA19 & $0.017 \pm 0.008$ & $\ldots$ \\
\hline 205 & $\mathrm{~J} 14257+236 \mathrm{~W}$ & $\mathrm{BD}+242733 \mathrm{~A}$ & $548 \mathrm{~A}$ & $\mathrm{M} 0.0 \mathrm{~V}$ & PMSU & $<2$ & Rein18 & $111 \pm 12$ & DA19 & 0 & $\ldots$ \\
\hline 206 & $\mathrm{~J} 14294+155$ & Ross 130 & 552 & $\mathrm{M} 2.0 \mathrm{~V}$ & PMSU & $<2$ & Rein18 & $43.5 \pm 1.5$ & SM18 & $0.044 \pm 0.010$ & $\ldots$ \\
\hline 207 & J14307-086 & BD-07 3856 & 553 & $\mathrm{M} 0.5 \mathrm{~V}$ & Gra03 & $2.4 \pm 1.5$ & Rein18 & $\ldots$ & $\ldots$ & $0.176 \pm 0.014$ & $\ldots$ \\
\hline 208 & $\mathrm{~J} 14310-122$ & Wolf 1478 & 553.1 & $\mathrm{M} 3.5 \mathrm{~V}$ & PMSU & $<2$ & Rein18 & $\ldots$ & $\ldots$ & $-0.008 \pm 0.014$ & $\ldots$ \\
\hline 209 & $\mathrm{~J} 14321+081$ & LP 560-035 & & M6.0 V & New14 & $6.3 \pm 1.5$ & Rein18 & $\ldots$ & $\ldots$ & $-4.76 \pm 0.06$ & $-4.16 \pm 0.01$ \\
\hline 210 & J14342-125 & HN Lib & 555 & $\mathrm{M} 4.0 \mathrm{~V}$ & PMSU & $<2$ & Rein18 & $\ldots$ & $\ldots$ & $0.031 \pm 0.011$ & $\ldots$ \\
\hline 211 & $\mathrm{~J} 14524+123$ & G 066-037 & 3871 & $\mathrm{M} 2.0 \mathrm{~V}$ & PMSU & $<2$ & Rein18 & $\ldots$ & $\ldots$ & $-0.051 \pm 0.009$ & $\ldots$ \\
\hline 212 & $\mathrm{~J} 14544+355$ & Ross 1041 & 3873 & M3.5 V & PMSU & $<2$ & Rein18 & $\ldots$ & $\ldots$ & $-0.013 \pm 0.016$ & $\ldots$ \\
\hline 213 & $\mathrm{~J} 15013+055$ & G 015-002 & 3885 & $\mathrm{M} 3.0 \mathrm{~V}$ & PMSU & $<2$ & Rein18 & $\ldots$ & $\ldots$ & $-0.055 \pm 0.009$ & $\ldots$ \\
\hline 214 & $\mathrm{~J} 15095+031$ & Ross 1047 & 3892 & $\mathrm{M} 3.0 \mathrm{~V}$ & PMSU & $<2$ & Rein18 & $\ldots$ & $\ldots$ & $0.051 \pm 0.010$ & $\ldots$ \\
\hline 215 & J15194-077 & HO Lib & 581 & $\mathrm{M} 3.0 \mathrm{~V}$ & PMSU & $<2$ & Rein18 & $132.5 \pm 6.3$ & SM15 & 0 & $\ldots$ \\
\hline 216 & $\mathrm{~J} 15218+209$ & OT Ser & 9520 & $\mathrm{M} 1.5 \mathrm{~V}$ & PMSU & $4.3 \pm 1.5$ & Rein18 & $3.37 \pm 0.01$ & DA19 & $-2.905 \pm 0.018$ & $-3.76 \pm 0.01$ \\
\hline 217 & $\mathrm{~J} 15305+094$ & NLTT 40406 & & M5.5 V & $\mathrm{AF} 15$ & $16.3 \pm 1.6$ & Rein18 & $0.3048 \pm 0.0006$ & DA19 & $-4.14 \pm 0.05$ & $-3.96 \pm 0.01$ \\
\hline 218 & J15369-141 & Ross 802 & 592 & $\mathrm{M} 4.0 \mathrm{~V}$ & PMSU & $<2$ & Rein18 & $\ldots$ & $\ldots$ & $0.086 \pm 0.013$ & $\ldots$ \\
\hline 219 & $\mathrm{~J} 15474-108$ & LP 743-031 & 3916 & $\mathrm{M} 2.0 \mathrm{~V}$ & PMSU & $<3$ & Jeff 18 & $\ldots$ & $\ldots$ & $-0.038 \pm 0.019$ & . \\
\hline 220 & $\mathrm{~J} 15499+796$ & LP $022-420$ & & M5.0 V & AF15 & $26.9 \pm 2.7$ & Rein18 & & & $-5.16 \pm 0.05$ & $-3.81 \pm 0.01$ \\
\hline 221 & J15598-082 & BD-07 4156 & 606 & M1.0 V & PMSU & $<2$ & Rein18 & $20 \pm 2$ & SM18 & $-0.125 \pm 0.009$ & $\ldots$ \\
\hline 222 & $\mathrm{~J} 16028+205$ & GJ 609 & 609 & $\mathrm{M} 4.0 \mathrm{~V}$ & PMSU & $<2$ & Rein18 & $\ldots$ & $\ldots$ & $0.041 \pm 0.010$ & $\ldots$ \\
\hline 223 & $\mathrm{~J} 16092+093$ & G $137-084$ & $\ldots$ & $\mathrm{M} 3.0 \mathrm{~V}$ & Lep13 & $<2$ & Rein18 & $\ldots$ & & $-0.051 \pm 0.009$ & \\
\hline 224 & J16102-193 & K2-33 & $\ldots$ & $\mathrm{M} 3.0 \mathrm{~V}$ & Davi16 & $7.3 \pm 1.5$ & Rein18 & $6.263 \pm 0.150$ & DA19 & $-1.48 \pm 0.04$ & $-4.10 \pm 0.02$ \\
\hline 225 & $\mathrm{~J} 16167+672 \mathrm{~N}$ & EW Dra & $617 \mathrm{~B}$ & $\mathrm{M} 3.0 \mathrm{~V}$ & PMSU & $<2$ & Rein18 & $\ldots$ & $\ldots$ & $0.062 \pm 0.010$ & $\ldots$ \\
\hline 226 & $\mathrm{~J} 16167+672 \mathrm{~S}$ & HD 147379 & $617 \mathrm{~A}$ & $\mathrm{M} 0.0 \mathrm{~V}$ & $\mathrm{AF} 15$ & $2.7 \pm 1.5$ & Rein18 & $\ldots$ & $\ldots$ & $0.052 \pm 0.012$ & $\ldots$ \\
\hline 227 & $\mathrm{~J} 16254+543$ & GJ 625 & 625 & M1.5 V & AF15 & $2.2 \pm 0.7$ & Fou18 & $76.79 \pm 0.13$ & DA19 & 0 & $\ldots$ \\
\hline 228 & J16303-126 & V2306 Oph & 628 & M $3.5 \mathrm{~V}$ & PMSU & $<2$ & Rein18 & $119 \pm 1$ & DA19 & $0.011 \pm 0.012$ & $\ldots$ \\
\hline 229 & $\mathrm{~J} 16313+408$ & G $180-060$ & 3959 & $\mathrm{M} 5.0 \mathrm{~V}$ & PMSU & $7.1 \pm 1.5$ & Rein18 & $0.512 \pm 0.001$ & DA19 & $-7.44 \pm 0.04$ & $-3.65 \pm 0.01$ \\
\hline 230 & $\mathrm{~J} 16327+126$ & GJ 1203 & 1203 & $\mathrm{M} 3.0 \mathrm{~V}$ & PMSU & $<2$ & Rein18 & $\ldots$ & $\ldots$ & $0.117 \pm 0.014$ & $\ldots$ \\
\hline 231 & $\mathrm{~J} 16462+164$ & LP 446-006 & 3972 & $\mathrm{M} 2.5 \mathrm{~V}$ & PMSU & $<2$ & Rein18 & $\ldots$ & $\ldots$ & $-0.001 \pm 0.008$ & $\ldots$ \\
\hline 232 & $\mathrm{~J} 16554-083 \mathrm{~N}$ & GJ 643 & 643 & $\mathrm{M} 3.5 \mathrm{~V}$ & PMSU & $<2$ & Rein18 & $6.52 \pm 0.01$ & DA19 & $-0.001 \pm 0.010$ & $\cdots$ \\
\hline 233 & J16555-083 & vB 8 & $644 \mathrm{C}$ & $\mathrm{M} 7.0 \mathrm{~V}$ & AF15 & $10.1 \pm 0.8$ & Fou18 & $\ldots$ & $\ldots$ & $-4.87 \pm 0.11$ & $-4.37 \pm 0.01$ \\
\hline 234 & $\mathrm{~J} 16570-043$ & LP 686-027 & 1207 & M3.5 V & PMSU & $10.1 \pm 1.5$ & Rein18 & $0.547 \pm 0.001$ & DA19 & $-4.563 \pm 0.015$ & $-3.69 \pm 0.01$ \\
\hline 235 & $\mathrm{~J} 16581+257$ & $\mathrm{BD}+253173$ & 649 & M1.0 V & PMSU & $<2$ & Rein18 & $23.8 \pm 0.1$ & DA19 & $-0.049 \pm 0.006$ & $\ldots$ \\
\hline 236 & $\mathrm{~J} 17033+514$ & G 203-042 & 3988 & $\mathrm{M} 4.5 \mathrm{~V}$ & PMSU & $<2$ & Rein18 & $\ldots$ & $\ldots$ & $-0.003 \pm 0.013$ & $\ldots$ \\
\hline 237 & $\mathrm{~J} 17052-050$ & Wolf 636 & 654 & $\mathrm{M} 1.5 \mathrm{~V}$ & AF15 & $<2$ & Rein18 & $50.2 \pm 1.3$ & DA19 & $0.103 \pm 0.016$ & $\ldots$ \\
\hline
\end{tabular}


Table A.1.: Identifications, basic parameters, $\mathrm{pEW}_{\mathrm{H}}^{\prime}$ values, and normalised $\mathrm{H} \alpha$ luminosities of the analysed stars (cont.)

\begin{tabular}{|c|c|c|c|c|c|c|c|c|c|c|c|}
\hline No. & Karmn & Name & $\mathrm{Gl} / \mathrm{GJ}$ & $\mathrm{SpT}$ & Ref. & $\begin{array}{c}v \sin i \\
{\left[\mathrm{~km} \mathrm{~s}^{-1}\right]}\end{array}$ & Ref. & $\begin{array}{l}P_{\text {rot }} \\
\text { [d] }\end{array}$ & Ref. & $\begin{array}{c}\mathrm{pEW}_{\mathrm{H} \alpha}^{\prime} \\
{[\AA]}\end{array}$ & $\log \left(L_{\mathrm{H} \alpha} / L_{\mathrm{bol}}\right)$ \\
\hline 238 & $\mathrm{~J} 17071+215$ & Ross 863 & 655 & M3.0 V & PMSU & $<2$ & Rein 18 & & & $0.064 \pm 0.008$ & $\ldots$ \\
\hline 239 & $\mathrm{~J} 17115+384$ & Wolf 654 & 3992 & M3.5 V & PMSU & $<2$ & Rein 18 & $62.6 \pm 2$ & DA19 & $0.082 \pm 0.014$ & $\ldots$ \\
\hline 240 & $\mathrm{~J} 17166+080$ & GJ 2128 & 2128 & $\mathrm{M} 2.0 \mathrm{~V}$ & PMSU & $<2$ & Rein 18 & & & $-0.004 \pm 0.007$ & $\ldots$ \\
\hline 241 & $J 17198+417$ & GJ 671 & 671 & $\mathrm{M} 2.5 \mathrm{~V}$ & PMSU & $<2$ & Rein18 & $71.5 \pm 2.6$ & DA19 & 0 & $\ldots$ \\
\hline 242 & $\mathrm{~J} 17303+055$ & BD+05 3409 & $678.1 \mathrm{~A}$ & $\mathrm{M} 0.0 \mathrm{~V}$ & PMSU & $<2$ & Rein18 & & & $-0.038 \pm 0.013$ & \\
\hline 243 & $\mathrm{~J} 17338+169$ & 1RXS J173353.5+165515 & $\ldots$ & M5.5 V & Lep13 & $41.5 \pm 7.7$ & Rein 18 & $0.26593 \pm 0.0003$ & DA19 & $-12.17 \pm 0.07$ & $-3.49 \pm 0.01$ \\
\hline 244 & $\mathrm{~J} 17355+616$ & $\mathrm{BD}+611678 \mathrm{C}$ & 685 & $\mathrm{M} 0.5 \mathrm{~V}$ & PMSU & $<2$ & Rein 18 & $19.3 \pm 0.3$ & DA19 & $-0.088 \pm 0.013$ & $\ldots$ \\
\hline 245 & $\mathrm{~J} 17364+683$ & $\mathrm{BD}+68946 \mathrm{AB}$ & $687 \mathrm{AB}$ & $\mathrm{M} 3.0 \mathrm{~V}+$ & AF15 & $<2.5$ & Bro10 & $\ldots$ & $\ldots$ & $0.023 \pm 0.026$ & $\ldots$ \\
\hline 246 & $\mathrm{~J} 17378+185$ & $\mathrm{BD}+183421$ & 686 & $\mathrm{M} 1.0 \mathrm{~V}$ & PMSU & $<2$ & Rein 18 & $\ldots$ & $\ldots$ & $-0.058 \pm 0.006$ & $\ldots$ \\
\hline 247 & $\mathrm{~J} 17542+073$ & GJ 1222 & 1222 & $\mathrm{M} 4.0 \mathrm{~V}$ & PMSU & $<2$ & Rein 18 & & 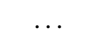 & $0.09 \pm 0.04$ & $\ldots$ \\
\hline 248 & $\mathrm{~J} 17578+046$ & Barnard's Star & 699 & M3.5 V & AF15 & $3.1 \pm 1.2$ & Fou18 & $148.6 \pm 0.1$ & SM15 & 0 & $\ldots$ \\
\hline 249 & $\mathrm{~J} 17578+465$ & G 204-039 & 4040 & $\mathrm{M} 2.5 \mathrm{~V}$ & AF15 & $2 \pm 1.1$ & Fou18 & $30.3 \pm 0.9$ & DA19 & $-0.187 \pm 0.024$ & \\
\hline 250 & $\mathrm{~J} 18022+642$ & LP 071-082 & $\ldots$ & M5.0 V & AF15 & $11.3 \pm 1.5$ & Rein 18 & $0.28027 \pm 0.00002$ & DA19 & $-5.17 \pm 0.06$ & $-3.81 \pm 0.01$ \\
\hline 251 & $\mathrm{~J} 18027+375$ & GJ 1223 & 1223 & M5.0 V & PMSU & $<2$ & Rein18 & 123.8 & New16 & $0.050 \pm 0.014$ & $\ldots$ \\
\hline 252 & $\mathrm{~J} 18051-030$ & HD 165222 & 701 & M1.0 V & PMSU & $<2$ & Rein 18 & $127.8 \pm 3.2$ & SM15 & 0 & 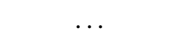 \\
\hline 253 & J18075-159 & GJ 1224 & 1224 & $\mathrm{M} 4.5 \mathrm{~V}$ & PMSU & $2.2 \pm 1.5$ & Rein 18 & $3.866 \pm 0.008$ & DA19 & $-4.65 \pm 0.04$ & $-3.81 \pm 0.01$ \\
\hline 254 & $\mathrm{~J} 18131+260$ & LP 390-016 & 4044 & $\mathrm{M} 4.0 \mathrm{~V}$ & AF15 & $7.5 \pm 0.7$ & Fou18 & $2.28 \pm 0.01$ & DA19 & $-6.021 \pm 0.027$ & $-3.60 \pm 0.01$ \\
\hline 255 & $\mathrm{~J} 18165+048$ & G $140-051$ & $\ldots$ & M5.0 V & New14 & $<2$ & Rein 18 & $\ldots$ & $\ldots$ & $0.103 \pm 0.013$ & $\ldots$ \\
\hline 256 & $\mathrm{~J} 18174+483$ & TYC 3529-1437-1 & $\ldots$ & $\mathrm{M} 2.0 \mathrm{~V}$ & Ria06 & $3.1 \pm 1$ & Fou18 & $15.8 \pm 0.1$ & DA19 & $-1.697 \pm 0.015$ & $-4.00 \pm 0.01$ \\
\hline 257 & $\mathrm{~J} 18180+387 \mathrm{E}$ & G 204-058 & $4048 \mathrm{~A}$ & M3.0 V & PMSU & $<2$ & Rein 18 & $\ldots$ & $\ldots$ & $0.034 \pm 0.007$ & $\ldots$ \\
\hline 258 & J18189+661 & LP 071-165 & 4053 & $\mathrm{M} 4.5 \mathrm{~V}$ & PMSU & $15.3 \pm 1.5$ & Rein18 & $\ldots$ & $\ldots$ & $-2.58 \pm 0.05$ & $-4.06 \pm 0.01$ \\
\hline 259 & $\mathrm{~J} 18221+063$ & Ross 136 & 712 & $\mathrm{M} 4.0 \mathrm{~V}$ & PMSU & $<2$ & Rein 18 & $\ldots$ & $\ldots$ & $0.148 \pm 0.019$ & $\ldots$ \\
\hline 260 & $\mathrm{~J} 18224+620$ & GJ 1227 & 1227 & $\mathrm{M} 4.0 \mathrm{~V}$ & AF15 & $<2$ & Rein 18 & $\ldots$ & $\ldots$ & $-0.088 \pm 0.022$ & $\ldots$ \\
\hline 261 & $\mathrm{~J} 18319+406$ & G $205-028$ & 4062 & M3.5 V & PMSU & $<2$ & Rein 18 & $50.2 \pm 1.3$ & DA19 & $-0.032 \pm 0.017$ & $\ldots$ \\
\hline 262 & $\mathrm{~J} 18346+401$ & LP 229-017 & 4063 & M3.5 V & PMSU & $<2$ & Rein18 & $40.2 \pm 0.8$ & DA19 & $-0.039 \pm 0.016$ & $\ldots$ \\
\hline 263 & $\mathrm{~J} 18353+457$ & $\mathrm{BD}+452743$ & $720 \mathrm{~A}$ & M0.5 V & AF15 & $<2$ & Rein 18 & $34 \pm 2.9$ & DA19 & $0.109 \pm 0.006$ & \\
\hline 264 & $\mathrm{~J} 18356+329$ & LSR J1835+3259 & $\ldots$ & M8.5 V & Schm07 & $49.2 \pm 4.9$ & Rein 18 & $0.118 \pm 0.001$ & DA19 & $-1.92 \pm 0.16$ & $-4.99 \pm 0.04$ \\
\hline 265 & $\mathrm{~J} 18363+136$ & Ross 149 & 4065 & $\mathrm{M} 4.0 \mathrm{~V}$ & PMSU & $<2$ & Rein18 & $50.2 \pm 1.6$ & DA19 & $-0.735 \pm 0.015$ & $-4.52 \pm 0.01$ \\
\hline 266 & $\mathrm{~J} 18409-133$ & BD-13 5069 & 724 & M1.0 V & PMSU & $<2$ & Rein 18 & $\ldots$ & $\ldots$ & $0.03 \pm 0.01$ & $\ldots$ \\
\hline 267 & $\mathrm{~J} 18419+318$ & Ross 145 & 4070 & $\mathrm{M} 3.0 \mathrm{~V}$ & PMSU & $<2$ & Rein 18 & $\ldots$ & $\ldots$ & $0.042 \pm 0.007$ & $\ldots$ \\
\hline 268 & $\mathrm{~J} 18427+596 \mathrm{~N}$ & HD 173739 & $725 \mathrm{~A}$ & M3.0 V & $\mathrm{AF} 15$ & $<2.5$ & Bro10 & $\ldots$ & $\ldots$ & $0.004 \pm 0.019$ & $\ldots$ \\
\hline 269 & $\mathrm{~J} 18427+596 \mathrm{~S}$ & HD 173740 & $725 \mathrm{~B}$ & M3.5 V & AF15 & $<2.5$ & Bro10 & $\ldots$ & $\ldots$ & $0.014 \pm 0.021$ & $\ldots$ \\
\hline 270 & $\mathrm{~J} 18480-145$ & G $155-042$ & 4077 & M2.5 V & PMSU & $<2$ & Rein18 & $\ldots$ & $\ldots$ & $-0.007 \pm 0.007$ & $\ldots$ \\
\hline 271 & $\mathrm{~J} 18482+076$ & G 141-036 & $\ldots$ & M5.0 V & AF15 & $2.4 \pm 1.5$ & Rein 18 & $2.76 \pm 0.01$ & DA19 & $-3.217 \pm 0.019$ & $-4.02 \pm 0.01$ \\
\hline 272 & $\mathrm{~J} 18498-238$ & V1216 Sgr & 729 & $\mathrm{M} 3.5 \mathrm{~V}$ & Ria06 & $3 \pm 1.5$ & Rein18 & $2.87 \pm 0.01$ & DA19 & $-2.258 \pm 0.019$ & $-3.99 \pm 0.01$ \\
\hline 273 & $\mathrm{~J} 18580+059$ & $\mathrm{BD}+053993$ & 740 & M0.5 V & PMSU & $<2$ & Rein 18 & $35.2 \pm 0.3$ & DA19 & 0 & $\ldots$ \\
\hline 274 & $\mathrm{~J} 19070+208$ & Ross 730 & $745 \mathrm{~A}$ & $\mathrm{M} 2.0 \mathrm{~V}$ & AF15 & $<2$ & Rein 18 & $\ldots$ & $\ldots$ & $-0.116 \pm 0.017$ & $\ldots$ \\
\hline 275 & $\mathrm{~J} 19072+208$ & HD 349726 & $745 \mathrm{~B}$ & M2.0 V & PMSU & $<2$ & Rein18 & $3.8 \pm 0.01$ & DA19 & $-0.104 \pm 0.016$ & $\ldots$ \\
\hline 276 & $\mathrm{~J} 19084+322$ & G 207-019 & 4098 & M3.0 V & PMSU & $<2$ & Rein18 & $74.1 \pm 2.8$ & DA19 & $0.030 \pm 0.008$ & $\ldots$ \\
\hline 277 & $\mathrm{~J} 19098+176$ & GJ 1232 & 1232 & M4.5 V & PMSU & $<2$ & Rein18 & $80.1 \pm 3.2$ & DA19 & $0.003 \pm 0.013$ & $\ldots$ \\
\hline
\end{tabular}


Table A.1.: Identifications, basic parameters, $\mathrm{pEW}_{\mathrm{H}^{\prime}}^{\prime}$ values, and normalised $\mathrm{H} \alpha$ luminosities of the analysed stars (cont.)

\begin{tabular}{|c|c|c|c|c|c|c|c|c|c|c|c|}
\hline No. & Karmn & Name & Gl/GJ & $\mathrm{SpT}$ & Ref. & $\begin{array}{c}v \sin i \\
{\left[\mathrm{~km} \mathrm{~s}^{-1}\right]}\end{array}$ & Ref. & $\begin{array}{l}P_{\text {rot }} \\
\text { [d] }\end{array}$ & Ref. & $\begin{array}{c}\mathrm{pEW}_{\mathrm{H} \alpha}^{\prime} \\
[\AA]]\end{array}$ & $\log \left(L_{\mathrm{H} \alpha} / L_{\mathrm{bol}}\right)$ \\
\hline 278 & $\mathrm{~J} 19169+051 \mathrm{~N}$ & V1428 Aql & $752 \mathrm{~A}$ & $\mathrm{M} 2.5 \mathrm{~V}$ & $\mathrm{AF} 15$ & $<2$ & Rein18 & $46 \pm 0.2$ & DA19 & $0.028 \pm 0.009$ & \\
\hline 279 & $\mathrm{~J} 19169+051 \mathrm{~S}$ & V1298 Aql (vB 10) & $752 \mathrm{~B}$ & $\mathrm{M} 8.0 \mathrm{~V}$ & $\mathrm{AF} 15$ & $5.3 \pm 0.9$ & Fou18 & $23.6 \pm 0.3$ & DA19 & $-5.33 \pm 0.09$ & $-4.45 \pm 0.01$ \\
\hline 280 & $\mathrm{~J} 19216+208$ & GJ 1235 & 1235 & $\mathrm{M} 4.5 \mathrm{~V}$ & PMSU & $<2$ & Rein 18 & $133 \pm 9$ & DA19 & 0 & $\ldots$ \\
\hline 281 & $\mathrm{~J} 19251+283$ & Ross 164 & 4109 & $\mathrm{M} 3.0 \mathrm{~V}$ & PMSU & $<2$ & Rein 18 & $\ldots$ & $\ldots$ & $-0.070 \pm 0.019$ & $\ldots$ \\
\hline 282 & $\mathrm{~J} 19255+096$ & LSPM J1925+0938 & & M $8.0 \mathrm{~V}$ & New14 & $34.7 \pm 3.5$ & Rein18 & $\ldots$ & $\cdots$ & $2.07 \pm 0.08$ & $\ldots$ \\
\hline 283 & $\mathrm{~J} 19346+045$ & $\mathrm{BD}+044157$ & 763 & $\mathrm{M} 0.0 \mathrm{~V}$ & Kir91 & $3.9 \pm 1.5$ & Rein 18 & $12.9 \pm 0.8$ & DA19 & $0.087 \pm 0.018$ & \\
\hline 284 & J19422-207 & 2M J19421282-2045477 & & M5.1 V & Shk09 & $6.2 \pm 1.5$ & Rein18 & & & $-5.73 \pm 0.04$ & $-3.77 \pm 0.01$ \\
\hline 285 & $\mathrm{~J} 19511+464$ & G 208-042 & 1243 & $\mathrm{M} 4.0 \mathrm{~V}$ & PMSU & $22.1 \pm 0.9$ & Fou18 & $0.59278 \pm 0.00012$ & DA19 & $-4.49 \pm 0.03$ & $-3.73 \pm 0.01$ \\
\hline 286 & J20093-012 & 2M J20091824-0113377 & .. & M5.0 V & AF15 & $4.3 \pm 1.5$ & Rein 18 & & & $-5.108 \pm 0.026$ & $-3.82 \pm 0.01$ \\
\hline 287 & $\mathrm{~J} 20260+585$ & Wolf 1069 & 1253 & M5.0 V & PMSU & $<2$ & Rein 18 & $57.7 \pm 0.4$ & DA19 & $0.001 \pm 0.009$ & $\ldots$ \\
\hline 288 & $\mathrm{~J} 20305+654$ & GJ 793 & 793 & $\mathrm{M} 2.5 \mathrm{~V}$ & PMSU & $<2$ & Rein 18 & $32.8 \pm 0.5$ & DA19 & $-0.241 \pm 0.010$ & $\ldots$ \\
\hline 289 & $\mathrm{~J} 20336+617$ & GJ 1254 & 1254 & $\mathrm{M} 4.0 \mathrm{~V}$ & PMSU & $<2$ & Rein 18 & $12.6 \pm 0.7$ & DA19 & $0.065 \pm 0.012$ & $\ldots$ \\
\hline 290 & $\mathrm{~J} 20405+154$ & GJ 1256 & 1256 & $\mathrm{M} 4.5 \mathrm{~V}$ & AF15 & $<2$ & Rein18 & $106 \pm 6$ & DA19 & $-0.195 \pm 0.012$ & $\ldots$ \\
\hline 291 & $\mathrm{~J} 20450+444$ & $\mathrm{BD}+443567$ & 806 & $\mathrm{M} 1.5 \mathrm{~V}$ & PMSU & $<2$ & Rein18 & $19.9 \pm 0.6$ & DA19 & $0.061 \pm 0.010$ & \\
\hline 292 & J20451-313 & AU Mic & 803 & $\mathrm{M} 0.5 \mathrm{~V}$ & Ria06 & $9.3 \pm 1.2$ & Tor06 & $4.84 \pm 0.04$ & Mes11 & $-3.028 \pm 0.018$ & $-3.71 \pm 0.01$ \\
\hline 293 & J20525-169 & LP 816-060 & $\ldots$ & $\mathrm{M} 4.0 \mathrm{~V}$ & Gra06 & $<2$ & Rein 18 & $67.6 \pm 0.6$ & DA19 & $-0.012 \pm 0.010$ & $\ldots$ \\
\hline 294 & $\mathrm{~J} 20533+621$ & HD 199305 & $809 \mathrm{~A}$ & M1.0 V & Lep13 & $<2$ & Rein 18 & $\ldots$ & $\ldots$ & $-0.010 \pm 0.009$ & $\ldots$ \\
\hline 295 & J20556-140S & GJ $810 \mathrm{~B}$ & $810 \mathrm{~B}$ & M5.0 V & PMSU & $\ldots$ & $\ldots$ & $\ldots$ & $\ldots$ & $0.148 \pm 0.017$ & $\ldots$ \\
\hline 296 & J20567-104 & Wolf 896 & 811.1 & $\mathrm{M} 2.5 \mathrm{~V}$ & PMSU & $<2$ & Rein 18 & $9.63 \pm 0.01$ & DA19 & $0.005 \pm 0.011$ & $\ldots$ \\
\hline 297 & J21019-063 & Wolf 906 & 816 & $\mathrm{M} 2.5 \mathrm{~V}$ & $\mathrm{AF} 15$ & $<2$ & Rein 18 & $\ldots$ & $\ldots$ & $0.012 \pm 0.008$ & $\ldots$ \\
\hline 298 & $\mathrm{~J} 21152+257$ & LP 397-041 & 4184 & $\mathrm{M} 3.0 \mathrm{~V}$ & PMSU & $<2$ & Rein18 & $34.8 \pm 0.2$ & DA19 & $0.100 \pm 0.013$ & $\ldots$ \\
\hline 299 & $\mathrm{~J} 21164+025$ & LSPM J2116+0234 & $\ldots$ & $\mathrm{M} 3.0 \mathrm{~V}$ & Lep13 & $<2$ & Rein 18 & $\ldots$ & $\ldots$ & $0.102 \pm 0.005$ & $\ldots$ \\
\hline 300 & $\mathrm{~J} 21221+229$ & TYC 2187-512-1 & $\ldots$ & M1.0 V & Lep13 & $<2$ & Rein 18 & $41 \pm 1.7$ & DA19 & $-0.027 \pm 0.006$ & $\ldots$ \\
\hline 301 & $\mathrm{~J} 21348+515$ & Wolf 926 & 4205 & $\mathrm{M} 3.0 \mathrm{~V}$ & PMSU & $<2$ & Rein 18 & $54.3 \pm 1.5$ & DA19 & $0.075 \pm 0.013$ & $\ldots$ \\
\hline 302 & $\mathrm{~J} 21463+382$ & LSPM J2146+3813 & $\ldots$ & $\mathrm{M} 4.0 \mathrm{~V}$ & Lep13 & $<2$ & Rein18 & $\ldots$ & $\ldots$ & $0.095 \pm 0.013$ & $\ldots$ \\
\hline 303 & J21466-001 & Wolf 940 & $1263 \mathrm{~A}$ & $\mathrm{M} 4.0 \mathrm{~V}$ & PMSU & $<2$ & Rein 18 & $\ldots$ & $\ldots$ & $0.089 \pm 0.010$ & $\ldots$ \\
\hline 304 & $\mathrm{~J} 21466+668$ & G 264-012 & $\ldots$ & $\mathrm{M} 4.0 \mathrm{~V}$ & $\mathrm{AF} 15$ & $<2$ & Rein 18 & $\ldots$ & $\ldots$ & $0.076 \pm 0.010$ & 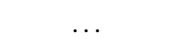 \\
\hline 305 & $\mathrm{~J} 22012+283$ & V374 Peg & 4247 & $\mathrm{M} 4.0 \mathrm{~V}$ & PMSU & $36.9 \pm 0.7$ & Fou 18 & $0.44571 \pm 0.00002$ & DA19 & $-4.49 \pm 0.04$ & $-3.73 \pm 0.01$ \\
\hline 306 & J22020-194 & LP $819-017$ & 843 & $\mathrm{M} 3.5 \mathrm{~V}$ & PMSU & $<2$ & Rein 18 & $\ldots$ & $\ldots$ & $0.064 \pm 0.019$ & $\ldots$ \\
\hline 307 & $\mathrm{~J} 22021+014$ & $\mathrm{BD}+004810$ & 846 & $\mathrm{M} 0.5 \mathrm{~V}$ & AF15 & $<2$ & Rein 18 & $29.5 \pm 0.1$ & DA19 & $0.019 \pm 0.005$ & $\ldots$ \\
\hline 308 & $\mathrm{~J} 22057+656$ & G 264-018 A & 4258 & $\mathrm{M} 1.5 \mathrm{~V}$ & PMSU & $<2$ & Rein 18 & & & $0.111 \pm 0.011$ & $\ldots$ \\
\hline 309 & J22096-046 & BD-05 5715 & 849 & $\mathrm{M} 3.5 \mathrm{~V}$ & PMSU & $<2$ & Rein18 & $39.2 \pm 6.3$ & SM15 & $0.143 \pm 0.019$ & $\ldots$ \\
\hline 310 & $\mathrm{~J} 22114+409$ & 1RXS J221124.3+410000 & & M5.5 V & $\mathrm{AF} 15$ & $<2$ & Rein 18 & $30 \pm 1.3$ & DA19 & $-4.94 \pm 0.04$ & $-3.89 \pm 0.01$ \\
\hline 311 & $\mathrm{~J} 22115+184$ & Ross 271 & 851 & $\mathrm{M} 2.0 \mathrm{~V}$ & PMSU & $<2$ & Rein18 & $36.3 \pm 0.2$ & DA19 & $0.056 \pm 0.010$ & $\ldots$ \\
\hline 312 & $\mathrm{~J} 22125+085$ & Wolf 1014 & 9773 & $\mathrm{M} 3.0 \mathrm{~V}$ & PMSU & $<2$ & Rein18 & $\ldots$ & $\ldots$ & $0.034 \pm 0.006$ & $\ldots$ \\
\hline 313 & J22137-176 & LP $819-052$ & 1265 & $\mathrm{M} 4.5 \mathrm{~V}$ & PMSU & $<2$ & Rein 18 & $\ldots$ & $\ldots$ & $0.042 \pm 0.017$ & 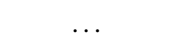 \\
\hline 314 & J22231-176 & LP $820-012$ & 4274 & $\mathrm{M} 4.5 \mathrm{~V}$ & PMSU & $<2$ & Rein 18 & $\ldots$ & $\ldots$ & $-3.977 \pm 0.022$ & $-3.87 \pm 0.01$ \\
\hline 315 & $\mathrm{~J} 22252+594$ & G 232-070 & 4276 & $\mathrm{M} 4.0 \mathrm{~V}$ & PMSU & $<2$ & Rein 18 & $64.6 \pm 2.1$ & DA19 & $0.077 \pm 0.010$ & $\ldots$ \\
\hline 316 & $\mathrm{~J} 22298+414$ & G 215-050 & 1270 & $\mathrm{M} 4.0 \mathrm{~V}$ & PMSU & $<2$ & Rein 18 & $99.8 \pm 1.2$ & DA19 & $0.076 \pm 0.010$ & $\ldots$ \\
\hline 317 & $\mathrm{~J} 22330+093$ & BD+08 4887 & 863 & M1.0 V & PMSU & $<2$ & Rein 18 & $\ldots$ & $\ldots$ & $-0.014 \pm 0.005$ & $\ldots$ \\
\hline
\end{tabular}


Table A.1.: Identifications, basic parameters, $\mathrm{pEW}_{\mathrm{H} \alpha}^{\prime}$ values, and normalised $\mathrm{H} \alpha$ luminosities of the analysed stars (cont.)

\begin{tabular}{|c|c|c|c|c|c|c|c|c|c|c|c|}
\hline No. & Karmn & Name & $\mathrm{Gl} / \mathrm{GJ}$ & $\mathrm{SpT}$ & Ref. & $\begin{array}{c}v \sin i \\
{\left[\mathrm{~km} \mathrm{~s}^{-1}\right]}\end{array}$ & Ref. & $\begin{array}{l}P_{\text {rot }} \\
\text { [d] }\end{array}$ & Ref. & $\begin{array}{c}\mathrm{pEW}_{\mathrm{H} \alpha}^{\prime} \\
{[\AA]}\end{array}$ & $\log \left(L_{\mathrm{H} \alpha} / L_{\mathrm{bol}}\right)$ \\
\hline 318 & $\mathrm{~J} 22468+443$ & EV Lac & 873 & M3.5 V & PMSU & $5.9 \pm 0.1$ & Fou18 & $4.38 \pm 0.03$ & DA19 & $-5.098 \pm 0.029$ & $-3.64 \pm 0.01$ \\
\hline 319 & $\mathrm{~J} 22503-070$ & BD-07 5871 & 875 & $\mathrm{M} 0.5 \mathrm{~V}$ & Gra03 & $<2$ & Rein18 & & 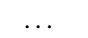 & $0.003 \pm 0.010$ & \\
\hline 320 & $\mathrm{~J} 22518+317$ & GT Peg & 875.1 & M3.0 V & PMSU & $13.2 \pm 0.9$ & Fou18 & $1.63 \pm 0.01$ & DA19 & $-5.186 \pm 0.021$ & $-3.55 \pm 0.01$ \\
\hline 321 & $\mathrm{~J} 22532-142$ & IL Aqr & 876 & $\mathrm{M} 4.0 \mathrm{~V}$ & PMSU & $<2$ & Rein18 & $81 \pm 0.8$ & DA19 & $0.054 \pm 0.011$ & $\ldots$ \\
\hline 322 & $\mathrm{~J} 22559+178$ & StKM 1-2065 & 4306 & $\mathrm{M} 1.0 \mathrm{~V}$ & PMSU & $<2$ & Rein18 & $27 \pm 2.5$ & SM18 & $-0.032 \pm 0.009$ & $\ldots$ \\
\hline 323 & $\mathrm{~J} 22565+165$ & HD 216899 & 880 & M1.5 V & PMSU & $<2$ & Rein18 & $39.5 \pm 0.2$ & DA19 & $0.065 \pm 0.014$ & \\
\hline 324 & $\mathrm{~J} 23064-050$ & 2MUCD 12171 (Trappist-1) & $\ldots$ & $\mathrm{M} 8.0 \mathrm{~V}$ & Schm07 & $<2$ & Rein18 & $3.304 \pm 0.011$ & DA19 & $-2.49 \pm 0.14$ & $-4.79 \pm 0.03$ \\
\hline 325 & $\mathrm{~J} 23113+085$ & NLTT 56083 & $\ldots$ & M3.5 V & Lep13 & $<2$ & Rein18 & & & $0.013 \pm 0.013$ & $\ldots$ \\
\hline 326 & $\mathrm{~J} 23216+172$ & LP 462-027 & 4333 & $\mathrm{M} 4.0 \mathrm{~V}$ & PMSU & $<2$ & Rein18 & $74.7 \pm 0.7$ & DA19 & $0.026 \pm 0.011$ & $\ldots$ \\
\hline 327 & $\mathrm{~J} 23245+578$ & $\mathrm{BD}+572735$ & 895 & $\mathrm{M} 1.0 \mathrm{~V}$ & PMSU & $<2$ & Rein18 & $\ldots$ & $\ldots$ & $-0.022 \pm 0.011$ & $\ldots$ \\
\hline 328 & $\mathrm{~J} 23340+001$ & GJ 899 & 899 & $\mathrm{M} 2.5 \mathrm{~V}$ & PMSU & $<2$ & Rein18 & $\ldots$ & $\ldots$ & $0.068 \pm 0.009$ & $\ldots$ \\
\hline 329 & $\mathrm{~J} 23351-023$ & GJ 1286 & 1286 & M5.5 V & PMSU & $<2$ & Rein18 & & & $-0.472 \pm 0.009$ & $-4.91 \pm 0.01$ \\
\hline 330 & $\mathrm{~J} 23381-162$ & G 273-093 & 4352 & $\mathrm{M} 2.0 \mathrm{~V}$ & PMSU & $2.1 \pm 1.2$ & Fou18 & 61.66 & Wat06 & $0.017 \pm 0.011$ & 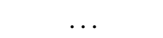 \\
\hline 331 & $\mathrm{~J} 23419+441$ & HH And & 905 & M5.0 V & AF15 & $<2$ & Rein18 & $106 \pm 6$ & DA19 & $-0.313 \pm 0.014$ & $-5.03 \pm 0.02$ \\
\hline 332 & $\mathrm{~J} 23431+365$ & GJ 1289 & 1289 & $\mathrm{M} 4.0 \mathrm{~V}$ & PMSU & $<2$ & Rein18 & $83.6 \pm 7$ & DA19 & $-0.943 \pm 0.009$ & $-4.41 \pm 0.01$ \\
\hline 333 & $\mathrm{~J} 23492+024$ & BR Psc & 908 & M1.0 V & PMSU & $<2$ & Rein18 & $49.9 \pm 3.5$ & SM18 & $-0.031 \pm 0.014$ & $\ldots$ \\
\hline 334 & $\mathrm{~J} 23505-095$ & LP 763-012 & 4367 & $\mathrm{M} 4.0 \mathrm{~V}$ & PMSU & $<2$ & Rein18 & & $\ldots$ & $-0.040 \pm 0.009$ & $\ldots$ \\
\hline 335 & $\mathrm{~J} 23548+385$ & RX J2354.8+3831 & $\ldots$ & $\mathrm{M} 4.0 \mathrm{~V}$ & Lep13 & $3.6 \pm 1.5$ & Rein18 & $4.70 \pm 0.04$ & DA19 & $-3.35 \pm 0.05$ & $-3.86 \pm 0.01$ \\
\hline 336 & $\mathrm{~J} 23556-061$ & GJ 912 & 912 & $\mathrm{M} 2.5 \mathrm{~V}$ & PMSU & $<2$ & Rein18 & $\ldots$ & $\ldots$ & $0.025 \pm 0.010$ & $\ldots$ \\
\hline 337 & $\mathrm{~J} 23585+076$ & Wolf 1051 & 4383 & $\mathrm{M} 3.0 \mathrm{~V}$ & PMSU & $<2$ & Rein18 & $\ldots$ & $\ldots$ & $0.048 \pm 0.009$ & $\ldots$ \\
\hline
\end{tabular}




\section{B. Periodograms}

The periodograms of the four stars analysed in Sect. 5.2 are collected in this appendix.

In Fig. B.5 for Ross 318, Fig. B.6 for YZ CMi, Fig. B.7 for TYC 3529-1437-1, and Fig. B.8 for EV Lac, rolling GLS periodograms of $\mathrm{pEW}_{\mathrm{H} \alpha}^{\prime}$ (left), TiO 7050 index (centre), and RV VIS (right) are shown. Each colour-coded stripe is a GLS periodogram of a subset of 21 consecutive data points. Its position on the y-axis corresponds to the median barycentric Julian date (BJD) of the subset. Subsets that span more than $180 \mathrm{~d}$ are shown in lighter colour shades.

On top of each rolling periodogram, the GLS periodogram calculated from the full data set is shown for comparison. On the right side of each rolling periodogram, the individual data points are shown. The dashed line marks the average value, while the dotted lines enclose the $2 \sigma$ interval around the average. Data points outside of the $2 \sigma$ interval are not used for calculating the periodograms and are shown in grey. Data points outside the date range are shown at the beginning of the date range and marked with an arrow. 

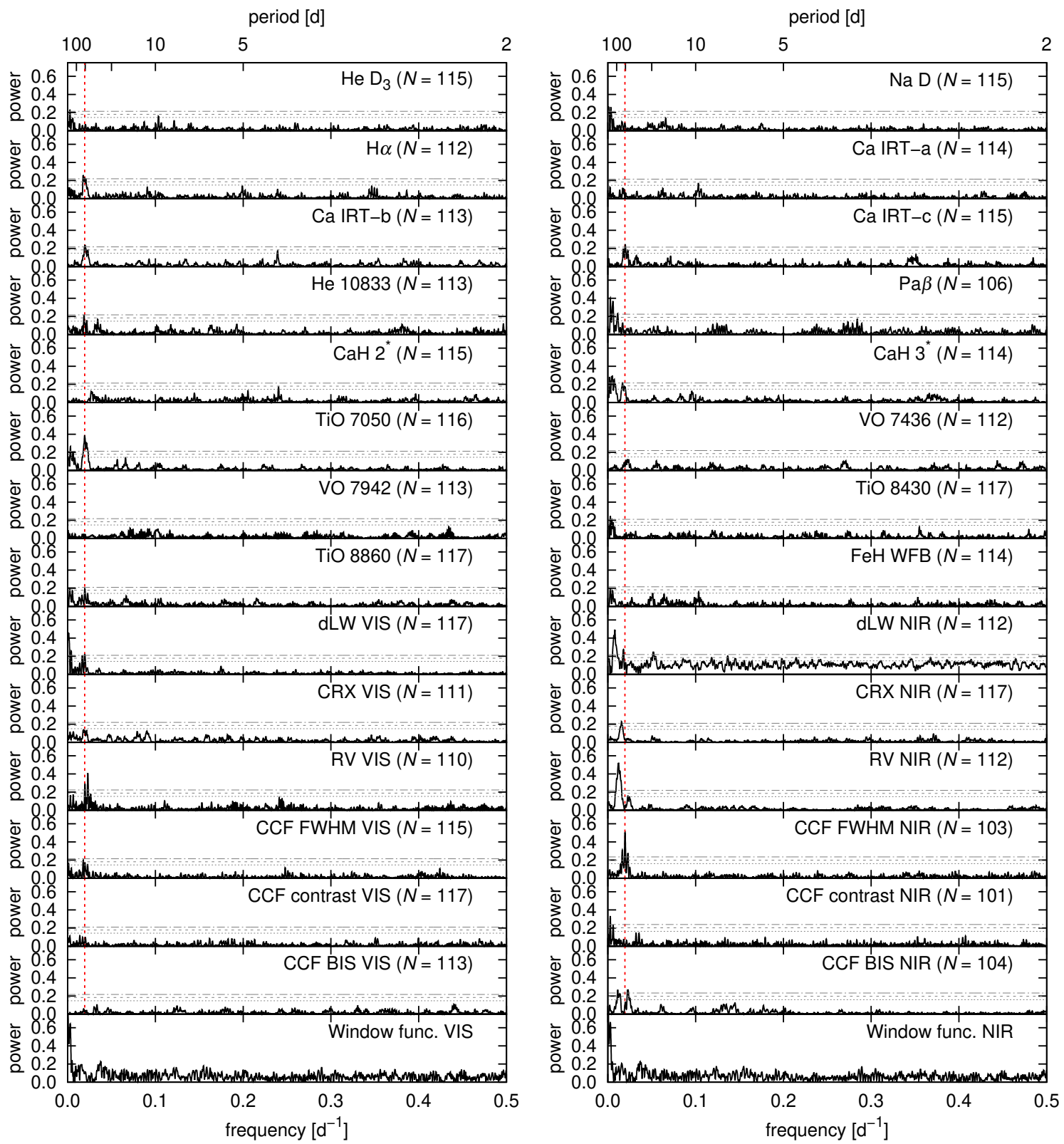

Figure B.1.: GLS periodograms of indicators and window functions for Ross 318 . Horizontal lines indicate the analytical 10\% (dotted), $1 \%$ (dashed), and $0.1 \%$ (dash-dotted) false alarm probability levels. $N$ is the number of used data points after a $2 \sigma$ clipping for each indicator. The red dotted line marks the rotation frequency $f_{\text {rot }}=0.0194 \mathrm{~d}^{-1}\left(P_{\text {rot }}=51.5 \mathrm{~d}\right)$. 

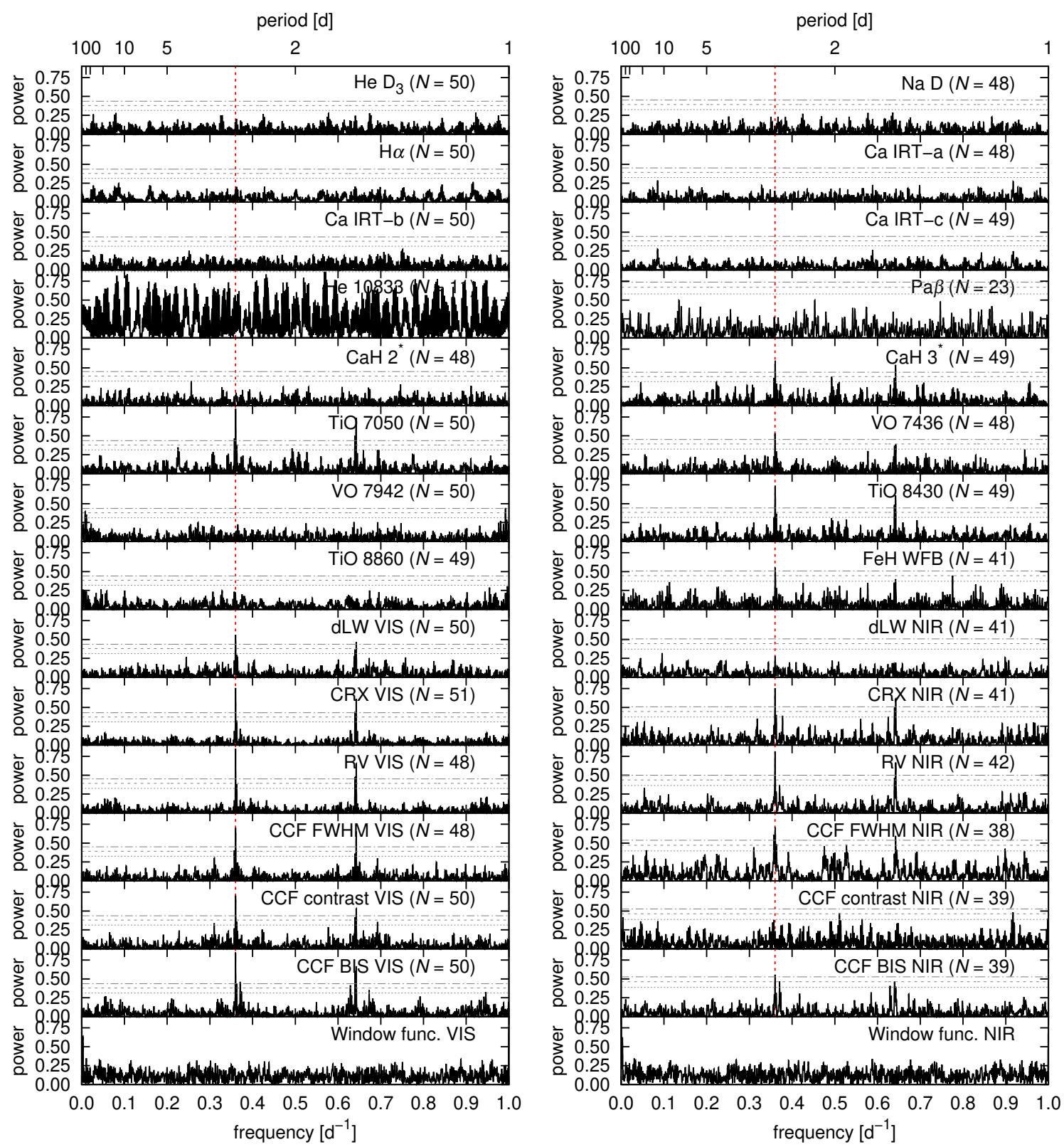

Figure B.2.: Same as Fig. B.1, but for YZ CMi. The red dotted line marks the rotation frequency $f_{\text {rot }}=0.36 \mathrm{~d}^{-1}\left(P_{\text {rot }}=2.78 \mathrm{~d}\right)$. 

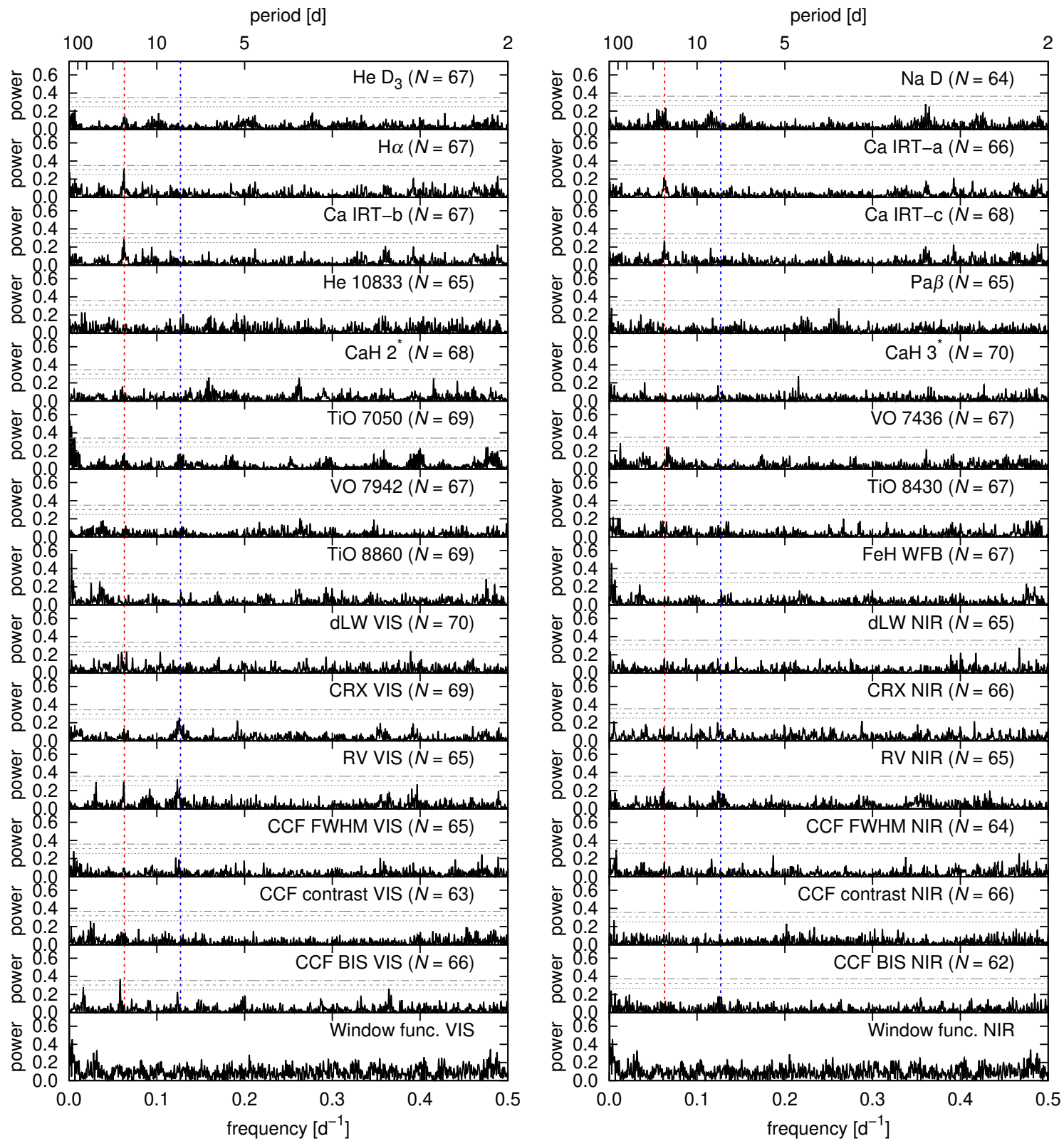

Figure B.3.: Same as Fig. B.1, but for TYC 3529-1437-1. The red dotted line marks the rotation frequency $f_{\text {rot }}=0.063 \mathrm{~d}^{-1}\left(P_{\text {rot }}=15.8 \mathrm{~d}\right)$, the blue dotted line marks its first harmonic $2 f_{\text {rot }}$. 

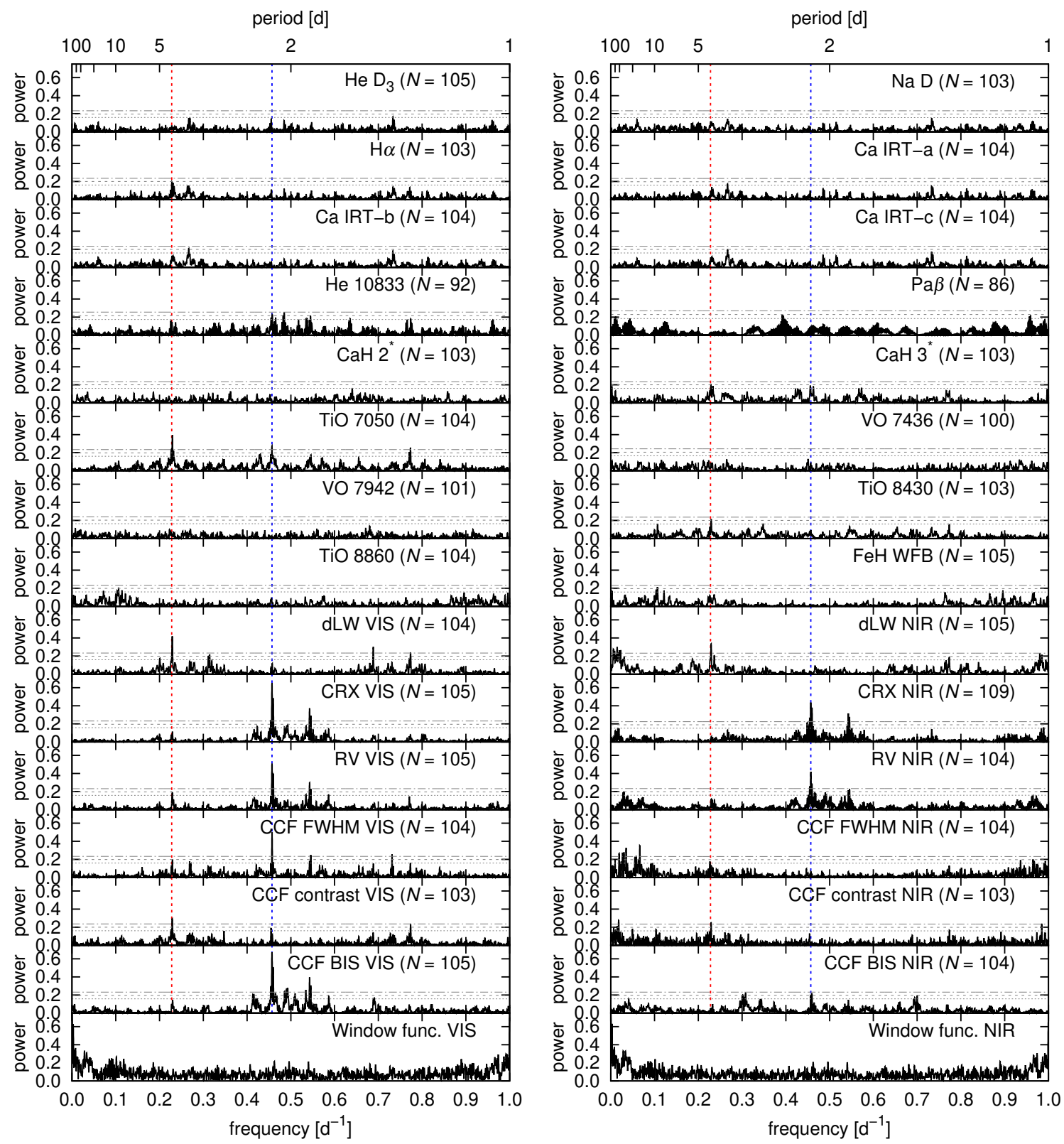

Figure B.4.: Same as Fig. B.1, but for EV Lac. The red dotted line marks the rotation frequency $f_{\text {rot }}=0.228 \mathrm{~d}^{-1}\left(P_{\text {rot }}=4.38 \mathrm{~d}\right)$, the blue dotted line marks its first harmonic $2 f_{\text {rot }}$. 


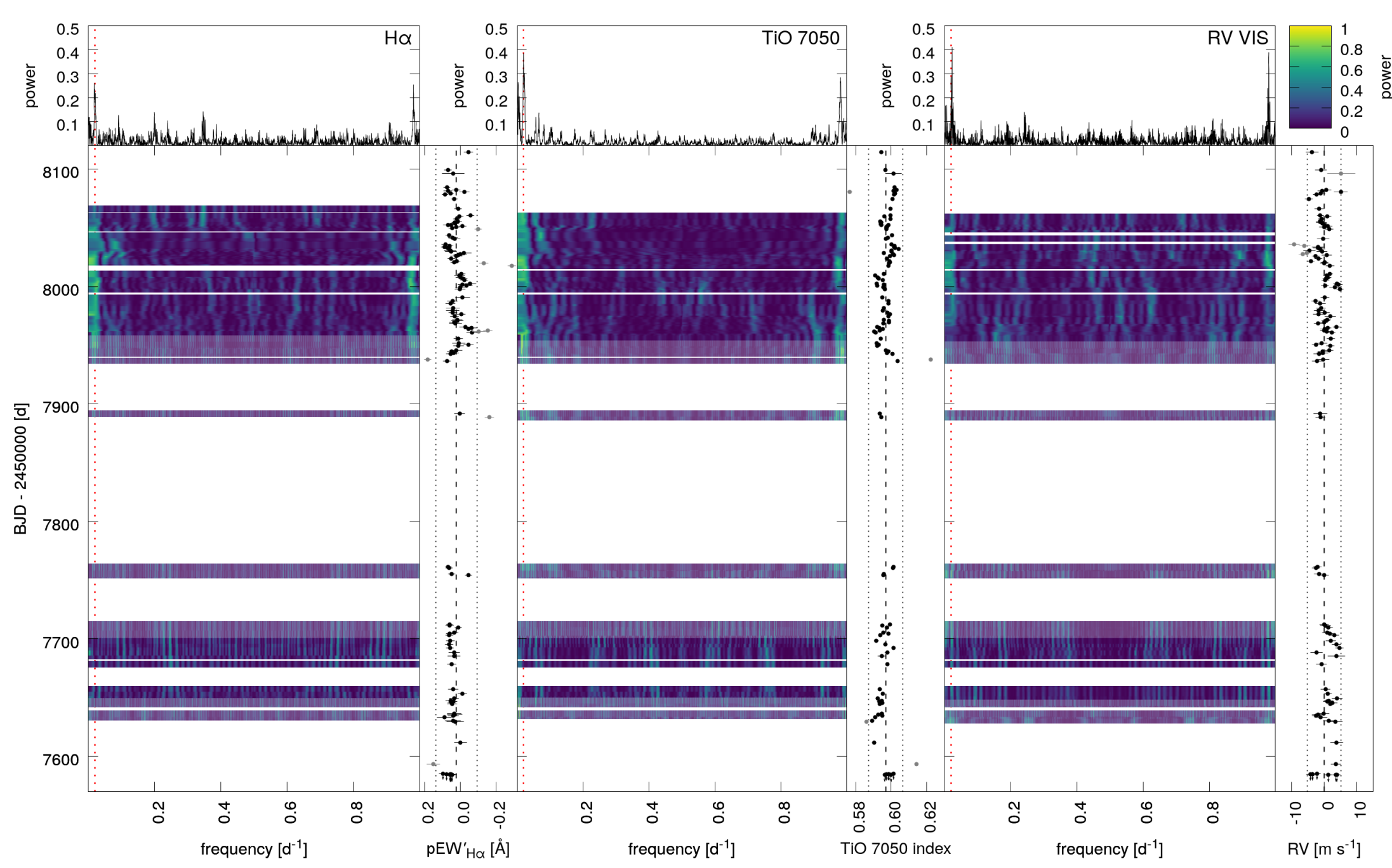

Figure B.5.: Rolling GLS periodograms for Ross 318 as described in Appendix B. The red dotted line marks $f_{\text {rot }}$. 


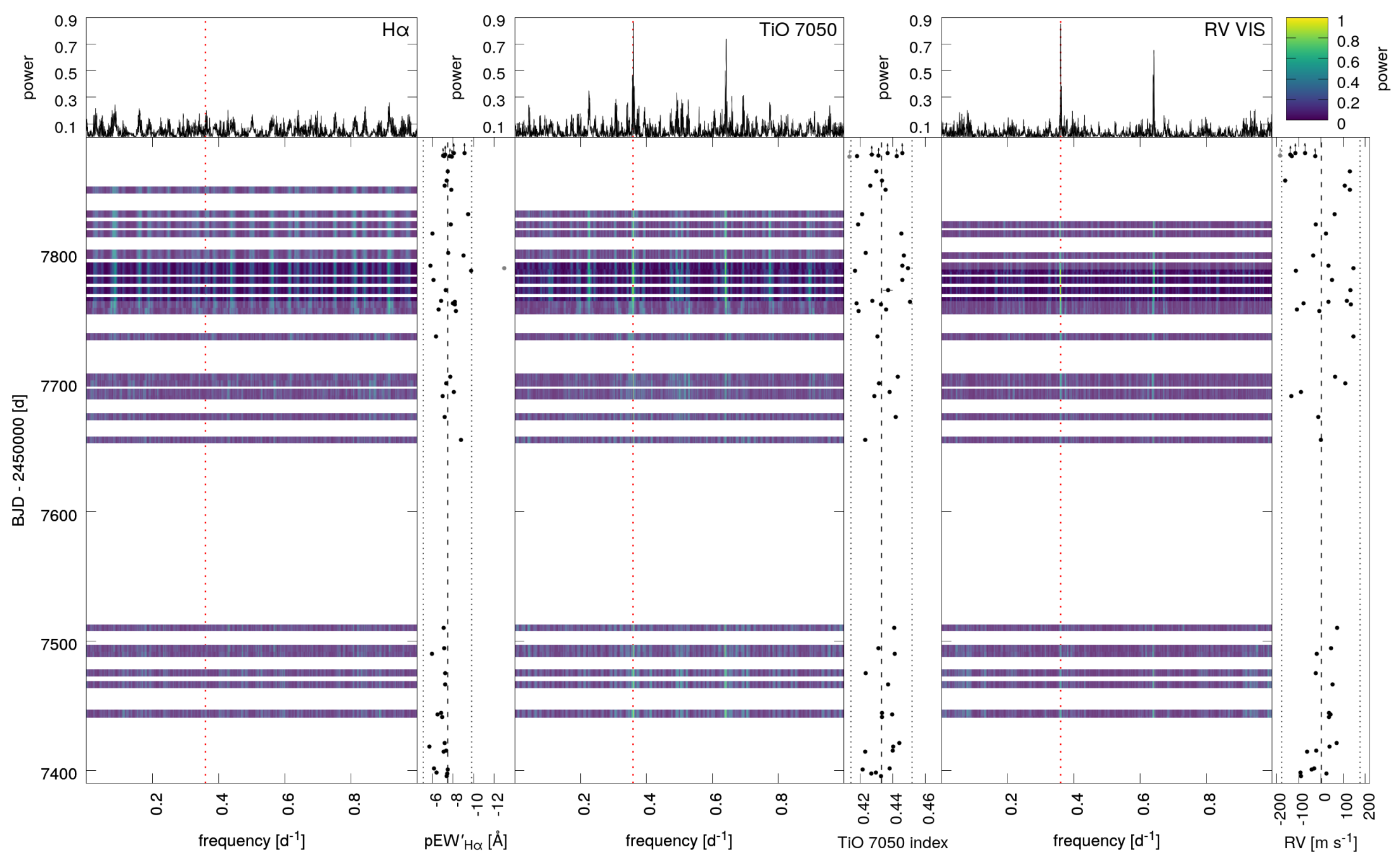

Figure B.6.: Rolling GLS periodograms for YZ CMi as described in Appendix B. The red dotted line marks $f_{\text {rot }}$. 


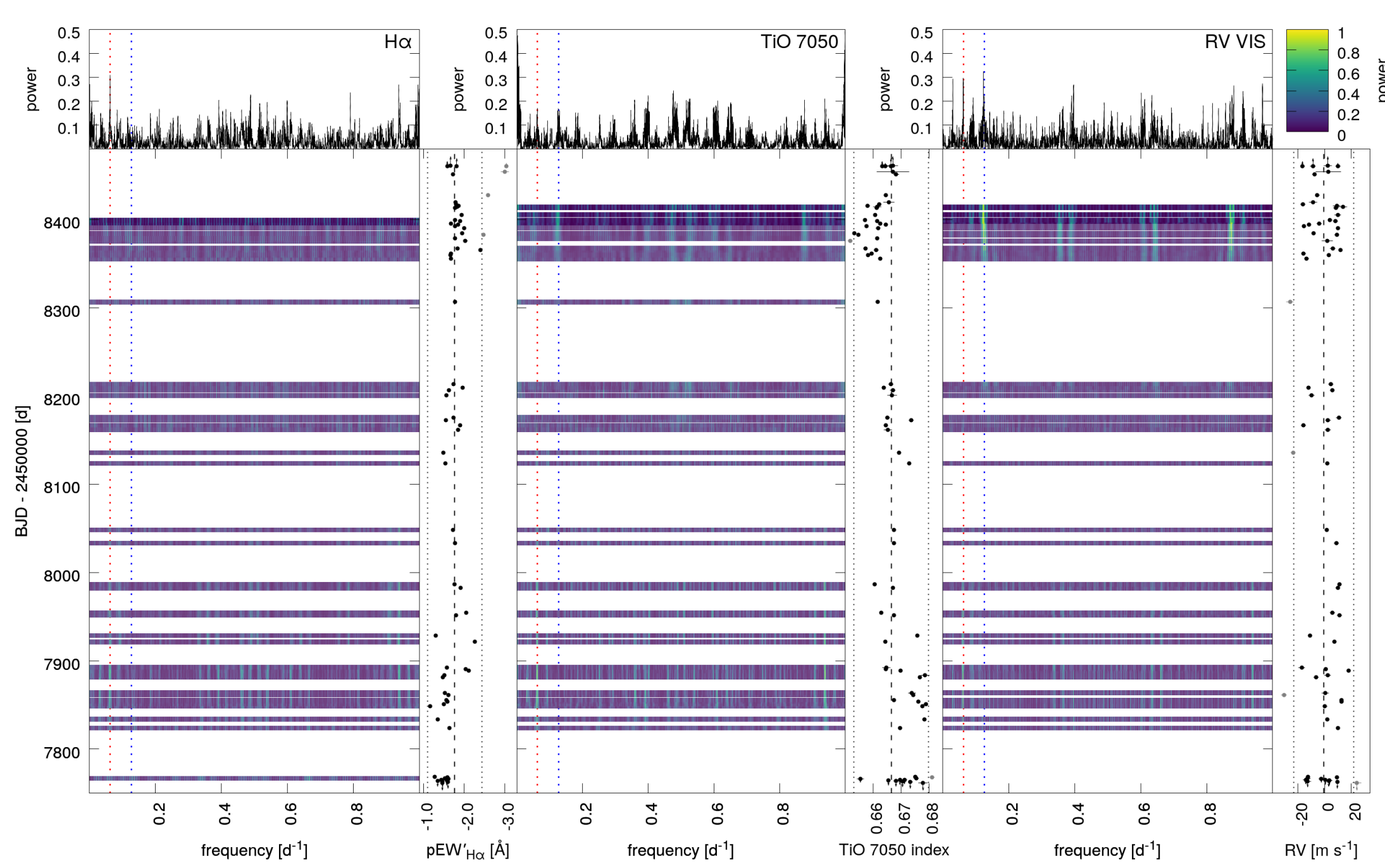

Figure B.7.: Rolling GLS periodograms for TYC 3529-1437-1 as described in Appendix B. The dotted lines mark $f_{\text {rot }}$ (red) and $2 f_{\text {rot }}$ (blue). 


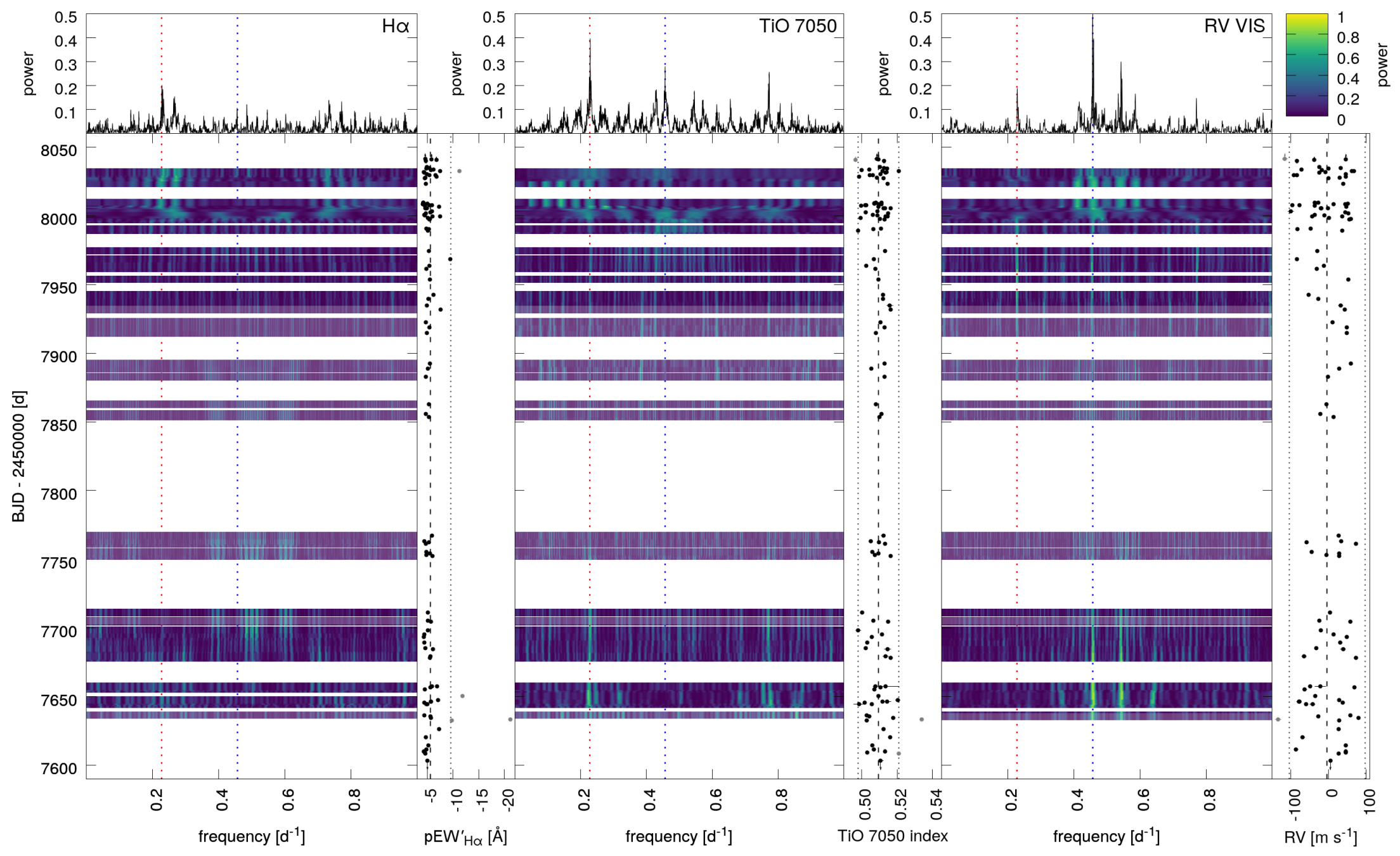

Figure B.8.: Rolling GLS periodograms for EV Lac as described in Appendix B. The dotted lines mark $f_{\text {rot }}$ (red) and $2 f_{\text {rot }}$ (blue). 



\section{Bibliography}

Adelberger, E. G., García, A., Robertson, R. G. H. et al. (2011) Solar fusion cross sections. II. The pp chain and CNO cycles. Reviews of Modern Physics, 83(1), 195-246.

Alekseev, I. Y. \& Kozhevnikova, A. V. (2017) Long-term variations in the spottedness of BY Dra M dwarfs. Astronomy Reports, 61(3), 221-232.

Alfvén, H. (1947) Magneto hydrodynamic waves, and the heating of the solar corona. MNRAS, 107, 211.

Allard, F., Hauschildt, P. H., Alexander, D. R., Tamanai, A. \& Schweitzer, A. (2001) The Limiting Effects of Dust in Brown Dwarf Model Atmospheres. ApJ, 556(1), 357-372.

Allart, R., Bourrier, V., Lovis, C. et Al. (2018) Spectrally resolved helium absorption from the extended atmosphere of a warm Neptune-mass exoplanet. Science, 362(6421), 1384-1387.

Alonso-Floriano, F. J., Morales, J. C., Caballero, J. A. et al. (2015) CARMENES input catalogue of M dwarfs. I. Low-resolution spectroscopy with CAFOS. A\&A, 577, A128.

Alonso-Floriano, F. J., Sánchez-López, A., Snellen, I. A. G. et Al. (2019) Multiple water band detections in the CARMENES near-infrared transmission spectrum of HD 189733 b. A\&A, 621, A74.

Alvarado-Gómez, J. D., Drake, J. J., Fraschetti, F. et al. (2020) Tuning the Exospace Weather Radio for Stellar Coronal Mass Ejections. ApJ, 895(1), 47.

Andretta, V., Doyle, J. G. \& Byrne, P. B. (1997) The NaI $\lambda \lambda 5890,5896$ resonance doublet as chromospheric diagnostics in M dwarfs. AEA A, 322, 266-279.

Anglada-Escudé, G., Arriagada, P., Tuomi, M. et al. (2014) Two planets around Kapteyn's star: a cold and a temperate super-Earth orbiting the nearest halo red dwarf. MNRAS, 443, L89-L93.

Anglada-Escudé, G., Tuomi, M., Arriagada, P. et al. (2016) No Evidence for Activity Correlations in the Radial Velocities of Kapteyn's Star. ApJ, 830(2), 74.

Artigau, É., Malo, L., Doyon, R. et al. (2018) Optical and Near-infrared Radial Velocity Content of M Dwarfs: Testing Models with Barnard's Star. AJ, 155(5), 198.

Astudillo-Defru, N., Delfosse, X., Bonfils, X. et al. (2017) Magnetic activity in the HARPS M dwarf sample. The rotation-activity relationship for very low-mass stars through $\mathbf{R}_{H K} \cdot A \mathcal{E} A, \mathbf{6 0 0}, \mathrm{A} 13$.

Ayres, T. R., Marstad, N. C. \& Linsky, J. L. (1981) Outer atmospheres of cool stars. IX. A survey of ultraviolet emission from F-K dwarfs and giants with the IUE. ApJ, 247, 545-559.

Bавсоск, H. W. (1961) The Topology of the Sun's Magnetic Field and the 22-YEAR Cycle. ApJ, 133, 572.

Baliunas, S. L., Donahue, R. A., Soon, W. H. et al. (1995) Chromospheric Variations in Main-Sequence Stars. II. ApJ, 438, 269. 
Baranne, A., Queloz, D., Mayor, M. Et AL. (1996) ElODIE: A spectrograph for accurate radial velocity measurements. AEFAS, 119, 373-390.

Barnes, J. R., Collier Cameron, A., Donati, J. F. et Al. (2005) The dependence of differential rotation on temperature and rotation. MNRAS, 357(1), L1-L5.

Barnes, J. R., James, D. J. \& Collier Cameron, A. (2002) The boundaries of Doppler imaging: Starspot patterns on M dwarfs. Astronomische Nachrichten, 323, 333-335.

Barnes, J. R., Jeffers, S. V., Haswell, C. A. et al. (2017) Surprisingly different star-spot distributions on the near equal-mass equal-rotation-rate stars in the M dwarf binary GJ 65 AB. MNRAS, 471(1), 811-823.

Barnes, J. R., Jeffers, S. V., Jones, H. R. A. et Al. (2015) Starspot Distributions on Fully Convective M Dwarfs: Implications for Radial Velocity Planet Searches. ApJ, 812(1), 42.

Barnes, R. \& Heller, R. (2013) Habitable Planets Around White and Brown Dwarfs: The Perils of a Cooling Primary. Astrobiology, 13(3), 279-291.

Baroch, D., Morales, J. C., Ribas, I. Et al. (2018) The CARMENES search for exoplanets around M dwarfs. Nine new double-line spectroscopic binary stars. AEA, 619, A32.

Basri, G. \& Nguyen, H. T. (2018) Double Dipping: A New Relation between Stellar Rotation and Starspot Activity. ApJ, 863(2), 190.

Basri, G. \& Shah, R. (2020) The Information Content in Analytic Spot Models of Broadband Precision Light Curves. II. Spot Distributions and Lifetimes and Global and Differential Rotation. ApJ, 901(1), 14.

Bauer, F. F., Reiners, A., Beeck, B. \& Jeffers, S. V. (2018) The influence of convective blueshift on radial velocities of F, G, and $\mathrm{K}$ stars. A $\mathcal{E} A, \mathbf{6 1 0}, \mathrm{A} 52$.

Bauer, F. F., Zechmeister, M., Kaminski, A. et AL. (2020) The CARMENES search for exoplanets around $\mathrm{M}$ dwarfs. Measuring precise radial velocities in the near infrared: The example of the super-Earth $\mathrm{CD}$ Cet b. A\&A, 640, A50.

Bauer, F. F., Zechmeister, M. \& Reiners, A. (2015) Calibrating echelle spectrographs with Fabry-Pérot etalons. A\&A, 581, A117.

Beckers, J. M. \& Nelson, G. D. (1978) Some comments on the limb shift of solar lines. II: The effect of granular motions. Sol. Phys., 58(2), 243-261.

Bell, K. J., Hilton, E. J., Davenport, J. R. A. et Al. (2012) H $\alpha$ Emission Variability in Active M Dwarfs. $P A S P, \mathbf{1 2 4}, 14$.

Benneke, B., Werner, M., Petigura, E. et Al. (2017) Spitzer Observations Confirm and Rescue the Habitable-zone Super-Earth K2-18b for Future Characterization. ApJ, 834(2), 187.

Benz, A. O. (2017) Flare Observations. Living Reviews in Solar Physics, 14(1), 2.

Benz, A. O., Conway, J. \& Gudel, M. (1998) First VLBI images of a main-sequence star. AEA A, 331, 596600.

Benz, A. O. \& GüDEL, M. (1994) X-ray/microwave ratio of flares and coronae. AEFA, 285, 621-630.

Benz, A. O. \& Güdel, M. (2010) Physical Processes in Magnetically Driven Flares on the Sun, Stars, and Young Stellar Objects. ARA\&A, 48, 241-287. 
Berdyugina, S. V. (2005) Starspots: A Key to the Stellar Dynamo. Living Reviews in Solar Physics, 2(1), 8.

Berdyugina, S. V. \& SolankI, S. K. (2002) The molecular Zeeman effect and diagnostics of solar and stellar magnetic fields. I. Theoretical spectral patterns in the Zeeman regime. AEA, 385, 701-715.

BiermanN, L. (1946) Zur Deutung der chromosphärischen Turbulenz und des Exzesses der UV-Strahlung der Sonne. Naturwissenschaften, 33(4), 118-119.

BieRmanN, L. (1951) Kometenschweife und solare Korpuskularstrahlung. ZAp, 29, 274.

Bond, H. E., Mullan, D. J., O’Brien, M. S. \& Sion, E. M. (2001) Detection of Coronal Mass Ejections in V471 Tauri with the Hubble Space Telescope. ApJ, 560(2), 919-927.

Bopp, B. W. \& Evans, D. S. (1973) The spotted flare stars BY Dra and CC Eri: a model for the spots and some astrophysical implications. MNRAS, 164, 343-356.

Boro Saikia, S., JefFers, S. V., Morin, J. et al. (2016) A solar-like magnetic cycle on the mature K-dwarf 61 Cygni A (HD 201091). A\&A, 594, A29.

Boro Saikia, S., Marvin, C. J., Jeffers, S. V. et al. (2018) Chromospheric activity catalogue of 4454 cool stars. Questioning the active branch of stellar activity cycles. $A \& A, \mathbf{6 1 6}, \mathrm{A} 108$.

Bowen, I. S. \& Vaughan, A. H., J. (1973) “Nonobjective” Gratings. PASP, 85(504), 174.

Boyajian, T. S., von Braun, K., van Belle, G. et al. (2012) Stellar Diameters and Temperatures. II. Mainsequence K- and M-stars. ApJ, 757(2), 112.

Browning, M. K. (2008) Simulations of Dynamo Action in Fully Convective Stars. ApJ, 676(2), 1262-1280.

Browning, M. K., Basri, G., Marcy, G. W., West, A. A. \& Zhang, J. (2010) Rotation and Magnetic Activity in a Sample of M-Dwarfs. AJ, 139(2), 504-518.

Burgasser, A. J., Geballe, T. R., Leggett, S. K., Kirkpatrick, J. D. \& Golimowski, D. A. (2006) A Unified Near-Infrared Spectral Classification Scheme for T Dwarfs. ApJ, 637(2), 1067-1093.

Burnight, T. R. (1949) Soft X-radiation in the upper atmosphere. Phys. Rev., 76, 165.

Busà, I., Aznar Cuadrado, R., Terranegra, L., Andretta, V. \& Gomez, M. T. (2007) The Ca II infrared triplet as a stellar activity diagnostic. II. Test and calibration with high resolution observations. AEA, 466(3), 1089-1098.

Caballero, J. A., Cortés-Contreras, M., Alonso-Floriano, F. J. et al. (2016) Carmencita, The CARMENES Input Catalogue of Bright, Nearby M Dwarfs. In 19th Cambridge Workshop on Cool Stars, Stellar Systems, and the Sun (CS19), edited by G. A. Feiden, Zenodo.

Campbell, B. \& Walker, G. A. H. (1979) Precision radial velocities with an absorption cell. PASP, 91, $540-545$.

Cannon, A. J. \& Pickering, E. C. (1901) Spectra of bright southern stars photographed with the 13-inch Boyden telescope as part of the Henry Draper Memorial. Annals of Harvard College Observatory, 28, 129-P.6.

Carrington, R. C. (1858) On the Distribution of the Solar Spots in Latitudes since the Beginning of the Year 1854, with a Map. MNRAS, 19, 1-3.

Carrington, R. C. (1859) Description of a Singular Appearance seen in the Sun on September 1, 1859. MNRAS, 20, 13-15. 
Catalano, S., Biazzo, K., Frasca, A. \& Marilli, E. (2002) Measuring starspot temperature from line depth ratios. I. The method. A\&A, 394, 1009-1021.

Catalano, S. \& Marilli, E. (1983) CA II chromospheric emission and rotation of main sequence stars. $A \mathcal{E} A, \mathbf{1 2 1}, 190-197$.

Catura, R. C., Acton, L. W. \& Johnson, H. M. (1975) Evidence for X-ray emission from Capella. ApJL, 196, L47-L49.

Chabrier, G. \& Baraffe, I. (1997) Structure and evolution of low-mass stars. AEFA, 327, 1039-1053.

Chabrier, G. \& Baraffe, I. (2000) Theory of Low-Mass Stars and Substellar Objects. ARAEA, 38, 337-377.

Chabrier, G. \& Küker, M. (2006) Large-scale $\alpha \hat{2}$-dynamo in low-mass stars and brown dwarfs. $A \mathcal{E} A$, 446(3), 1027-1037.

Chapman, G. A. \& Sheeley, N. R., J. (1968) The Photospheric Network. Sol. Phys., 5(4), 442-461.

Charbonneau, P. (2020) Dynamo models of the solar cycle. Living Reviews in Solar Physics, 17(1), 4.

Chen, J. (2017) Physics of erupting solar flux ropes: Coronal mass ejections (CMEs) - Recent advances in theory and observation. Physics of Plasmas, 24(9), 090501.

Cho, K., Lee, J., Chae, J. ET Al. (2016) Strong Blue Asymmetry in H $\alpha$ Line as a Preflare Activity. Sol. Phys., 291(8), 2391-2406.

Chugainov, P. F. (1966) On the Variability of HD 234677. Information Bulletin on Variable Stars, $122,1$.

Cohen, O., Drake, J. J., Glocer, A. et al. (2014) Magnetospheric Structure and Atmospheric Joule Heating of Habitable Planets Orbiting M-dwarf Stars. ApJ, 790(1), 57.

Cortés-Contreras, M., Domínguez-Fernández, A. J., Caballero, J. A. et al. (in prep.) CARMENES input catalogue of M dwarfs. VI. Kinematics in the solar neighbourhood. AEFA, in prep.

Cram, L. E. \& Giampapa, M. S. (1987) Formation of Chromospheric Lines in Cool Dwarf Stars. ApJ, 323, 316.

Cram, L. E. \& Mullan, D. J. (1979) Model chromospheres of flare stars.I. Balmer-line profiles. ApJ, 234, 579-587.

Crosley, M. K. \& Osten, R. A. (2018) Low-frequency Radio Transients on the Active M-dwarf EQ Peg and the Search for Coronal Mass Ejections. ApJ, 862(2), 113.

Cuntz, M., SaAr, S. H. \& Musielak, Z. E. (2000) On Stellar Activity Enhancement Due to Interactions with Extrasolar Giant Planets. ApJL, 533(2), L151-L154.

Davenport, J. R. A. (2016) The Kepler Catalog of Stellar Flares. ApJ, 829(1), 23.

David, T. J., Hillenbrand, L. A., Petigura, E. A. et al. (2016) A Neptune-sized transiting planet closely orbiting a 5-10-million-year-old star. Nature, 534(7609), 658-661.

Dekker, H., D’Odorico, S., Kaufer, A., Delabre, B. \& Kotzlowski, H. (2000) Design, construction, and performance of UVES, the echelle spectrograph for the UT2 Kueyen Telescope at the ESO Paranal Observatory. In Proc. SPIE, edited by M. Iye \& A. F. Moorwood, vol. 4008 of Society of Photo-Optical Instrumentation Engineers (SPIE) Conference Series, pp. 534-545. 
Delfosse, X., Forveille, T., Perrier, C. \& Mayor, M. (1998) Rotation and chromospheric activity in field

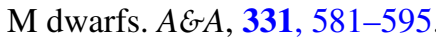

Delisle, J. B., Ségransan, D., Dumusque, X. et Al. (2018) The HARPS search for southern extra-solar planets. XLIII. A compact system of four super-Earth planets orbiting HD 215152. A\&A, 614, A133.

Desort, M., Lagrange, A. M., Galland, F., Udry, S. \& Mayor, M. (2007) Search for exoplanets with the radial-velocity technique: quantitative diagnostics of stellar activity. A\&A, 473(3), 983-993.

Díaz, R. F., Cincunegui, C. \& Mauas, P. J. D. (2007) The NaI D resonance lines in main-sequence late-type stars. MNRAS, 378(3), 1007-1018.

Díez Alonso, E., Caballero, J. A., Montes, D. et al. (2019) CARMENES input catalogue of M dwarfs. IV. New rotation periods from photometric time series. $A \mathcal{E} A$, 621, A126.

Dobler, W., Stix, M. \& Brandenburg, A. (2006) Magnetic Field Generation in Fully Convective Rotating Spheres. ApJ, 638(1), 336-347.

Donahue, R. A., SaAr, S. H. \& Baliunas, S. L. (1996) A Relationship between Mean Rotation Period in Lower Main-Sequence Stars and Its Observed Range. ApJ, 466, 384.

Donati, J. F. \& Collier Cameron, A. (1997) Differential rotation and magnetic polarity patterns on AB Doradus. MNRAS, 291(1), 1-19.

Donati, J.-F., Kouach, D., Lacombe, M. et al. (2018) SPIRou: A NIR Spectro-polarimeter/High-Precision Velocimeter for the CFHT. In Handbook of Exoplanets, Springer International Publishing, pp. 903-929.

Donati, J. F., Morin, J., Petit, P. et AL. (2008) Large-scale magnetic topologies of early M dwarfs. MNRAS, 390(2), 545-560.

Donati, J. F., Semel, M. \& Praderie, F. (1989) Zeeman-Doppler imaging of active stars. II. Numerical simulation and first observational results. $A \mathcal{E} A, 225,467-478$.

Dorn, R. J., Anglada-Escude, G., BaAde, D. ET Al. (2014) CRIRES+: Exploring the Cold Universe at High Spectral Resolution. The Messenger, 156, 7-11.

Dravins, D. (1982) Photospheric spectrum line asymmetries and wavelength shifts. ARA $\sigma A$, 20, 61-89.

Dumusque, X. (2018) Measuring precise radial velocities on individual spectral lines. I. Validation of the method and application to mitigate stellar activity. AEA, 620, A47.

Dumusque, X., Pepe, F., Lovis, C. Et AL. (2012) An Earth-mass planet orbiting $\alpha$ Centauri B. Nature, 491(7423), 207-211.

Dumusque, X., Udry, S., Lovis, C., Santos, N. C. \& Monteiro, M. J. P. F. G. (2011) Planetary detection limits taking into account stellar noise. I. Observational strategies to reduce stellar oscillation and granulation effects. $A \mathcal{E} A, \mathbf{5 2 5}, \mathrm{A} 140$.

Duncan, D. K., Vaughan, A. H., Wilson, O. C. et al. (1991) CA II H and K Measurements Made at Mount Wilson Observatory, 1966-1983. ApJS, 76, 383.

Durney, B. R., De Young, D. S. \& Roxburgh, I. W. (1993) On the Generation of the Largescale and Turbulent Magnetic Fields in the Solar Type Stars. Sol. Phys., 145(2), 207-225.

Eberhard, G. \& Schwarzschild, K. (1913) On the reversal of the calcium lines H and K in stellar spectra. ApJ, 38, 292-295. 
EdLÉn, B. (1943) Die Deutung der Emissionslinien im Spektrum der Sonnenkorona. ZAp, 22, 30.

Endl, M., Kürster, M., Rouesnel, F. et Al. (2003) Extrasolar Terrestrial Planets: Can We Detect Them Already? In Scientific Frontiers in Research on Extrasolar Planets, edited by D. Deming \& S. Seager, vol. 294 of Astronomical Society of the Pacific Conference Series, pp. 75-78.

Favata, F. \& Schmitt, J. H. M. M. (1999) Spectroscopic analysis of a super-hot giant flare observed on Algol by BeppoSAX on 30 August 1997. AEFA, 350, 900-916.

Fontenla, J. M., Linsky, J. L., Witbrod, J. et Al. (2016) Semi-empirical Modeling of the Photosphere, Chromosphere, Transition Region, and Corona of the M-dwarf Host Star GJ 832. ApJ, 830(2), 154.

Fouqué, P., Moutou, C., Malo, L. ET Al. (2018) SPIRou Input Catalogue: global properties of 440 M dwarfs observed with ESPaDOnS at CFHT. MNRAS, 475(2), 1960-1986.

FröHLich, C. (2002) Total solar irradiance variations since 1978. Advances in Space Research, 29(10), 1409-1416.

Fuhrmeister, B., Czesla, S., Hildebrandt, L. Et al. (2019a) The CARMENES search for exoplanets around M dwarfs. The He I triplet at $10830 \AA$ across the M dwarf sequence. A $\mathcal{F} A$, 632, A24.

Fuhrmeister, B., Czesla, S., Schmitt, J. H. M. M. et Al. (2018) The CARMENES search for exoplanets around $\mathrm{M}$ dwarfs. Wing asymmetries of $\mathrm{H} \alpha, \mathrm{Na}$ I D, and He I lines. A $\mathcal{F} A, \mathbf{6 1 5}, \mathrm{A} 14$.

Fuhrmeister, B., Czesla, S., Schmitt, J. H. M. M. et Al. (2019b) The CARMENES search for exoplanets around $\mathrm{M}$ dwarfs. Period search in $\mathrm{H} \alpha, \mathrm{Na}$ I D, and Ca II IRT lines. A $\mathcal{F} A, \mathbf{6 2 3}, \mathrm{A} 24$.

Fuhrmeister, B., Liefke, C., Schmitt, J. H. M. M. \& Reiners, A. (2008) Multiwavelength observations of a giant flare on CN Leonis. I. The chromosphere as seen in the optical spectra. A\&A, 487(1), 293-306.

Gaia Collaboration, Brown, A. G. A., Vallenari, A. et al. (2018) Gaia Data Release 2. Summary of the contents and survey properties. $A \mathcal{E} A, \mathbf{6 1 6}, \mathrm{A} 1$.

Gaidos, E., Mann, A. W., Kraus, A. L. \& Ireland, M. (2016) They are small worlds after all: revised properties of Kepler M dwarf stars and their planets. MNRAS, 457(3), 2877-2899.

GershberG, R. E. (1972) Some results of the cooperative photometric observations of the UV Cet-type flare stars in the years 1967 71. ApESS, 19(1), 75-92.

Gigoyan, K. S., Sinamyan, P. K., Engels, D. \& Mickaelian, A. M. (2010) Late-type stars found in the DFBS. Astrophysics, 53(1), 123-132.

Giridhar, S. (2010) Spectral Classification: Old and Contemporary. Astrophysics and Space Science Proceedings, 16, 165.

Gizis, J. E., Reid, I. N. \& Hawley, S. L. (2002) The Palomar/MSU Nearby Star Spectroscopic Survey. III. Chromospheric Activity, M Dwarf Ages, and the Local Star Formation History. AJ, 123(6), 3356-3369.

Gomes da Silva, J., Santos, N. C., Bonfils, X. et Al. (2011) Long-term magnetic activity of a sample of M-dwarf stars from the HARPS program. I. Comparison of activity indices. A $\mathcal{E} A, \mathbf{5 3 4}, \mathrm{A} 30$.

Gopalswamy, N., Thompson, W. T., Davila, J. M. et al. (2009) Relation Between Type II Bursts and CMEs Inferred from STEREO Observations. Sol. Phys., 259(1-2), 227-254.

Gray, R. O., Corbally, C. J., Garrison, R. F., McFadden, M. T. \& Robinson, P. E. (2003) Contributions to the Nearby Stars (NStars) Project: Spectroscopy of Stars Earlier than M0 within 40 Parsecs: The Northern Sample. I. AJ, 126(4), 2048-2059. 
Gray, R. O., Corbally, C. J., Garrison, R. F. et al. (2006) Contributions to the Nearby Stars (NStars) Project: Spectroscopy of Stars Earlier than M0 within 40 pc-The Southern Sample. AJ, 132(1), 161170.

GrIfFIN, R. \& GRIFFIN, R. (1973) On the possibility of determining stellar radial velocities to $0.01 \mathrm{~km} \mathrm{~s}^{-1}$. MNRAS, 162, 243-253.

Gringauz, K. I., Bezrukikh, V. V., Ozerov, V. D. \& RybchinskiI, R. E. (1962) The study of interplanetary ionized gas, high-energy electrons and corpuscular radiation of the sun, employing three-electrode charged particle traps on the second Soviet space rocket. Planet. Space Sci., 9(3), 103-107.

GüDEL, M. (2002) Stellar Radio Astronomy: Probing Stellar Atmospheres from Protostars to Giants. ARA $\mathcal{F} A, \mathbf{4 0}, 217-261$.

GüDEL, M. (2004) X-ray astronomy of stellar coronae. A $\mathcal{F} A$ Rev., 12(2-3), 71-237.

Güdel, M. \& Benz, A. O. (1993) X-Ray/Microwave Relation of Different Types of Active Stars. ApJL, 405, L63.

GüDEL, M. \& NAZÉ, Y. (2009) X-ray spectroscopy of stars. A E A Rev., 17(3), 309-408.

Guenther, E. W. \& Emerson, J. P. (1997) Spectrophotometry of flares and short time scale variations in weak line, and classical T Tauri stars in Chamaeleon. AEFA, 321, 803-810.

Haisch, B., Strong, K. T. \& Rodono, M. (1991) Flares on the Sun and other stars. ARAEA, 29, $275-324$.

Haisch, B. M., Slee, O. B., Siegman, B. C. et al. (1981) Simultaneous X-ray, ultraviolet, optical, and radio observations of the flare star Proxima Centauri. ApJ, 245, 1009-1017.

Hale, G. E. (1908) On the Probable Existence of a Magnetic Field in Sun-Spots. ApJ, 28, 315.

Hall, D. S. \& Henry, G. W. (1994) The Law of Starspot Lifetimes. International Amateur-Professional Photoelectric Photometry Communications, 55, 51.

HaLl, J. C. (2008) Stellar Chromospheric Activity. Living Reviews in Solar Physics, 5(1), 2.

Hardegree-Ullman, K. K., Cushing, M. C., Muirhead, P. S. \& Christiansen, J. L. (2019) Kepler Planet Occurrence Rates for Mid-type M Dwarfs as a Function of Spectral Type. AJ, 158(2), 75.

Harrell, F. E. \& Davis, C. E. (1982) A new distribution-free quantile estimator. Biometrika, 69(3), 635640.

Harrison, G. R. (1949) The production of Diffraction Gratings: II. Design of Echelle Gratings and Spectrographs. Journal of the Optical Society of America (1917-1983), 39(7), 522.

Hathaway, D. H. (2015) The Solar Cycle. Living Reviews in Solar Physics, 12(1), 4.

Hatzes, A. P. (2013) The Radial Velocity Detection of Earth-mass Planets in the Presence of Activity Noise: The Case of $\alpha$ Centauri Bb. ApJ, 770(2), 133.

Hawley, S. L., Gizis, J. E. \& Reid, I. N. (1996) The Palomar/MSU Nearby Star Spectroscopic Survey. II. The Southern M Dwarfs and Investigation of Magnetic Activity. AJ, 112, 2799.

Hawley, S. L. \& Pettersen, B. R. (1991) The Great Flare of 1985 April 12 on AD Leonis. ApJ, 378, 725.

Haywood, R. D., Collier Cameron, A., Queloz, D. et al. (2014) Planets and stellar activity: hide and seek in the CoRoT-7 system. MNRAS, 443(3), 2517-2531. 
Hearnshaw, J. B. (2009) Astronomical Spectrographs and their History. Cambridge University Press.

Hearnshaw, J. B. (2014) The Analysis of Starlight: Two Centuries of Astronomical Spectroscopy. 2nd edn., Cambridge University Press.

Henry, T. J., Jao, W.-C., Winters, J. G. et AL. (2018) The Solar Neighborhood XLIV: RECONS Discoveries within 10 parsecs. $A J, \mathbf{1 5 5}(6), 265$.

Henwood, R., Chapman, S. C. \& Willis, D. M. (2010) Increasing Lifetime of Recurrent Sunspot Groups Within the Greenwich Photoheliographic Results. Sol. Phys., 262(2), 299-313.

HerTzSPRUNG, E. (1911) Über die Verwendung photographischer effektiver Wellenlängen zur Bestimmung von Farbenäquivalenten. Publikationen des Astrophysikalischen Ob-servatoriums zu Potsdam, 22(63), $1-40$.

Heyvaerts, J. (1990) Coronal Heating by DC Currents. In Basic Plasma Processes on the Sun, edited by E. R. Priest \& V. Krishan, vol. 142, p. 207.

Hidalgo, D., Pallé, E., Alonso, R. et al. (2020) Three planets transiting the evolved star EPIC 249893012: a hot $8.8-\mathrm{M}_{\oplus}$ super-Earth and two warm 14.7 and $10.2-\mathrm{M}_{\oplus}$ sub-Neptunes. A\&A, 636, A89.

Hintz, D., Fuhrmeister, B., Czesla, S. et al. (2019) The CARMENES search for exoplanets around M dwarfs. Chromospheric modeling of M 2-3 V stars with PHOENIX. A\&A, 623, A136.

Hodgson, R. (1859) On a curious Appearance seen in the Sun. MNRAS, 20, 15-16.

Houdebine, E. R. (2012) Observation and modelling of main-sequence star chromospheres - XVIII. Observations of the Ca II resonance lines and $\mathrm{H} \alpha$ line for dM4 stars and dK5 stars. MNRAS, 421(4), 3189-3205.

Houdebine, E. R., Foing, B. H. \& Rodono, M. (1990) Dynamics of flares on late-type dMe stars. I. Flare mass ejections and stellar evolution. $A \mathcal{E} A, \mathbf{2 3 8}, 249$.

Houdebine, E. R., Junghans, K., Heanue, M. C. \& Andrews, A. D. (2009) Observation and modelling of main sequence star chromospheres. VIII. High resolution observations of $\mathrm{M}$ and $\mathrm{K}$ dwarf chromospheric lines. $A \mathcal{E} A, \mathbf{5 0 3}(3), 929-944$.

Houdebine, E. R. \& Mullan, D. J. (2015) Dynamics of Rotation in M Dwarfs: Indications for a Change in the Dynamo Regime in Stars at the Onset of Complete Convection. ApJ, 801(2), 106.

Huenemoerder, D. P., Ramsey, L. W. \& Buzasi, D. L. (1989) Titanium Oxide Variations in II Pegasi. AJ, 98, 2264.

Husser, T. O., Wende-von Berg, S., Dreizler, S. et al. (2013) A new extensive library of PHOENIX stellar atmospheres and synthetic spectra. $A \& A, \mathbf{5 5 3}, \mathrm{A} 6$.

Iснimoto, K. \& Kurokawa, H. (1984) H $\alpha$ Red Asymmetry of Solar Flares. Sol. Phys., 93(1), 105-121.

Jacquinot, P. (1954) The luminosity of spectrometers with prisms, gratings, or Fabry Perot etalons. Journal of the Optical Society of America (1917-1983), 44(10), 761.

Jefferies, S. M., McIntosh, S. W., Armstrong, J. D. et al. (2006) Magnetoacoustic Portals and the Basal Heating of the Solar Chromosphere. ApJL, 648(2), L151-L155.

Jeffers, S. V., Barnes, J. R. \& Collier Cameron, A. (2002) The latitude distribution of star-spots on He 699. MNRAS, 331(3), 666-672. 
Jeffers, S. V., Barnes, J. R., Jones, H. R. A. ET AL. (2014) Is it possible to detect planets around young active $\mathrm{G}$ and K dwarfs? MNRAS, 438(4), 2717-2731.

JefFers, S. V., Schöfer, P., LAmert, A. ET AL. (2018) CARMENES input catalogue of M dwarfs. III. Rotation and activity from high-resolution spectroscopic observations. A\&A, 614, A76.

Jenkins, J. S., Ramsey, L. W., Jones, H. R. A. et Al. (2009) Rotational Velocities for M Dwarfs. ApJ, 704(2), 975-988.

Johnson, E. N., Czesla, S., Fuhrmeister, B. et al. (2021) Simultaneous photometric and CARMENES spectroscopic monitoring of fast-rotating M dwarf GJ 3270. Discovery of a post-flare corotating feature. $A \mathcal{E} A, \mathbf{6 5 1}, \mathrm{A} 105$.

Johnson, H. L. \& Morgan, W. W. (1953) Fundamental stellar photometry for standards of spectral type on the Revised System of the Yerkes Spectral Atlas. ApJ, 117, 313.

Joy, A. H. (1947) Radial Velocities and Spectral Types of 181 Dwarf Stars. ApJ, 105, 96.

Joy, A. H. \& Авт, H. A. (1974) Spectral Types of M Dwarf Stars. ApJS, $28,1$.

Joy, A. H. \& Humason, M. L. (1949) Observations of the Faint Dwarf Star L 726-8. PASP, 61(360), 133134.

Judge, P. G., Carlsson, M. \& Stein, R. F. (2003) On the Origin of the Basal Emission from Stellar Atmospheres: Analysis of Solar C II Lines. ApJ, 597(2), 1158-1177.

Kafka, S. \& Honeycutt, R. K. (2006) Spectroscopy of Active and Inactive M Dwarfs in Praesepe. AJ, 132(4), 1517-1526.

KAHLER, S. W. (1992) Solar flares and coronal mass ejections. ARAEA, 30, 113-141.

Kasting, J. F., Whitmire, D. P. \& Reynolds, R. T. (1993) Habitable Zones around Main Sequence Stars. Icarus, 101(1), 108-128.

Kay, C., Opher, M. \& Kornbleuth, M. (2016) Probability of CME Impact on Exoplanets Orbiting M Dwarfs and Solar-like Stars. ApJ, 826(2), 195.

Kerber, F., Nave, G., Sansonetti, C. J., Bristow, P. \& Rosa, M. R. (2007) The Spectrum of ThAr Hollow Cathode Lamps in the 900-4500 nm Region: Establishing Wavelength Standards for the Calibration of VLT Spectrographs. In The Future of Photometric, Spectrophotometric and Polarimetric Standardization, edited by C. Sterken, vol. 364 of Astronomical Society of the Pacific Conference Series, pp. 461-478.

Khodachenko, M. L., Lammer, H., Lichtenegger, H. I. M. et AL. (2007) Mass loss of "Hot Jupiters"-Implications for CoRoT discoveries. Part I: The importance of magnetospheric protection of a planet against ion loss caused by coronal mass ejections. Planet. Space Sci., 55(5), 631-642.

KIRAga, M. (2012) ASAS Photometry of ROSAT Sources. I. Periodic Variable Stars Coincident with Bright Sources from the ROSAT All Sky Survey. Acta Astron., 62(1), 67-95.

Kiraga, M. \& Stepien, K. (2007) Age-Rotation-Activity Relations for M Dwarf Stars. Acta Astron., 57, $149-172$.

Kirkpatrick, J. D., Henry, T. J. \& McCarthy, Donald W., J. (1991) A Standard Stellar Spectral Sequence in the Red/Near-Infrared: Classes K5 to M9. ApJS, 77, 417. 
Kirkpatrick, J. D., Reid, I. N., Liebert, J. ET Al. (1999) Dwarfs Cooler than “M“: The Definition of Spectral Type "L" Using Discoveries from the 2 Micron All-Sky Survey (2MASS). ApJ, 519(2), 802-833.

Kopparapu, R. K., Ramirez, R., Kasting, J. F. et AL. (2013) Habitable Zones around Main-sequence Stars: New Estimates. ApJ, 765(2), 131.

Kotani, T., Tamura, M., Suto, H. ET al. (2014) Infrared Doppler instrument (IRD) for the Subaru telescope to search for Earth-like planets around nearby M-dwarfs. In Proc. SPIE, vol. 9147 of Society of PhotoOptical Instrumentation Engineers (SPIE) Conference Series, pp. 411-422.

Kowalski, A. F., Hawley, S. L., Hilton, E. J. et Al. (2009) M Dwarfs in Sloan Digital Sky Survey Stripe 82: Photometric Light Curves and Flare Rate Analysis. AJ, 138(2), 633-648.

Kowalski, A. F., Hawley, S. L., Holtzman, J. A., Wisniewski, J. P. \& Hilton, E. J. (2010) A White Light Megaflare on the dM4.5e Star YZ CMi. ApJL, 714(1), L98-L102.

Kron, G. E. (1947) The Probable Detecting of Surface Spots on AR Lacertae B. PASP, 59(350), 261.

Kuerster, M., Schmitt, J. H. M. M. \& Cutispoto, G. (1994) Doppler imaging with a CLEAN-like approach II. A photospheric image of AB Doradus (=HD 36705). AEEA, 289, 899-921.

KunKel, W. E. (1970) On the Spectra of Stellar Flares. ApJ, 161, 503.

Lacy, C. H., Moffett, T. J. \& Evans, D. S. (1976) UV Ceti stars: statistical analysis of observational data. ApJS, 30, 85-96.

Lafarga, M., Ribas, I., Lovis, C. ET al. (2020) The CARMENES search for exoplanets around M dwarfs. Radial velocities and activity indicators from cross-correlation functions with weighted binary masks. $A \mathcal{E} A$, 636, A36.

Lagrange, A. M., Desort, M. \& Meunier, N. (2010) Using the Sun to estimate Earth-like planets detection capabilities . I. Impact of cold spots. AE A, 512, A38.

Lammer, H., Selsis, F., Ribas, I. et al. (2003) Atmospheric Loss of Exoplanets Resulting from Stellar X-Ray and Extreme-Ultraviolet Heating. ApJL, 598(2), L121-L124.

Lamy, P. L., Floyd, O., Boclet, B. et AL. (2019) Coronal Mass Ejections over Solar Cycles 23 and 24. Space Sci. Rev., 215(5), 39.

Laughlin, G., Bodenheimer, P. \& Adams, F. C. (1997) The End of the Main Sequence. ApJ, 482(1), 420-432.

Leighton, R. B. (1964) Transport of Magnetic Fields on the Sun. ApJ, 140, 1547.

Leighton, R. B. (1969) A Magneto-Kinematic Model of the Solar Cycle. ApJ, 156, 1.

Leitzinger, M., Odert, P., Ribas, I. ET AL. (2011) Search for indications of stellar mass ejections using FUV spectra. A\&A, 536, A62.

Lépine, S., Hilton, E. J., Mann, A. W. ET AL. (2013) A Spectroscopic Catalog of the Brightest $(\mathrm{J}<9)$ M Dwarfs in the Northern Sky. AJ, 145(4), 102.

Lépine, S., Rich, R. M. \& Shara, M. M. (2003) Spectroscopy of New High Proper Motion Stars in the Northern Sky. I. New Nearby Stars, New High-Velocity Stars, and an Enhanced Classification Scheme for M Dwarfs. AJ, 125(3), 1598-1622. 
Lépine, S., Rich, R. M. \& Shara, M. M. (2007) Revised Metallicity Classes for Low-Mass Stars: Dwarfs (dM), Subdwarfs (sdM), Extreme Subdwarfs (esdM), and Ultrasubdwarfs (usdM). ApJ, 669(2), 12351247.

Liebert, J., Kirkpatrick, J. D., Reid, I. N. \& Fisher, M. D. (1999) A 2MASS Ultracool M Dwarf Observed in a Spectacular Flare. ApJ, 519(1), 345-353.

Lindegren, L. \& Dravins, D. (2003) The fundamental definition of “radial velocity”. AE A, 401, 1185-1201.

Lingam, M. \& Loeb, A. (2017a) Reduced Diversity of Life around Proxima Centauri and TRAPPIST-1. $A p J L, \mathbf{8 4 6}(2), \mathrm{L} 21$.

Lingam, M. \& Loeb, A. (2017b) Risks for Life on Habitable Planets from Superflares of Their Host Stars. $A p J, \mathbf{8 4 8}(1), 41$.

Linsky, J. L. (2000) Stellar flares: How common? How Important? In Cosmic Explosions: Tenth AstroPhysics Conference, edited by S. S. Holt \& W. W. Zhang, vol. 522 of American Institute of Physics Conference Series, pp. 389-399.

Linsky, J. L. (2017) Stellar Model Chromospheres and Spectroscopic Diagnostics. ARAEA, 55(1), 159-211.

Linsky, J. L. \& Avrett, E. H. (1970) The Solar H and K Lines. PASP, 82(485), 169.

Linsky, J. L., Hunten, D. M., Sowell, R., Glackin, D. L. \& Kelch, W. L. (1979) Stellar model chromospheres. XI. A survey of CA II $\lambda 8542$ line profiles in late-type stars of differing chromospheric activity. ApJS, 41, 481-500.

Linsky, J. L. \& Schöller, M. (2015) Observations of Strong Magnetic Fields in Nondegenerate Stars. Space Sci. Rev., 191(1-4), 27-76.

Littmann, M., Espenak, F. \& Willcox, K. (2008) Totality: Eclipses of the Sun. 3rd edn., Oxford University Press.

Livingston, W., Wallace, L., White, O. R. \& Giampapa, M. S. (2007) Sun-as-a-Star Spectrum Variations 1974-2006. ApJ, 657(2), 1137-1149.

Lockyer, J. N. (1868) Spectroscopic Observation of the Sun, No. II. Proceedings of the Royal Society of London Series I, 17, 131-132.

Loewenstein, E. V. (1966) The history and current status of Fourier transform spectroscopy. Appl. Opt., $\mathbf{5}(5), 845$

Lovis, C., Dumusque, X., Santos, N. C. ET AL. (2011) The HARPS search for southern extra-solar planets. XXXI. Magnetic activity cycles in solar-type stars: statistics and impact on precise radial velocities. arXiv e-prints, arXiv:1107.5325.

Luger, R. \& Barnes, R. (2015) Extreme Water Loss and Abiotic O2Buildup on Planets Throughout the Habitable Zones of M Dwarfs. Astrobiology, 15(2), 119-143.

Luger, R., Barnes, R., Lopez, E. ET AL. (2015) Habitable Evaporated Cores: Transforming Mini-Neptunes into Super-Earths in the Habitable Zones of M Dwarfs. Astrobiology, 15(1), 57-88.

Luque, R., Nowak, G., Pallé, E. et al. (2019a) Detection and characterization of an ultra-dense subNeptunian planet orbiting the Sun-like star K2-292. AEA, 623, A114. 
Luque, R., Pallé, E., Kossakowski, D. et Al. (2019b) Planetary system around the nearby M dwarf GJ 357 including a transiting, hot, Earth-sized planet optimal for atmospheric characterization. A\&A, 628, A39.

Mahadevan, S., Ramsey, L. W., Terrien, R. et Al. (2014) The Habitable-zone Planet Finder: A status update on the development of a stabilized fiber-fed near-infrared spectrograph for the for the HobbyEberly telescope. In Proc. SPIE, vol. 9147 of Society of Photo-Optical Instrumentation Engineers (SPIE) Conference Series, pp. 543-552.

Mallik, S. V. (1997) The CA II triplet lines as diagnostics of luminosity, metallicity and chromospheric activity in cool stars. A\&AS, 124, 359-384.

Marcy, G. W. \& Butler, R. P. (1992) Precision Radial Velocities with an Iodine Absorption cell. PASP, $104,270$.

MarisKa, J. T. (1986) The quiet solar transition region. ARAEA, 24, 23-48.

Marsden, S. C., Petit, P., Jeffers, S. V. et al. (2014) A BCool magnetic snapshot survey of solar-type stars. MNRAS, 444(4), 3517-3536.

Martín, E. L., Delfosse, X., Basri, G. et al. (1999) Spectroscopic Classification of Late-M and L Field Dwarfs. $A J, \mathbf{1 1 8}(5), 2466-2482$.

Martin, J., Fuhrmeister, B., Mittag, M. et AL. (2017) The Ca II infrared triplet's performance as an activity indicator compared to $\mathrm{Ca}$ II $\mathrm{H}$ and $\mathrm{K}$. Empirical relations to convert $\mathrm{Ca}$ II infrared triplet measurements to common activity indices. $A \mathcal{E} A, \mathbf{6 0 5}, \mathrm{A} 113$.

Martínez-Arnáiz, R., López-Santiago, J., Crespo-Chacón, I. \& Montes, D. (2011) Effect of magnetic activity saturation in chromospheric flux-flux relationships. MNRAS, 414(3), 2629-2641.

Martínez-Rodríguez, H. (2014) CARMENES target characterisation: mining public archives for highresolution spectra of $M d$ warfs with exoplanets. Master's thesis, Universidad Complutense de Madrid, Spain.

Mauas, P. J. D. (2000) Building Reliable Models of M Dwarf Chromospheres: The Spectral Diagnostics. $A p J, \mathbf{5 3 9}(2), 858-864$.

Maunder, E. W. (1922) The Prolonged Sunspot Minimum, 1645-1715. Journal of the British Astronomical Association, 32, 140-145.

Mayor, M., PePe, F., Queloz, D. Et Al. (2003) Setting New Standards with HARPS. The Messenger, 114, 20-24.

Mayor, M. \& Queloz, D. (1995) A Jupiter-mass companion to a solar-type star. Nature, 378(6555), 355359.

McLean, M., Berger, E. \& Reiners, A. (2012) The Radio Activity-Rotation Relation of Ultracool Dwarfs. ApJ, 746(1), 23.

Messina, S., Desidera, S., Lanzafame, A. C., Turatto, M. \& Guinan, E. F. (2011) RaCE-OC project: rotation and variability in the $\in$ Chamaeleontis, Octans, and Argus stellar associations. AEA, 532, A10.

Metcalf, A. J., Anderson, T., Bender, C. F. et Al. (2019) Stellar spectroscopy in the near-infrared with a laser frequency comb. Optica, 6(2), 233.

Meunier, N., Lagrange, A. M. \& Desort, M. (2010) Reconstructing the solar integrated radial velocity using MDI/SOHO. AEA, 519, A66. 
Montes, D., Fernandez-Figueroa, M. J., de Castro, E. \& Cornide, M. (1995) Excess H $\alpha$ emission in chromospherically active binaries. AEA, 294, 165-176.

Morales, J. C., Mustill, A. J., Ribas, I. Et Al. (2019) A giant exoplanet orbiting a very-low-mass star challenges planet formation models. Science, 365(6460), 1441-1445.

Morgan, W. W., KeEnan, P. C. \& Kellman, E. (1943) An atlas of stellar spectra, with an outline of spectral classification. University of Chicago Press.

Morin, J., Donati, J. F., Petit, P. et al. (2008) Large-scale magnetic topologies of mid M dwarfs. MNRAS, $390(2), 567-581$.

Morin, J., Donati, J. F., Petit, P. et Al. (2010) Large-scale magnetic topologies of late M dwarfs. MNRAS, 407(4), 2269-2286.

Morris, B. M., Agol, E., Davenport, J. R. A. \& Hawley, S. L. (2018) Possible Bright Starspots on TRAPPIST-1. ApJ, 857(1), 39.

Moschou, S.-P., Drake, J. J., Cohen, O., Alvarado-Gomez, J. D. \& Garraffo, C. (2017) A Monster CME Obscuring a Demon Star Flare. ApJ, 850(2), 191.

Moschou, S.-P., Drake, J. J., Cohen, O. et al. (2019) The Stellar CME-Flare Relation: What Do Historic Observations Reveal? ApJ, 877(2), 105.

Muheki, P., Guenther, E. W., Mutabazi, T. \& Jurua, E. (2020) High-resolution spectroscopy of flares and CMEs on AD Leonis. AEA, 637, A13.

Mulders, G. D., Pascucci, I. \& ApaI, D. (2015) A Stellar-mass-dependent Drop in Planet Occurrence Rates. $A p J, \mathbf{7 9 8}(2), 112$.

Mullan, D. J. \& Paudel, R. R. (2019) Origin of Radio-quiet Coronal Mass Ejections in Flare Stars. ApJ, $\mathbf{8 7 3}(1), 1$

Murphy, M. T., Udem, T., Holzwarth, R. ET Al. (2007) High-precision wavelength calibration of astronomical spectrographs with laser frequency combs. MNRAS, 380(2), 839-847.

Nagel, E., Czesla, S., Kaminski, A. ET Al. (submitted) The CARMENES search for exoplanets around M dwarfs. The template division telluric modeling technique and its application to optical and near-infrared radial velocities. $A \mathcal{E} A$, submitted.

Namekata, K., Maehara, H., Notsu, Y. et al. (2019) Lifetimes and Emergence/Decay Rates of Star Spots on Solar-type Stars Estimated by Kepler Data in Comparison with Those of Sunspots. ApJ, 871(2), 187.

Narain, U. \& Ulmschneider, P. (1990) Chromospheric and Coronal Heating Mechanisms. Space Sci. Rev., 54(3-4), 377-445.

Narain, U. \& Ulmschneider, P. (1996) Chromospheric and Coronal Heating Mechanisms II. Space Sci. Rev., 75(3-4), 453-509.

Neff, J. E., O’Neal, D. \& SAAR, S. H. (1995) Absolute Measurements of Starspot Area and Temperature: II Pegasi in 1989 October. ApJ, 452, 879.

Newton, E. R., Charbonneau, D., Irwin, J. et al. (2014) Near-infrared Metallicities, Radial Velocities, and Spectral Types for 447 Nearby M Dwarfs. AJ, 147(1), 20. 
Newton, E. R., Irwin, J., Charbonneau, D. et Al. (2016) The Rotation and Galactic Kinematics of Mid M Dwarfs in the Solar Neighborhood. ApJ, 821(2), 93.

Newton, E. R., Irwin, J., Charbonneau, D. ET Al. (2017) The H $\alpha$ Emission of Nearby M Dwarfs and its Relation to Stellar Rotation. ApJ, 834(1), 85.

Niven, C. (1874) On a method of finding the parallax of double stars and on the displacement of the lines in the spectrum of a planet. MNRAS, 34, 339-347.

Nortmann, L., Pallé, E., Salz, M. et Al. (2018) Ground-based detection of an extended helium atmosphere in the Saturn-mass exoplanet WASP-69b. Science, 362(6421), 1388-1391.

Noyes, R. W., Hartmann, L. W., Baliunas, S. L., Duncan, D. K. \& Vaughan, A. H. (1984) Rotation, convection, and magnetic activity in lower main-sequence stars. ApJ, 279, 763-777.

O’Neal, D., Neff, J. E. \& SaAr, S. H. (1998) Measurements of Starspot Parameters on Active Stars using Molecular Bands in Echelle Spectra. ApJ, 507(2), 919-937.

OssendriJver, M. (2003) The solar dynamo. A $\mathcal{E} A$ Rev., 11(4), 287-367.

Osten, R. A., Hawley, S. L., Allred, J. C., Johns-Krull, C. M. \& Roark, C. (2005) From Radio to X-Ray: Flares on the dMe Flare Star EV Lacertae. ApJ, 621(1), 398-416.

Owen, J. E. \& Mohanty, S. (2016) Habitability of terrestrial-mass planets in the HZ of M Dwarfs - I. $\mathrm{H} /$ He-dominated atmospheres. MNRAS, 459(4), 4088-4108.

Pallavicini, R., Golub, L., Rosner, R. et Al. (1981) Relations among stellar X-ray emission observed from Einstein, stellar rotation and bolometric luminosity. ApJ, 248, 279-290.

Palle, E., Nowak, G., Luque, R. et al. (2019) Detection and Doppler monitoring of K2-285 (EPIC 246471491), a system of four transiting planets smaller than Neptune. A\&A, 623, A41.

PARKer, E. N. (1955) Hydromagnetic Dynamo Models. ApJ, 122, 293.

PARKer, E. N. (1958) Dynamics of the Interplanetary Gas and Magnetic Fields. ApJ, 128, 664.

Parker, E. N. (1988) Nanoflares and the Solar X-Ray Corona. ApJ, 330, 474.

Parks, J. R., White, R. J., Baron, F. et al. (2015) First Images of Cool Starspots on a Star Other than the Sun: Interferometric Imaging of $\lambda$ Andromedae. arXiv e-prints, arXiv:1508.04755.

Passegger, V. M., Reiners, A., Jeffers, S. V. et Al. (2018) The CARMENES search for exoplanets around M dwarfs. Photospheric parameters of target stars from high-resolution spectroscopy. AEA, 615, A6.

Pecaut, M. J. \& Mamajek, E. E. (2013) Intrinsic Colors, Temperatures, and Bolometric Corrections of Pre-main-sequence Stars. ApJS, 208(1), 9.

Pepe, F., Cristiani, S., Rebolo, R. et al. (2021) ESPRESSO at VLT. On-sky performance and first results. $A \mathcal{E} A, \mathbf{6 4 5}, \mathrm{A} 96$.

Perryman, M. (2018) The Exoplanet Handbook. 2nd edn., Cambridge University Press.

Pestalozzi, M. R., Benz, A. O., Conway, J. E. \& Güdel, M. (2000) VlBI observations of two single dMe stars: spatial resolution and astrometry. $A \mathcal{E} A, 353,569-574$.

Petrovay, K. \& van Driel-Gesztelyi, L. (1997) Making Sense of Sunspot Decay. I. Parabolic Decay Law and Gnevyshev-Waldmeier Relation. Sol. Phys., 176(2), 249-266. 
Pettersen, B. R. (1989) A Review of Stellar Flares and Their Characteristics. Sol. Phys., 121(1-2), $299-312$.

Pevtsov, A. A., Fisher, G. H., Acton, L. W. et Al. (2003) The Relationship Between X-Ray Radiance and Magnetic Flux. ApJ, 598(2), 1387-1391.

Piskunov, N. E. \& Valenti, J. A. (2002) New algorithms for reducing cross-dispersed echelle spectra. $A \mathcal{E} A$, 385, 1095-1106.

Pizzolato, N., Maggio, A., Micela, G., Sciortino, S. \& Ventura, P. (2003) The stellar activity-rotation relationship revisited: Dependence of saturated and non-saturated X-ray emission regimes on stellar mass for late-type dwarfs. A\& $A, 397,147-157$.

Poppenhaeger, K. (2019) How stars and planets interact: A look through the high-energy window. Astronomische Nachrichten, 340(4), 329-333.

Preston, G. W. (1971) The Mean Surface Fields of Magnetic Stars. ApJ, 164, 309.

Queloz, D., Henry, G. W., Sivan, J. P. et Al. (2001) No planet for HD 166435. AEA A, 379, 279-287.

Quirrenbach, A., Amado, P. J., Ribas, I. et Al. (2018) CARMENES: high-resolution spectra and precise radial velocities in the red and infrared. In Proc. SPIE, vol. 10702 of Society of Photo-Optical Instrumentation Engineers (SPIE) Conference Series, pp. 246-263.

Radick, R. R., Lockwood, G. W., Skiff, B. A. \& Baliunas, S. L. (1998) Patterns of Variation among Sun-like Stars. ApJS, 118(1), 239-258.

ReID, I. N. \& Hawley, S. L. (2005) New light on dark stars : red dwarfs, low-mass stars, brown dwarfs. 2nd edn., Praxis Publishing Ltd.

Reid, I. N., Hawley, S. L. \& Gizis, J. E. (1995) The Palomar/MSU Nearby-Star Spectroscopic Survey. I. The Northern M Dwarfs -Bandstrengths and Kinematics. AJ, 110, 1838.

ReIners, A. (2012) Observations of Cool-Star Magnetic Fields. Living Reviews in Solar Physics, 9(1), 1.

Reiners, A. \& Basri, G. (2008) Chromospheric Activity, Rotation, and Rotational Braking in M and L Dwarfs. ApJ, 684(2), 1390-1403.

Reiners, A. \& BAsri, G. (2010) A Volume-Limited Sample of 63 M7-M9.5 Dwarfs. II. Activity, Magnetism, and the Fade of the Rotation-Dominated Dynamo. ApJ, 710(2), 924-935.

Reiners, A., Basri, G. \& Browning, M. (2009) Evidence for Magnetic Flux Saturation in Rapidly Rotating M Stars. ApJ, 692(1), 538-545.

Reiners, A., Joshi, N. \& Goldman, B. (2012) A Catalog of Rotation and Activity in Early-M Stars. AJ, 143(4), 93.

Reiners, A., Ribas, I., Zechmeister, M. ET AL. (2018a) The CARMENES search for exoplanets around M dwarfs. HD147379 b: A nearby Neptune in the temperate zone of an early-M dwarf. A\&A, 609, L5.

Reiners, A. \& Schmitt, J. H. M. M. (2003) Rotation and differential rotation in field F- and G-type stars. $A \mathcal{E} A$, 398, 647-661.

Reiners, A., Schüssler, M. \& Passegger, V. M. (2014) Generalized Investigation of the Rotation-Activity Relation: Favoring Rotation Period instead of Rossby Number. ApJ, 794(2), 144.

Reiners, A. \& Zechmeister, M. (2020) Radial Velocity Photon Limits for the Dwarf Stars of Spectral Classes FM. ApJS, 247(1), 11. 
Reiners, A., Zechmeister, M., Caballero, J. A. et Al. (2018b) The CARMENES search for exoplanets around M dwarfs. High-resolution optical and near-infrared spectroscopy of 324 survey stars. AEA, 612 , A49.

Reinhold, T., Reiners, A. \& Basri, G. (2013) Rotation and differential rotation of active Kepler stars. AEA, 560, A4.

Riaz, B., Gizis, J. E. \& Harvin, J. (2006) Identification of New M Dwarfs in the Solar Neighborhood. AJ, 132(2), 866-872.

Ribas, I., Guinan, E. F., Güdel, M. \& Audard, M. (2005) Evolution of the Solar Activity over Time and Effects on Planetary Atmospheres. I. High-Energy Irradiances (1-1700 Å). ApJ, 622(1), 680-694.

Ribas, I., Tuomi, M., Reiners, A. et AL. (2018) A candidate super-Earth planet orbiting near the snow line of Barnard's star. Nature, 563(7731), 365-368.

Robertson, P., Bender, C., Mahadevan, S., Roy, A. \& Ramsey, L. W. (2016) Proxima Centauri as a Benchmark for Stellar Activity Indicators in the Near-infrared. ApJ, 832(2), 112.

Robertson, P., Roy, A. \& Mahadevan, S. (2015) Stellar Activity Mimics a Habitable-zone Planet around Kapteyn's Star. ApJL, 805(2), L22.

Robinson, JR., R. D. (1980) Magnetic field measurements on stellar sources - A new method. ApJ, 239, 961-967.

Roettenbacher, R. M., Monnier, J. D., Korhonen, H. et Al. (2016) No Sun-like dynamo on the active star $\zeta$ Andromedae from starspot asymmetry. Nature, 533(7602), 217-220.

Russell, H. N. (1913) “Giant” and “dwarf” stars. The Observatory, 36, 324-329.

SAAR, S. H. (1988) Measurements of Magnetic Fields on Cool Stars. In The Impact of Very High S/N Spectroscopy on Stellar Physics, edited by G. Cayrel de Strobel \& M. Spite, vol. 132 of IAU Symposium, pp. 295-300.

SaAr, S. H. \& Donahue, R. A. (1997) Activity-Related Radial Velocity Variation in Cool Stars. ApJ, 485(1), 319-327.

Sanz-Forcada, J. \& Dupree, A. K. (2008) Active cool stars and He I 10830 A: the coronal connection. $A \mathcal{E} A, \mathbf{4 8 8}(2), 715-721$.

Sanz-Forcada, J., Micela, G., Ribas, I. et al. (2011) Estimation of the XUV radiation onto close planets and their evaporation. $A \mathcal{E} A, \mathbf{5 3 2}, \mathrm{A} 6$.

Sarmiento, L. F., Reiners, A., Huke, P. et Al. (2018) Comparing the emission spectra of U and Th hollow cathode lamps and a new $\mathrm{U}$ line list. $A \mathcal{E} A, \mathbf{6 1 8}, \mathrm{A} 118$.

Schäfer, S., Guenther, E. W., Reiners, A. et al. (2018) Two Fabry-Pérots and two calibration units for CARMENES. In Proc. SPIE, vol. 10702 of Society of Photo-Optical Instrumentation Engineers (SPIE) Conference Series, pp. 2141-2150.

Schatzman, E. (1949) The heating of the solar corona and chromosphere. Annales d'Astrophysique, $12,203$.

Schlieder, J. E., Lépine, S., Rice, E. et AL. (2012) The Na 8200 Å Doublet as an Age Indicator in Low-mass Stars. AJ, 143(5), 114. 
Schmidt, S. J., Cruz, K. L., Bongionno, B. J., Liebert, J. \& Reid, I. N. (2007) Activity and Kinematics of Ultracool Dwarfs, Including an Amazing Flare Observation. AJ, 133(5), 2258-2273.

Schmidt, S. J., Kowalski, A. F., Hawley, S. L. Et Al. (2012) Probing the Flare Atmospheres of M Dwarfs Using Infrared Emission Lines. ApJ, 745(1), 14.

Schmidt, S. J., Shappee, B. J., van Saders, J. L. et al. (2019) The Largest M Dwarf Flares from ASAS-SN. $A p J, \mathbf{8 7 6}(2), 115$.

Schöfer, P., Jeffers, S. V., Reiners, A. ET AL. (2019) The CARMENES search for exoplanets around M dwarfs. Activity indicators at visible and near-infrared wavelengths. A\&A, 623, A44.

Schöfer, P., JefFers, S. V., ReINERs, A. ET AL. (submitted) The CARMENES search for exoplanets around M dwarfs. Rotational variation in activity indicators of EV Lac, YZ CMi, and two northern slow rotators. $A \mathcal{E} A$, submitted.

Scholz, R. D., Meusinger, H. \& JAhreiss, H. (2005) Search for nearby stars among proper motion stars selected by optical-to-infrared photometry. III. Spectroscopic distances of 322 NLTT stars. AE $A, \mathbf{4 4 2}(1)$, 211-227.

SchriJver, C. J. (1987) Magnetic structure in cool stars. XI. Relations between radiative fluxes mesuring stellar activity, and evidence for two components in stellar chromospheres. AEAA, 172, 111-123.

SchriJver, C. J., Beer, J., Baltensperger, U. et al. (2012) Estimating the frequency of extremely energetic solar events, based on solar, stellar, lunar, and terrestrial records. Journal of Geophysical Research (Space Physics), 117(A8), A08103.

SchriJver, C. J. \& ZwaAn, C. (1991) Activity in tidally interacting binaries. AEAA, 251, 183.

Schwabe, H. (1844) Sonnenbeobachtungen im Jahre 1843. Astronomische Nachrichten, 21(15), 233.

Schwarzschild, M. (1948) On Noise Arising from the Solar Granulation. ApJ, 107, 1.

Schweitzer, A., Passegger, V. M., Cifuentes, C. et Al. (2019) The CARMENES search for exoplanets around $\mathrm{M}$ dwarfs. Different roads to radii and masses of the target stars. A\&A, 625, A68.

Segura, A., Walkowicz, L. M., Meadows, V., Kasting, J. \& Hawley, S. (2010) The Effect of a Strong Stellar Flare on the Atmospheric Chemistry of an Earth-like Planet Orbiting an M Dwarf. Astrobiology, 10(7), 751-771.

SeIfahrt, A., KäUfl, H. U., ZäNGl, G. eT Al. (2010) Synthesising, using, and correcting for telluric features in high-resolution astronomical spectra . A near-infrared case study using CRIRES. A\&A, 524, A11.

SEMEL, M. (1989) Zeeman-Doppler imaging of active stars. I - Basic principles. AEFA, 225, 456-466.

Shapiro, A. I., Solanki, S. K., Krivova, N. A., Yeo, K. L. \& Schmutz, W. K. (2016) Are solar brightness variations faculae- or spot-dominated? $A \mathcal{E} A, \mathbf{5 8 9}, \mathrm{A} 46$.

Shine, R. A. \& Linsky, J. L. (1972) Physical Properties of Solar Chromospheric Plages. I. Line Profiles of the Ca II H, K, and Infrared Triplet Lines. Sol. Phys., 25(2), 357-379.

Shrolnik, E., Bohlender, D. A., Walker, G. A. H. \& Collier Cameron, A. (2008) The On/Off Nature of Star-Planet Interactions. ApJ, 676(1), 628-638.

Shrolnik, E., Liu, M. C. \& Reid, I. N. (2009) Identifying the Young Low-mass Stars within 25 pc. I. Spectroscopic Observations. ApJ, 699(1), 649-666. 
Shrolnik, E., Walker, G. A. H., Bohlender, D. A., Gu, P. G. \& Kürster, M. (2005) Hot Jupiters and Hot Spots: The Short- and Long-Term Chromospheric Activity on Stars with Giant Planets. ApJ, 622(2), 1075-1090.

Shopov, Y. Y., Stoykova, D. A., Stortchkova, K. et AL. (2008) Structure of the solar dust corona and its interaction with the other coronal components. Journal of Atmospheric and Solar-Terrestrial Physics, 70(2-4), 356-364.

Short, C. I. \& Doyle, J. G. (1997) Chromospheric line blanketing and the hydrogen spectrum in M dwarfs. $A \mathcal{E} A$, 326, 287-299.

Short, C. I. \& Doyle, J. G. (1998) Pa-beta as a chromospheric diagnostic in M dwarfs. AEA, 331, L5-L8.

Shulyak, D., Reiners, A., Engeln, A. et al. (2017) Strong dipole magnetic fields in fast rotating fully convective stars. Nature Astronomy, 1, 0184

Shulyak, D., Reiners, A., Nagel, E. ET al. (2019) Magnetic fields in M dwarfs from the CARMENES survey. A\&A, 626, A86.

Siarkowski, M., Pres, P., Drake, S. A., White, N. E. \& Singh, K. P. (1996) Corona(e) of AR Lacertae. II. The Spatial Structure. ApJ, 473, 470.

SiLva, A. V. R. (2003) Method for Spot Detection on Solar-like Stars. ApJL, 585(2), L147-L150.

Silva-Valio, A., Lanza, A. F., Alonso, R. \& Barge, P. (2010) Properties of starspots on CoRoT-2. AEA, 510, A25.

Simon, T., Ayres, T. R., Redfield, S. \& Linsky, J. L. (2002) Limits on Chromospheres and Convection among the Main-Sequence A Stars. ApJ, 579(2), 800-809.

Skumanich, A. (1972) Time Scales for Ca II Emission Decay, Rotational Braking, and Lithium Depletion. ApJ, 171, 565.

Smith, K., Güdel, M. \& Audard, M. (2005) Flares observed with XMM-Newton and the VLA. AEAA, 436(1), 241-251.

Soderblom, D. R., Stauffer, J. R., Hudon, J. D. \& Jones, B. F. (1993) Rotation and Chromospheric Emission among $\mathrm{F}, \mathrm{G}$, and $\mathrm{K}$ Dwarfs of the Pleiades. ApJS, 85, 315.

Spörer, F. G. W. (1887) Über die Periodizität der Sonnenflecken seit dem Jahre 1618, vornehmlich in Bezug auf die heliographische Breite derselben, und Hinweis auf eine erhebliche Streuung dieser Periodizität während eines langen Zeitraumes. Vierteljahrsschrift der Astronomischen Gesellschaft, 22, 323-329.

Spruit, H. C. (2011) Theories of the Solar Cycle: A Critical View. In The Sun, the Solar Wind, and the Heliosphere, edited by M. P. Miralles \& J. Sánchez Almeida, vol. 4, p. 39.

Stassun, K. G., Hebb, L., Covey, K. et al. (2011) The M4 Transition: Toward a Comprehensive Understanding of the Transition into the Fully Convective Regime. In $16^{\text {th }}$ Cambridge Workshop on Cool Stars, Stellar Systems, and the Sun, edited by C. Johns-Krull, M. K. Browning \& A. A. West, vol. 448 of Astronomical Society of the Pacific Conference Series, pp. 505-516.

Stauffer, J. R. \& Hartmann, L. W. (1986) Chromospheric Activity, Kinematics, and Metallicities of Nearby M Dwarfs. ApJS, 61, 531.

Stelzer, B., Marino, A., Micela, G., López-Santiago, J. \& Liefke, C. (2013) The UV and X-ray activity of the M dwarfs within $10 \mathrm{pc}$ of the Sun. MNRAS, 431(3), 2063-2079. 
Stewart, R. T., Innis, J. L., Slee, O. B., Nelson, G. J. \& Wright, A. E. (1988) A Relation Between Radio Luminosity and Rotation for Late-Type Stars. AJ, 96, 371.

Stix, M. (1989) The Sun's Differential Rotation. Reviews in Modern Astronomy, 2, 248-266.

Strassmeier, K. G. (2009) Starspots. A\&A Rev., 17(3), 251-308.

Strassmeier, K. G., Bartus, J., Cutispoto, G. \& Rodono, M. (1997) Starspot photometry with robotic telescopes: Continuous UBV and $\mathrm{V}(\mathrm{RI})_{\mathrm{C}}$ photometry of 23 stars in 1991-1996. A\&AS, 125, 11-63.

Strassmeier, K. G., Rice, J. B., Wehlau, W. H. et al. (1991) Doppler imaging of hig-latitude SPOT activity on HD 26337. AE्EA, 247, 130.

Suárez Mascareño, A., Rebolo, R. \& González Hernández, J. I. (2016) Magnetic cycles and rotation periods of late-type stars from photometric time series. $A \mathcal{E} A, \mathbf{5 9 5}, \mathrm{A} 12$.

Suárez Mascareño, A., Rebolo, R., González Hernández, J. I. \& Esposito, M. (2015) Rotation periods of late-type dwarf stars from time series high-resolution spectroscopy of chromospheric indicators. MNRAS, 452(3), 2745-2756.

Suárez Mascareño, A., Rebolo, R., González Hernández, J. I. \& Esposito, M. (2017) Characterization of the radial velocity signal induced by rotation in late-type dwarfs. MNRAS, 468(4), 4772-4781.

Suárez Mascareño, A., Rebolo, R., González Hernández, J. I. et al. (2018) HADES RV programme with HARPS-N at TNG. VII. Rotation and activity of M-dwarfs from time-series high-resolution spectroscopy of chromospheric indicators. AEA, 612, A89.

TAl-Or, L., Trifonov, T., Zucker, S., Mazeh, T. \& Zechmeister, M. (2019) Correcting HIRES/Keck radial velocities for small systematic errors. MNRAS, 484(1), L8-L13.

TAl-Or, L., Zechmeister, M., Reiners, A. ET Al. (2018) The CARMENES search for exoplanets around M dwarfs. Radial-velocity variations of active stars in visual-channel spectra. A\&A, 614, A122.

Tian, F., France, K., Linsky, J. L., Mauas, P. J. D. \& Vieytes, M. C. (2014) High stellar FUV/NUV ratio and oxygen contents in the atmospheres of potentially habitable planets. Earth and Planetary Science Letters, 385, 22-27.

Tinney, C. G. \& REID, I. N. (1998) High-resolution spectra of very low-mass stars. MNRAS, 301(4), 10311048.

Torres, C. A. O., Quast, G. R., Da Silva, L. ET AL. (2006) Search for associations containing young stars (SACY). I. Sample and searching method. AEA, 460(3), 695-708.

Toupance, G., Bossard, A. \& Raulin, F. (1977) Far UV irradiation of model prebiotic atmospheres. Origins of Life, 8(3), 259-266.

Tousey, R. (1973) The solar corona. In Proceedings of Open Meetings of Working Groups on Physical Sciences of the 15th Plenary Meeting of COSPAR, Madrid, Spain, 10-24 May, 1972, edited by M. J. Rycroft \& S. K. Runcorn, vol. 2 of Space Research XIII, pp. 713-730.

Tregloan-Reed, J. \& Unda-Sanzana, E. (2019) Simulations of starspot anomalies within TESS exoplanetary transit light curves. I. Detection limits of starspot anomalies in TESS light curves. AE A, 630, A114.

Trifonov, T., Kürster, M., Zechmeister, M. et AL. (2018) The CARMENES search for exoplanets around $\mathrm{M}$ dwarfs . First visual-channel radial-velocity measurements and orbital parameter updates of seven M-dwarf planetary systems. A\&A, 609, A117. 
Tsuj, T., Oнnaka, K. \& Aoki, W. (1996) Dust formation in stellar photospheres: a case of very low mass stars and a possible resolution on the effective temperature scale of $\mathrm{M}$ dwarfs. $A \mathcal{E} A, \mathbf{3 0 5}, \mathrm{L} 1$.

Tuomi, M., Anglada-Escudé, G., Gerlach, E. et AL. (2013) Habitable-zone super-Earth candidate in a sixplanet system around the K2.5V star HD 40307. A $\mathcal{E} A, \mathbf{5 4 9}$, A48.

Valenti, J. A. \& Johns-Krull, C. (2001) Magnetic Field Measurements for Cool Stars. In Magnetic Fields Across the Hertzsprung-Russell Diagram, edited by G. Mathys, S. K. Solanki \& D. T. Wickramasinghe, vol. 248 of Astronomical Society of the Pacific Conference Series, pp. 179-188.

Valenti, J. A., Piskunov, N. \& Johns-Krull, C. M. (1998) Spectral Synthesis of TiO Lines. ApJ, 498(2), $851-862$.

VAQUERo, J. M. (2007) Letter to the Editor: Sunspot observations by Theophrastus revisited. Journal of the British Astronomical Association, 117, 346.

Vaughan, A. H., Baliunas, S. L., Middelkoop, F. et al. (1981) Stellar rotation in lower main-sequence stars measured from time variations in $\mathrm{H}$ and $\mathrm{K}$ emission-line fluxes. I. Initial results. ApJ, 250, 276-283.

Vaughan, A. H., Preston, G. W. \& Wilson, O. C. (1978) Flux measurements of Ca II and K emission. PASP, 90, 267-274.

Vaughan, Arthur H., J. \& Zirin, H. (1968) The Helium Line $\lambda 10830$ A in Late-Type Stars. ApJ, $152,123$.

Vernazza, J. E., Avrett, E. H. \& Loeser, R. (1981) Structure of the solar chromosphere. III. Models of the EUV brightness components of the quiet sun. ApJS, 45, 635-725.

VIDA, K., Kriskovics, L., OLÁH, K. ET AL. (2016) Investigating magnetic activity in very stable stellar magnetic fields. Long-term photometric and spectroscopic study of the fully convective M4 dwarf V374 Pegasi. A\&A, 590, A11.

VidA, K., Leitzinger, M., Kriskovics, L. et AL. (2019) The quest for stellar coronal mass ejections in latetype stars. I. Investigating Balmer-line asymmetries of single stars in Virtual Observatory data. $A \mathcal{E} A$, 623, A49.

Vidal-Madjar, A., Lecavelier des Etangs, A., Désert, J. M. et Al. (2003) An extended upper atmosphere around the extrasolar planet HD209458b. Nature, 422(6928), 143-146.

Vidotto, A. A., Gregory, S. G., Jardine, M. et AL. (2014) Stellar magnetism: empirical trends with age and rotation. MNRAS, 441(3), 2361-2374.

Villadsen, J. \& Hallinan, G. (2019) Ultra-wideband Detection of 22 Coherent Radio Bursts on M Dwarfs. ApJ, 871(2), 214.

Vogt, S. S. (1979) A spectroscopic and photometric study of the star spot on HD 224085. PASP, $91,616$.

Vogt, S. S., Allen, S. L., Bigelow, B. C. ET Al. (1994) HIRES: the high-resolution echelle spectrometer on the Keck 10-m Telescope. In Proc. SPIE, edited by D. L. Crawford \& E. R. Craine, vol. 2198 of Society of Photo-Optical Instrumentation Engineers (SPIE) Conference Series, pp. 362-375.

Vogt, S. S. \& Penrod, G. D. (1983) Doppler imaging of spotted stars : application to the RS Canum Venaticorum star HR 1099. PASP, 95, 565-576.

Vollmann, K. \& Eversberg, T. (2006) Remarks on statistical errors in equivalent widths. Astronomische Nachrichten, 327(9), 862. 
Walkowicz, L. M., Hawley, S. L. \& West, A. A. (2004) The $\chi$ Factor: Determining the Strength of Activity in Low-Mass Dwarfs. PASP, 116(826), 1105-1110.

Walton, S. R., Preminger, D. G. \& Chapman, G. A. (2003) The Contribution of Faculae and Network to Long-Term Changes in the Total Solar Irradiance. ApJ, 590(2), 1088-1094.

Watson, C. L., Henden, A. A. \& Price, A. (2006) The International Variable Star Index (VSX). Society for Astronomical Sciences Annual Symposium, 25, 47.

WebB, D. F. \& Howard, R. A. (1994) The solar cycle variation of coronal mass ejections and the solar wind mass flux. J. Geophys. Res., 99(A3), 4201-4220.

Webb, D. F. \& Howard, T. A. (2012) Coronal Mass Ejections: Observations. Living Reviews in Solar Physics, 9(1), 3.

Weber, E. J. \& Davis, Leverett, J. (1967) The Angular Momentum of the Solar Wind. ApJ, 148, $217-227$.

Weiss, N. O. (1990) Periodicity and Aperiodicity in Solar Magnetic Activity. Philosophi-cal Transactions of the Royal Society of London Series A, 330(1615), 617-625.

West, A. A., Hawley, S. L., Walkowicz, L. M. et Al. (2004) Spectroscopic Properties of Cool Stars in the Sloan Digital Sky Survey: An Analysis of Magnetic Activity and a Search for Subdwarfs. AJ, 128(1), 426-436.

West, A. A., Weisenburger, K. L., Irwin, J. et Al. (2015) An Activity-Rotation Relationship and Kinematic Analysis of Nearby Mid-to-Late-Type M Dwarfs. ApJ, 812(1), 3.

Wildi, F., Blind, N., Reshetov, V. ET AL. (2017) NIRPS: an adaptive-optics assisted radial velocity spectrograph to chase exoplanets around M-stars. In Proc. SPIE, vol. 10400 of Society of Photo-Optical Instrumentation Engineers (SPIE) Conference Series, pp. 321-335.

Wildi, F., Pepe, F., Chazelas, B., Lo Curto, G. \& Lovis, C. (2010) A Fabry-Perot calibrator of the HARPS radial velocity spectrograph: performance report. In Proc. SPIE, vol. 7735 of Society of Photo-Optical Instrumentation Engineers (SPIE) Conference Series, pp. 1853-1863.

Willson, R. C. \& Hudson, H. S. (1991) The Sun's luminosity over a complete solar cycle. Nature, 351(6321), 42-44.

Wilson, O. C. (1963) A Probable Correlation Between Chromospheric Activity and Age in Main-Sequence Stars. ApJ, 138, 832.

Wilson, O. C. (1968) Flux Measurements at the Centers of Stellar H- and K-Lines. ApJ, 153, 221.

Wilson, O. C. (1978) Chromospheric variations in main-sequence stars. ApJ, 226, 379-396.

WING, R. F. \& FoRd, JR., W. K. (1969) The Infrared Spectrum of the Cool Dwarf Wolf 359. PASP, 81(482), $527-529$.

Wolf, R. (1852) Bericht über neue Untersuchungen über die Periode der Sonnenflecken und ihrer Bedeutung. Astronomische Nachrichten, 35(25), 369.

Wolszczan, A. \& Frail, D. A. (1992) A planetary system around the millisecond pulsar PSR1257 + 12. Nature, 355(6356), 145-147.

Wood, B. E. (2004) Astrospheres and Solar-like Stellar Winds. Living Reviews in Solar Physics, 1(1), 2. 
Wood, B. E. (2006) The Solar Wind and the Sun in the Past. Space Sci. Rev, 126(1-4), 3-14.

Wood, B. E., Müller, H. R., Zank, G. P., Linsky, J. L. \& Redfield, S. (2005) New Mass-Loss Measurements from Astrospheric Ly $\alpha$ Absorption. ApJL, 628(2), L143-L146.

Woolf, V. M. \& Wallerstein, G. (2006) Calibrating M Dwarf Metallicities Using Molecular Indices. PASP, 118(840), 218-226.

Wright, N. J. \& Drake, J. J. (2016) Solar-type dynamo behaviour in fully convective stars without a tachocline. Nature, 535(7613), 526-528.

Wright, N. J., Drake, J. J., Mamajek, E. E. \& Henry, G. W. (2011) The Stellar-activity-Rotation Relationship and the Evolution of Stellar Dynamos. ApJ, 743(1), 48.

Wright, N. J., Newton, E. R., Williams, P. K. G., Drake, J. J. \& Yadav, R. K. (2018) The stellar rotationactivity relationship in fully convective M dwarfs. MNRAS, 479(2), 2351-2360.

Yan, F., Casasayas-Barris, N., Molaverdikhani, K. et al. (2019) Ionized calcium in the atmospheres of two ultra-hot exoplanets WASP-33b and KELT-9b. A\&A, 632, A69.

YANG, H., Liu, J., Gao, Q. ET AL. (2017) The Flaring Activity of M Dwarfs in the Kepler Field. ApJ, 849(1), 36.

Yashiro, S., Akiyama, S., Gopalswamy, N. \& Howard, R. A. (2006) Different Power-Law Indices in the Frequency Distributions of Flares with and without Coronal Mass Ejections. ApJL, 650(2), L143-L146.

Yashiro, S., Gopalswamy, N., Akiyama, S., Michalek, G. \& Howard, R. A. (2005) Visibility of coronal mass ejections as a function of flare location and intensity. Journal of Geophysical Research (Space Physics), 110(A12), A12S05.

Yau, K. K. C. \& Stephenson, F. R. (1988) A revised catalogue of Far Eastern observations of sunspots (165 $\mathrm{BC}$ to AD 1918). QJRAS, 29, 175-197.

Young, A., Skumanich, A., Stauffer, J. R., Bopp, B. W. \& Harlan, E. (1989) A Study of Excess H alpha Emission in Chromospherically Active M Dwarf Stars. ApJ, 344, 427.

Young, C. A. (1872) Letter to the superintendent of the U. S. Coast Survey, containing a catalogue of bright lines in the spectrum of the solar atmosphere, observed at Sherman, Wyoming territory, USA, during July and August, 1872. American Journal of Science, 4(23), 356-361.

Zechmeister, M., Anglada-Escudé, G. \& Reiners, A. (2014) Flat-relative optimal extraction. A quick and efficient algorithm for stabilised spectrographs. A\&A, 561, A59.

Zechmeister, M., Dreizler, S., Ribas, I. et Al. (2019) The CARMENES search for exoplanets around M dwarfs. Two temperate Earth-mass planet candidates around Teegarden's Star. A\&A, 627, A49.

Zechmeister, M. \& Kürster, M. (2009) The generalised Lomb-Scargle periodogram. A new formalism for the floating-mean and Keplerian periodograms. A\&A, 496(2), 577-584.

Zechmeister, M., Kürster, M. \& EndL, M. (2009) The M dwarf planet search programme at the ESO VLT + UVES. A search for terrestrial planets in the habitable zone of M dwarfs. A $\mathcal{E} A, \mathbf{5 0 5}(2), 859-871$.

Zechmeister, M., Reiners, A., Amado, P. J. et al. (2018) Spectrum radial velocity analyser (SERVAL). High-precision radial velocities and two alternative spectral indicators. A\&A, 609, A12.

Zendejas, J., Segura, A. \& Raga, A. C. (2010) Atmospheric mass loss by stellar wind from planets around main sequence M stars. Icarus, 210(2), 539-544.

ZIRIN, H. (1982) $\lambda 10830$ He I observations of 455 stars. ApJ, 260, 655-669. 


\section{Acknowledgements}

The asses of experience go slowly, but even a slow ass reaches its goal eventually. Finishing this thesis would not have been possible without the help and support from many people.

First of all, I would like to thank Ansgar for his support in supervising my $\mathrm{PhD}$ work and many useful discussions. I would also like to thank the other members of my thesis advisory committee, Sandra and Stefan, for the helpful discussions in my TAC meetings. Sandra also helped me a lot with writing papers and this thesis.

I am grateful to everyone in the working group, in particular to Erik, Lev, Mathias, and Oliver, for their ideas and comments, and fruitful discussions on the interpretation of the results. I enjoyed working with the CARMENES consortium and would like to extend thanks in particular to José for many useful comments and discussions.

Finally, thanks to my parents for their enduring support throughout the years, and a happy hee-haw to my friends at SETI.Germany. 